Supporting Information for

\title{
17-Nor-Cephalotane-Type Diterpenoids from Cephalotaxus fortunei
}

Zhan-Peng Ge,$^{\dagger}{ }^{\dagger}$ Hong-Chun Liu, ${ }^{\dagger}$ Guo-Cai Wang, ${ }^{\dagger}$ Qun-Fang Liu, ${ }^{\dagger}$ Cheng-Hui Xu, ${ }^{\dagger}$ Jian Ding, ${ }^{\dagger}$ Yao-Yue Fan, ${ }^{*}, \dagger$ and Jian-Min Yue ${ }^{*, \dagger,}$

${ }^{\dagger}$ State Key Laboratory of Drug Research, Shanghai Institute of Materia Medica, Chinese Academy of Sciences, 555 Zuchongzhi Road, Shanghai 201203, People’s Republic of China

Hniversity of Chinese Academy of Sciences, No. 19A Yuquan Road, Beijing 100049, People's Republic of China

* Corresponding authors

Tel.: +86 21 50806718. Email: jmyue@simm.ac.cn; s040500290@126.com. 


\section{List of Supporting Information}

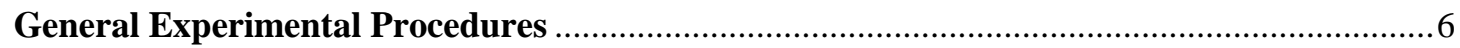

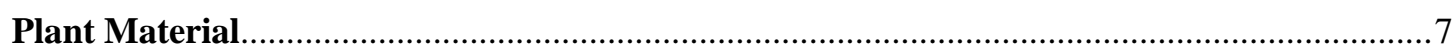

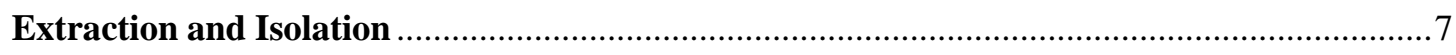

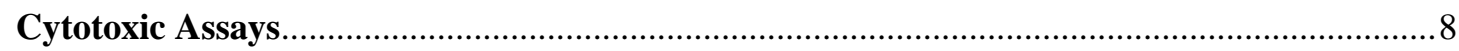

Supplementary Physical and Spectroscopic Data for Compounds 18-20, 22, 24, Cephinoid P,

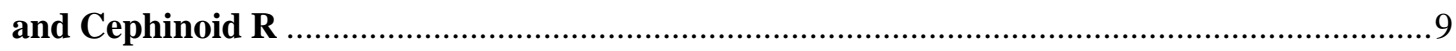

X-ray Crystallographic Analysis for Compounds 1, 5, 13, 18, and 24..................................11

Table S1. Comparison of NMR Data of Our 18 and Du's Fortunolide A .......................................12

Table S2. Comparison of NMR Data of Our 24, Zhao's 14-epi-Cephafortoid A, and Ni's

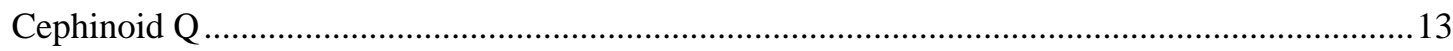

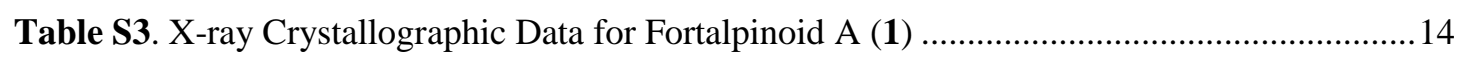

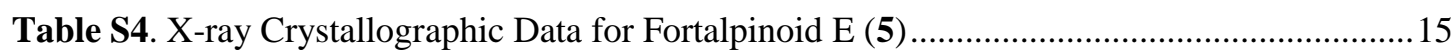

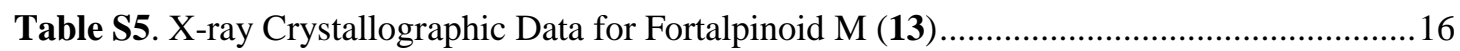

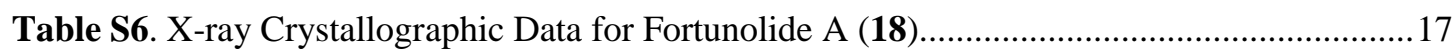

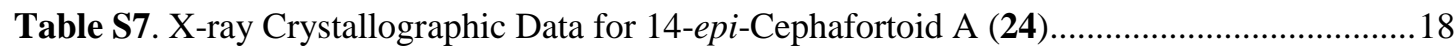

Table S8. Cytotoxicity of Cephalotaxus Troponoids against Tumor Cell Lines............................19

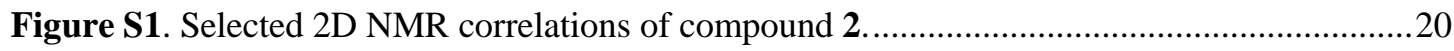

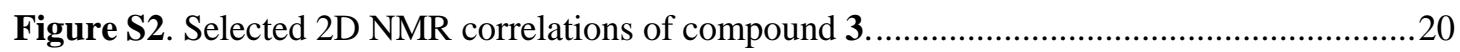

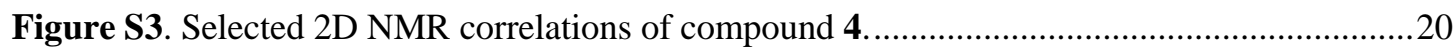

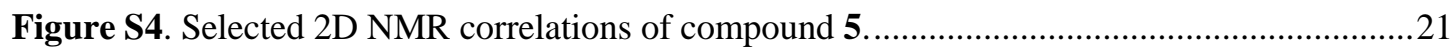

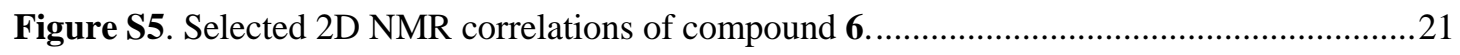

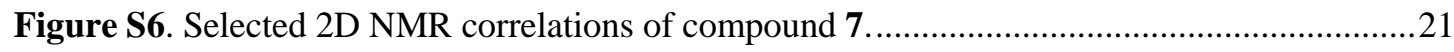

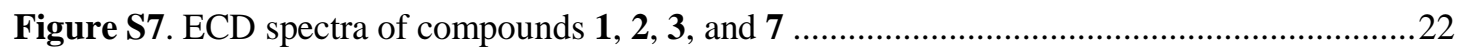

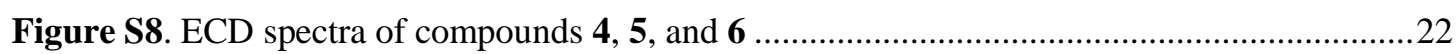

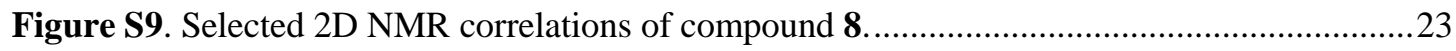

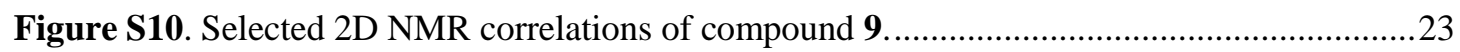

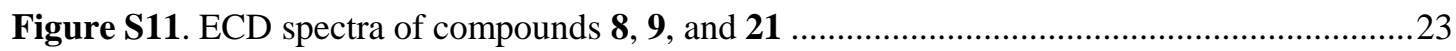

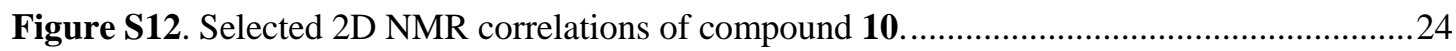

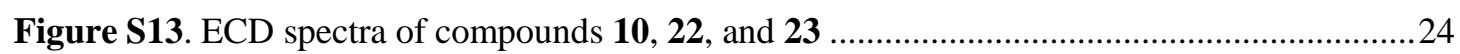

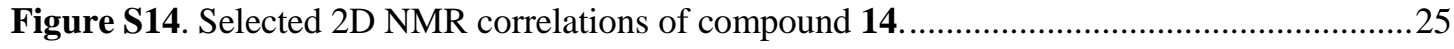

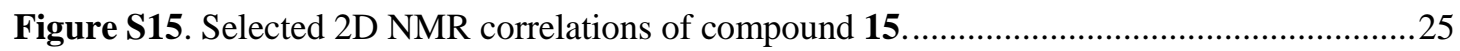

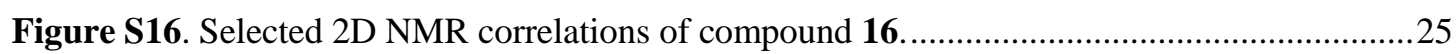

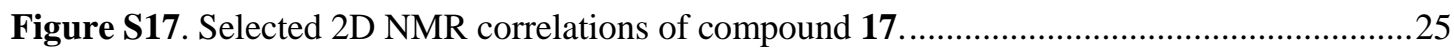

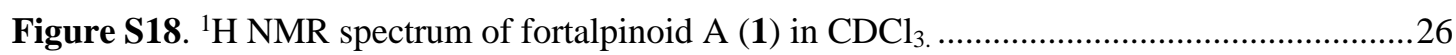

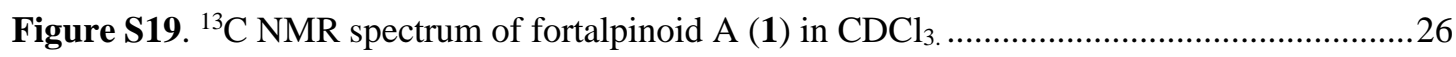

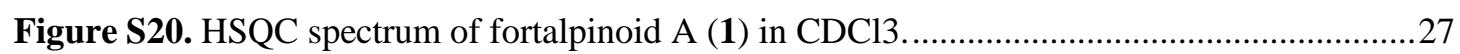

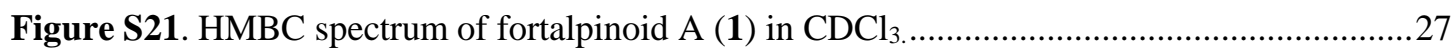

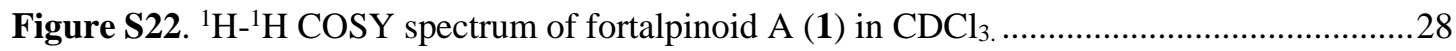




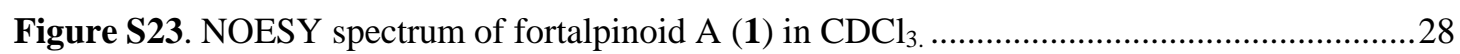

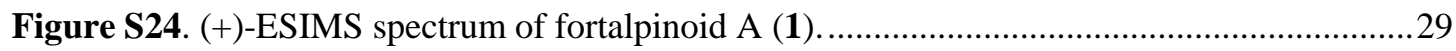

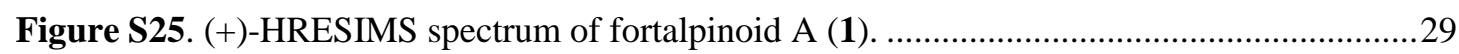

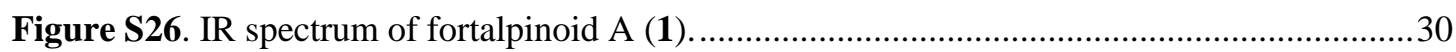

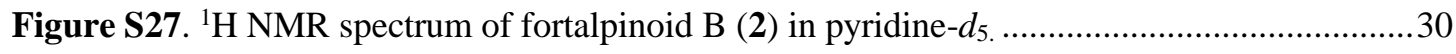

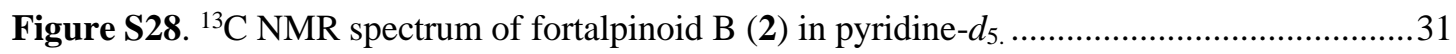

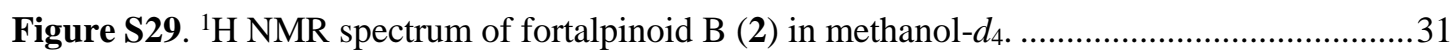

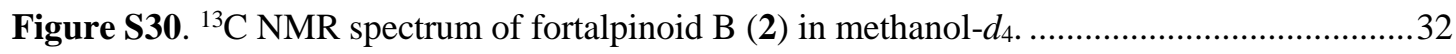

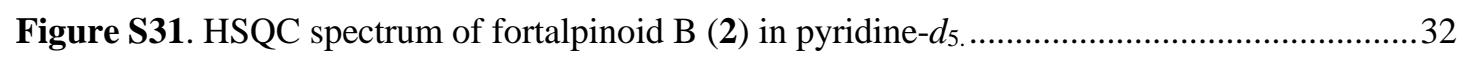

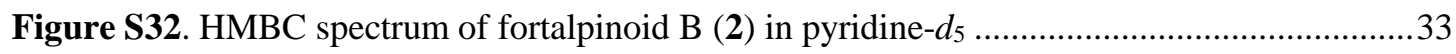

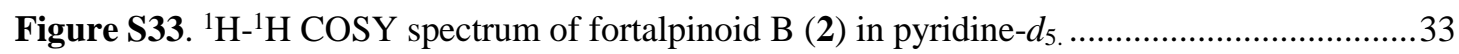

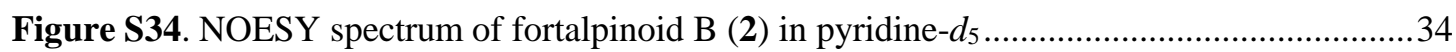

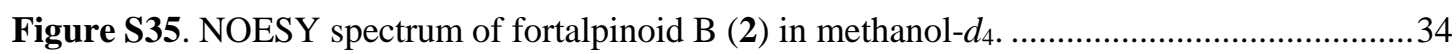

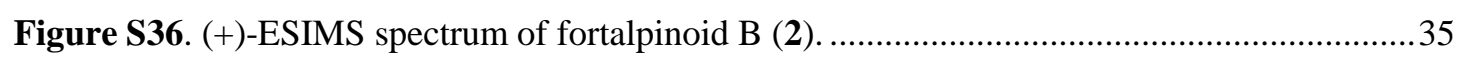

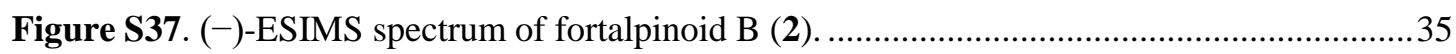

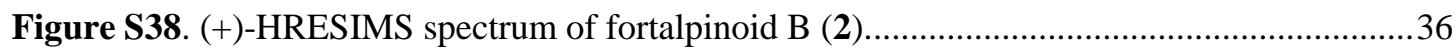

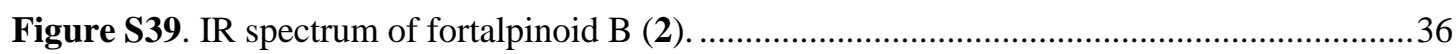

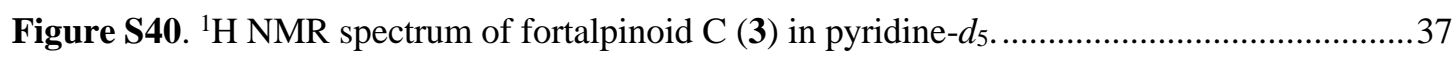

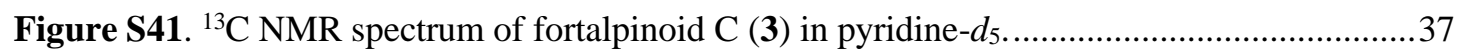

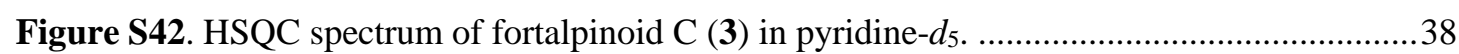

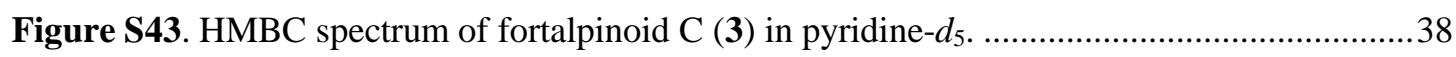

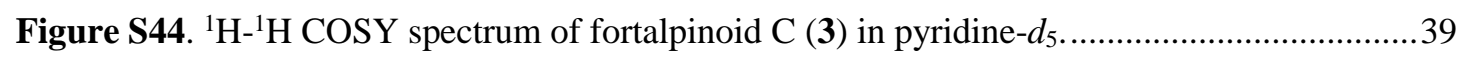

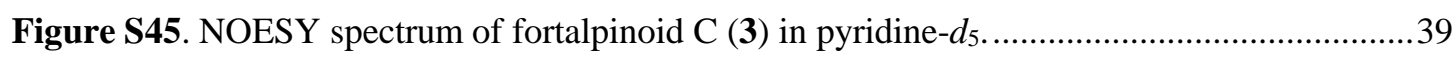

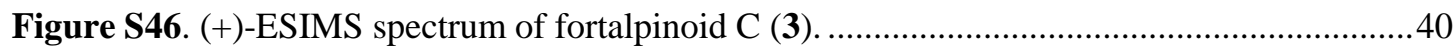

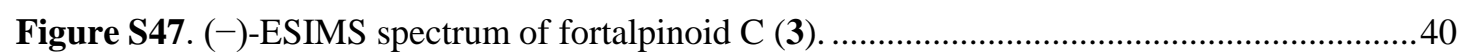

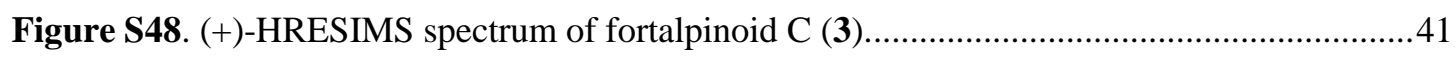

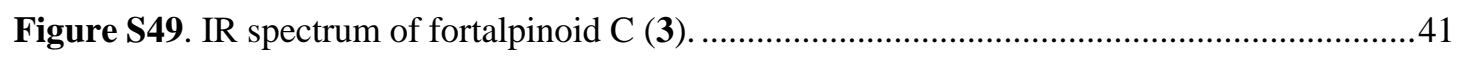

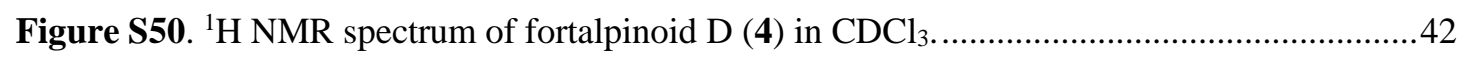

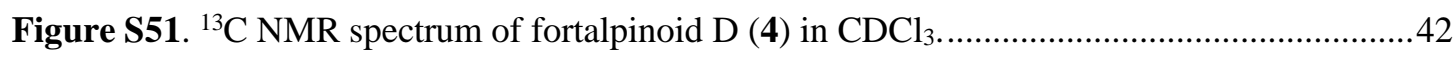

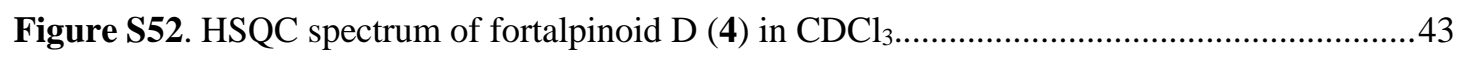

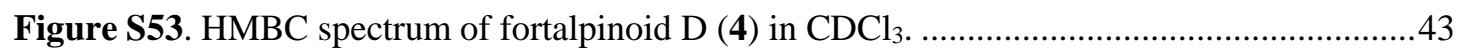

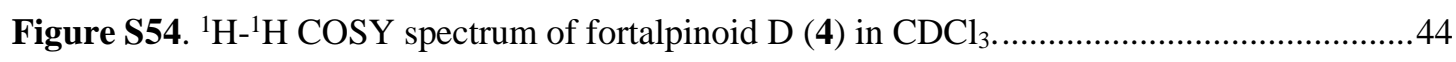

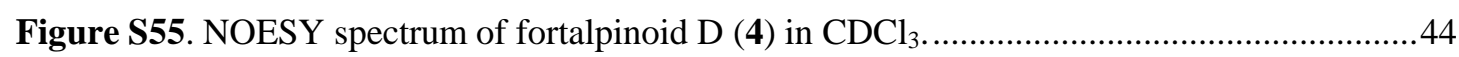

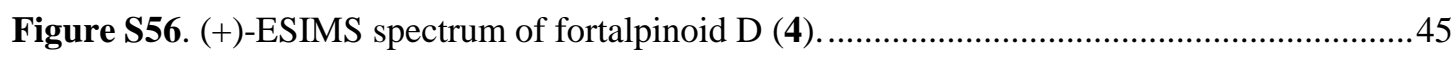

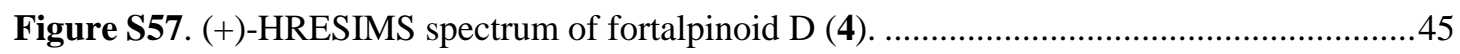

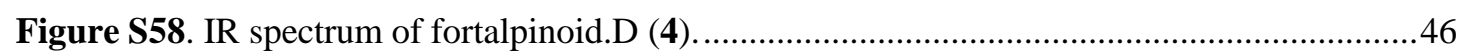

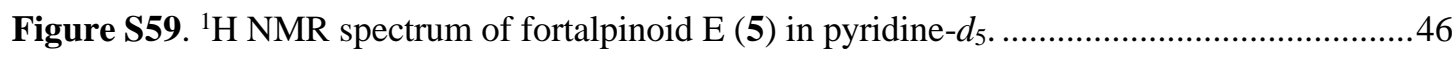

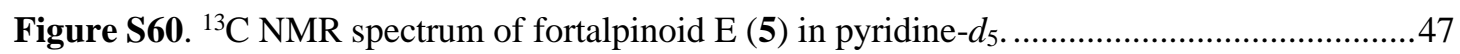

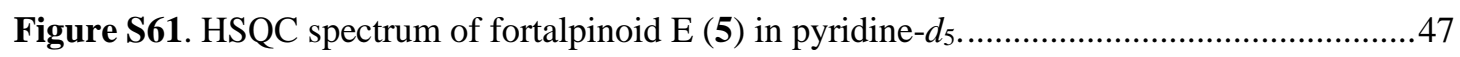

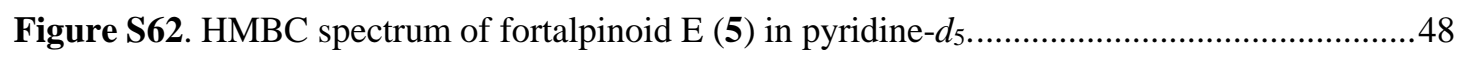

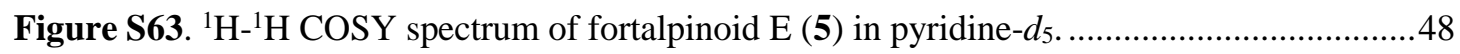

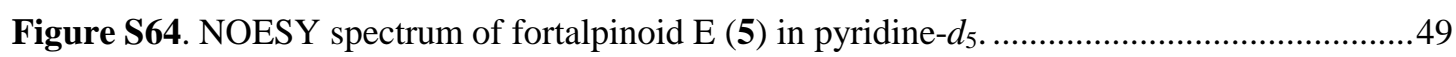

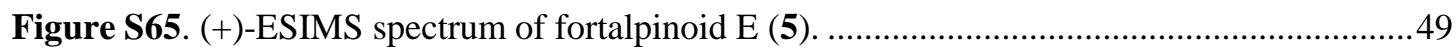

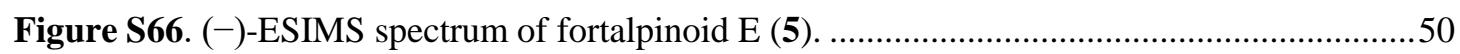




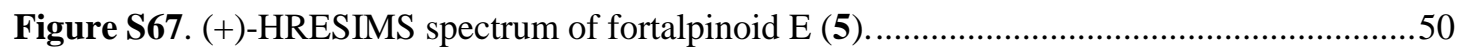

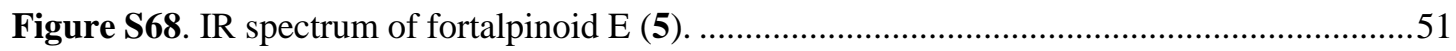

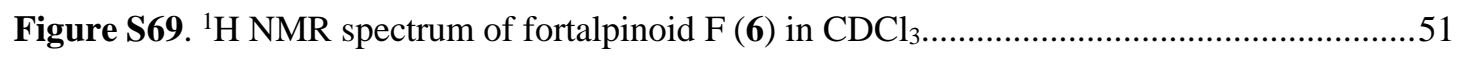

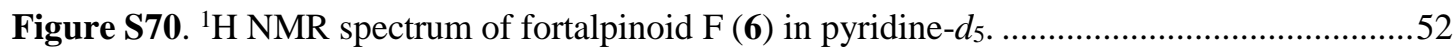

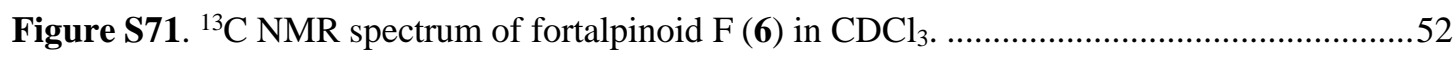

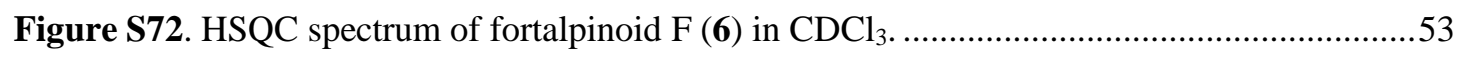

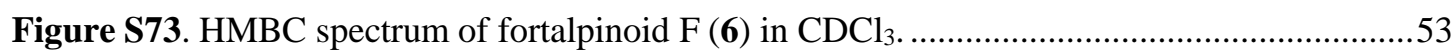

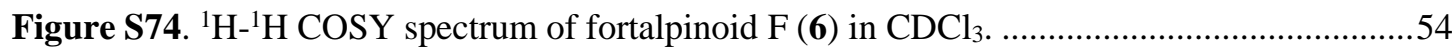

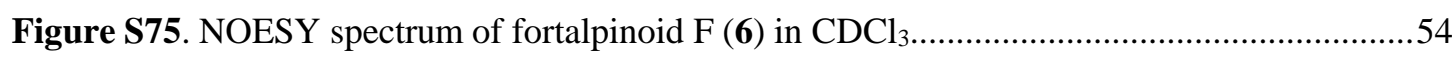

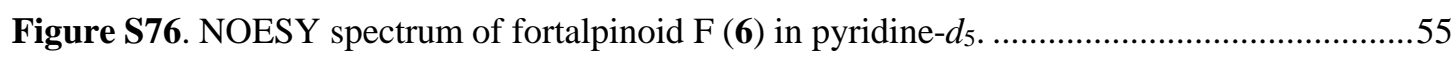

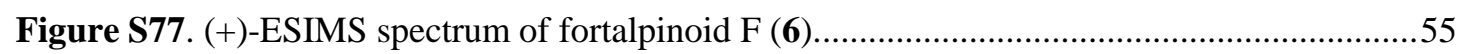

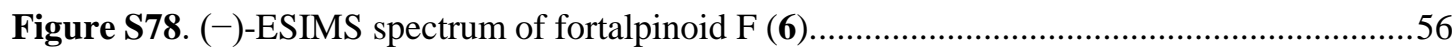

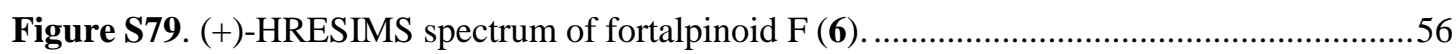

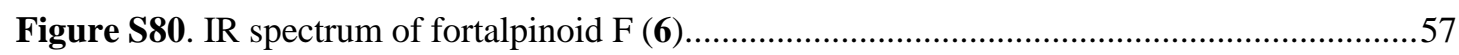

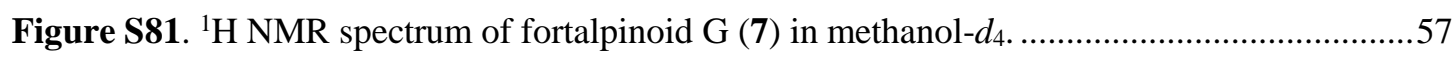

Figure S82. ${ }^{13} \mathrm{C}$ NMR spectrum of fortalpinoid G (7) in methanol- $d_{4} \ldots \ldots \ldots \ldots \ldots \ldots \ldots \ldots \ldots \ldots \ldots \ldots \ldots . . . .58$

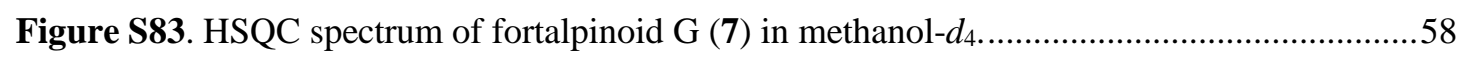

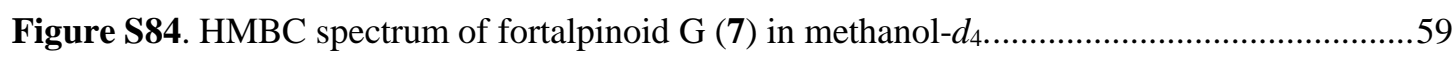

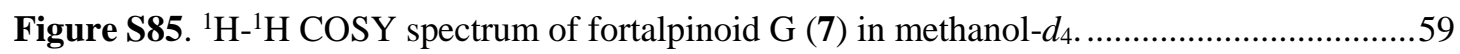

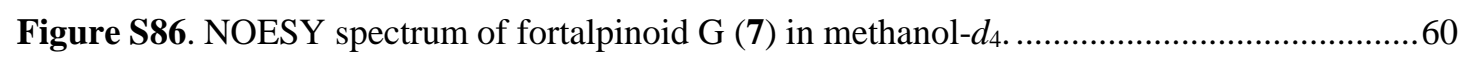

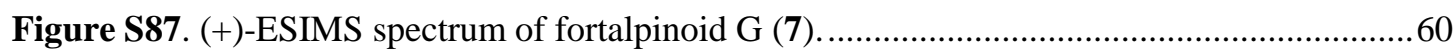

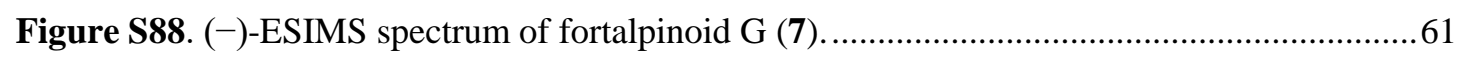

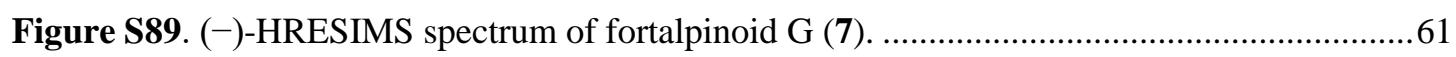

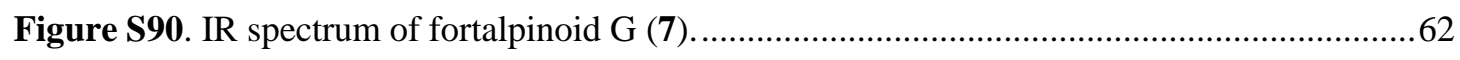

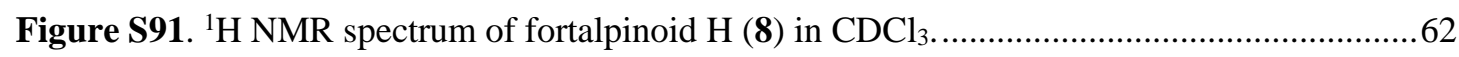

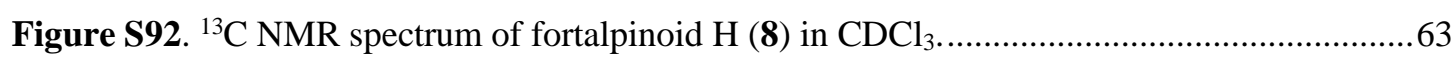

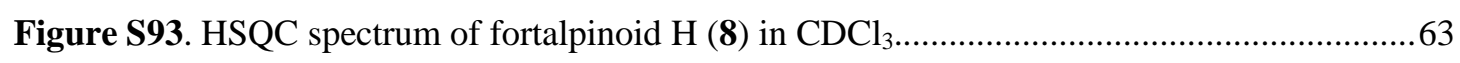

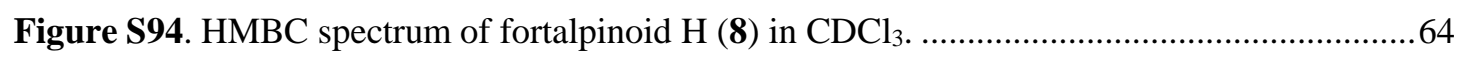

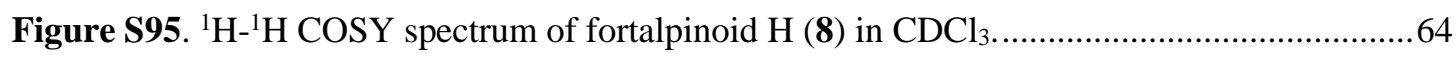

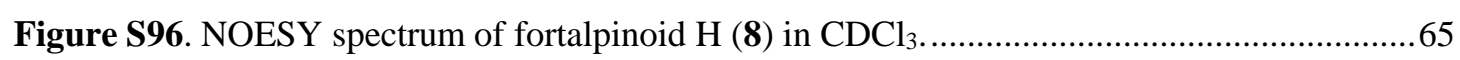

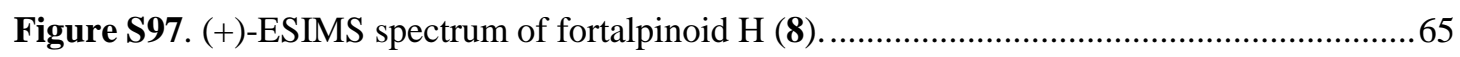

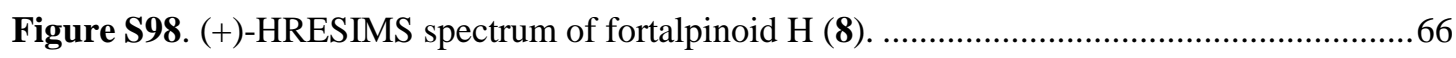

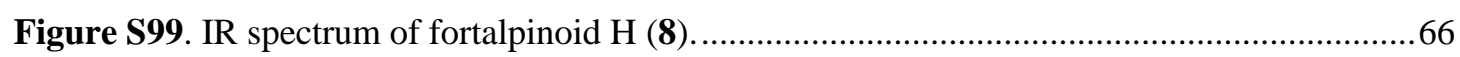

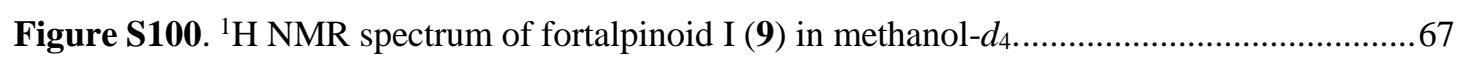

Figure S101. ${ }^{13} \mathrm{C}$ NMR spectrum of fortalpinoid I (9) in methanol- $d_{4} \ldots \ldots \ldots \ldots \ldots \ldots \ldots \ldots \ldots \ldots \ldots \ldots . . . .67$

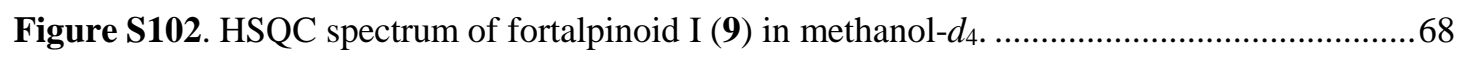

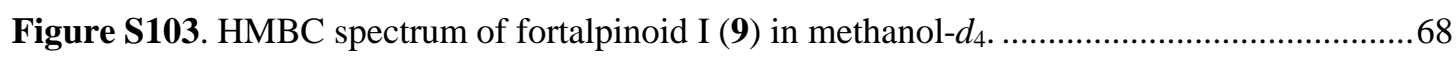

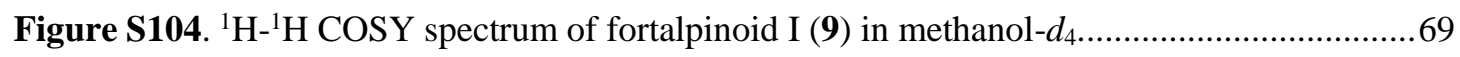

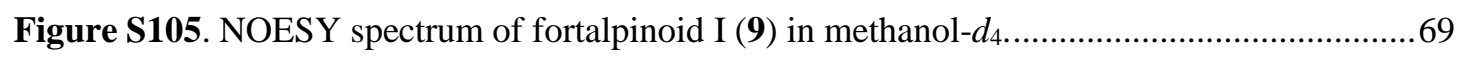

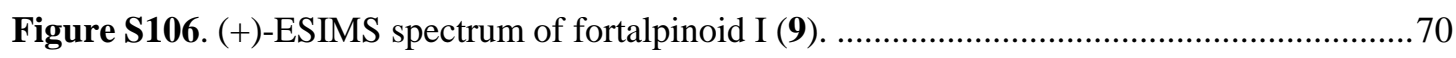

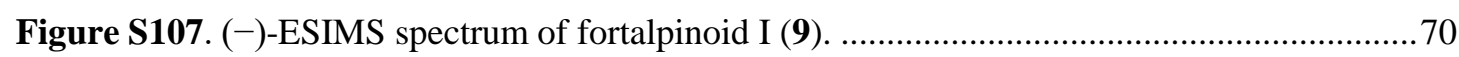

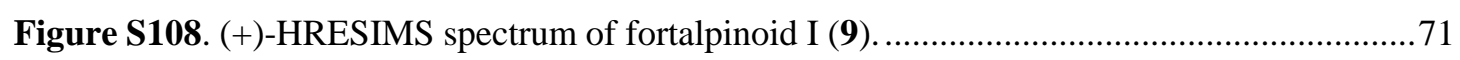

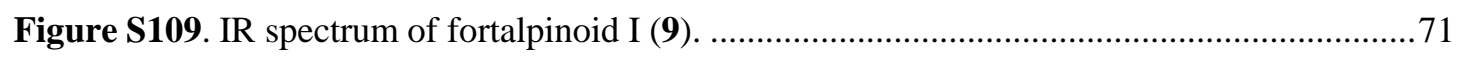

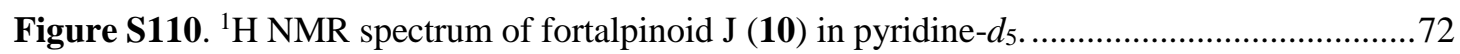




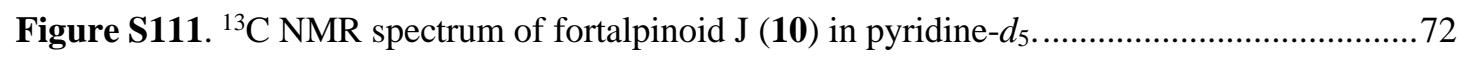

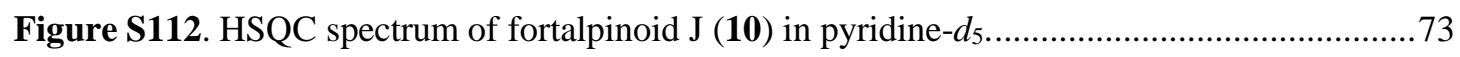

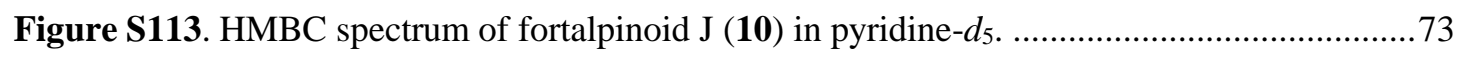

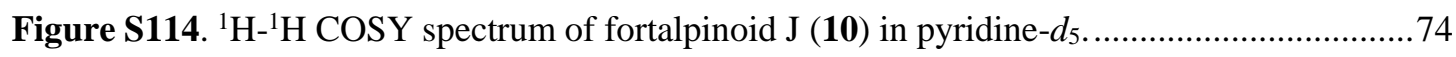

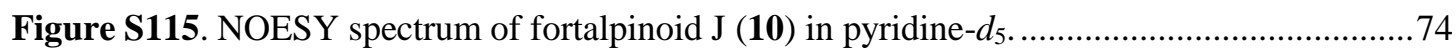

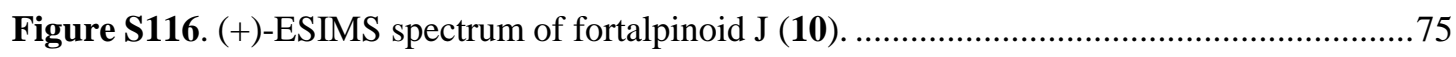

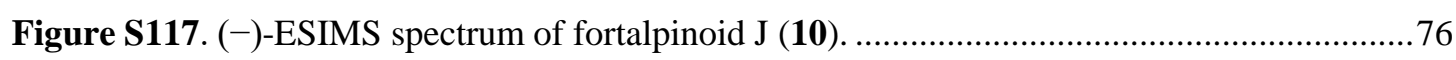

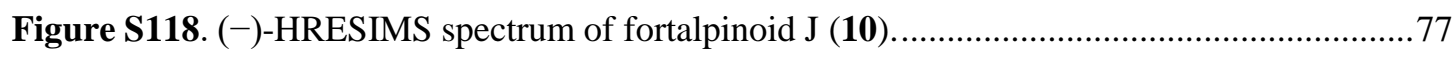

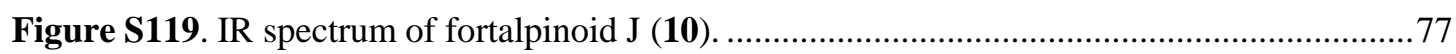

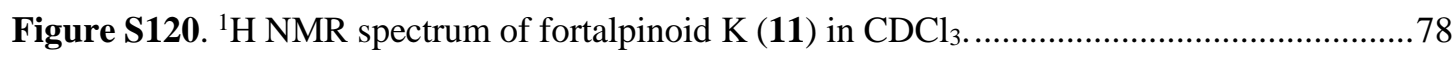

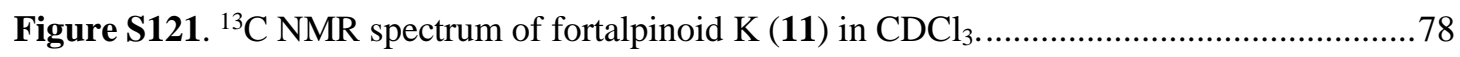

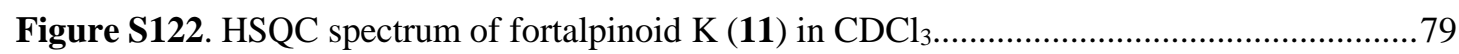

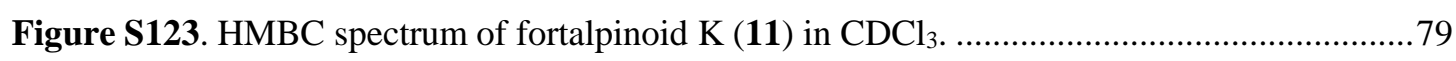

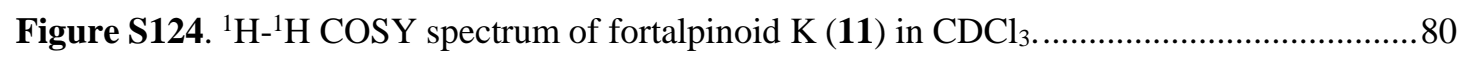

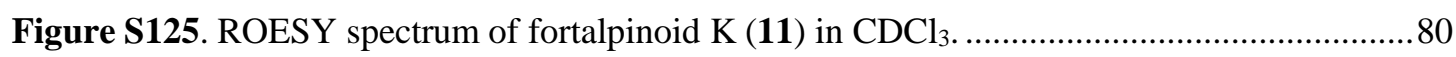

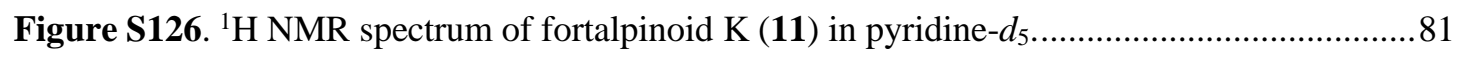

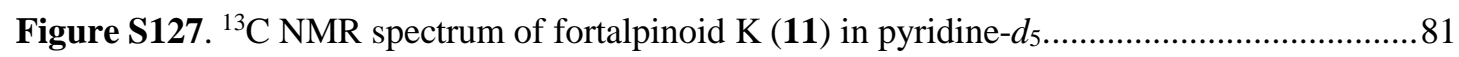

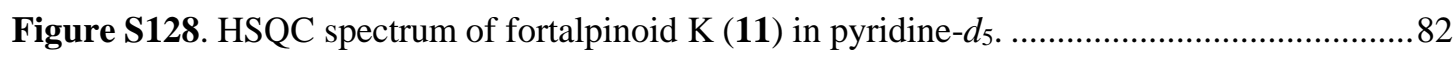

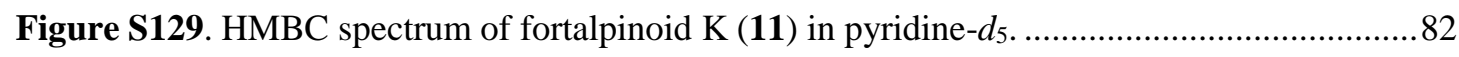

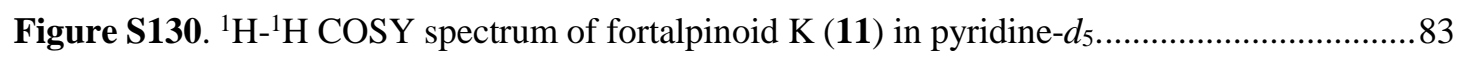

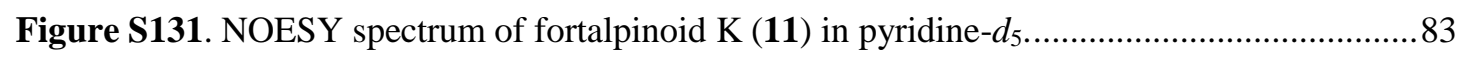

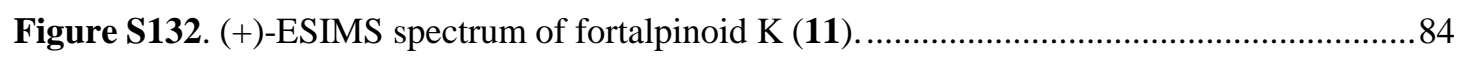

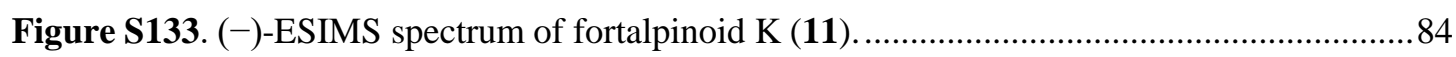

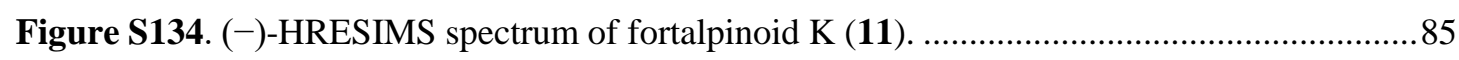

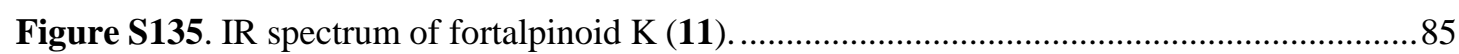

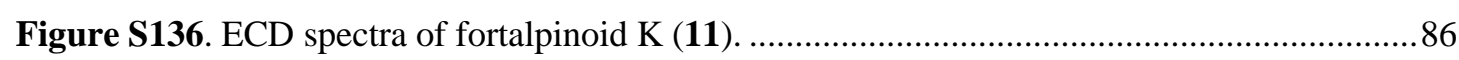

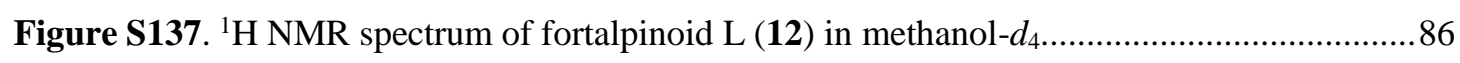

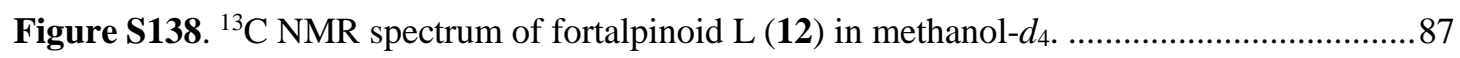

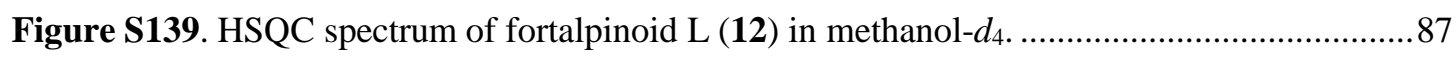

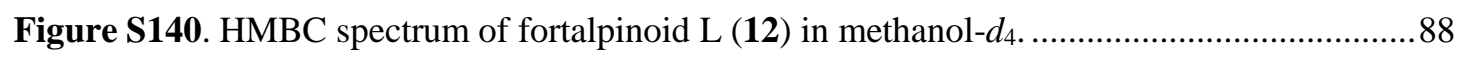

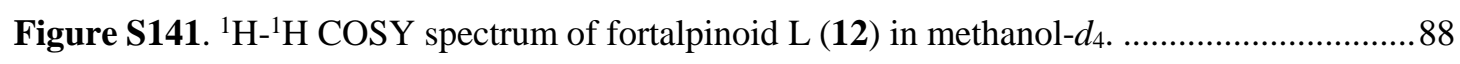

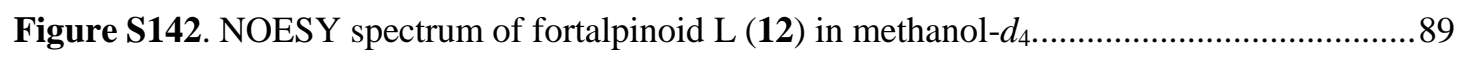

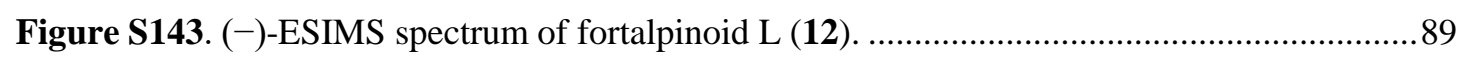

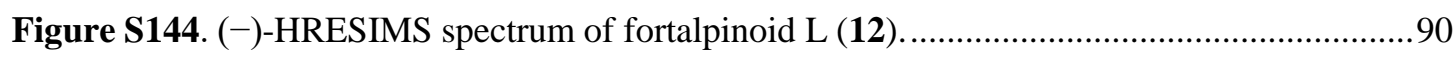

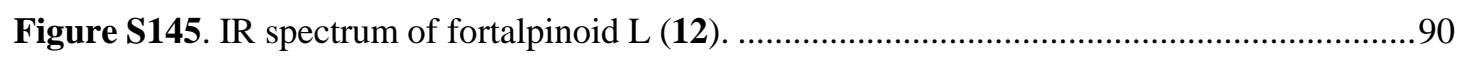

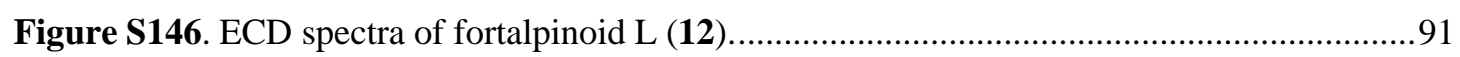

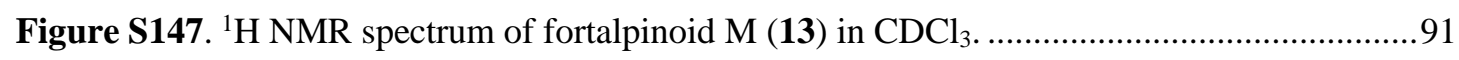

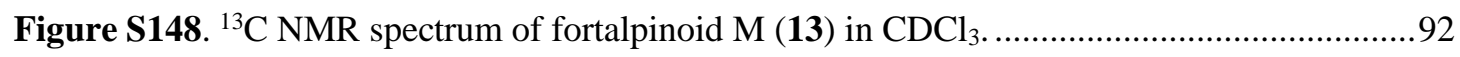

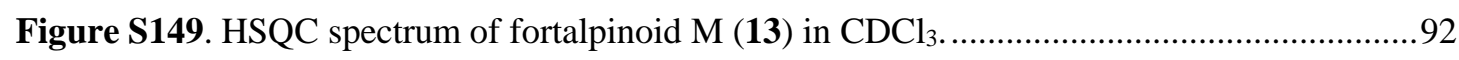

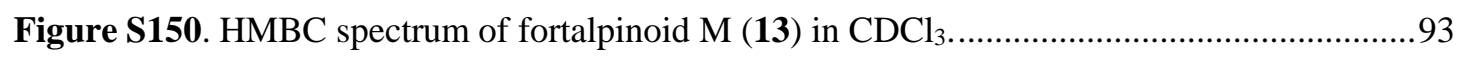

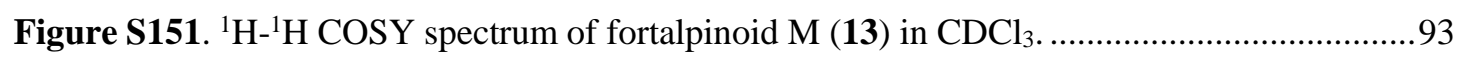

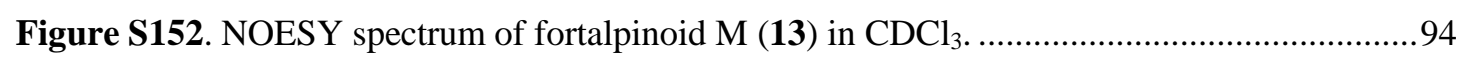

Figure S153. (+)-ESIMS spectrum of fortalpinoid M (13) . .........................................................

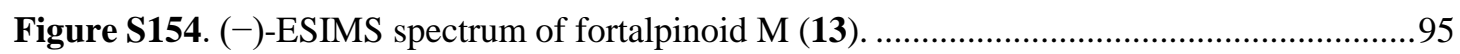




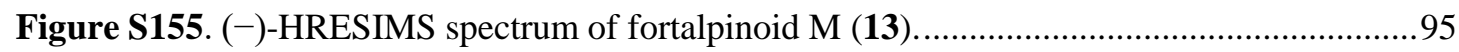

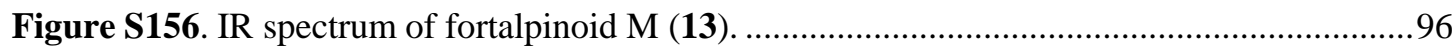

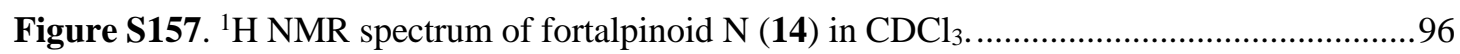

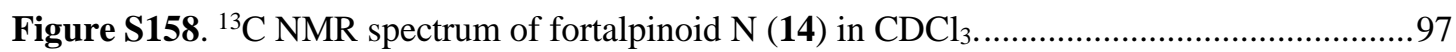

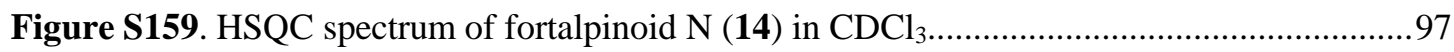

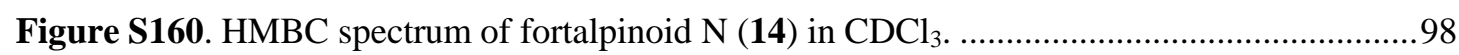

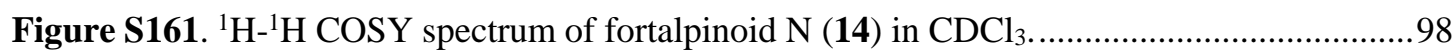

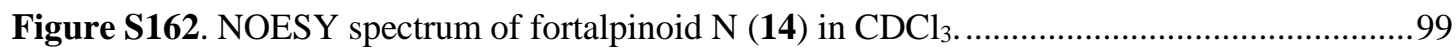

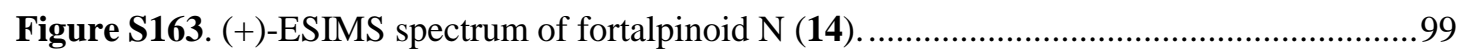

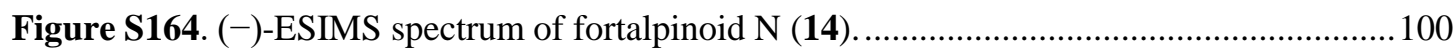

Figure S165. (-)-HRESIMS spectrum of fortalpinoid N (14), ................................................ 100

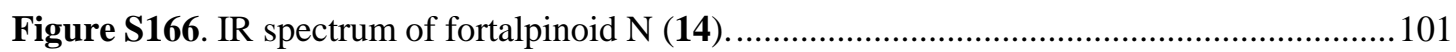

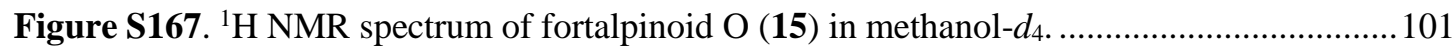

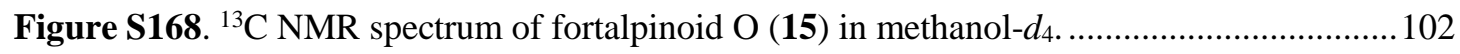

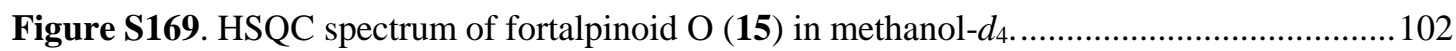

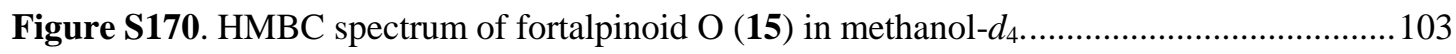

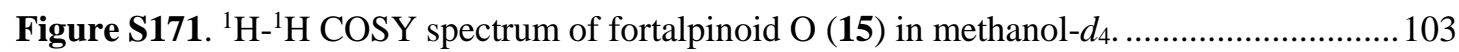

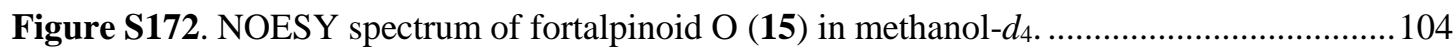

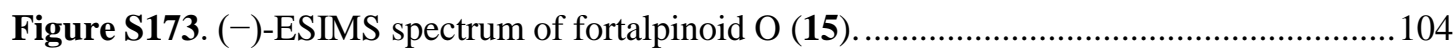

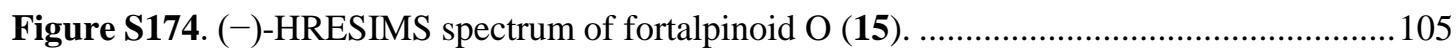

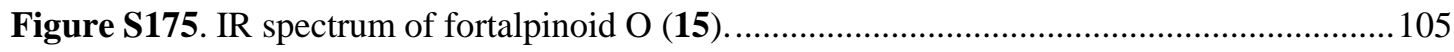

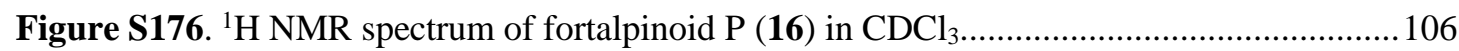

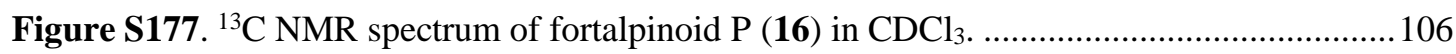

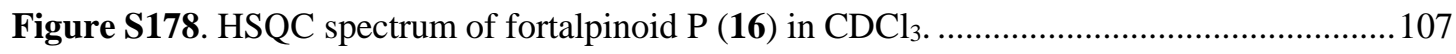

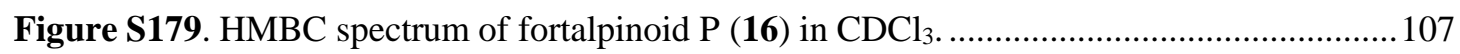

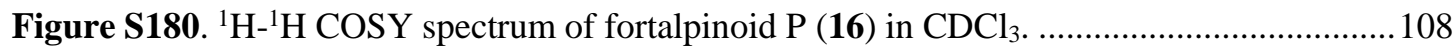

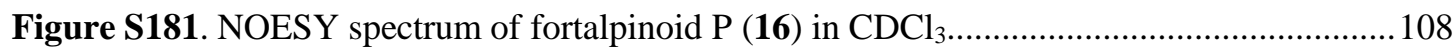

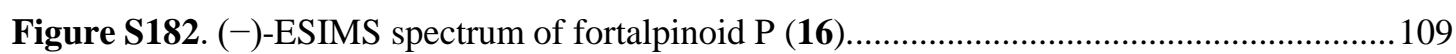

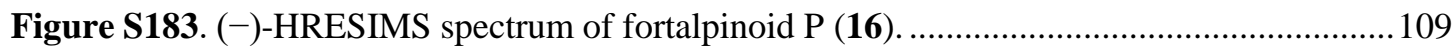

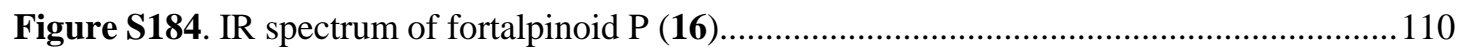

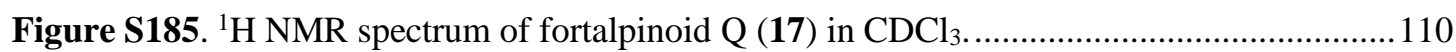

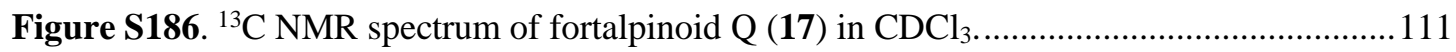

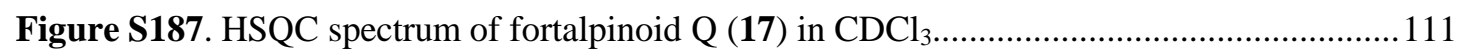

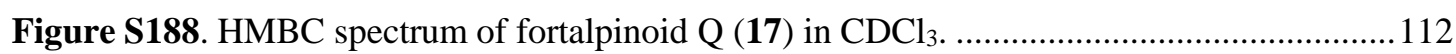

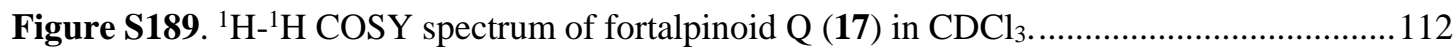

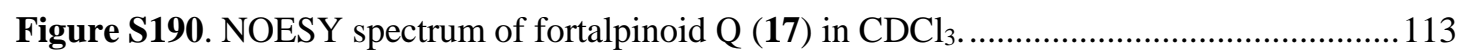

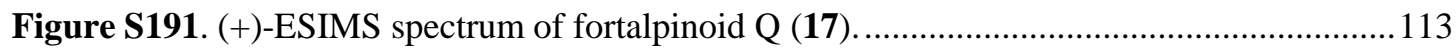

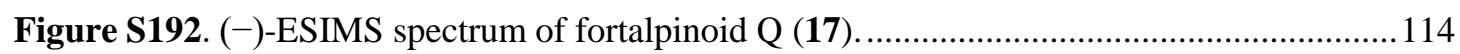

Figure S193. (-)-HRESIMS spectrum of fortalpinoid Q (17). ............................................... 114

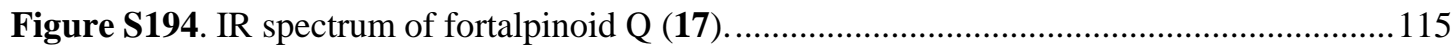




\section{General Experimental Procedures}

Melting points were measured on an SGW X-4 melting point apparatus (Shanghai Precision \& Scientific Instrument Co. Ltd.) and were uncorrected. Optical rotations (Na lamp, $589 \mathrm{~nm}$ ) were obtained with an Autopol VI Automatic Polarimeter at room temperature; concentrations were reported in $\mathrm{g} / 100 \mathrm{~mL}$. UV spectra were recorded on a Shimadzu UV-2550 UV-visible spectrophotometer. ECD spectra were obtained on a JASCO J-810 spectrometer using a $0.05 \mathrm{~cm}$ path length sample cell. IR spectra were recorded on a Thermo Scientific Nicolet iS 5 FT-IR Spectrometer or on a Thermo Scientific Nicolet 6700 FT-IR Spectrometer. NMR spectra were acquired on Bruker Avance III 400, 500, and/or 600 spectrometers (Bruker Biospin AG, Switzerland) and referenced to deuterated solvent peaks. HRESIMS were run on a Waters LCT Premier XE mass spectrometer or an Agilent G6520 Q-TOF mass spectrometer. The X-ray crystallographic data were obtained on a Bruker APEX-II CCD detector employing a graphite-monochromated $\mathrm{Cu}-\mathrm{K} \alpha$ radiation $(1.54 \AA$ ) for compound $\mathbf{1}$, and on a Bruker D8 Venture diffractometer employing Ga-K $\alpha$ radiation (1.34 Å) for compounds 5, 13, 18 and 24. Silica gel (200-300 and 300-400 mesh, Qingdao Haiyang Chemical Co. Ltd., China), MCI gel (CHP20P, 75-150 $\mu \mathrm{m}$, Mitsubishi Chemical Corporation), $\mathrm{C}_{18}$ reversed-phase silica gel (20-45 $\mu \mathrm{m}$, Fuji Silysia Chemical Ltd., Japan), and Sephadex LH-20 (GE Healthcare, Uppsala, Sweden) were used for column chromatography. Semi-preparative HPLC was performed on a Waters 1525 binary pump with a Waters 2489 detector $(254 \mathrm{~nm}$ and $210 \mathrm{~nm})$ using a YMC-Pack ODS-A column $(250 \times 10 \mathrm{~mm}$, S-5 $\mu \mathrm{m}, 12 \mathrm{~nm})$ or a Waters XBridge Prep C 18 column $(250 \times 10 \mathrm{~mm}, \mathrm{~S}-5 \mu \mathrm{m}, 130 \AA$ A $)$. Precoated silica gel $60 \mathrm{~F}_{254}$ plates (Merck Chemicals (Shanghai) Co., Ltd., China) were used for TLC monitors. All solvents were of analytical grade (SCR, Sinopharm Chemical Reagent Co., Ltd., China) except that solvents used for HPLC were of HPLC grade (J\&K Scientific Ltd., China). 


\section{Plant Material}

The seeds of Cephalotaxus fortunei var. alpina (H. L. Li) were collected from Shangri-La, Yunnan province, People's Republic of China, and authenticated by Prof. You-Kai Xu of Xishuangbanna Tropical Botanical Garden, Chinese Academy of Sciences. A voucher specimen has been deposited in Shanghai Institute of Materia Medica, Chinese Academy of Sciences (accession number: Cfsd-2011-1Y-XY).

\section{Extraction and Isolation}

The air-dried and finely ground seeds of $C$. fortunei var. alpina (10 kg) were extracted by maceration in $\mathrm{EtOH} / \mathrm{H}_{2} \mathrm{O}(95: 5,3 \times 20 \mathrm{~L})$ at room temperature giving a crude extract $(1.2 \mathrm{~kg})$. The crude extract was suspended in water and then extracted with petroleum ether and EtOAc sequentially to afford an EtOAc-soluble extract (34 g), which was subjected to an MCI gel column eluted with $\mathrm{MeOH} / \mathrm{H}_{2} \mathrm{O}$ mixtures (30:70 to $100 \% \mathrm{MeOH})$ to give seven fractions $(\mathrm{A}-\mathrm{G})$. Fraction $\mathrm{B}(5.7 \mathrm{~g})$ was separated by column chromatography over silica gel using $\mathrm{CHCl}_{3} / \mathrm{MeOH}$ gradient solvent system (100:1 to 5:1) to yield fractions B1-B5. Fraction C (5.4 g), after precipitation of 18 (350 mg), was subjected to separation over a column of silica gel (petroleum ether/acetone, 10:1 to $1: 2)$ to yield fractions $\mathrm{C} 1-\mathrm{C} 6$, and fraction $\mathrm{D}(5.5 \mathrm{~g})$ was treated similarly to afford fractions D1-D4 after removal of the crystals of 23 (3.0 g). The subfraction B3 (1.2 g) was chromatographed over a column of Sephadex LH-20 with $\mathrm{CHCl}_{3} / \mathrm{MeOH}$ (1:1) to yield five fractions, and the first two was purified by HPLC ( $20 \% \mathrm{CH}_{3} \mathrm{CN}$ in $\left.\mathrm{H}_{2} \mathrm{O}, 3 \mathrm{~mL} / \mathrm{min}\right)$ to give 5 (6 mg, $\left.t_{\mathrm{R}} 15.0 \mathrm{~min}\right), 9\left(1 \mathrm{mg}, t_{\mathrm{R}} 20.0 \mathrm{~min}\right)$, $24\left(5 \mathrm{mg}, t_{\mathrm{R}} 11.3 \mathrm{~min}\right)$, and cephafortoid A (8 mg, $\left.t_{\mathrm{R}} 12.7 \mathrm{~min}\right)$. The subfraction B4 (1.1 g) was fractioned on a reversed-phase $\mathrm{C}_{18}$ silica gel column $\left(\mathrm{MeOH} / \mathrm{H}_{2} \mathrm{O}, 20: 80\right.$ to 80:20) to yield fractions B4a-B4g. Fraction B4b was purified by HPLC $\left(15 \% \mathrm{CH}_{3} \mathrm{CN}\right.$ in $\mathrm{H}_{2} \mathrm{O}$ during 0-20 min, and then $15 \%$ to $27 \%$ during $20-30 \mathrm{~min}, 3 \mathrm{~mL} / \mathrm{min}$ ) to give 7 $\left(1 \mathrm{mg}, t_{\mathrm{R}} 22.8 \mathrm{~min}\right), \mathbf{1 0}\left(3 \mathrm{mg}, t_{\mathrm{R}} 9.0 \mathrm{~min}\right), \mathbf{1 2}\left(1 \mathrm{mg}, t_{\mathrm{R}} 19.2 \mathrm{~min}\right), 15\left(2 \mathrm{mg}, t_{\mathrm{R}} 15.4\right.$ $\mathrm{min})$, and cephinoid R (2 mg, $\left.t_{\mathrm{R}} 14.3 \mathrm{~min}\right)$. Fraction C3 (950 mg) was fractioned over 
a silica gel column $\left(\mathrm{CHCl}_{3} / \mathrm{MeOH}, 100: 1\right.$ to $\left.5: 1\right)$ to give fractions $\mathrm{C} 3 \mathrm{a}-\mathrm{C} 3 \mathrm{~g}$. Fraction C3d was purified by $\mathrm{HPLC}\left(30 \% \mathrm{CH}_{3} \mathrm{CN}\right.$ in $\left.\mathrm{H}_{2} \mathrm{O}\right)$ to give $13\left(3 \mathrm{mg}, t_{\mathrm{R}} 12.4 \mathrm{~min}\right), \mathbf{1 4}$ $\left(10 \mathrm{mg}, t_{\mathrm{R}} 13.2 \mathrm{~min}\right), 17\left(2 \mathrm{mg}, t_{\mathrm{R}} 14.8 \mathrm{~min}\right)$, and cephinoid $\mathrm{P}\left(7 \mathrm{mg}, t_{\mathrm{R}} 9.0 \mathrm{~min}\right)$, and fraction C3f afforded $11\left(1 \mathrm{mg}, t_{\mathrm{R}} 18.5 \mathrm{~min}\right)$ after $\mathrm{HPLC}$ purification $\left(20 \% \mathrm{CH}_{3} \mathrm{CN}\right.$ in $\left.\mathrm{H}_{2} \mathrm{O}\right)$. Similar to the fraction C3, fractions $\mathrm{C} 4(1.5 \mathrm{~g})$, and C5 (1.4 g) were also subjected to a silica gel column and then purified by HPLC ( $3 \mathrm{~mL} / \mathrm{min})$ to yield $2\left(4 \mathrm{mg} ; t_{\mathrm{R}} 12.5\right.$ min, $18 \% \mathrm{CH}_{3} \mathrm{CN}$ in $\left.\mathrm{H}_{2} \mathrm{O}\right), 3\left(2 \mathrm{mg} ; t_{\mathrm{R}} 14.5 \mathrm{~min}, 40 \% \mathrm{MeOH}\right.$ in $\left.\mathrm{H}_{2} \mathrm{O}\right), 4\left(28 \mathrm{mg} ; t_{\mathrm{R}}\right.$ $18.7 \mathrm{~min}, 27 \%$ to $43 \% \mathrm{CH}_{3} \mathrm{CN}$ in $\mathrm{H}_{2} \mathrm{O}$ during $\left.30 \mathrm{~min}\right), 6\left(5 \mathrm{mg} ; t_{\mathrm{R}} 14.0 \mathrm{~min}, 23 \%\right.$ $\mathrm{CH}_{3} \mathrm{CN}$ in $\mathrm{H}_{2} \mathrm{O}$ ), 19 (8 mg; $t_{\mathrm{R}} 13.8 \mathrm{~min}, 25 \% \mathrm{CH}_{3} \mathrm{CN}$ in $\mathrm{H}_{2} \mathrm{O}$ ), 20 (4 mg; $t_{\mathrm{R}} 15.2 \mathrm{~min}$, $25 \% \mathrm{CH}_{3} \mathrm{CN}$ in $\mathrm{H}_{2} \mathrm{O}$ ), and 21 ( $3 \mathrm{mg}$; $t_{\mathrm{R}} 12.5 \mathrm{~min}, 25 \% \mathrm{CH}_{3} \mathrm{CN}$ in $\mathrm{H}_{2} \mathrm{O}$ ). Fraction D1 (315mg) was separated over a column of silica gel $\left(\mathrm{CHCl}_{3} / \mathrm{MeOH}, 100: 1\right.$ to $\left.10: 1\right)$ to give five fractions (D1a-D1e). The fractions D1a and D1c were both purified by HPLC (35\% $\mathrm{CH}_{3} \mathrm{CN}$ in $\mathrm{H}_{2} \mathrm{O}, 3 \mathrm{~mL} / \mathrm{min}$ ), yielding 8 (8 mg, $\left.t_{\mathrm{R}} 12.8 \mathrm{~min}\right)$ and 16 (3 mg, $t_{\mathrm{R}} 14.5$ min), respectively. Similar to the fraction D1, fraction D4 (120mg) was further separated over silica gel and purified by HPLC $\left(25 \% \mathrm{CH}_{3} \mathrm{CN}\right.$ in $\left.\mathrm{H}_{2} \mathrm{O}, 3 \mathrm{~mL} / \mathrm{min}\right)$ to give $1\left(13 \mathrm{mg}, t_{\mathrm{R}} 11.3 \mathrm{~min}\right)$ and $22\left(4 \mathrm{mg}, t_{\mathrm{R}} 12.6 \mathrm{~min}\right)$.

\section{Cytotoxic Assays}

The growth inhibition of human tumor cell lines (A549 and HL-60) was determined by CCK-8 (cell counting kit 8, Dojindo Laboratories, Japan) assay performed according to the manufacturer's instructions with adriamycin as the positive control. Briefly, 90 $\mu \mathrm{L}$ of the cells in logarithmic phase were seeded into $96-$ well plates and allowed to adhere overnight. Then, the cells were incubated in triplicate with compounds at various concentrations for $72 \mathrm{~h}$ followed by the addition of $10 \mu \mathrm{L} \mathrm{CCK}-8$ reagent, and kept in the incubator for further $4 \mathrm{~h}$. The OD value of each well was measured at $450 \mathrm{~nm}$ using a SpectraMax 190 microplate reader (Molecular Devices). 


\section{Supplementary Physical and Spectroscopic Data for Compounds 18-20, 22, 24,}

\section{Cephinoid P, and Cephinoid R}

Fortunolide A (18): mp 269-271 ${ }^{\circ} \mathrm{C} ;[\alpha]^{19.5} \mathrm{D}+77\left(c 0.2\right.$ in $\left.\mathrm{CHCl}_{3}\right) ; \mathrm{ECD}(\mathrm{MeOH}) \lambda$ ( $\Delta \varepsilon) 216$ (3.89), 225 (1.64), 236 (3.40), 257 (-0.39), 287 (2.07), 342 (1.52) nm; ${ }^{1} \mathrm{H}$ $\mathrm{NMR}$ and ${ }^{13} \mathrm{C} \mathrm{NMR}$ data $\left(\mathrm{CDCl}_{3}\right.$ and pyridine- $\left.d_{5}\right)$, and comparison with the reported data, ${ }^{1}$ see table $\mathrm{S} 1$.

3-Deoxyfortalpinoid $F(19)$ : pale yellow solid; $[\alpha]^{19.9} \mathrm{D}+14.0,\left(c 0.2, \mathrm{CHCl}_{3}\right)$; ECD $(\mathrm{MeOH}) \lambda(\Delta \varepsilon) 212$ (-0.21), 222 (-1.35), 241 (2.07), 256 (-0.24), 285 (1.43), 337 (1.20) $\mathrm{nm} ;{ }^{1} \mathrm{H}$ NMR, see reported data ${ }^{2} ;{ }^{13} \mathrm{C}$ NMR $\left(\mathrm{CDCl}_{3}, 126 \mathrm{MHz}\right) \delta 186.3(\mathrm{C}-14), 176.1$ (C-17), 145.9, 145.8, 145.3, 144.8, 141.9 (C-13), 139.6 (C-15), 78.2, 77.2, 49.1 (C-10), 45.5 (C-5), 41.0 (C-1), 31.1 (C-3), 30.5 (C7), 28.5 (C-4), 23.8 (C-16), 21.7 (C-6), 19.4 (C-19).

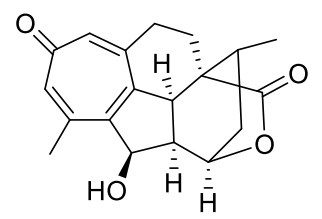

Cephinoid $N$ (20): ${ }^{1} \mathrm{H}$ and ${ }^{13} \mathrm{C}$ NMR data (acetone- $d_{6}$ ) were consistent with reported values ${ }^{3} .{ }^{1} \mathrm{H} \mathrm{NMR}\left(\mathrm{CDCl}_{3}, 400 \mathrm{MHz}\right) \delta 7.39(1 \mathrm{H}, \mathrm{d}, J=2.6 \mathrm{~Hz}), 6.85(1 \mathrm{H}, \mathrm{d}, J=2.6 \mathrm{~Hz})$, $4.82(1 \mathrm{H}, \mathrm{s}), 4.21(1 \mathrm{H}, \mathrm{s}), 3.57(1 \mathrm{H}, \mathrm{d}, J=19.4 \mathrm{~Hz}), 3.39(1 \mathrm{H}, \mathrm{m}), 2.91(1 \mathrm{H}, \mathrm{s}), 2.72(1 \mathrm{H}$, d, $J=19.4 \mathrm{~Hz}), 2.63(1 \mathrm{H}, \mathrm{brd}, J=18.6 \mathrm{~Hz}), 2.25(3 \mathrm{H}, \mathrm{s}), 2.03(1 \mathrm{H}, \mathrm{dd}, J=14.8,10.1 \mathrm{~Hz})$, $1.77(1 \mathrm{H}, \mathrm{m}), 1.56(1 \mathrm{H}, \mathrm{m}), 0.98(3 \mathrm{H}, \mathrm{d}, J=6.8 \mathrm{~Hz}) ;{ }^{13} \mathrm{C} \mathrm{NMR}$ $\left(\mathrm{CDCl}_{3}, 126 \mathrm{MHz}\right) \delta 19.4,24.9,28.8,30.9,35.2,36.1,45.2,50.2$, $77.4,85.9,136.1,140.5,141.9(2 \mathrm{C}), 146.4,147.1,172.1,186.8$, 195.9

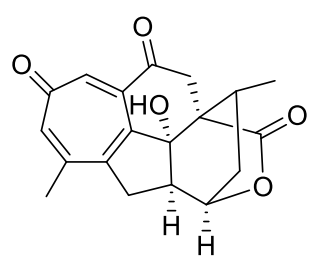

Cephinoid $F(22):[\alpha]{ }^{19.3} \mathrm{D}+80.9,\left(c 0.2, \mathrm{CHCl}_{3}\right) ;{ }^{1} \mathrm{H}$ and ${ }^{13} \mathrm{C}$ NMR data (acetone- $\left.d_{6}\right)$ were consistent with reported values. ${ }^{3}{ }^{1} \mathrm{H} \mathrm{NMR}\left(\mathrm{CDCl}_{3}, 400 \mathrm{MHz}\right) \delta 6.95(1 \mathrm{H}, \mathrm{s}), 6.89$ $(1 \mathrm{H}, \mathrm{d}, J=2.5 \mathrm{~Hz}), 5.35(1 \mathrm{H}, \mathrm{d}, J=5.4 \mathrm{~Hz}), 5.20(1 \mathrm{H}, \mathrm{t}, J=5.7 \mathrm{~Hz}), 4.80(1 \mathrm{H}, \mathrm{d}, J=4.8$ Hz), $4.24(1 \mathrm{H}, \mathrm{d}, J=9.1 \mathrm{~Hz}), 3.98(1 \mathrm{H}, \mathrm{d}, J=5.7 \mathrm{~Hz}), 3.39(1 \mathrm{H}, \mathrm{dt}$, $J=9.1,5.6 \mathrm{~Hz}), 2.91(1 \mathrm{H}, \mathrm{dd}, J=15.9,1.0 \mathrm{~Hz}), 2.38(3 \mathrm{H}, \mathrm{d}, J=1.1$ Hz), $1.74(1 \mathrm{H}, \mathrm{q}, J=7.6 \mathrm{~Hz}), 1.60(1 \mathrm{H}, \mathrm{d}, J=15.9,4.8 \mathrm{~Hz}), 0.89$ $(3 \mathrm{H}, \mathrm{d}, J=7.6 \mathrm{~Hz})$.

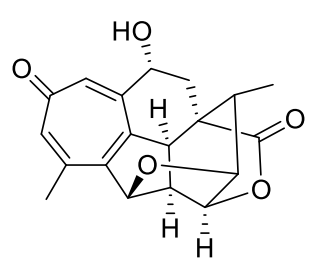

14-epi-Cephafortoid A (24): colorless crystals (MeOH); mp $194-196{ }^{\circ} \mathrm{C} ;[\alpha]^{19.2} \mathrm{D}$ $-108,\left(c 0.8, \mathrm{CHCl}_{3}\right) ;{ }^{1} \mathrm{H} \mathrm{NMR}$ and ${ }^{13} \mathrm{C}$ NMR $\left(\mathrm{CDCl}_{3}\right.$ and acetone- $\left.d_{6}\right)$ data, see table S2. 
Cephinoid P: $[\alpha]^{19.1}{ }_{\mathrm{D}}-294$ (c 0.1, MeOH); CD (MeOH) $\lambda(\Delta \varepsilon) 193$ (-8.57), 202 (-11.1), 240 (8.82), 300 (-4.34) nm; IR (KBr) $\nu_{\max }$ 3957, 2970, 2936, 2877, 1760, 1698, 1640, $1027 \mathrm{~cm}^{-1}$; $1 \mathrm{H} \mathrm{NMR}\left(\mathrm{CDCl}_{3}, 600 \mathrm{MHz}\right) \delta 4.91(1 \mathrm{H}, \mathrm{t}, J=5.4,4.0 \mathrm{~Hz}), 3.78(1 \mathrm{H}$, qd, $J=4.5,4.0,2.9,1.3 \mathrm{~Hz}), 3.04(2 \mathrm{H}, \mathrm{qt}, J=7.4,5.0,3.6 \mathrm{~Hz}), 3.02(1 \mathrm{H}, \mathrm{ddt}, J=7.8,5.1$, $1.2 \mathrm{~Hz}), 2.93(1 \mathrm{H}, \mathrm{dd}, J=13.9,11.9 \mathrm{~Hz}), 2.91(1 \mathrm{H}, \mathrm{dt}, J=7.7,1.4 \mathrm{~Hz}), 2.81(1 \mathrm{H}, \mathrm{tdd}$, $J=11.3,6.3,3.9 \mathrm{~Hz}), 2.73(1 \mathrm{H}, \mathrm{dd}, J=17.6,5.0 \mathrm{~Hz}), 2.65(1 \mathrm{H}, \mathrm{dd}, J=13.9,3.9 \mathrm{~Hz}), 2.64$ $(1 \mathrm{H}, \mathrm{dd}, J=17.5,3.6 \mathrm{~Hz}), 2.63(1 \mathrm{H}, \mathrm{d}, J=2.9 \mathrm{~Hz}), 2.12(1 \mathrm{H}, \mathrm{dt}, J=14.5,4.3,2.5 \mathrm{~Hz})$, $2.08(1 \mathrm{H}$, dddd, $J=14.1,7.0,4.2,2.5 \mathrm{~Hz}), 1.93(1 \mathrm{H}, \mathrm{qd}, J=7.2,4.6 \mathrm{~Hz}), 1.88(1 \mathrm{H}, \mathrm{td}$, $J=14.1,4.3 \mathrm{~Hz}), 1.43$ (1H, tdd, $J=14.0,11.7,4.8 \mathrm{~Hz}), 1.30$ (3H, d, $J=7.4 \mathrm{~Hz}), 1.00$ (3H, $\mathrm{d}, J=7.2 \mathrm{~Hz}) ;{ }^{13} \mathrm{C} \mathrm{NMR}\left(\mathrm{CDCl}_{3}, 126 \mathrm{MHz}\right) \delta 16.1,21.0,24.5,25.6$, 29.8, 34.1, 35.8, 44.8, 46.5, 47.2, 48.6, 48.9, 75.0, 78.4, 146.2, 168.3, 174.6, 203.8, 209.2.

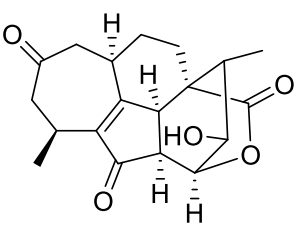

Cephinoid R: $[\alpha]^{19.3}$ D -24.3 (c 0.1, MeOH); CD (MeOH) $\lambda(\Delta \varepsilon) 197$ (3.27), 215 (-3.38), 240 (14.3), 322 (-2.85) nm; IR (KBr) $v_{\max } 3375,2939,2835,1749,1699,1639$, 1025, $738 \mathrm{~cm}^{-1}$; ${ }^{1} \mathrm{H}$ NMR (methanol- $\left.d_{4}, 600 \mathrm{MHz}\right) \delta 4.77(1 \mathrm{H}, \mathrm{t}, J=5.4,3.9 \mathrm{~Hz}$ ), 3.87 $(1 \mathrm{H}, \mathrm{dq}, J=10.5,5.8,4.1 \mathrm{~Hz}), 3.55(1 \mathrm{H}, \mathrm{td}, J=4.8,3.9,1.3 \mathrm{~Hz}), 2.96(1 \mathrm{H}, \mathrm{ttd}, J=11.9$, 11.3, 6.2, 4.3, 0.8 Hz), $2.82(1 \mathrm{H}, \mathrm{hd}, J=7.3,5.8,1.3 \mathrm{~Hz}), 2.70(1 \mathrm{H}, \mathrm{dd}, J=5.5,1.3 \mathrm{~Hz})$, $2.40(1 \mathrm{H}, \mathrm{td}, J=14.1,4.7 \mathrm{~Hz}), 2.23(1 \mathrm{H}, \mathrm{dtd}, J=14.5,5.8,0.6 \mathrm{~Hz}), 2.05(1 \mathrm{H}, \mathrm{dt}, J=13.5$, $4.3 \mathrm{~Hz}), 1.92(1 \mathrm{H}, \mathrm{dtd}, J=13.8,6.4,4.6,2.4 \mathrm{~Hz}), 1.91(1 \mathrm{H}, \mathrm{q}, J=13.6,11.5,10.5 \mathrm{~Hz})$, $1.88(1 \mathrm{H}, \mathrm{p}, J=7.2,4.8 \mathrm{~Hz}), 1.73(1 \mathrm{H}, \mathrm{dt}, J=14.6,5.8 \mathrm{~Hz}), 1.71(1 \mathrm{H}, \mathrm{dt}, J=14.1,4.5,2.3$ Hz), $1.36(1 \mathrm{H}$, tdd, $J=14.0,11.3,4.4 \mathrm{~Hz}), 1.27(3 \mathrm{H}, \mathrm{d}, J=7.3 \mathrm{~Hz}), 0.96$ (3H, d, $J=7.2$ $\mathrm{Hz}) ;{ }^{13} \mathrm{C}$ NMR (methanol- $\left.d_{4}, 126 \mathrm{MHz}\right), 16.1,20.8,22.3,27.4,30.1,34.0,36.2,42.9$, $43.6,52.6,54.3,70.0,75.5,79.6,79.6,146.2,169.9,176.2,203.9$.

Reference

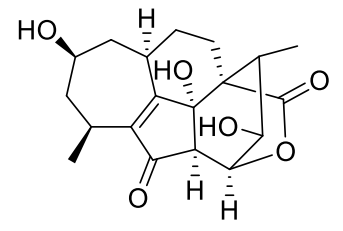

(1) Du, J.; Chiu, M. H.; Nie, R. L., J. Nat. Prod. 1999, 62, 1664-1665.

(2) Rogers, D. H.; Frey, B.; Roden, F. S.; Russkamp, F. W.; Willis, A. C.; Mander, L. N., Aust. J. Chem. 1999, 52, 1093-1108.

(3) Ni, L.; Zhong, X. H.; Chen, X. J.; Zhang, B. J.; Bao, M. F.; Cai, X. H., Phytochemistry. 2018, 151, 50-60. 


\section{X-ray Crystallographic Analysis for Compounds 1, 5, 13, 18, and 24}

The crystal data of compound $\mathbf{1}$ were collected on a Bruker APEX-II CCD detector, employing a graphite-monochromated $\mathrm{Cu}-\mathrm{K} \alpha$ radiation $(\lambda=1.54178 \AA)$ and operating in the $\phi-\omega$ scan mode. The structures were solved by direct methods using SHELXS-97 (Sheldrick 2008), and refinements were performed with SHELXL-2013 (Sheldrick 2013) using full-matrix least-squares calculations on $F^{2}$. All non-hydrogen atoms were refined anisotropically, and the hydrogen atom positions were geometrically idealized and allowed to ride on their parent atoms. The crystal data of compounds 5, 13, 18, and 24 were obtained on a Bruker D8 Venture diffractometer. The crystal was kept at $169.96 \mathrm{~K}$ during data collection. Using Olex $2^{1}$, the structure was solved with the ShelXT ${ }^{2}$ structure solution program using Intrinsic Phasing and refined with the ShelXL ${ }^{3}$ refinement package using Least Squares minimisation.

\section{References}

(1) Dolomanov, O.V., Bourhis, L.J., Gildea, R.J, Howard, J.A.K. \& Puschmann, H. (2009), J. Appl. Cryst. 42, 339-341.

(2) Sheldrick, G.M. (2015). Acta Cryst. A71, 3-8.

(3) Sheldrick, G.M. (2015). Acta Cryst. C71, 3-8. 
Table S1. Comparison of NMR Data of Our 18 and Du's Fortunolide A ${ }^{1}$

\begin{tabular}{|c|c|c|c|c|c|c|}
\hline & \multicolumn{2}{|l|}{18 (pyridine- $d_{5}$ ) } & \multicolumn{2}{|l|}{$18\left(\mathrm{CDCl}_{3}\right)$} & \multicolumn{2}{|c|}{ fortunolide $\mathrm{A}\left(\mathrm{CDCl}_{3}\right)$} \\
\hline No. & $\delta_{\mathrm{H}}(J$ in $\mathrm{Hz})$ & $\delta \mathrm{C}$ & $\delta_{\mathrm{H}}(J$ in $\mathrm{Hz})$ & $\delta \mathrm{C}$ & $\delta_{\mathrm{H}}(J$ in $\mathrm{Hz})$ & $\delta \mathrm{C}$ \\
\hline 1 & $2.99, \mathrm{~m}$ & 46.8 & $2.87, \mathrm{~m}$ & 45.7 & $2.83, \mathrm{~m}$ & 45.6 \\
\hline 2 & $4.79, \mathrm{t}(4.4)$ & 78.3 & $4.74, \mathrm{~m}$ & 77.3 & $4.71, \mathrm{t}(4.4)$ & 77.2 \\
\hline \multirow[t]{2}{*}{3} & 1.37, brd (14.8) & $29.5^{a}$ & 1.45 , overlap & 28.9 & $1.46, \mathrm{~m}$ & 28.8 \\
\hline & $1.87, \mathrm{dd}(14.8,10.2)$ & & 1.85 , overlap & & $1.84, \mathrm{~m}$ & \\
\hline 4 & $1.54, \mathrm{~m}$ & 30.7 & 1.45 , overlap & 30.1 & $1.41, \mathrm{q}(6.8)$ & 30.0 \\
\hline 5 & & 50.9 & & 49.8 & & 49.7 \\
\hline \multirow[t]{2}{*}{6} & 1.99, ddd $(14.2,8.5,4.3)$ & 19.5 & 1.85 , overlap & 17.9 & $1.76, \mathrm{~m}$ & 17.9 \\
\hline & 3.09, ddd $(14.2,9.9,5.6)$ & & $2.69, \mathrm{~m}$ & & $2.65, \mathrm{~m}$ & \\
\hline \multirow[t]{2}{*}{7} & 2.54, ddd $(16.3,8.5,5.6)$ & $29.1^{a}$ & $2.56, \mathrm{~m}$ & 28.7 & $2.52, \mathrm{~m}$ & 28.4 \\
\hline & $3.26, \operatorname{dddd}(16.3,9.9,4.3,1.7)$ & & $3.15, \mathrm{~m}$ & & $3.08, \mathrm{~m}$ & \\
\hline 8 & & $144.5^{b}$ & & 144.4 & & 143.8 \\
\hline 9 & & $144.8^{b}$ & & 146.0 & & 145.1 \\
\hline 10 & & 87.4 & & 86.9 & & 86.8 \\
\hline 11 & & $145.9^{b}$ & & 147.0 & & 146.2 \\
\hline 12 & & $146.5^{b}$ & & 147.8 & & 147.0 \\
\hline 13 & 7.13 , brs & 141.9 & 6.81 , brs & 140.8 & $6.68, \mathrm{~d}(2.0)$ & 140.8 \\
\hline 14 & & 186.3 & & 185.8 & & 186.0 \\
\hline 15 & 7.10 , brs & 140.1 & 6.78 , brs & 138.8 & $6.67, \mathrm{~d}(2.0)$ & 138.8 \\
\hline 16 & $2.02, \mathrm{~s}$ & 24.6 & $2.22, \mathrm{~s}$ & 24.8 & $2.15, \mathrm{~s}$ & 24.4 \\
\hline 18 & & 174.9 & & 173.7 & & 173.5 \\
\hline 19 & $0.94, \mathrm{~d}(7.0)$ & 20.1 & $0.93, \mathrm{~d}(6.1)$ & 19.5 & $0.90, \mathrm{~d}(6.9)$ & 19.3 \\
\hline \multirow[t]{2}{*}{20} & $2.48, \mathrm{dd}(19.0,2.9)$ & 36.2 & 2.48, brd (19.0) & 36.0 & $2.43, \mathrm{dd}(19.0,3.0)$ & 35.7 \\
\hline & $2.90, \mathrm{dd}(19.0,10.0)$ & & $3.34, \mathrm{dd}(19.0,9.0)$ & & $3.28, \mathrm{dd}(19.0,9.5)$ & \\
\hline
\end{tabular}

\section{Reference}

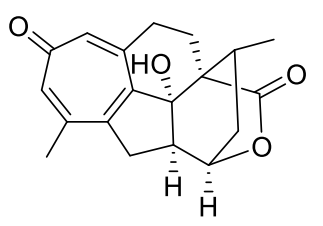

(1) Du, J.; Chiu, M. H.; Nie, R. L., J. Nat. Prod. 1999, 62, 1664-1665. 
Table S2. Comparison of NMR Data of Our 24, Zhao's 14-epi-Cephafortoid A ${ }^{1}$, and Ni’s Cephinoid $Q^{2}$

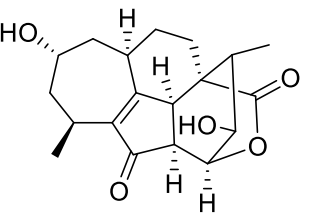

\begin{tabular}{|c|c|c|c|c|c|c|c|c|}
\hline \multirow[b]{2}{*}{ No. } & \multicolumn{2}{|c|}{$\mathbf{2 4}$, in acetone- $d 6$} & \multicolumn{2}{|c|}{$\begin{array}{c}\text { cephinoid } \mathrm{Q}, \text { in } \\
\text { acetone- } d_{6}\end{array}$} & \multicolumn{2}{|r|}{ 24, in $\mathrm{CDCl}_{3}$} & \multicolumn{2}{|c|}{$\begin{array}{c}\text { 14-epi-cephafortoid } \\
\mathrm{A}, \text { in } \mathrm{CDCl}_{3}\end{array}$} \\
\hline & $\delta \mathrm{C}$ & $\begin{array}{c}\delta \mathrm{H} \\
(J \text { in } \mathrm{Hz})\end{array}$ & $\delta \mathrm{C}$ & $\begin{array}{c}\delta \mathrm{H} \\
(J \text { in } \mathrm{Hz})\end{array}$ & $\delta \mathrm{C}$ & $\begin{array}{c}\delta \mathrm{H} \\
(J \text { in } \mathrm{Hz})\end{array}$ & $\delta \mathrm{C}$ & $\begin{array}{c}\delta \mathrm{H} \\
(J \text { in } \mathrm{Hz})\end{array}$ \\
\hline 1 & 45.6 & $2.86, \mathrm{~m}$ & 45.5 & $\begin{array}{l}2.86, \\
\text { overlap }\end{array}$ & 45.0 & 2.94, dd $(7.7,5.3)$ & 45.0 & $\begin{array}{l}2.96, \mathrm{dd} \\
(7.7,5.3)\end{array}$ \\
\hline 2 & 79.4 & $4.68, \mathrm{~m}$ & 79.4 & $\begin{array}{l}4.68, \text { dd } \\
(4.8,4.2)\end{array}$ & 78.5 & 4.85, dd $(5.3,3.9)$ & 78.5 & $\begin{array}{l}4.85, \text { dd } \\
(5.3,3.9)\end{array}$ \\
\hline 3 & 75.8 & $3.60, \mathrm{q}(4.2)$ & 75.7 & $\begin{array}{l}3.60, \mathrm{q} \\
(4.2)\end{array}$ & 75.1 & $3.71, \mathrm{dd}(4.2,3.9)$ & 75.1 & $\begin{array}{l}3.73, \mathrm{t} \\
(3.9)\end{array}$ \\
\hline 4 & 34.6 & $1.94, \mathrm{~m}$ & 34.6 & $\begin{array}{l}1.96, \\
\text { overlap }\end{array}$ & 34.0 & $1.83, \mathrm{~m}$ & 34.1 & $1.84, \mathrm{~m}$ \\
\hline 5 & 46.9 & & 46.8 & & 46.4 & & 46.4 & \\
\hline \multirow[t]{2}{*}{6} & 26.6 & $1.98, \mathrm{~m}$ & 26.6 & $1.99, \mathrm{~m}$ & 25.7 & $1.85, \mathrm{~m}$ & 25.8 & $\alpha 1.86, \mathrm{~m}$ \\
\hline & & $\begin{array}{l}1.82, \operatorname{td}(13.9, \\
4.4)\end{array}$ & & $\begin{array}{l}1.81, \mathrm{td} \\
(13.9,4.5)\end{array}$ & & $2.06, \mathrm{~m}$ & & $\beta 2.07, \mathrm{~m}$ \\
\hline \multirow[t]{2}{*}{7} & 30.5 & $1.93, \mathrm{~m}$ & 30.5 & $\begin{array}{l}1.95, \\
\text { overlap }\end{array}$ & 29.8 & $1.99, \mathrm{~m}$ & 29.8 & $\alpha 2.00, \mathrm{~m}$ \\
\hline & & $1.45, \mathrm{~m}$ & & $1.46, \mathrm{~m}$ & & $\begin{array}{l}1.37, \text { dddd } \\
(14.4,13.5, \\
10.9,4.5)\end{array}$ & & $\beta 1.39, \mathrm{~m}$ \\
\hline 8 & 34.4 & $2.88, \mathrm{~m}$ & 34.3 & $\begin{array}{l}2.87, \\
\text { overlap }\end{array}$ & 33.6 & $2.84, \mathrm{~m}$ & 33.6 & $2.86, \mathrm{~m}$ \\
\hline 9 & 170.0 & & 170.0 & & 171.1 & & 171.0 & \\
\hline 10 & 48.0 & $2.92, \mathrm{~d}(7.9)$ & 48.0 & $\begin{array}{l}2.94 \\
\text { overlap }\end{array}$ & 47.5 & $2.85, \mathrm{~d}(7.8)$ & 47.5 & $\begin{array}{l}2.86, \mathrm{~d} \\
(7.7)\end{array}$ \\
\hline 11 & 147.4 & & 147.4 & & 147.5 & & 147.5 & \\
\hline 12 & 27.7 & $2.76, \mathrm{~m}$ & 27.7 & $2.75, \mathrm{~m}$ & 26.9 & $2.84, \mathrm{~m}$ & 26.9 & $2.85, \mathrm{~m}$ \\
\hline \multirow[t]{2}{*}{13} & 42.2 & $2.09, \mathrm{~m}$ & 42.1 & $\begin{array}{l}2.09, \\
\text { overlap }\end{array}$ & 41.1 & $\begin{array}{l}1.69 \text {, ddd }(13.7,9.8 \text {, } \\
3.2)\end{array}$ & 41.1 & $\beta 1.71, \mathrm{~m}$ \\
\hline & & $\begin{array}{l}1.70, \text { ddd } \\
(13.5,9.8,3.5)\end{array}$ & & $1.70, \mathrm{~m}$ & & $2.15, \mathrm{~m}$ & & $\alpha 2.16, \mathrm{~m}$ \\
\hline 14 & 66.9 & $4.18, \mathrm{~m}$ & 66.7 & $4.18, \mathrm{~m}$ & 67.3 & $4.26, \mathrm{~m}$ & 67.3 & $4.28, \mathrm{~m}$ \\
\hline \multirow[t]{2}{*}{15} & 43.4 & $2.12, \mathrm{~m}$ & 43.4 & $\begin{array}{l}2.14, \\
\text { overlap }\end{array}$ & 42.0 & $1.90, \mathrm{~m}$ & 42.0 & $\alpha 1.91, \mathrm{~m}$ \\
\hline & & $1.90, \mathrm{~m}$ & & $\begin{array}{l}1.93, \\
\text { overlap }\end{array}$ & & $2.12, \mathrm{~m}$ & & $\beta 2.13, \mathrm{~m}$ \\
\hline 16 & 18.6 & $1.15, \mathrm{~d}(7.4)$ & 18.6 & $\begin{array}{l}1.15, \mathrm{~d} \\
(7.2)\end{array}$ & 18.2 & $1.17, \mathrm{~d}(7.3)$ & 18.3 & $\begin{array}{l}1.19, \mathrm{~d} \\
(7.2)\end{array}$ \\
\hline 18 & 175.4 & & 175.4 & & 175.2 & & 175.1 & \\
\hline 19 & 16.2 & $0.90, \mathrm{~d}(7.1)$ & 16.3 & $\begin{array}{l}0.91, \mathrm{~d} \\
(6.6)\end{array}$ & 16.1 & $0.96, \mathrm{~d}(7.2)$ & 16.1 & $\begin{array}{l}0.98, \mathrm{~d} \\
(7.2)\end{array}$ \\
\hline 20 & 203.4 & & 203.3 & & 204.8 & & 204.6 & \\
\hline $\begin{array}{l}3- \\
\mathrm{OH}\end{array}$ & & 4.47, brs & & $\begin{array}{l}4.52, \mathrm{~d} \\
(4.2)\end{array}$ & & & & \\
\hline $\begin{array}{l}14- \\
\mathrm{OH}\end{array}$ & & 3.68, brs & & $\begin{array}{l}3.72, \mathrm{~d} \\
(4.2)\end{array}$ & & & & \\
\hline
\end{tabular}

\section{Reference}

(1) Zhao, J. X.; Fan, Y. Y.; Xu, J. B.; Gan, L. S.; Xu, C. H.; Ding, J.; Yue, J. M., J. Nat. Prod. 2017, $80,356-362$.

(2) Ni, L.; Zhong, X. H.; Chen, X. J.; Zhang, B. J.; Bao, M. F.; Cai, X. H., Phytochemistry. 2018, 151, 50-60. 
Table S3. X-ray Crystallographic Data for Fortalpinoid A (1) ${ }^{a}$

\begin{tabular}{|c|c|}
\hline Empirical formula & $\mathrm{C}_{19} \mathrm{H}_{20} \mathrm{O}_{4}$ \\
\hline Formula weight & 312.35 \\
\hline Temperature & $296(2) \mathrm{K}$ \\
\hline Wavelength & $1.54178 \AA$ \\
\hline Crystal system & Orthorhombic \\
\hline Space group & P 212121 \\
\hline \multirow[t]{3}{*}{ Unit cell dimensions } & $\mathrm{a}=7.0950(2) \AA, \alpha=90^{\circ}$. \\
\hline & $\mathrm{b}=9.6876(2) \AA, \beta=90^{\circ}$. \\
\hline & $\mathrm{c}=22.3117(5) \AA, \gamma=90^{\circ}$. \\
\hline Volume & $1533.56(6) \AA^{3}$ \\
\hline $\mathrm{Z}$ & 4 \\
\hline Density (calculated) & $1.353 \mathrm{Mg} / \mathrm{m}^{3}$ \\
\hline Absorption coefficient & $0.766 \mathrm{~mm}^{-1}$ \\
\hline $\mathrm{F}(000)$ & 664 \\
\hline Crystal size & $0.200 \times 0.170 \times 0.120 \mathrm{~mm}^{3}$ \\
\hline Theta range for data collection & 6.547 to $66.979^{\circ}$ \\
\hline Index ranges & $-8<=\mathrm{h}<=8,-10<=\mathrm{k}<=11,-26<=1<=24$ \\
\hline Reflections collected & 27589 \\
\hline Independent reflections & $2731[\mathrm{R}(\mathrm{int})=0.0438]$ \\
\hline Completeness to theta $=67.679^{\circ}$ & $97.9 \%$ \\
\hline Absorption correction & Semi-empirical from equivalents \\
\hline Max. and min. transmission & 0.7456 and 0.6462 \\
\hline Refinement method & Full-matrix least-squares on $\mathrm{F}^{2}$ \\
\hline Data / restraints / parameters & $2731 / 0 / 212$ \\
\hline Goodness-of-fit on $\mathrm{F}^{2}$ & 1.052 \\
\hline Final $R$ indices [I>2sigma(I)] & $\mathrm{R} 1=0.0329, \mathrm{wR} 2=0.0892$ \\
\hline $\mathrm{R}$ indices (all data) & $\mathrm{R} 1=0.0345, \mathrm{wR} 2=0.0910$ \\
\hline Absolute structure parameter & $-0.04(6)$ \\
\hline Extinction coefficient & $0.0071(18)$ \\
\hline Largest diff. peak and hole & 0.123 and -0.103 e. $\AA^{-3}$ \\
\hline
\end{tabular}

${ }^{a}$ Crystals of 1 were obtained from methanol. 
Table S4. X-ray Crystallographic Data for Fortalpinoid E (5) ${ }^{a}$

\begin{tabular}{|c|c|}
\hline Empirical formula & $\mathrm{C}_{19} \mathrm{H}_{20} \mathrm{O}_{5}$ \\
\hline Formula weight & 327.34 \\
\hline Temperature & $169.98 \mathrm{~K}$ \\
\hline Wavelength & $1.34139 \AA$ \\
\hline Crystal system & Orthorhombic \\
\hline Space group & $\mathrm{P} 2{ }_{1} 2_{1} 2_{1}$ \\
\hline \multirow[t]{3}{*}{ Unit cell dimensions } & $\mathrm{a}=7.64650(10) \AA, \alpha=90^{\circ}$. \\
\hline & $\mathrm{b}=12.9601(2) \AA, \beta=90^{\circ}$. \\
\hline & $\mathrm{c}=15.2245(3) \AA, \gamma=90^{\circ}$ \\
\hline Volume & $1508.74(4) \AA^{3}$ \\
\hline $\mathrm{Z}$ & 4 \\
\hline Density (calculated) & $1.441 \mathrm{Mg} / \mathrm{m}^{3}$ \\
\hline Absorption coefficient & $0.551 \mathrm{~mm}^{-1}$ \\
\hline $\mathrm{F}(000)$ & 692 \\
\hline Crystal size & $0.1 \times 0.08 \times 0.06 \mathrm{~mm}^{3}$ \\
\hline Theta range for data collection & 3.897 to $54.886^{\circ}$ \\
\hline Index ranges & $-9<=\mathrm{h}<=7,-15<=\mathrm{k}<=15,-18<=1<=18$ \\
\hline Reflections collected & 16118 \\
\hline Independent reflections & $2820[\mathrm{R}(\mathrm{int})=0.0315]$ \\
\hline Completeness to theta $=53.594^{\circ}$ & $97.8 \%$ \\
\hline Absorption correction & Semi-empirical from equivalents \\
\hline Max. and min. transmission & 0.7508 and 0.6294 \\
\hline Refinement method & Full-matrix least-squares on $\mathrm{F}^{2}$ \\
\hline Data / restraints / parameters & $2820 / 0 / 221$ \\
\hline Goodness-of-fit on $\mathrm{F}^{2}$ & 1.083 \\
\hline Final $\mathrm{R}$ indices [I>2sigma(I)] & $\mathrm{R} 1=0.0358, \mathrm{wR} 2=0.1021$ \\
\hline $\mathrm{R}$ indices (all data) & $\mathrm{R} 1=0.0363, \mathrm{wR} 2=0.1028$ \\
\hline Absolute structure parameter & $0.01(5)$ \\
\hline Extinction coefficient & $\mathrm{n} / \mathrm{a}$ \\
\hline Largest diff. peak and hole & 0.468 and -0.394 e. $\AA^{-3}$ \\
\hline
\end{tabular}

${ }^{a}$ Crystals of $\mathbf{5}$ were obtained from methanol. 
Table S5. X-ray Crystallographic Data for Fortalpinoid M (13 $)^{a}$

\begin{tabular}{|c|c|}
\hline Empirical formula & $\mathrm{C}_{19} \mathrm{H}_{24} \mathrm{O}_{4}$ \\
\hline Formula weight & 316.38 \\
\hline Temperature & $169.98 \mathrm{~K}$ \\
\hline Wavelength & $1.34139 \AA$ \\
\hline Crystal system & Orthorhombic \\
\hline Space group & $\mathrm{P} 2_{1} 2_{1} 2_{1}$ \\
\hline \multirow[t]{3}{*}{ Unit cell dimensions } & $\mathrm{a}=9.0757(2) \AA, \alpha=90^{\circ}$. \\
\hline & $\mathrm{b}=10.2698(2) \AA, \beta=90^{\circ}$. \\
\hline & $c=16.8446(3) \AA, \gamma=90^{\circ}$. \\
\hline Volume & $1570.01(5) \AA^{3}$ \\
\hline $\mathrm{Z}$ & 4 \\
\hline Density (calculated) & $1.338 \mathrm{Mg} / \mathrm{m}^{3}$ \\
\hline Absorption coefficient & $0.481 \mathrm{~mm}^{-1}$ \\
\hline $\mathrm{F}(000)$ & 680 \\
\hline Crystal size & $0.15 \times 0.12 \times 0.08 \mathrm{~mm}^{3}$ \\
\hline Theta range for data collection & 4.387 to $54.904^{\circ}$ \\
\hline Index ranges & $-11<=\mathrm{h}<=8,-12<=\mathrm{k}<=12,-20<=\mathrm{l}<=16$ \\
\hline Reflections collected & 12577 \\
\hline Independent reflections & $2950[\mathrm{R}(\mathrm{int})=0.0446]$ \\
\hline Completeness to theta $=53.594^{\circ}$ & $98.7 \%$ \\
\hline Absorption correction & Semi-empirical from equivalents \\
\hline Max. and min. transmission & 0.7508 and 0.5953 \\
\hline Refinement method & Full-matrix least-squares on $\mathrm{F}^{2}$ \\
\hline Data / restraints / parameters & $2950 / 0 / 211$ \\
\hline Goodness-of-fit on $\mathrm{F}^{2}$ & 1.054 \\
\hline Final $R$ indices [I $>2 \operatorname{sigma}(\mathrm{I})]$ & $\mathrm{R} 1=0.0317, \mathrm{wR} 2=0.0815$ \\
\hline $\mathrm{R}$ indices (all data) & $\mathrm{R} 1=0.0329, \mathrm{wR} 2=0.0825$ \\
\hline Absolute structure parameter & $0.10(8)$ \\
\hline Extinction coefficient & $\mathrm{n} / \mathrm{a}$ \\
\hline Largest diff. peak and hole & 0.230 and -0.185 e. $\AA^{-3}$ \\
\hline
\end{tabular}


Table S6. X-ray Crystallographic Data for Fortunolide A (18 $)^{a}$

\begin{tabular}{|c|c|}
\hline Empirical formula & $\mathrm{C}_{19} \mathrm{H}_{20} \mathrm{O}_{4}$ \\
\hline Formula weight & 312.35 \\
\hline Temperature & $170 \mathrm{~K}$ \\
\hline Wavelength & $1.34139 \AA$ \\
\hline Crystal system & Orthorhombic \\
\hline Space group & $\mathrm{P} 22_{1} 2_{1}$ \\
\hline \multirow[t]{3}{*}{ Unit cell dimensions } & $\mathrm{a}=10.0126(3) \AA, \alpha=90^{\circ}$. \\
\hline & $\mathrm{b}=11.8286(4) \AA ̊ \AA, \beta=90^{\circ}$. \\
\hline & $\mathrm{c}=12.3462(4) \AA, \gamma=90^{\circ}$. \\
\hline Volume & $1462.22(8) \AA^{3}$ \\
\hline $\mathrm{Z}$ & 4 \\
\hline Density (calculated) & $1.419 \mathrm{Mg} / \mathrm{m}^{3}$ \\
\hline Absorption coefficient & $0.516 \mathrm{~mm}^{-1}$ \\
\hline $\mathrm{F}(000)$ & 664 \\
\hline Crystal size & $0.12 \times 0.08 \times 0.05 \mathrm{~mm}^{3}$ \\
\hline Theta range for data collection & 4.504 to $54.887^{\circ}$ \\
\hline Index ranges & $-10<=\mathrm{h}<=12,-10<=\mathrm{k}<=14,-7<=\mathrm{l}<=15$ \\
\hline Reflections collected & 10297 \\
\hline Independent reflections & $2740[\mathrm{R}(\mathrm{int})=0.0367]$ \\
\hline Completeness to theta $=53.594^{\circ}$ & $99.8 \%$ \\
\hline Absorption correction & Semi-empirical from equivalents \\
\hline Max. and min. transmission & 0.7508 and 0.5964 \\
\hline Refinement method & Full-matrix least-squares on $\mathrm{F}^{2}$ \\
\hline Data / restraints / parameters & $2740 / 0 / 219$ \\
\hline Goodness-of-fit on $\mathrm{F}^{2}$ & 1.107 \\
\hline Final $R$ indices [I>2sigma $(\mathrm{I})]$ & $\mathrm{R} 1=0.0304, \mathrm{wR} 2=0.0713$ \\
\hline $\mathrm{R}$ indices (all data) & $\mathrm{R} 1=0.0332, \mathrm{wR} 2=0.0733$ \\
\hline Absolute structure parameter & $0.02(11)$ \\
\hline Extinction coefficient & $\mathrm{n} / \mathrm{a}$ \\
\hline Largest diff. peak and hole & 0.161 and -0.150 e. $\AA^{-3}$ \\
\hline
\end{tabular}

${ }^{a}$ Crystals of 18 were obtained from methanol. 
Table S7. X-ray Crystallographic Data for 14-epi-Cephafortoid A (24) ${ }^{a}$

\begin{tabular}{|c|c|}
\hline Empirical formula & $\mathrm{C}_{19} \mathrm{H}_{28} \mathrm{O}_{7}$ \\
\hline Formula weight & 368.41 \\
\hline Temperature & $169.96 \mathrm{~K}$ \\
\hline Wavelength & $1.34139 \AA$ \\
\hline Crystal system & Monoclinic \\
\hline Space group & C 121 \\
\hline \multirow[t]{3}{*}{ Unit cell dimensions } & $\mathrm{a}=18.8453(3) \AA, \alpha=90^{\circ}$ \\
\hline & $\mathrm{b}=10.1003(2) \AA, \beta=106.5500(10)^{\circ}$ \\
\hline & $\mathrm{c}=9.7629(2) \AA, \gamma=90^{\circ}$ \\
\hline Volume & $1781.31(6) \AA^{3}$ \\
\hline $\mathrm{Z}$ & 4 \\
\hline Density (calculated) & $1.374 \mathrm{Mg} / \mathrm{m}^{3}$ \\
\hline Absorption coefficient & $0.555 \mathrm{~mm}^{-1}$ \\
\hline $\mathrm{F}(000)$ & 792 \\
\hline Crystal size & $0.2 \times 0.18 \times 0.15 \mathrm{~mm}^{3}$ \\
\hline Theta range for data collection & 8.129 to $54.853^{\circ}$. \\
\hline Index ranges & $-22<=\mathrm{h}<=22,-12<=\mathrm{k}<=12,-11<=\mathrm{l}<=11$ \\
\hline Reflections collected & 11486 \\
\hline Independent reflections & $3240[\mathrm{R}(\mathrm{int})=0.0282]$ \\
\hline Completeness to theta $=53.594^{\circ}$ & $95.9 \%$ \\
\hline Absorption correction & Semi-empirical from equivalents \\
\hline Max. and min. transmission & 0.7508 and 0.6018 \\
\hline Refinement method & Full-matrix least-squares on $\mathrm{F}^{2}$ \\
\hline Data / restraints / parameters & $3240 / 1 / 253$ \\
\hline Goodness-of-fit on $\mathrm{F}^{2}$ & 1.067 \\
\hline Final $\mathrm{R}$ indices [I>2sigma $(\mathrm{I})]$ & $\mathrm{R} 1=0.0280, \mathrm{wR} 2=0.0743$ \\
\hline $\mathrm{R}$ indices (all data) & $\mathrm{R} 1=0.0281, \mathrm{wR} 2=0.0744$ \\
\hline Absolute structure parameter & $0.07(4)$ \\
\hline Extinction coefficient & $\mathrm{n} / \mathrm{a}$ \\
\hline Largest diff. peak and hole & 0.194 and -0.252 e. $\AA^{-3}$ \\
\hline
\end{tabular}

${ }^{a}$ Crystals of $\mathbf{2 4}$ were obtained from methanol. 
Table S8. Cytotoxicity of Cephalotaxus Troponoids against Tumor Cell Lines ( $\mathrm{IC}_{50}$ in $\mu \mathrm{M}$ )

\begin{tabular}{ccc}
\hline \hline & A-549 & HL-60 \\
\hline $\mathbf{1}$ & $10.3 \pm 2.3$ & $\mathrm{NA}^{a}$ \\
$\mathbf{2}$ & $25.4 \pm 11.0$ & $38.4 \pm 12.8$ \\
$\mathbf{3}$ & $22.0 \pm 1.4$ & $\mathrm{NA}^{a}$ \\
$\mathbf{5}$ & $4.5 \pm 0.1$ & $6.8 \pm 0.9$ \\
$\mathbf{6}$ & $2.4 \pm 0.1$ & $2.2 \pm 0.3$ \\
$\mathbf{8}$ & $1.0 \pm 0.1$ & $9.8 \pm 3.6$ \\
$\mathbf{1 0}$ & $11.5 \pm 1.9$ & $17.2 \pm 5.6$ \\
$\mathbf{1 9}$ & $2.4 \pm 0.6$ & $3.5 \pm 0.1$ \\
$\mathbf{2 1}$ & $5.7 \pm 1.6$ & $20.0 \pm 1.7$ \\
$\mathrm{Adr}{ }^{b}$ & $0.44 \pm 0.10$ & $0.075 \pm 0.013$ \\
${ }^{a} \mathrm{IC}_{50}>50 \mu \mathrm{M}$ was considered inactive (NA). \\
${ }^{b} \mathrm{Adr}$ (adriamycin) was used as positive control. \\
\hline \multicolumn{3}{c}{} \\
\hline
\end{tabular}


a)

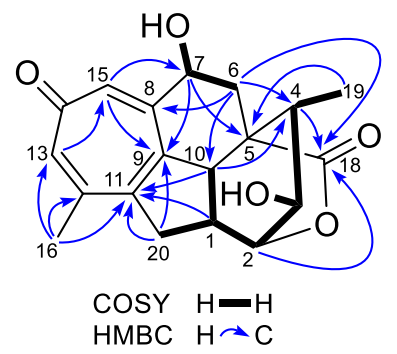

b)

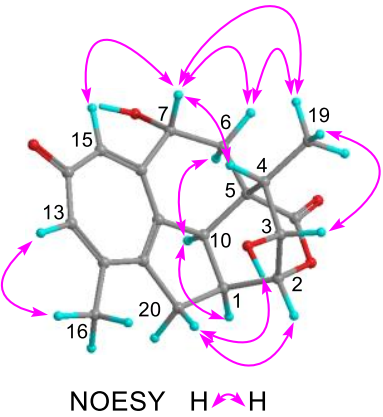

Figure S1. Selected 2D NMR correlations of compound 2.

a)

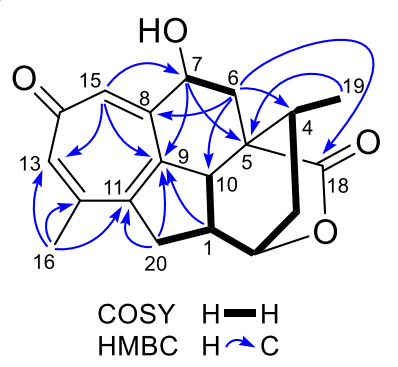

b)

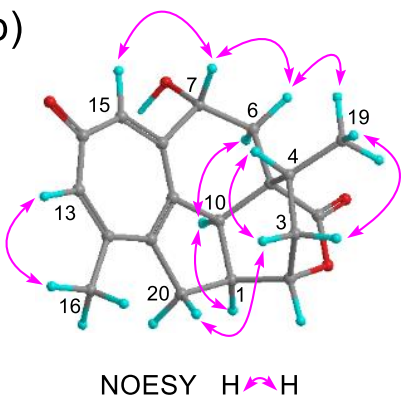

Figure S2. Selected 2D NMR correlations of compound 3.

a)

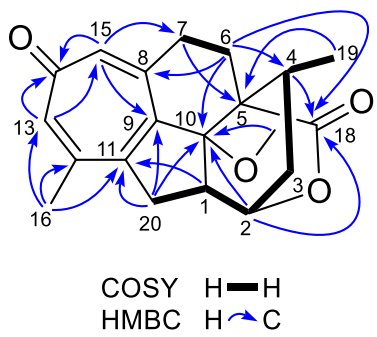

b)

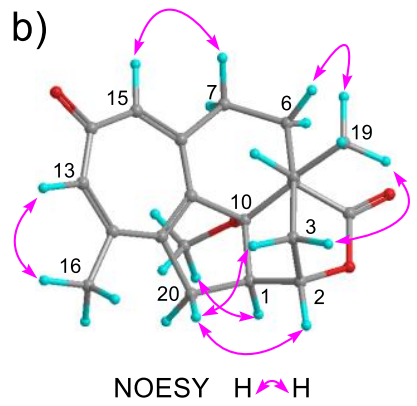

Figure S3. Selected 2D NMR correlations of compound 4. 
a)

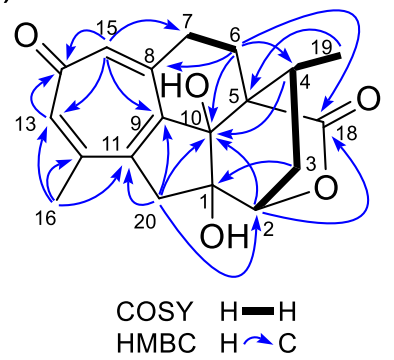

b)

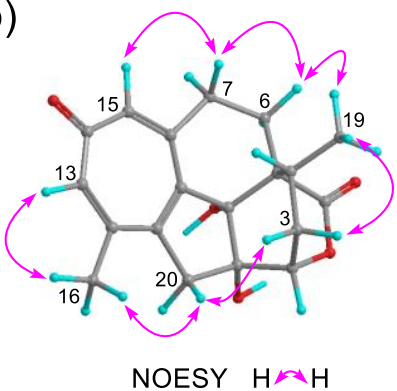

Figure S4. Selected 2D NMR correlations of compound 5.

a)

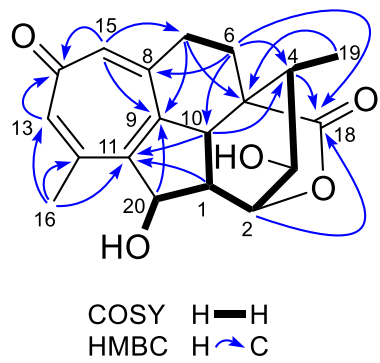

b)

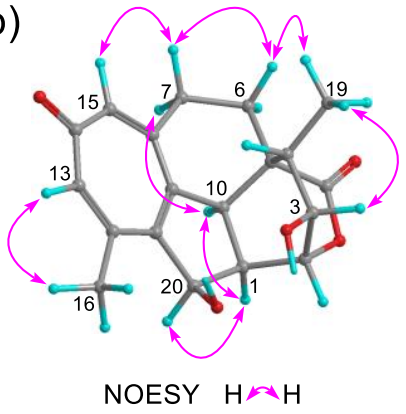

Figure S5. Selected 2D NMR correlations of compound 6.

a)

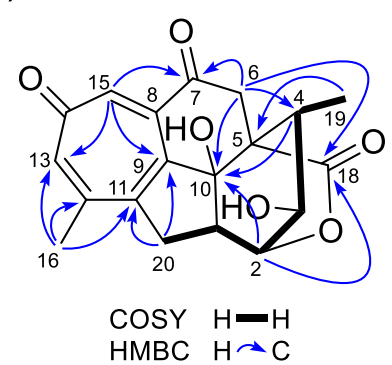

b)

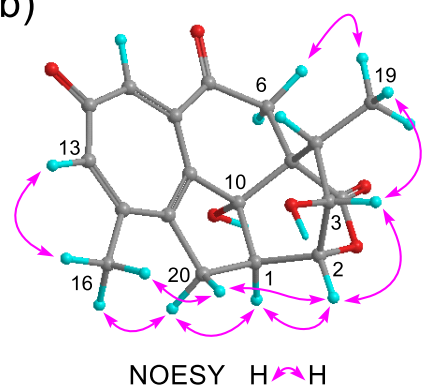

Figure S6. Selected 2D NMR correlations of compound 7. 

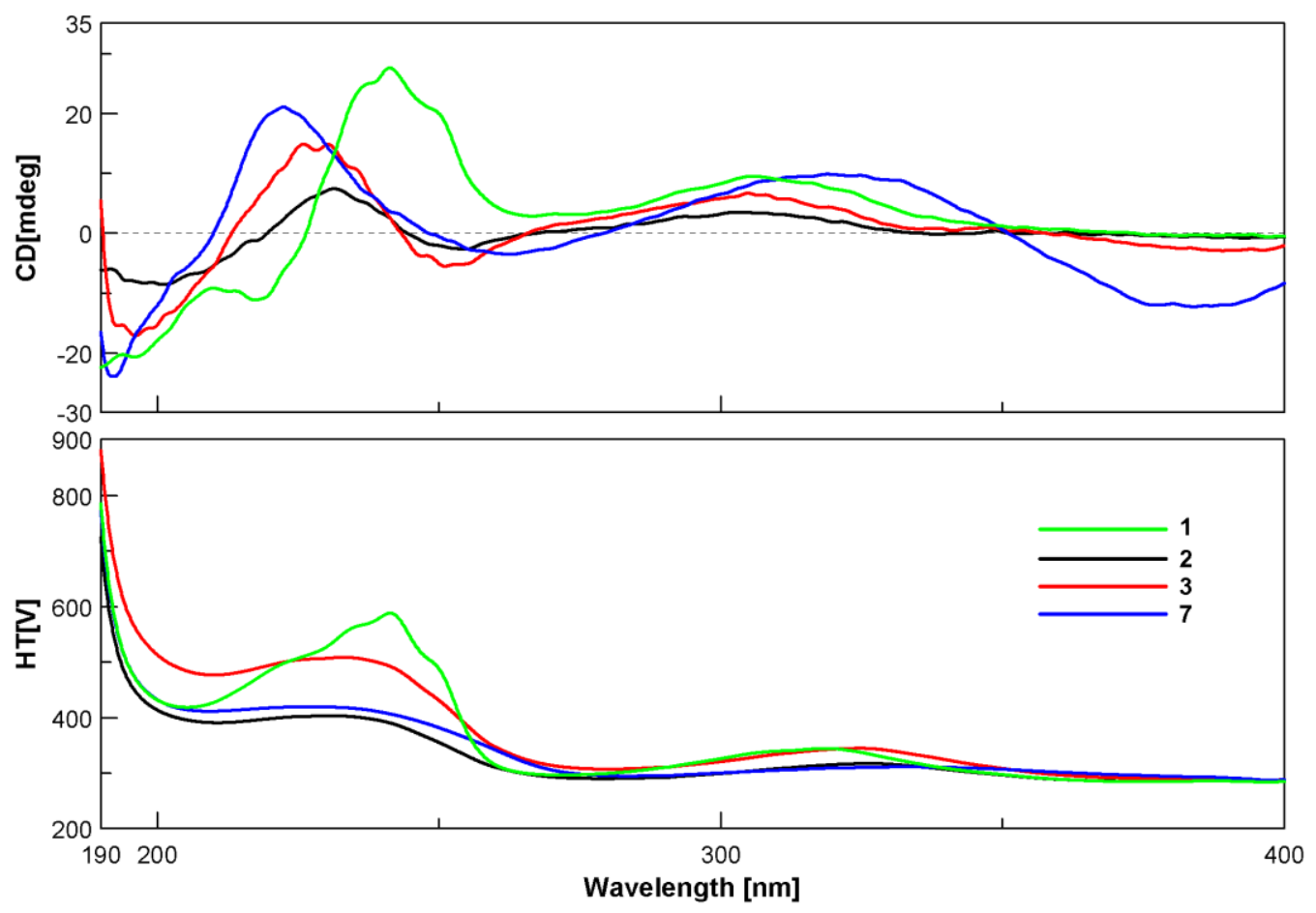

Figure S7. ECD spectra of compounds 1, 2, 3, and $\mathbf{7}$

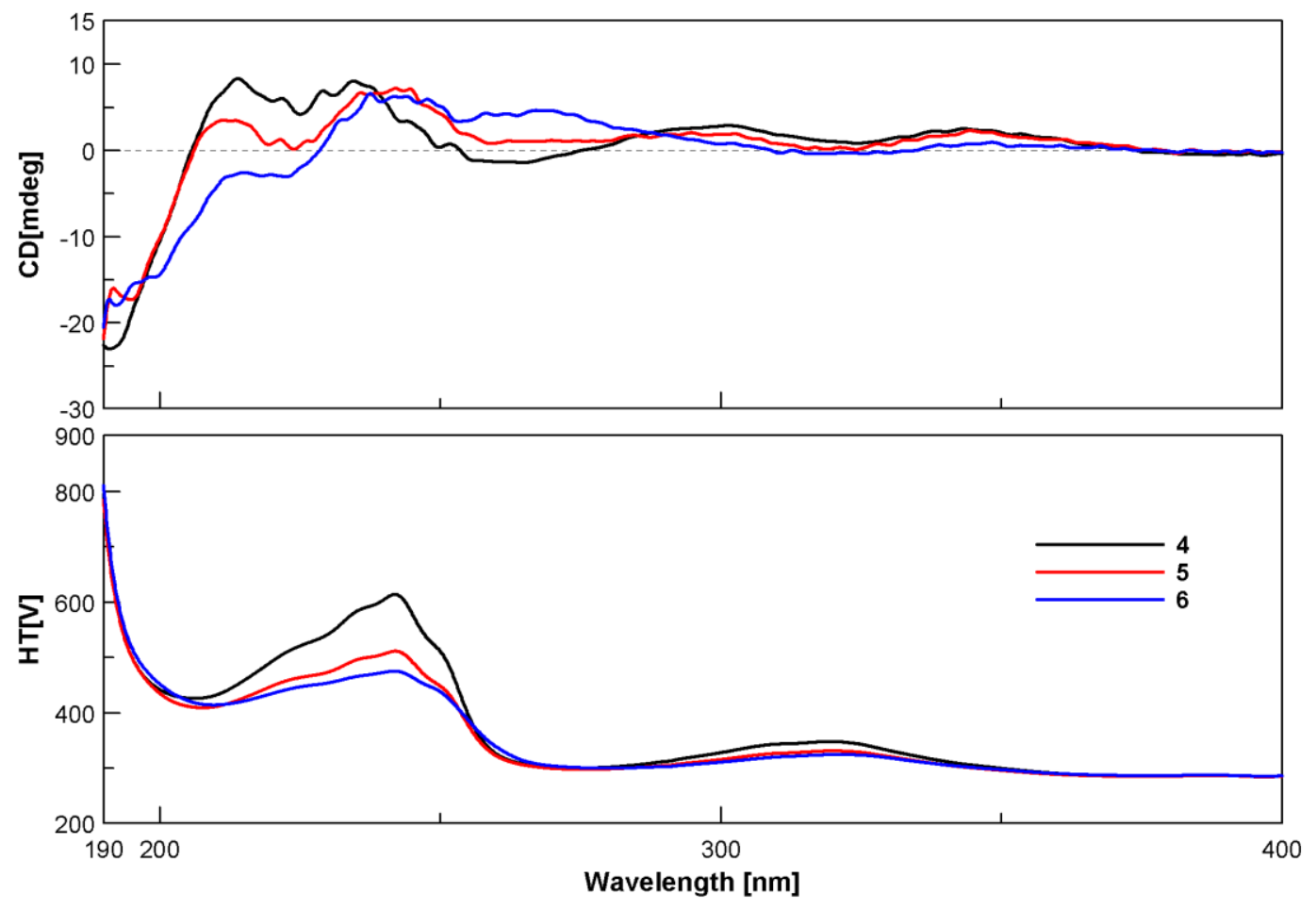

Figure S8. ECD spectra of compounds 4, 5, and $\mathbf{6}$ 
a)

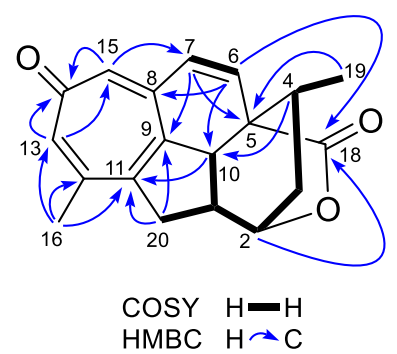

b)

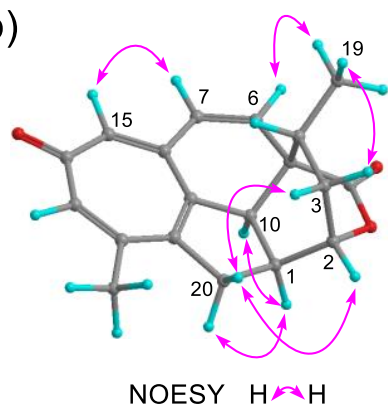

Figure S9. Selected 2D NMR correlations of compound 8.

a)

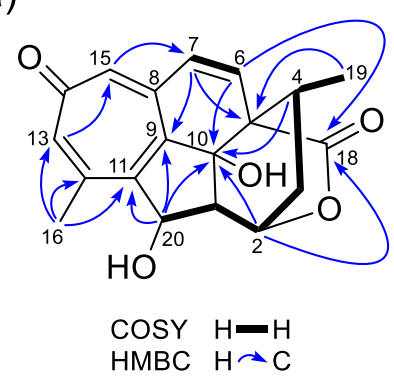

b)

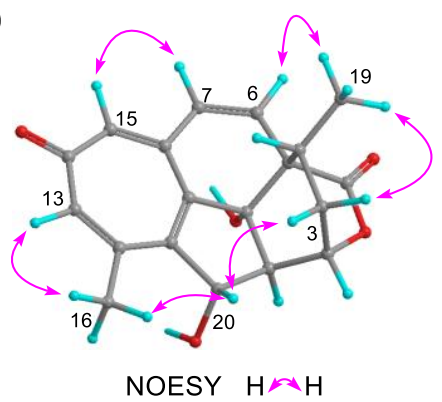

Figure S10. Selected 2D NMR correlations of compound 9.

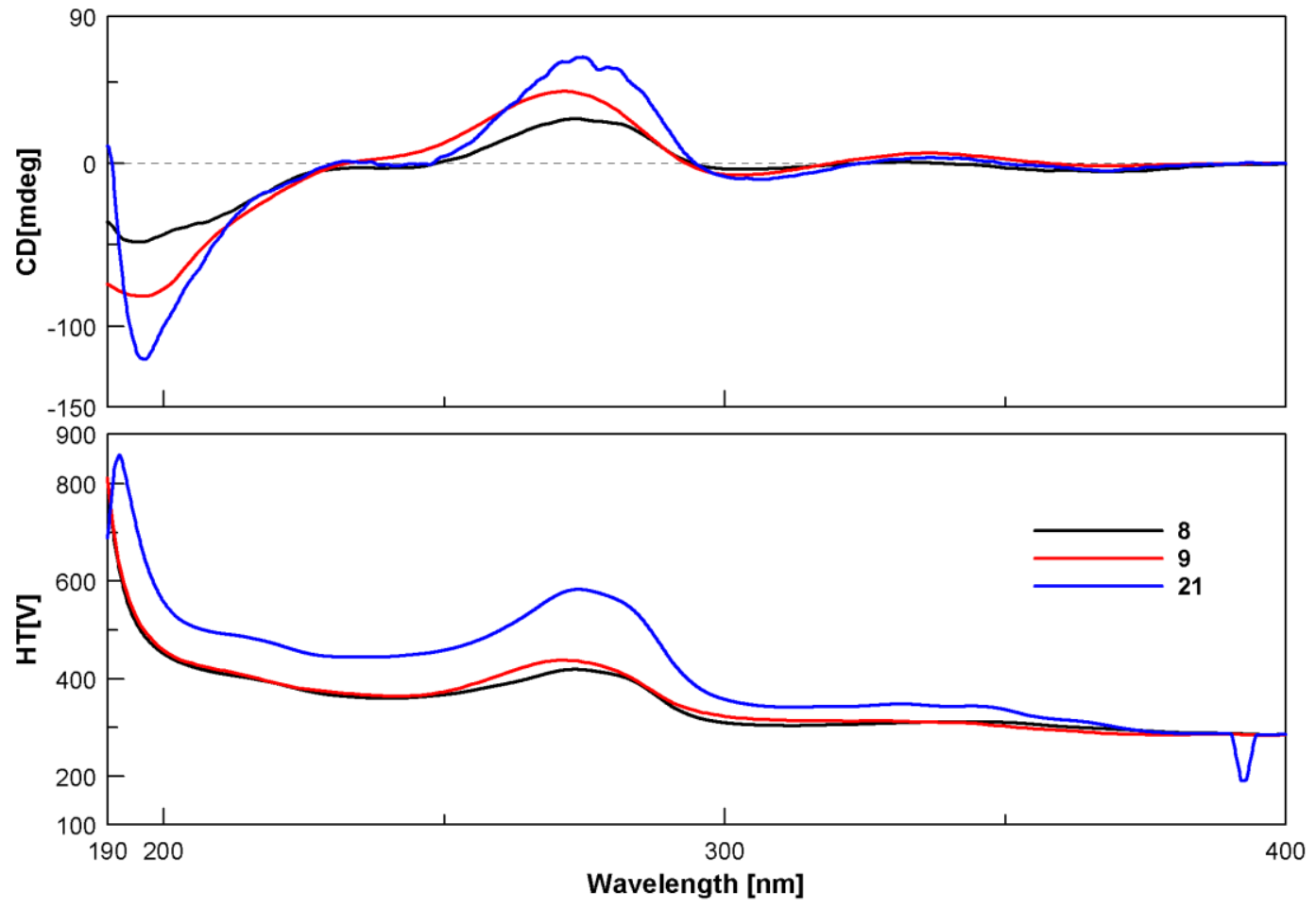

Figure S11. ECD spectra of compounds 8, 9, and 21 
a)

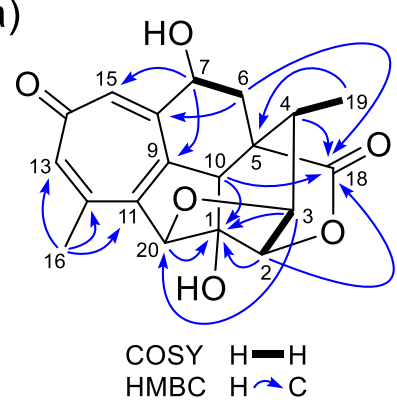

b)

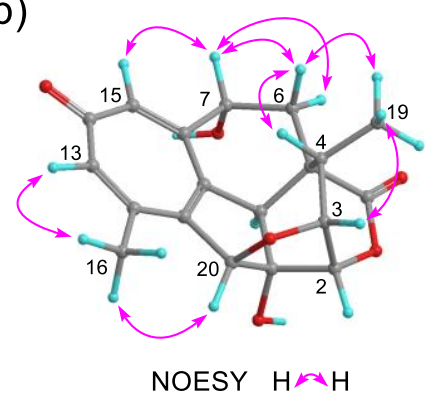

Figure S12. Selected 2D NMR correlations of compound $\mathbf{1 0 .}$

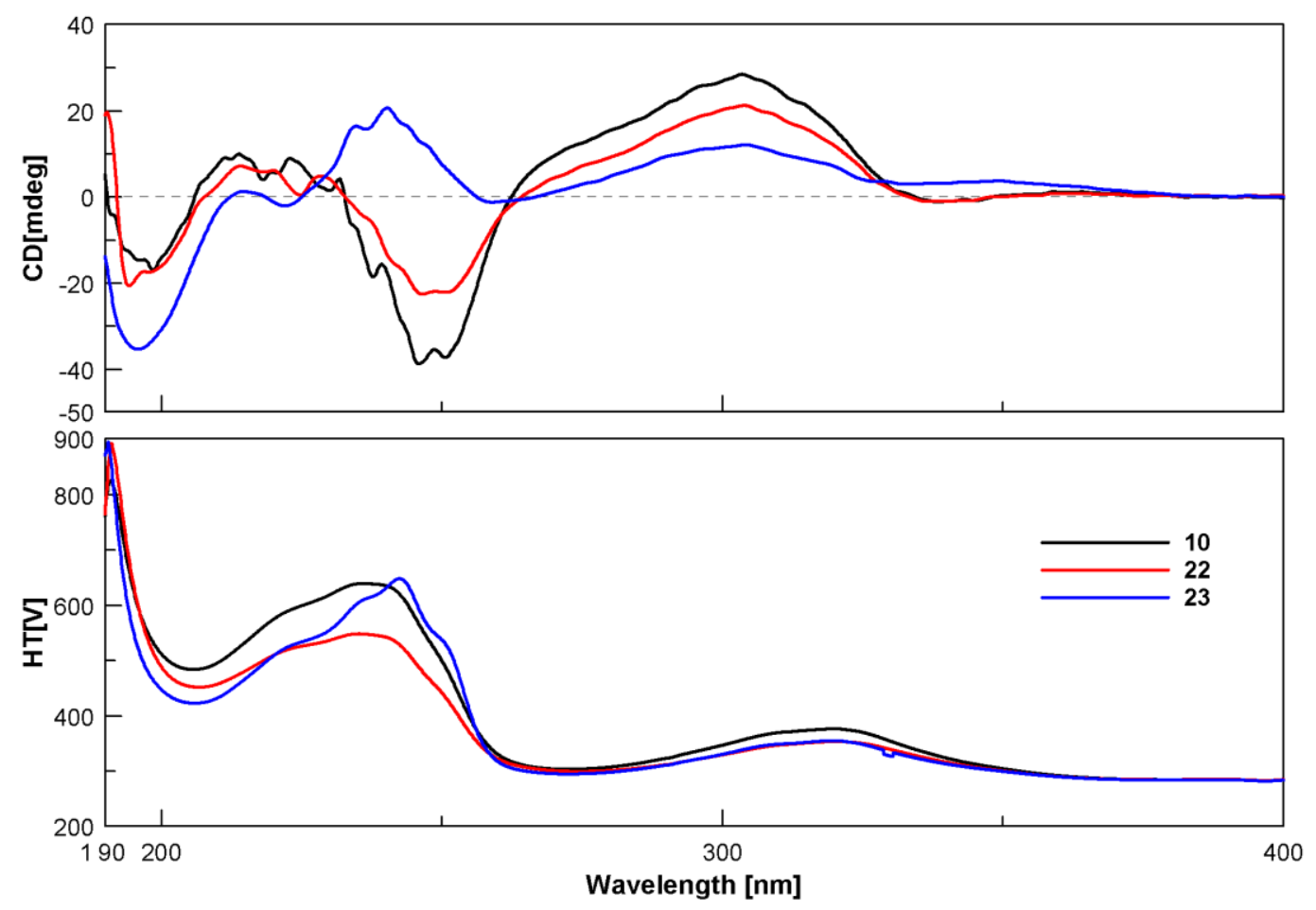

Figure S13. ECD spectra of compounds 10, 22, and 23 
a)

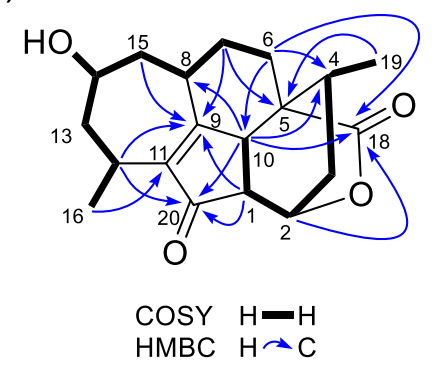

b)

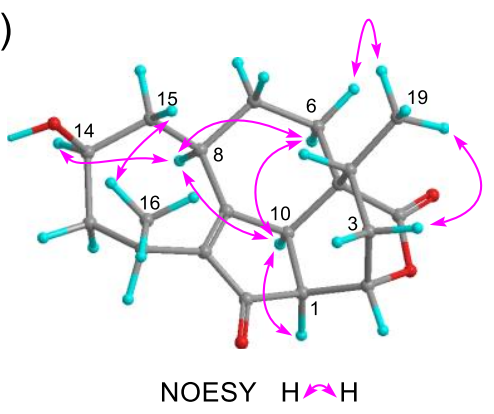

Figure S14. Selected 2D NMR correlations of compound 14.

a)

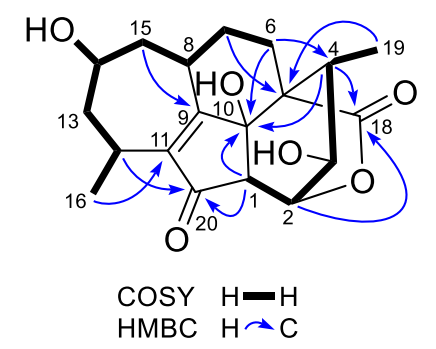

b)

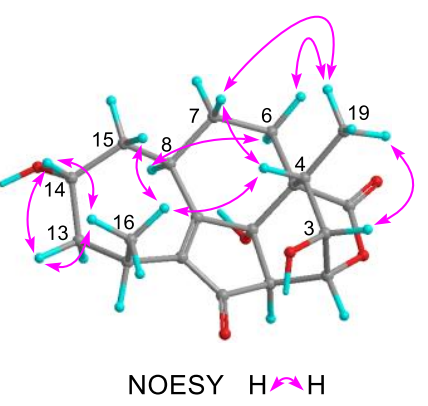

Figure S15. Selected 2D NMR correlations of compound 15.

a)

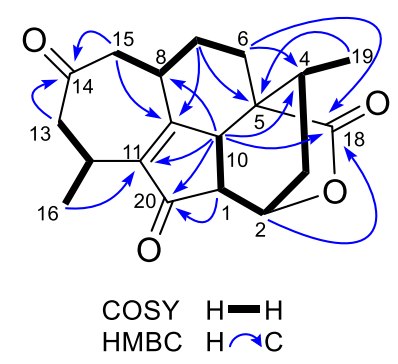

b)

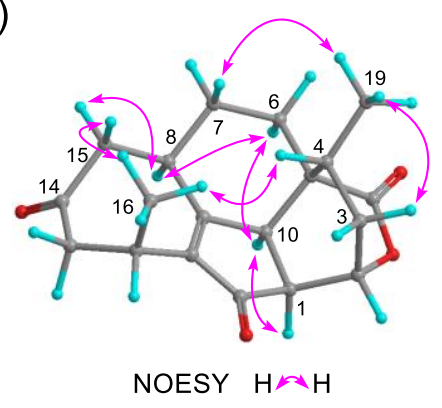

Figure S16. Selected 2D NMR correlations of compound 16.

a)

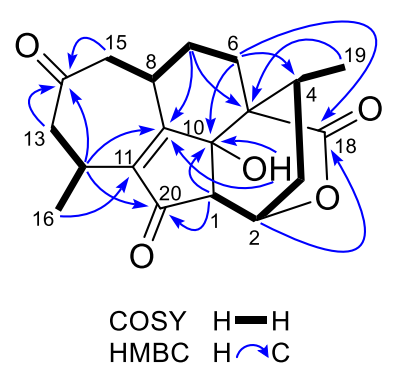

b)

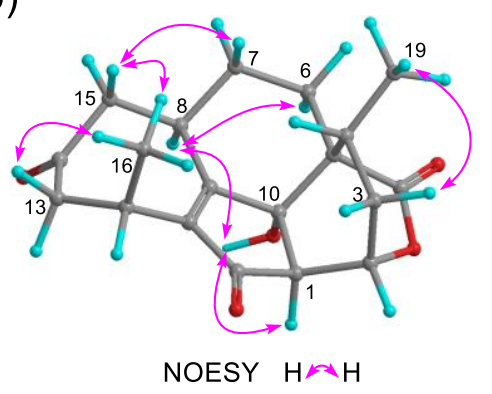

Figure S17. Selected 2D NMR correlations of compound 17. 
Figure S18. ${ }^{1} \mathrm{H}$ NMR spectrum of fortalpinoid $\mathrm{A}(\mathbf{1})$ in $\mathrm{CDCl}_{3}$.
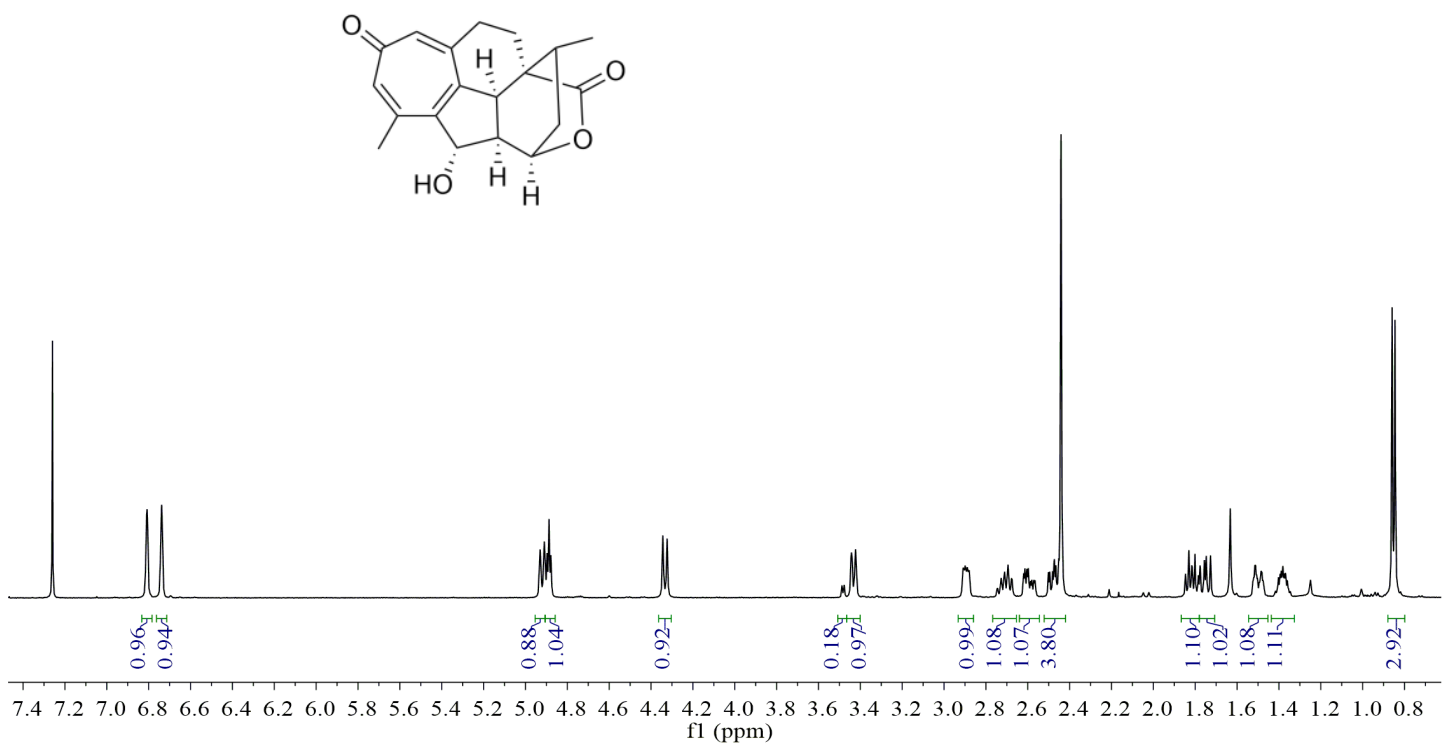

Figure S19. ${ }^{13} \mathrm{C}$ NMR spectrum of fortalpinoid $\mathrm{A}(\mathbf{1})$ in $\mathrm{CDCl}_{3}$.
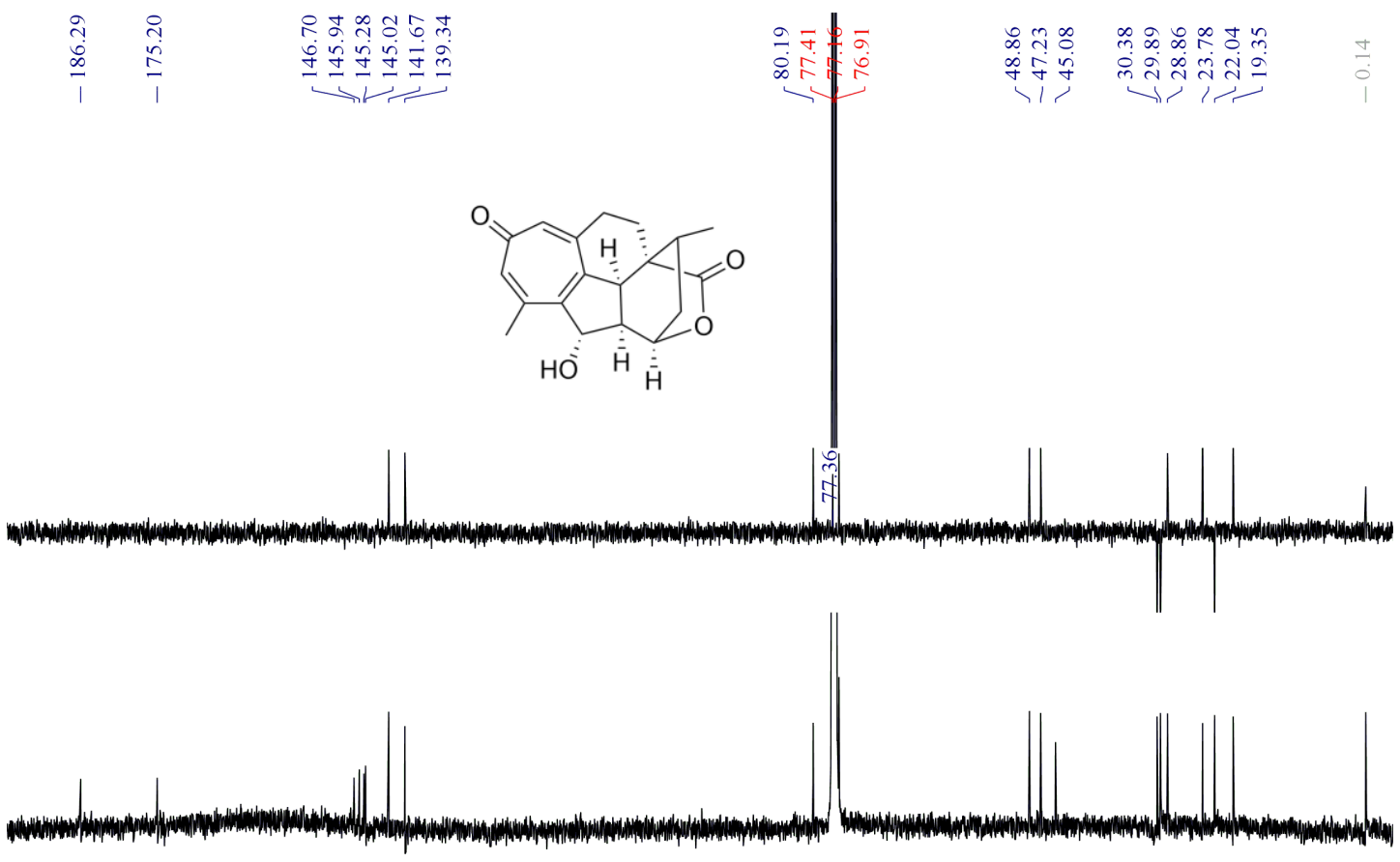

$\begin{array}{lllllllllllllllllll}190 & 180 & 170 & 160 & 150 & 140 & 130 & 120 & 110 & \begin{array}{l}100 \\ \mathrm{fl}(\mathrm{ppm})\end{array} & 80 & 70 & 60 & 50 & 40 & 30 & 20 & 10 & 0\end{array}$ 
Figure S20. HSQC spectrum of fortalpinoid A (1) in CDCl3.

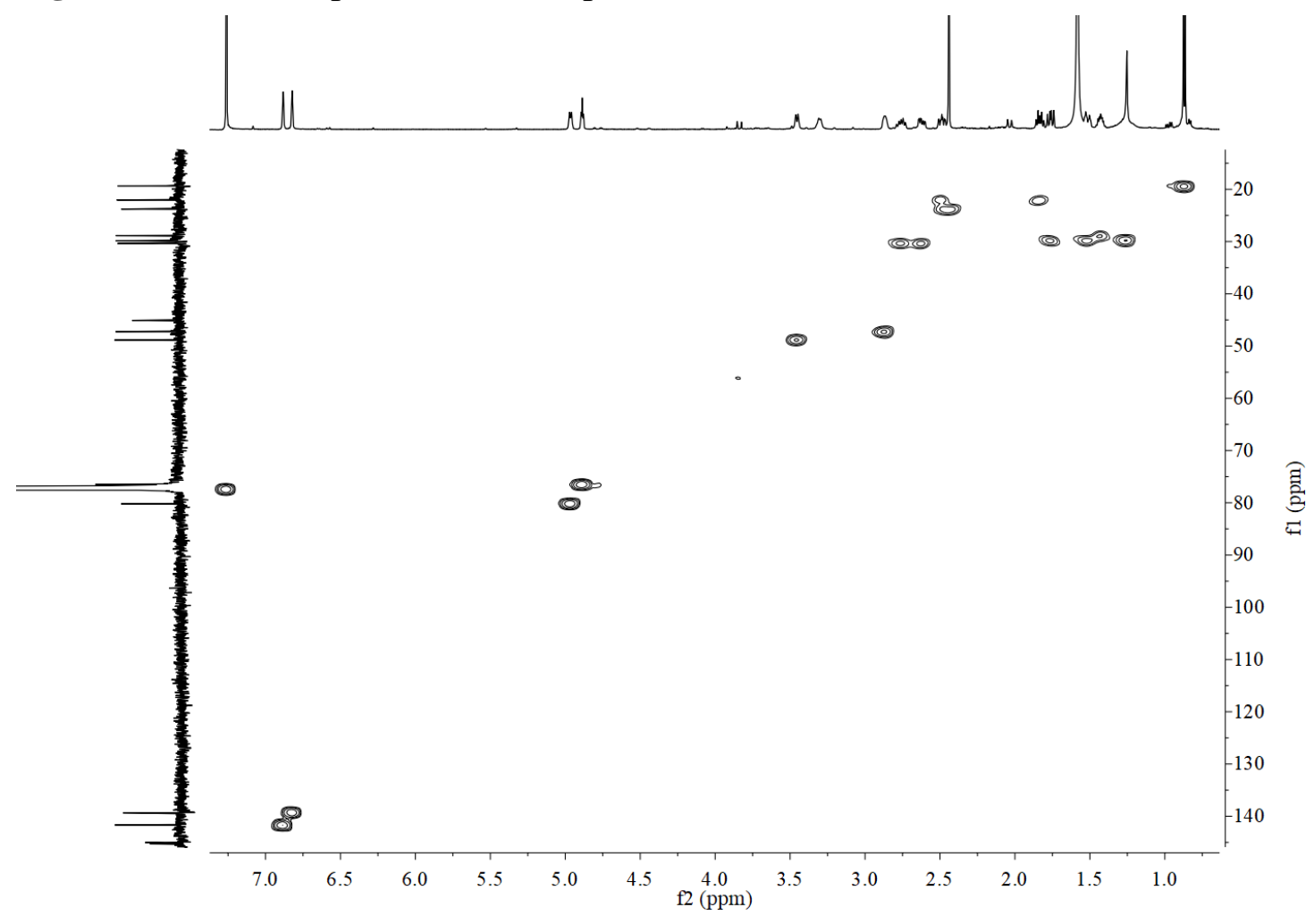

Figure S21. HMBC spectrum of fortalpinoid A (1) in $\mathrm{CDCl}_{3}$.

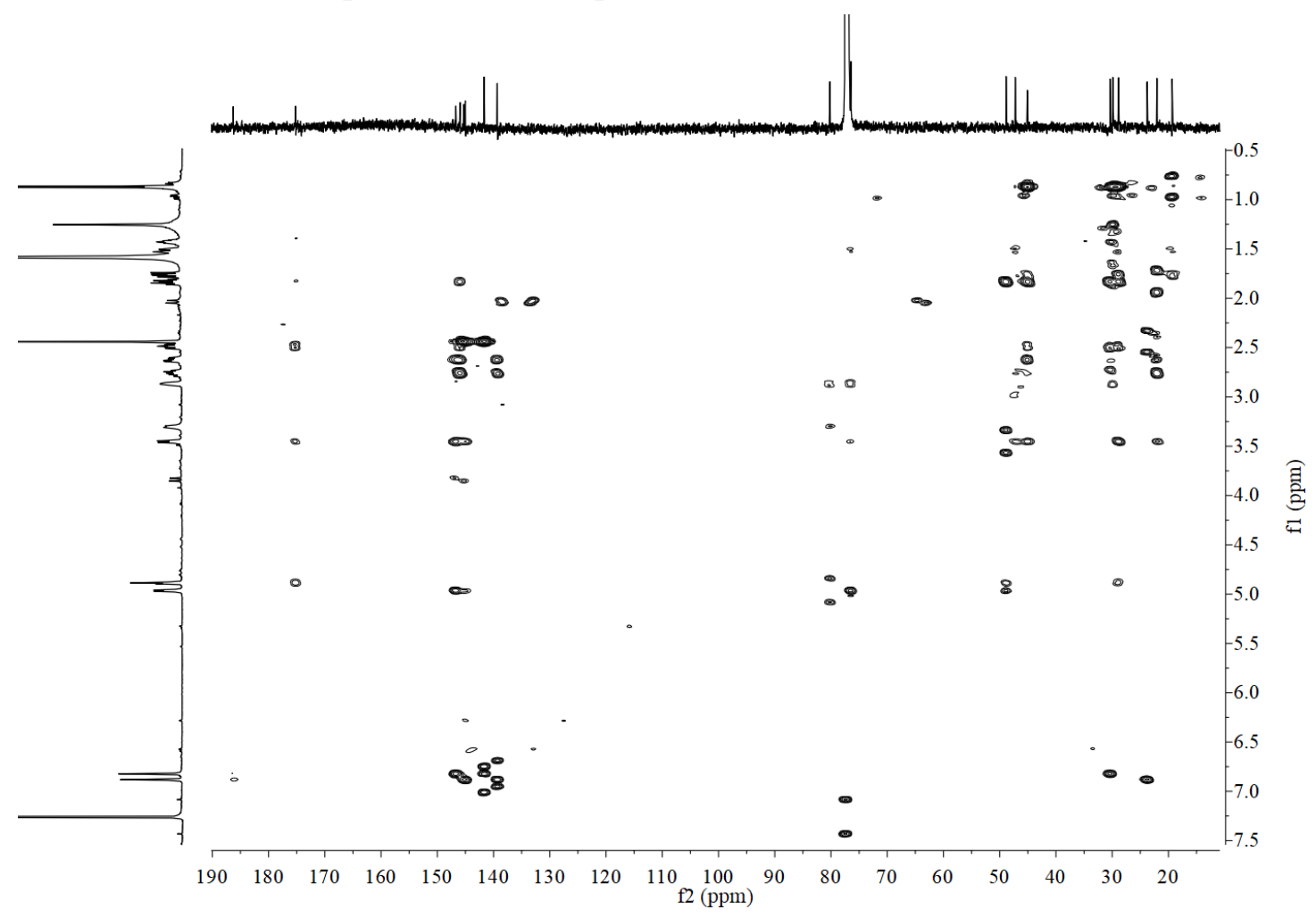


Figure S22. ${ }^{1} \mathrm{H}-{ }^{1} \mathrm{H}$ COSY spectrum of fortalpinoid A (1) in $\mathrm{CDCl}_{3}$.

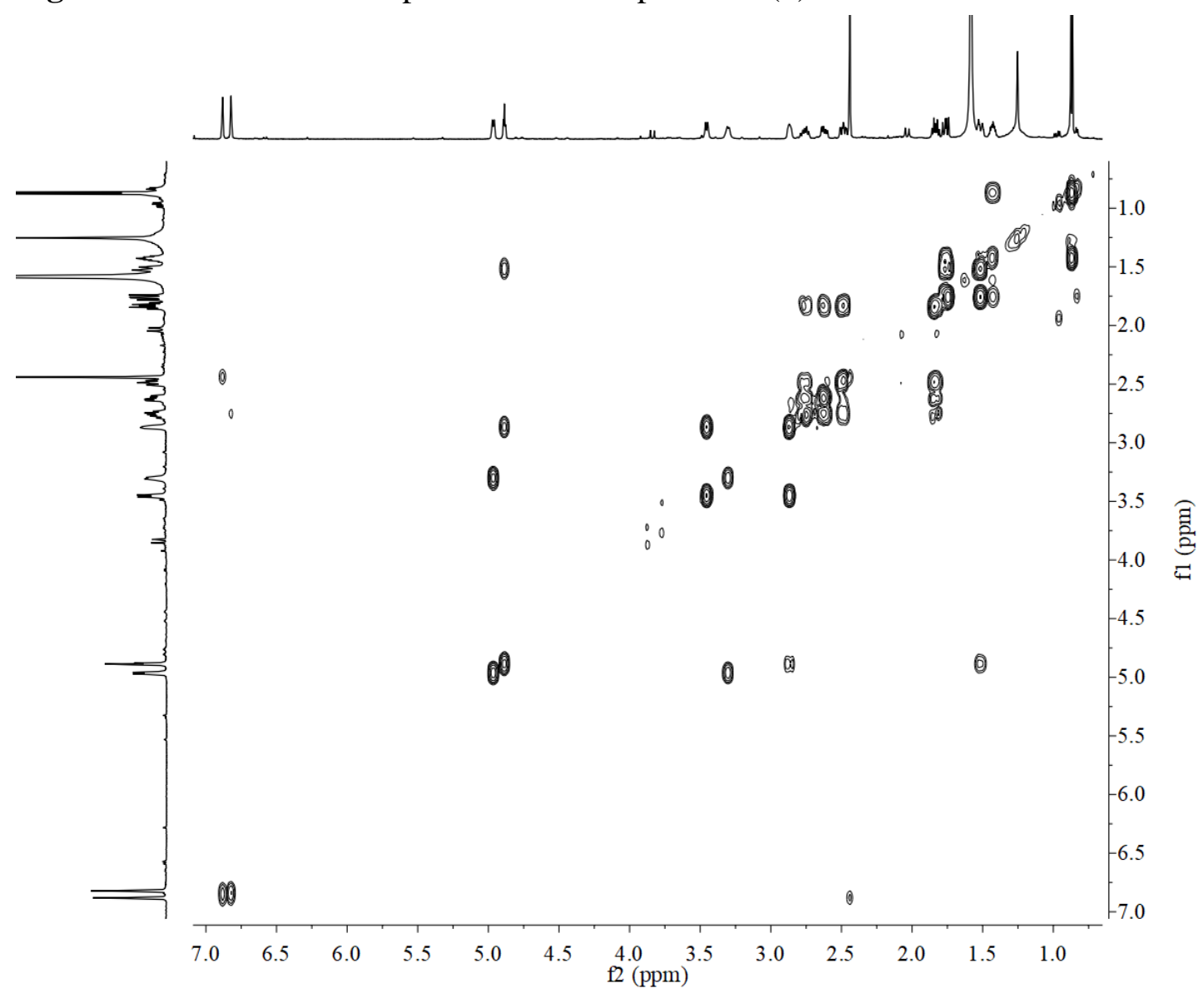

Figure S23. NOESY spectrum of fortalpinoid A (1) in $\mathrm{CDCl}_{3}$.

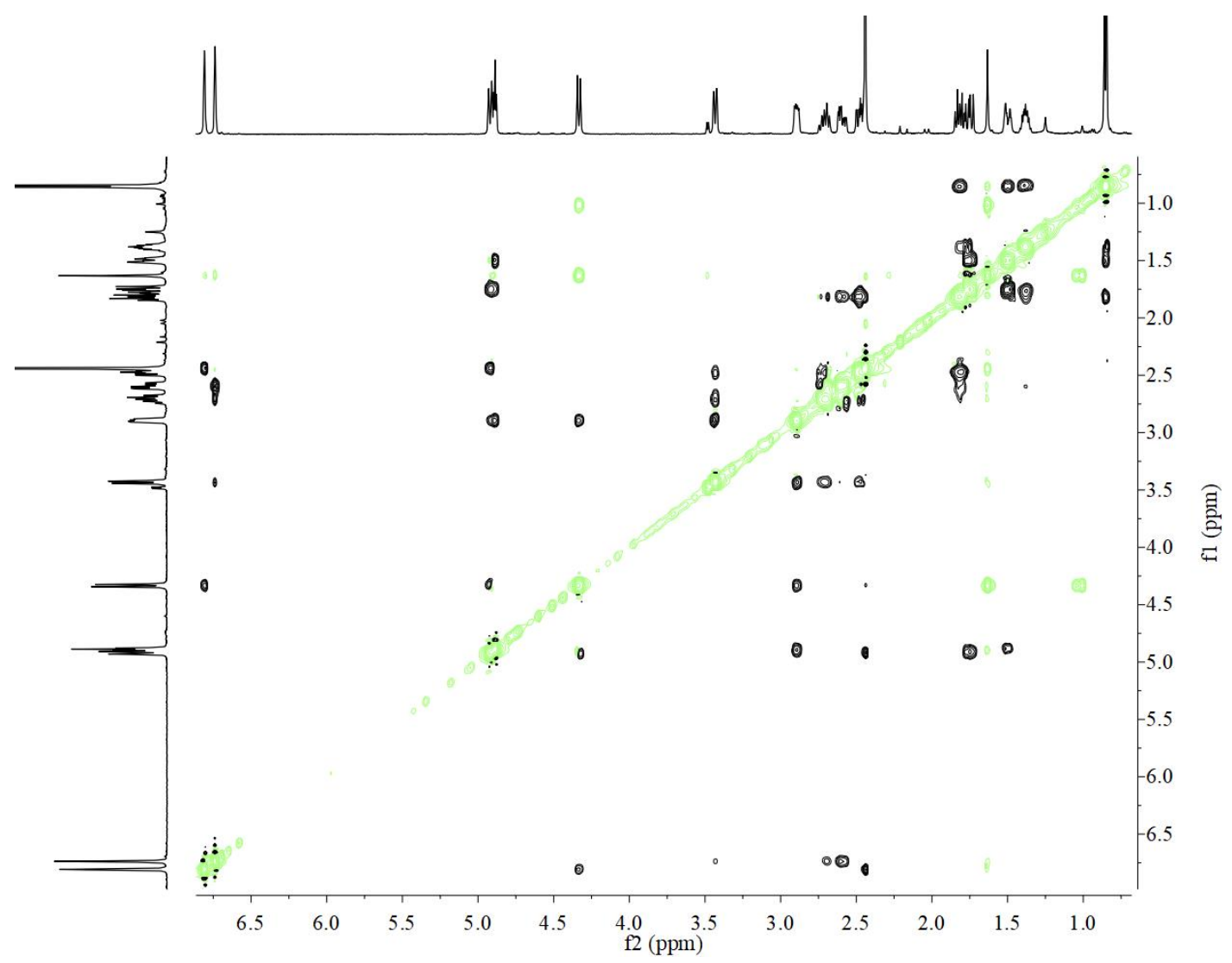


Figure S24. (+)-ESIMS spectrum of fortalpinoid A (1).

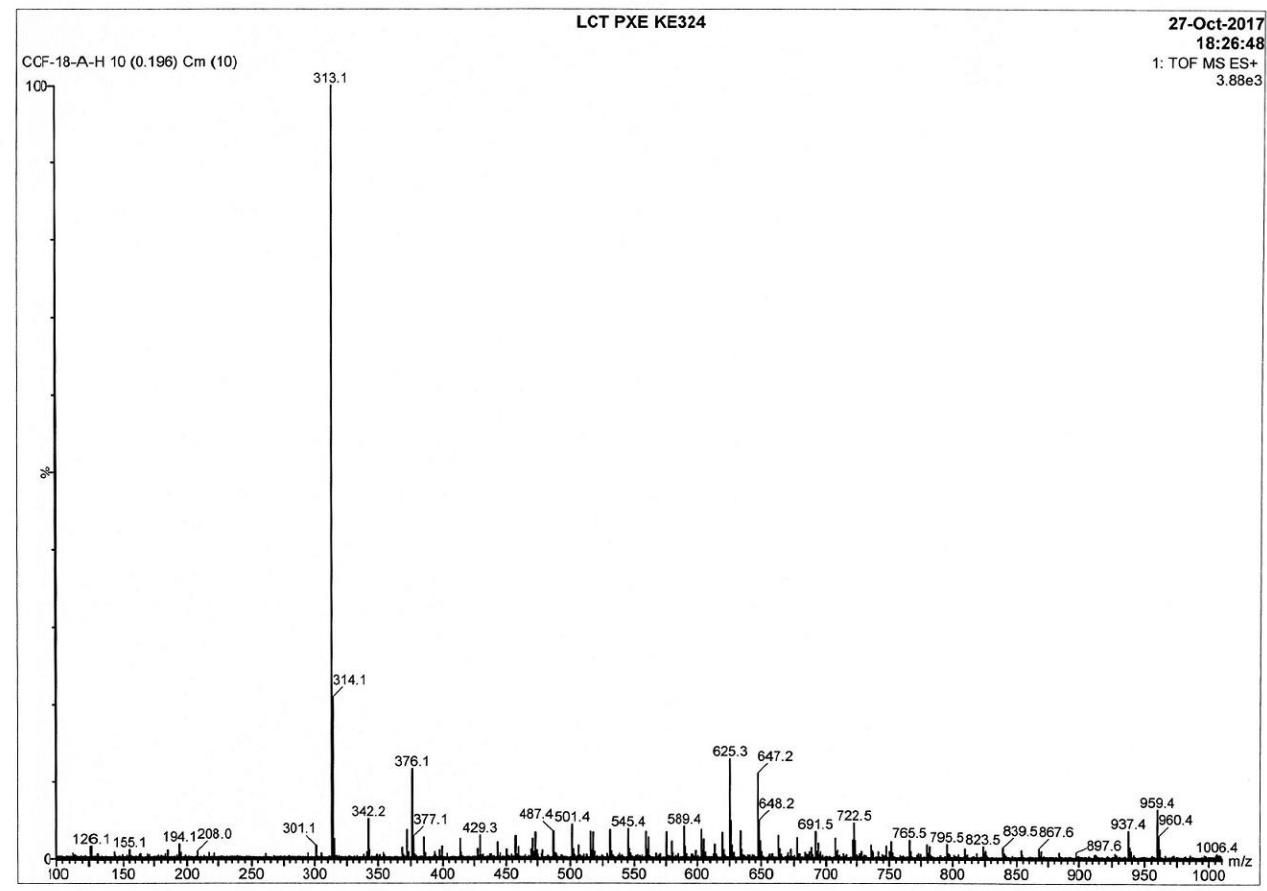

Figure S25. (+)-HRESIMS spectrum of fortalpinoid A (1).

Elemental Composition Report

Page 1

Single Mass Analysis

Tolerance $=3.0$ PPM I DBE: $\min =-1.5, \max =50.0$

Element prediction: Off

Number of isotope peaks used for $\mathrm{i}-\mathrm{FIT}=3$

Monoisotopic Mass, Even Electron Ions

57 formula(e) evaluated with 1 results within limits (up to 50 best isotopic matches for each mass)

$\begin{array}{lll}\text { Elements Used: } & \text { H: } 0-1000 & \text { O: } 0-500\end{array}$

LCT PXE KE 324

27-Oct-2017

CCF-18-A-H 35 (0.742) $\begin{array}{r}\text { 18:26:48 } \\ \text { (34:35) }\end{array}$

1: TOF MS ES+

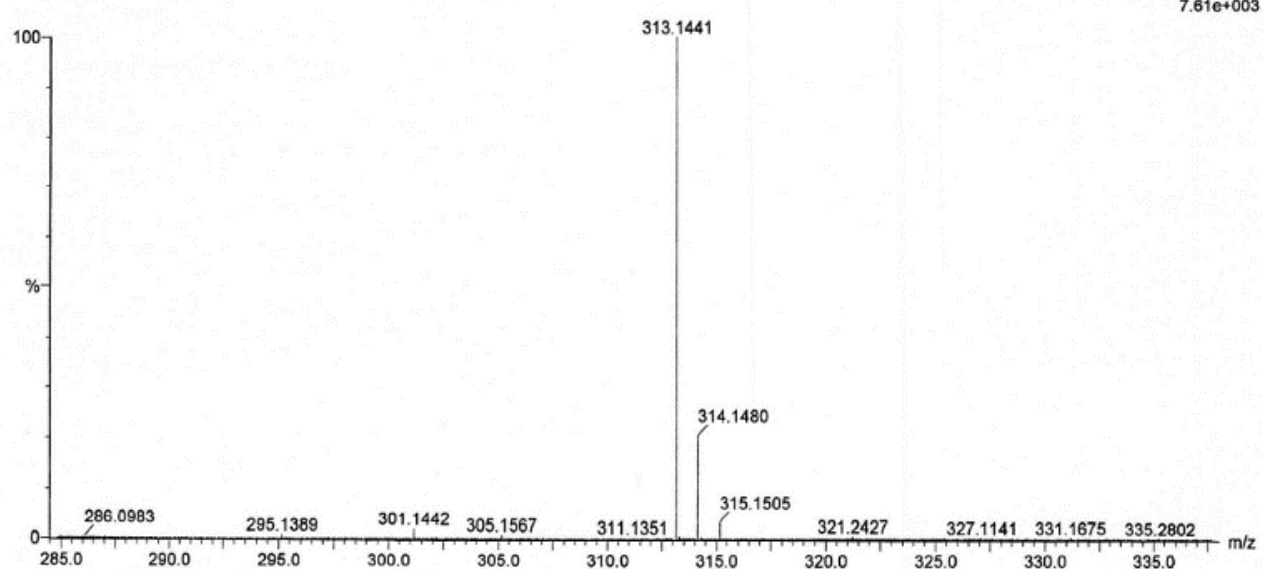

Minimum:

$\begin{array}{lll}5.0 & 3.0 & -1.5 \\ 50.0\end{array}$

Mass Calc. Mass mDa PPM DBE

$313.1441 \quad 313.1440$

0.1

0.3

9.5

i-FIT

i-FIT (Norm) Formula

$224.4 \quad 0.0$

C19 H21 O4 
Figure S26. IR spectrum of fortalpinoid A (1).

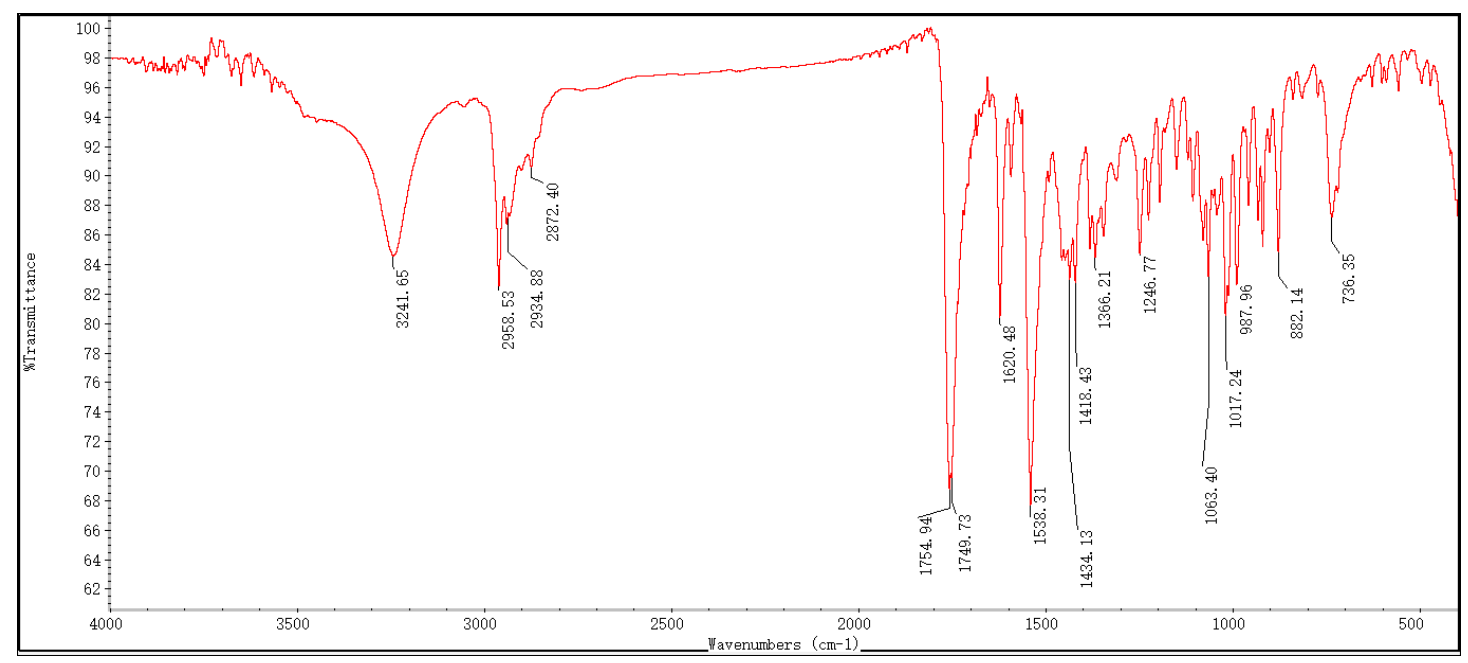

Figure S27. ${ }^{1} \mathrm{H}$ NMR spectrum of fortalpinoid B (2) in pyridine- $d 5$.

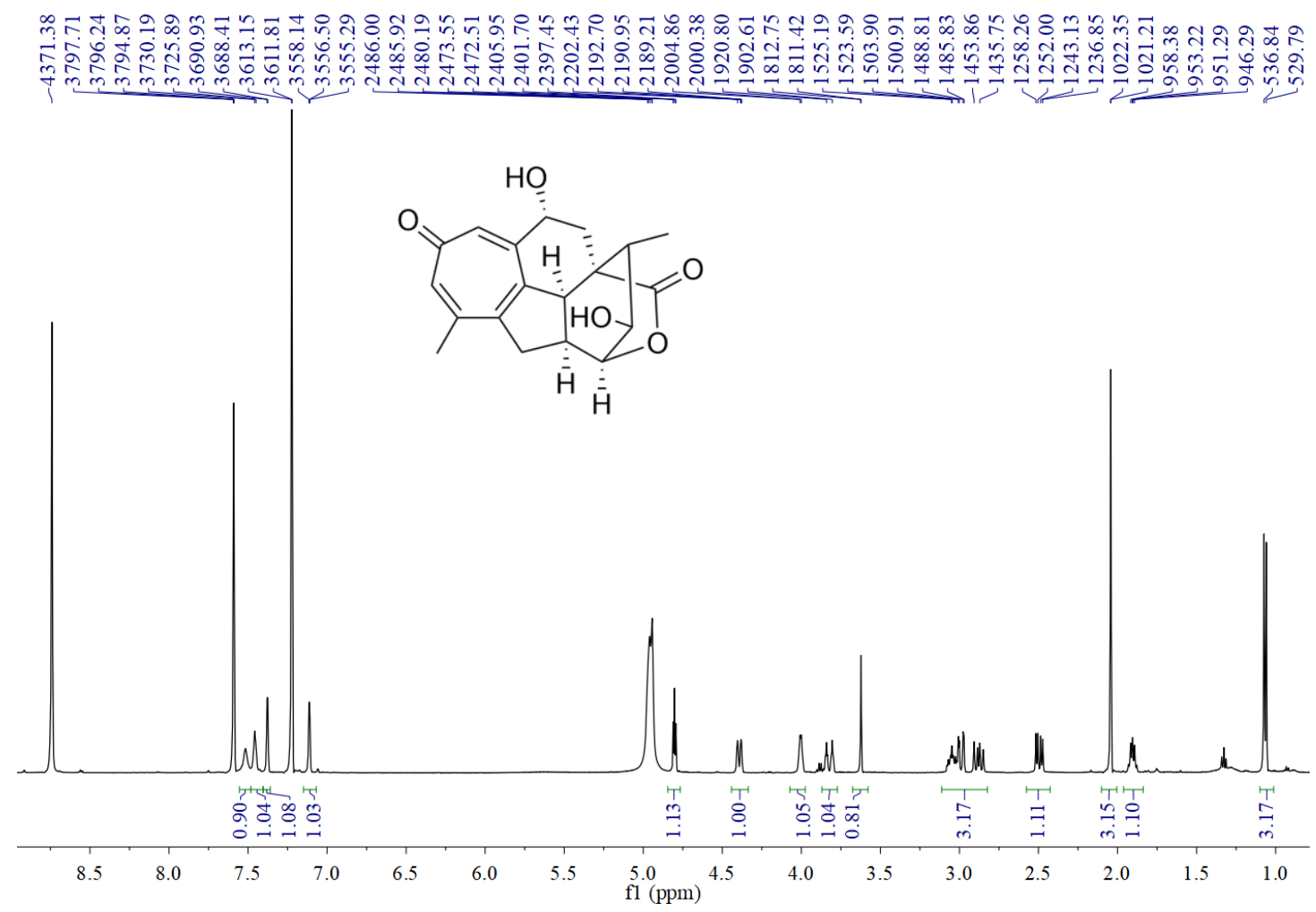


Figure S28. ${ }^{13} \mathrm{C}$ NMR spectrum of fortalpinoid B (2) in pyridine- $d_{5}$.
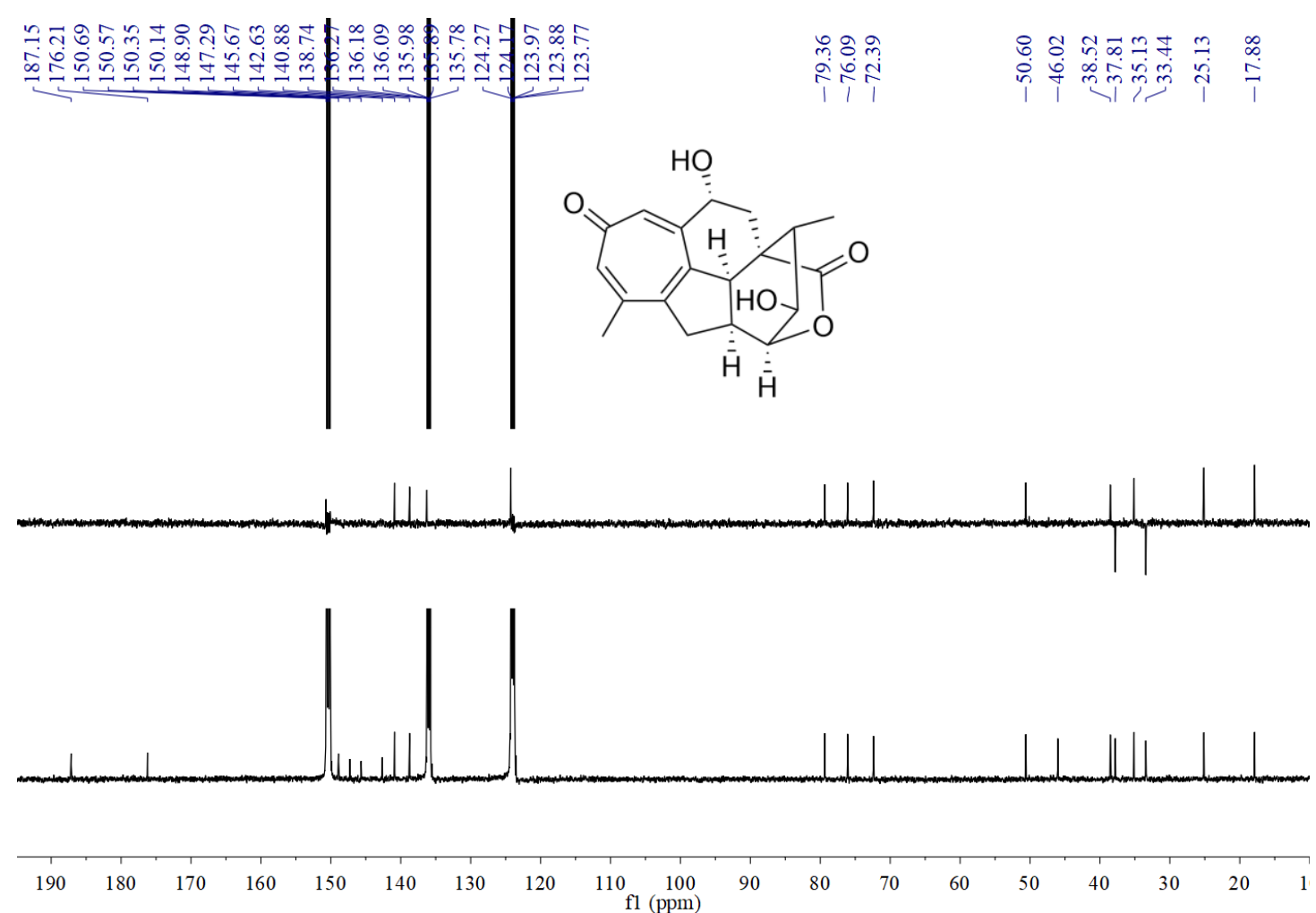

Figure S29. ${ }^{1} \mathrm{H}$ NMR spectrum of fortalpinoid B (2) in methanol- $d_{4}$.

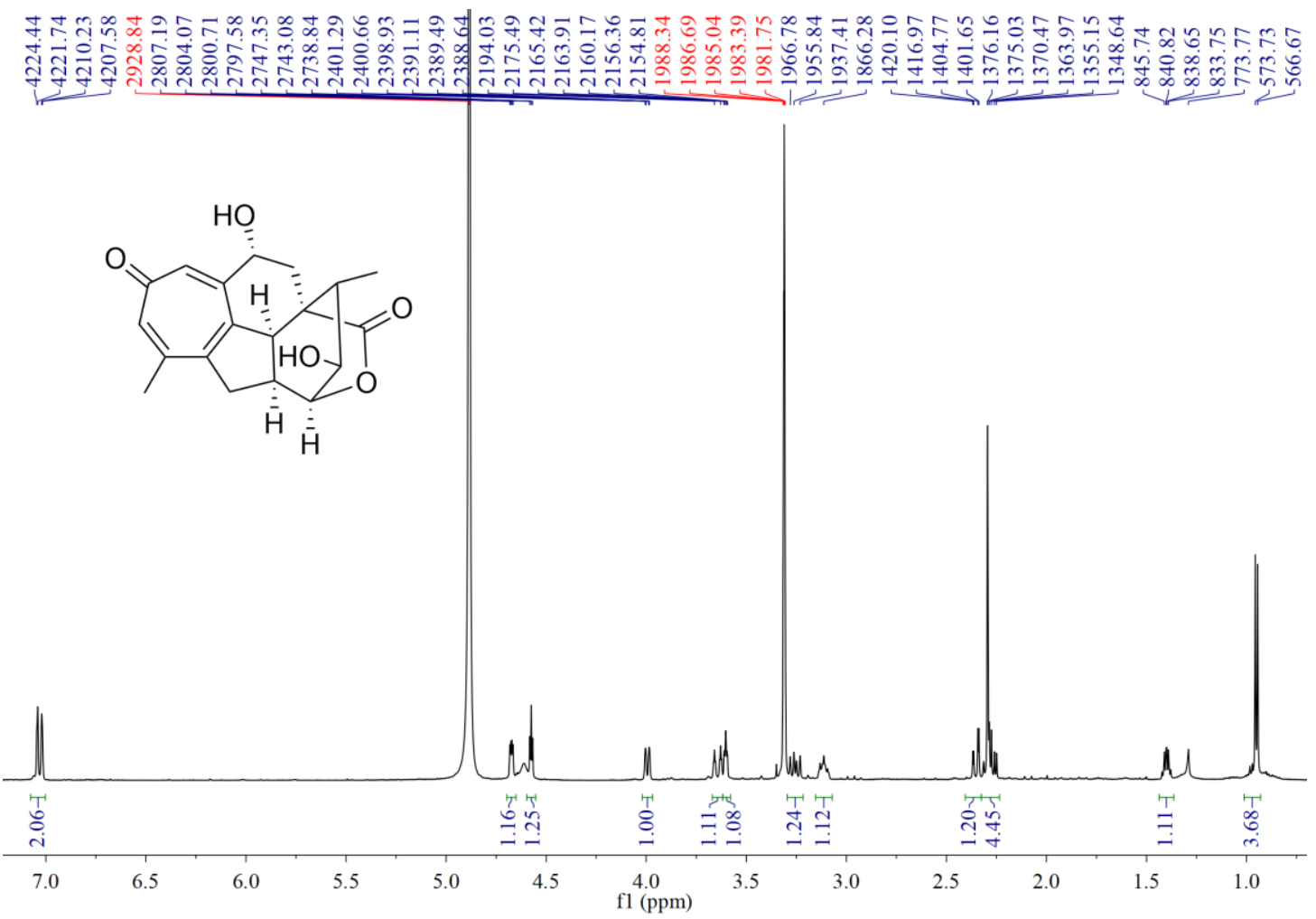


Figure S30. ${ }^{13} \mathrm{C}$ NMR spectrum of fortalpinoid B (2) in methanol- $d_{4}$.

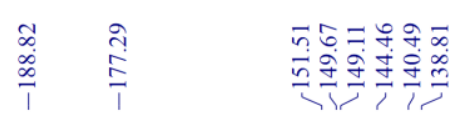

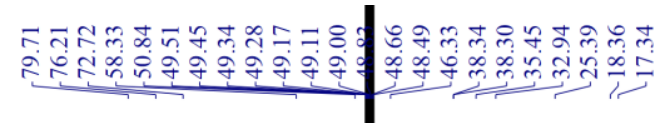

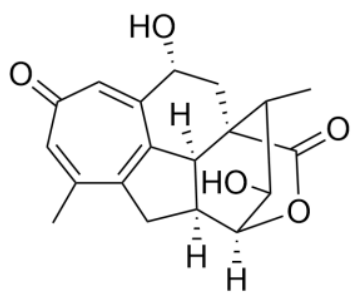
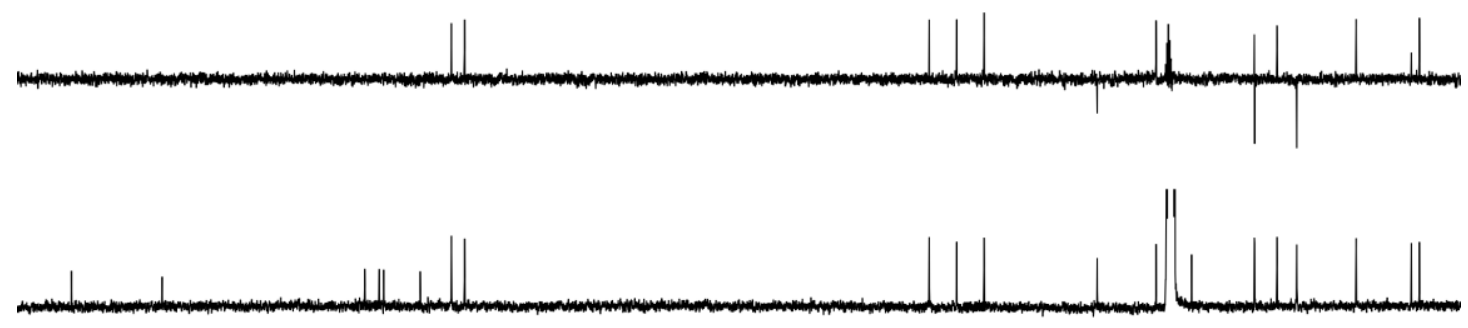

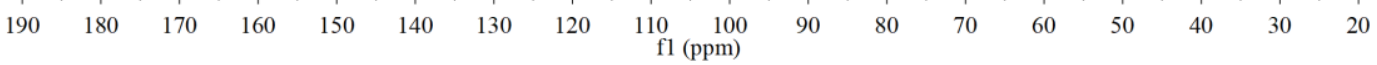

Figure S31. HSQC spectrum of fortalpinoid B (2) in pyridine- $d_{5}$.

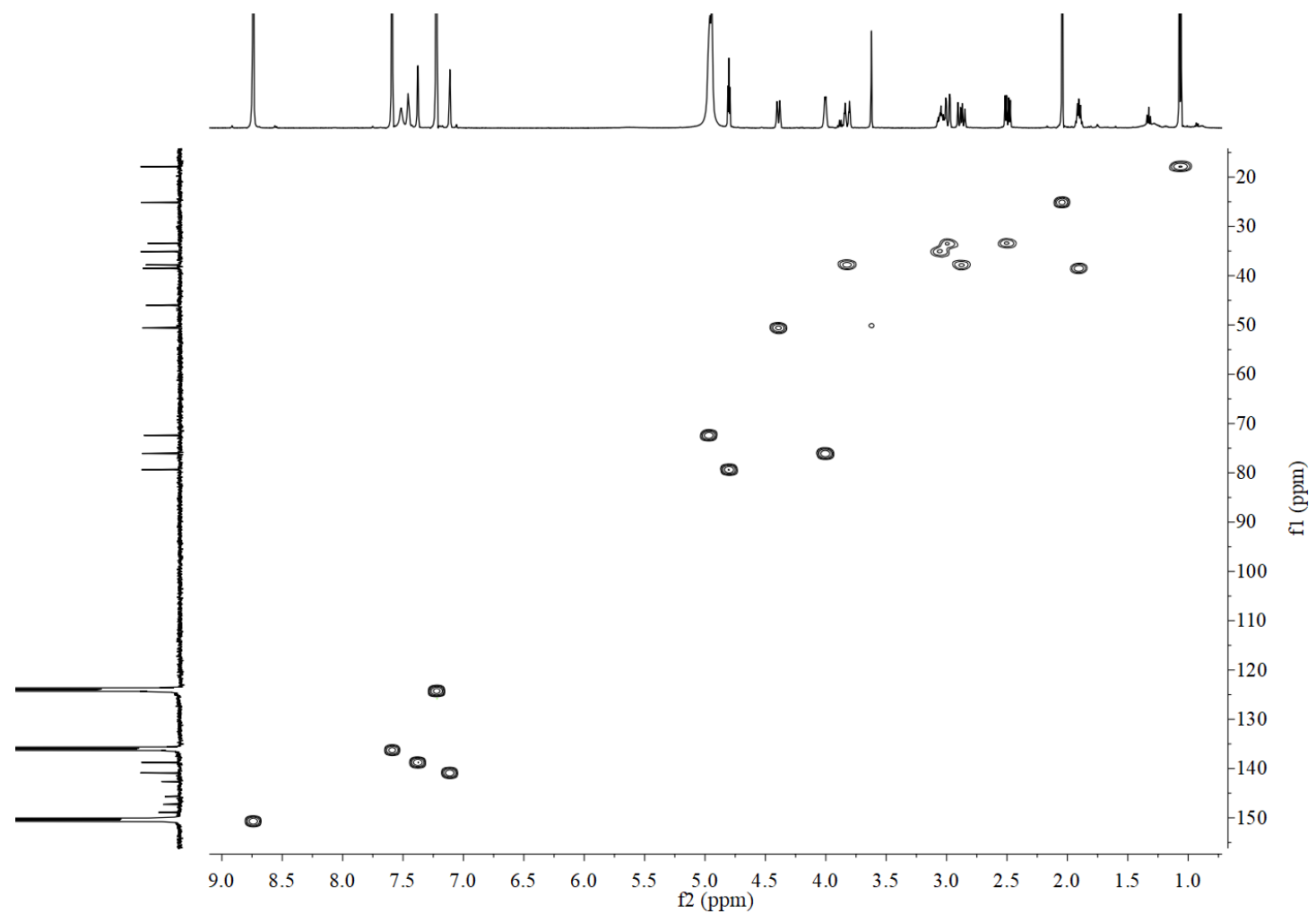


Figure S32. HMBC spectrum of fortalpinoid B (2) in pyridine- $d_{5}$

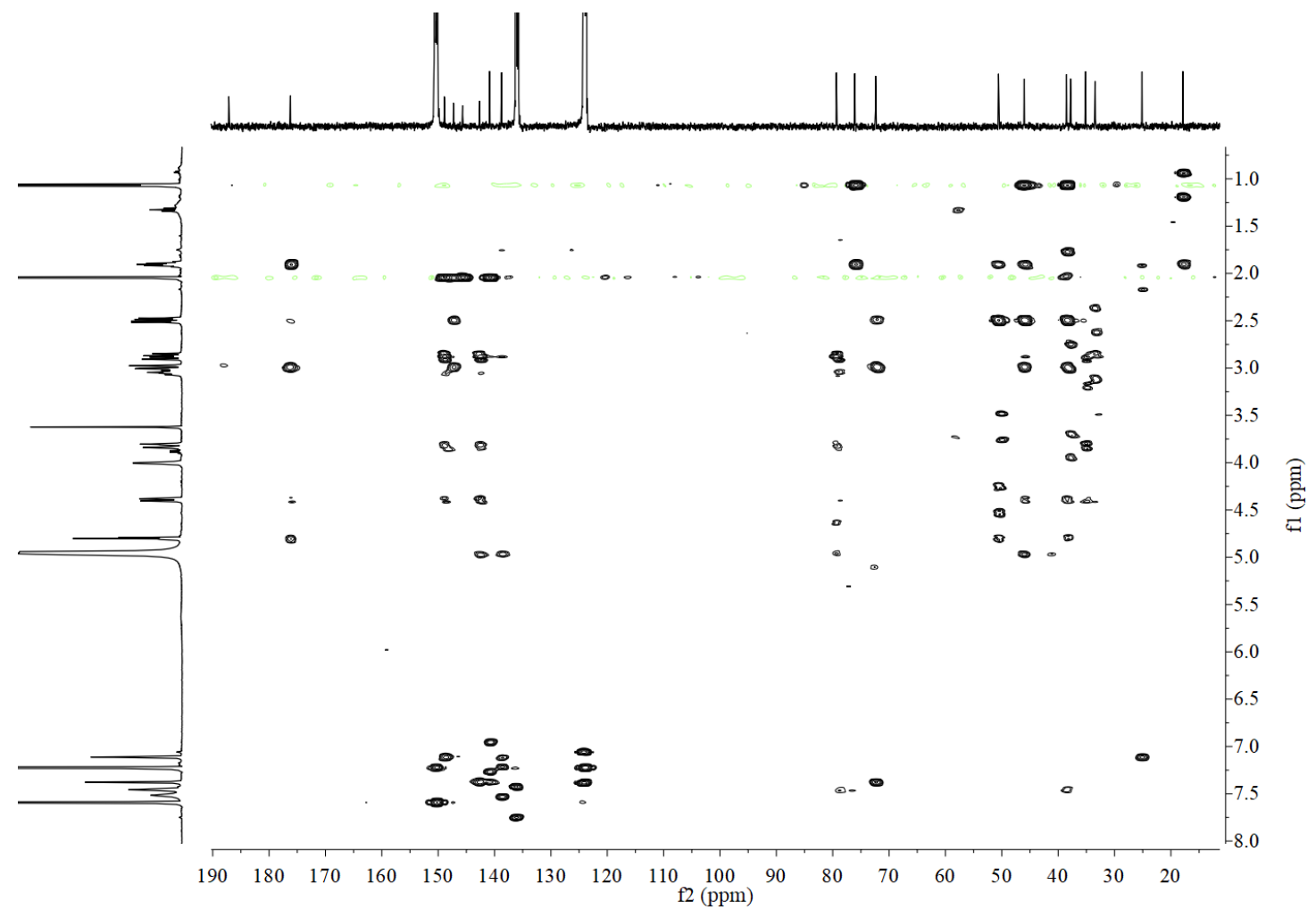

Figure S33. ${ }^{1} \mathrm{H}-{ }^{1} \mathrm{H}$ COSY spectrum of fortalpinoid B (2) in pyridine- $d_{5}$.

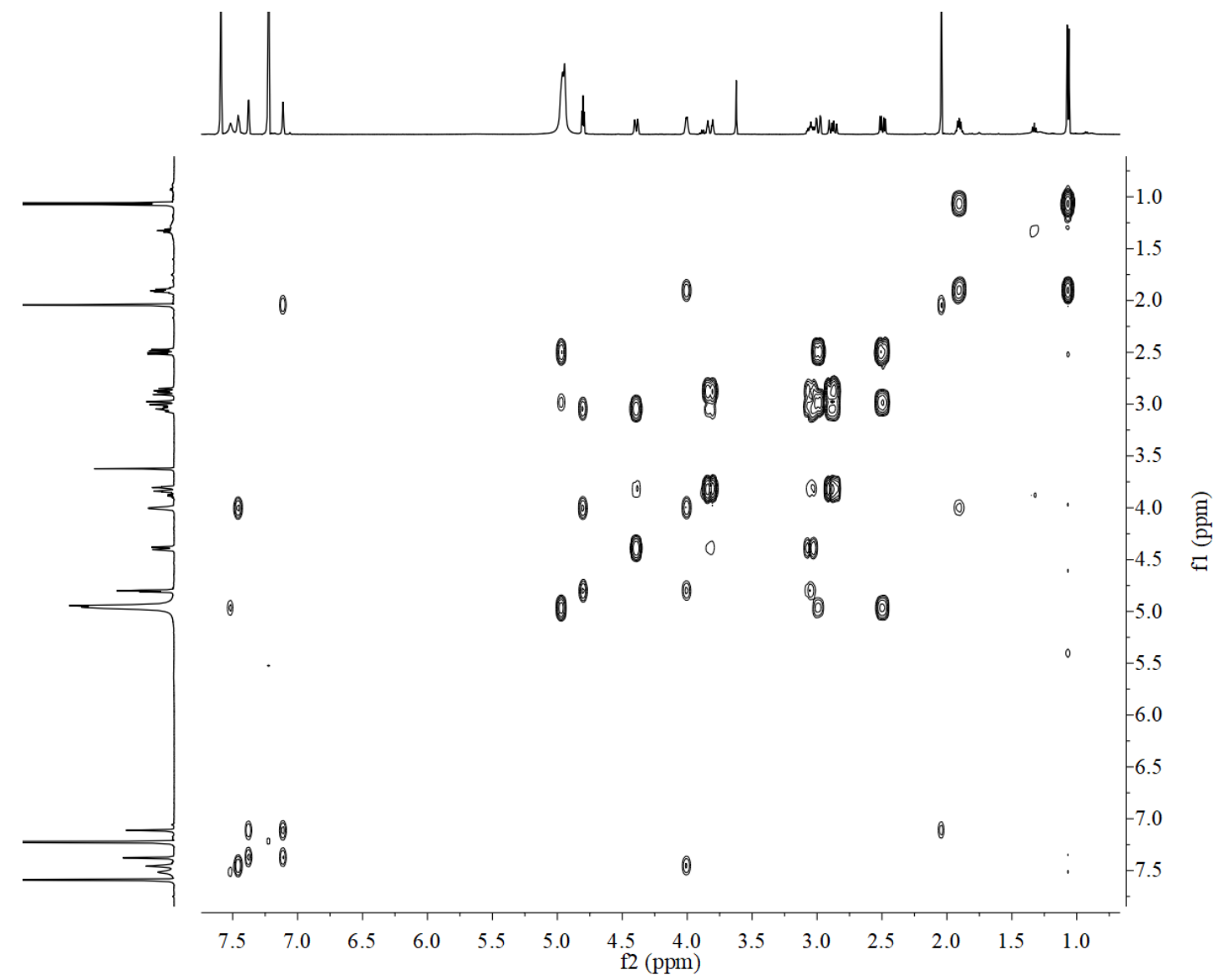


Figure S34. NOESY spectrum of fortalpinoid B (2) in pyridine- $d_{5}$

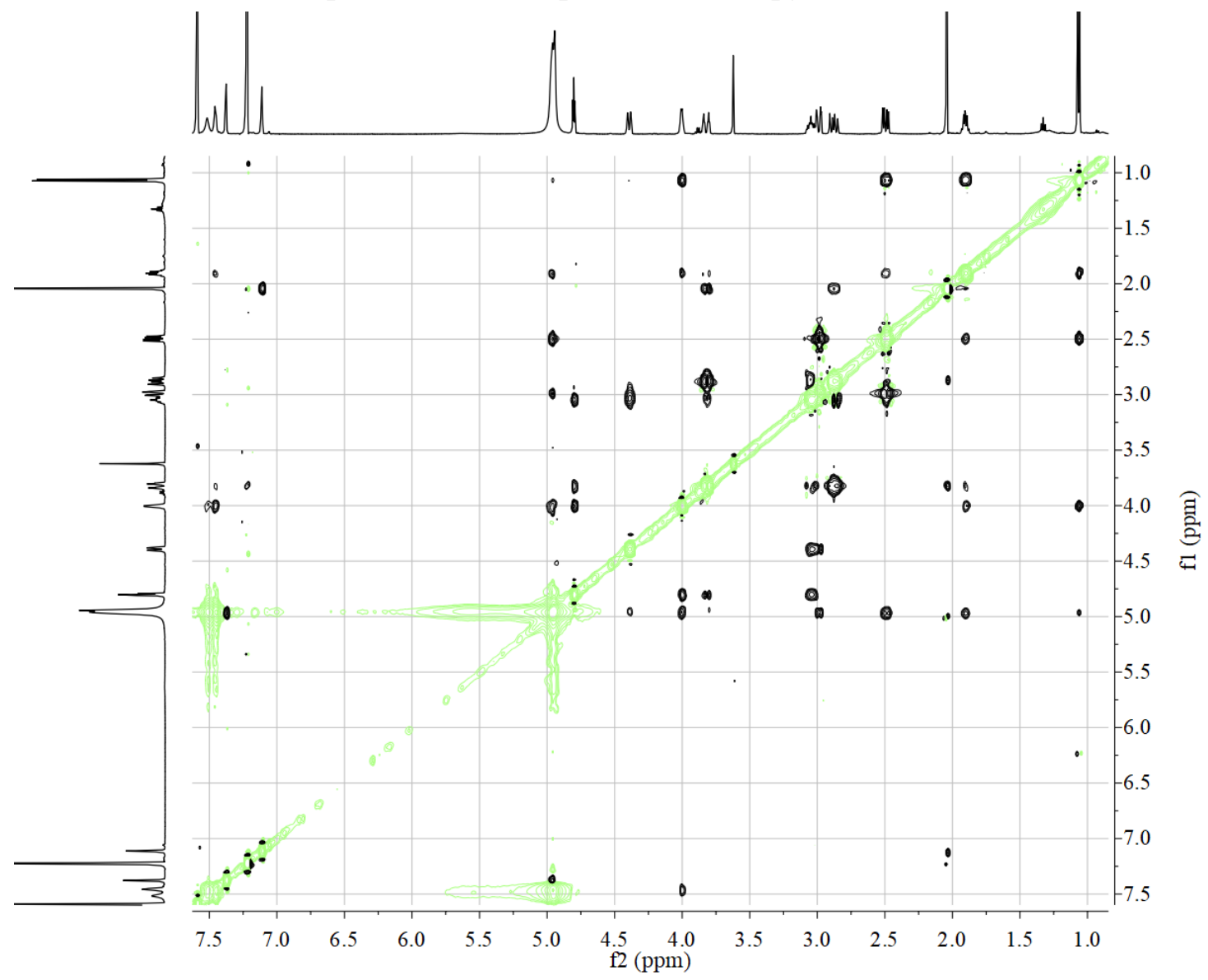

Figure S35. NOESY spectrum of fortalpinoid B (2) in methanol- $d_{4}$.

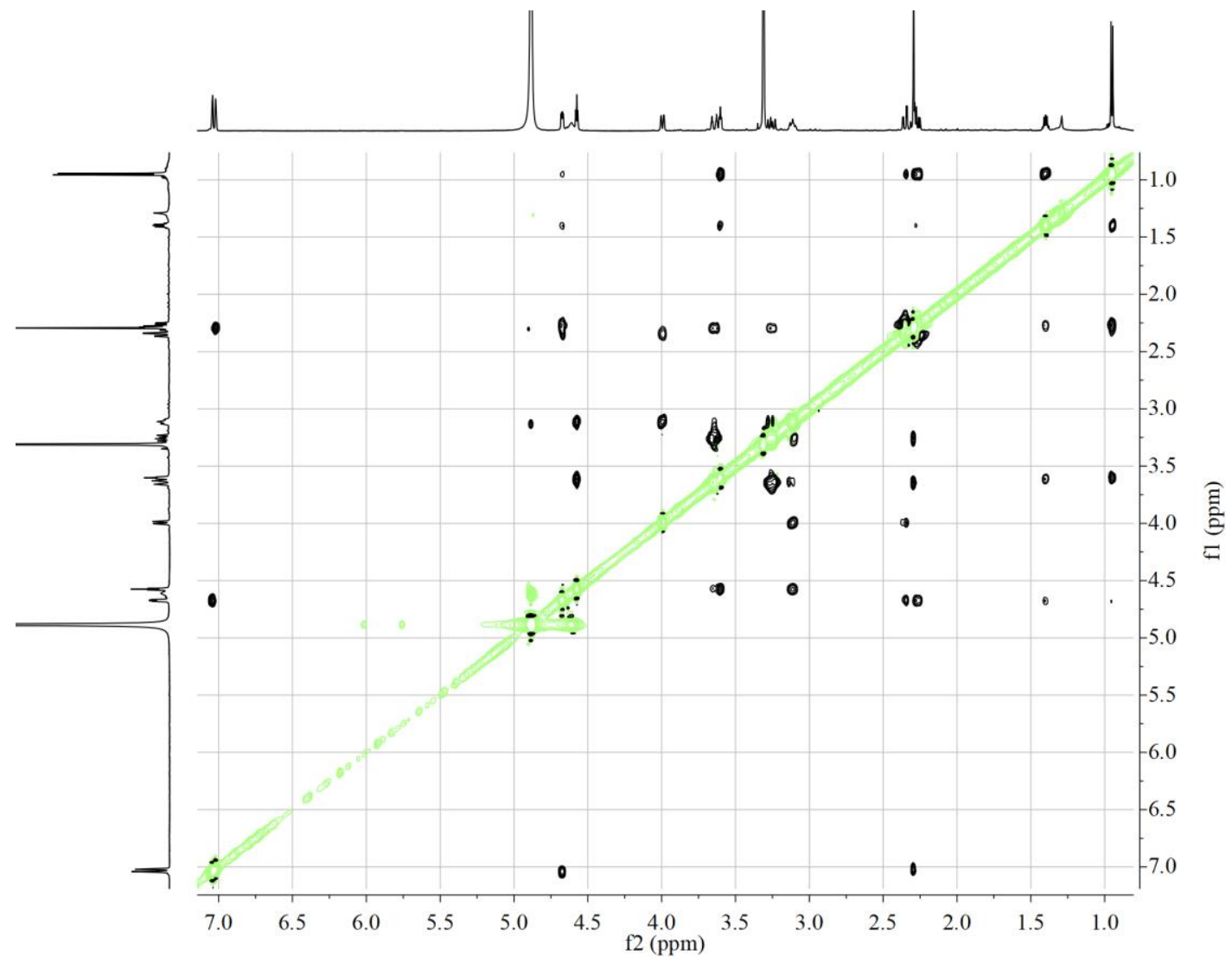


Figure S36. (+)-ESIMS spectrum of fortalpinoid B (2).

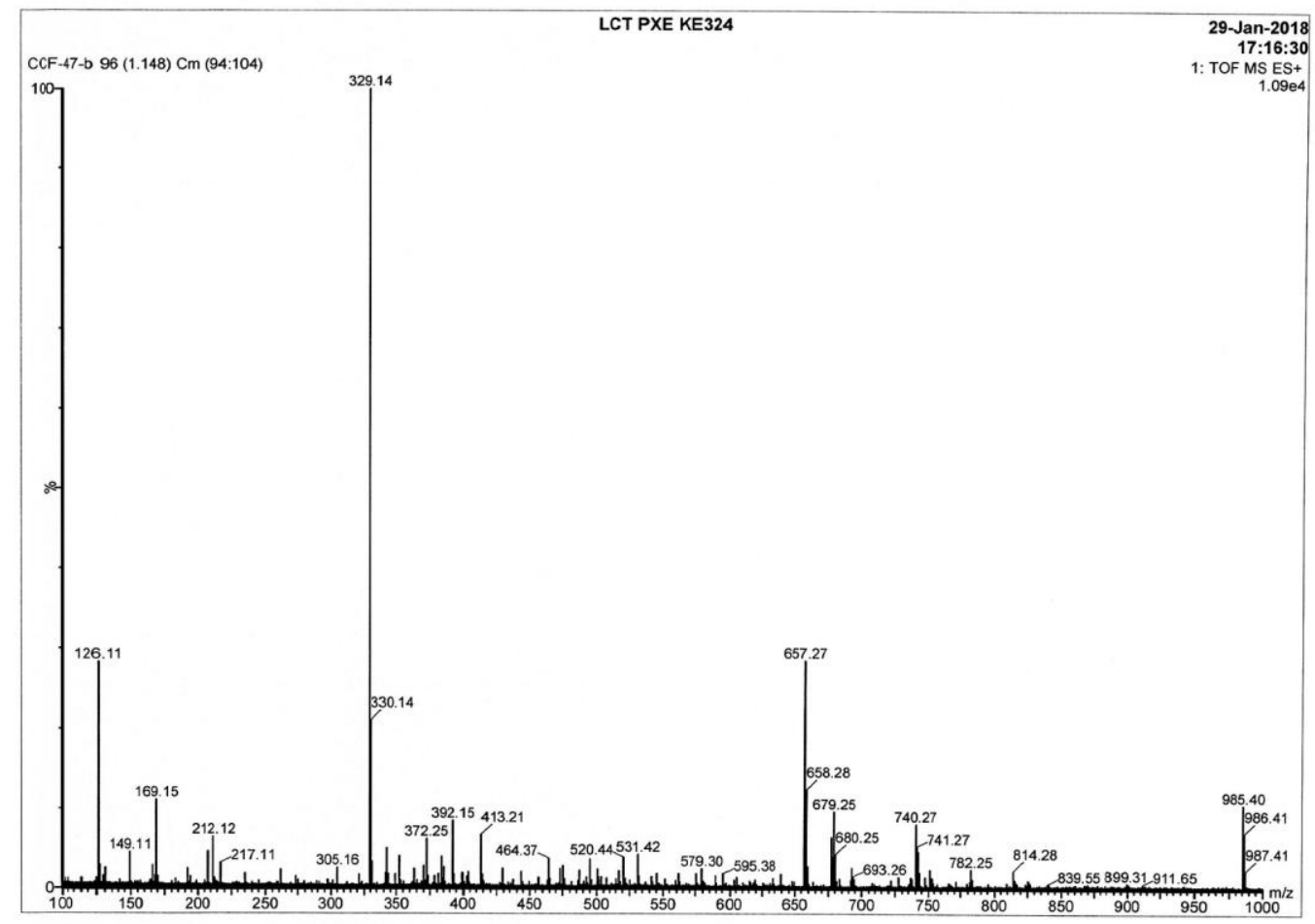

Figure S37. (-)-ESIMS spectrum of fortalpinoid B (2).

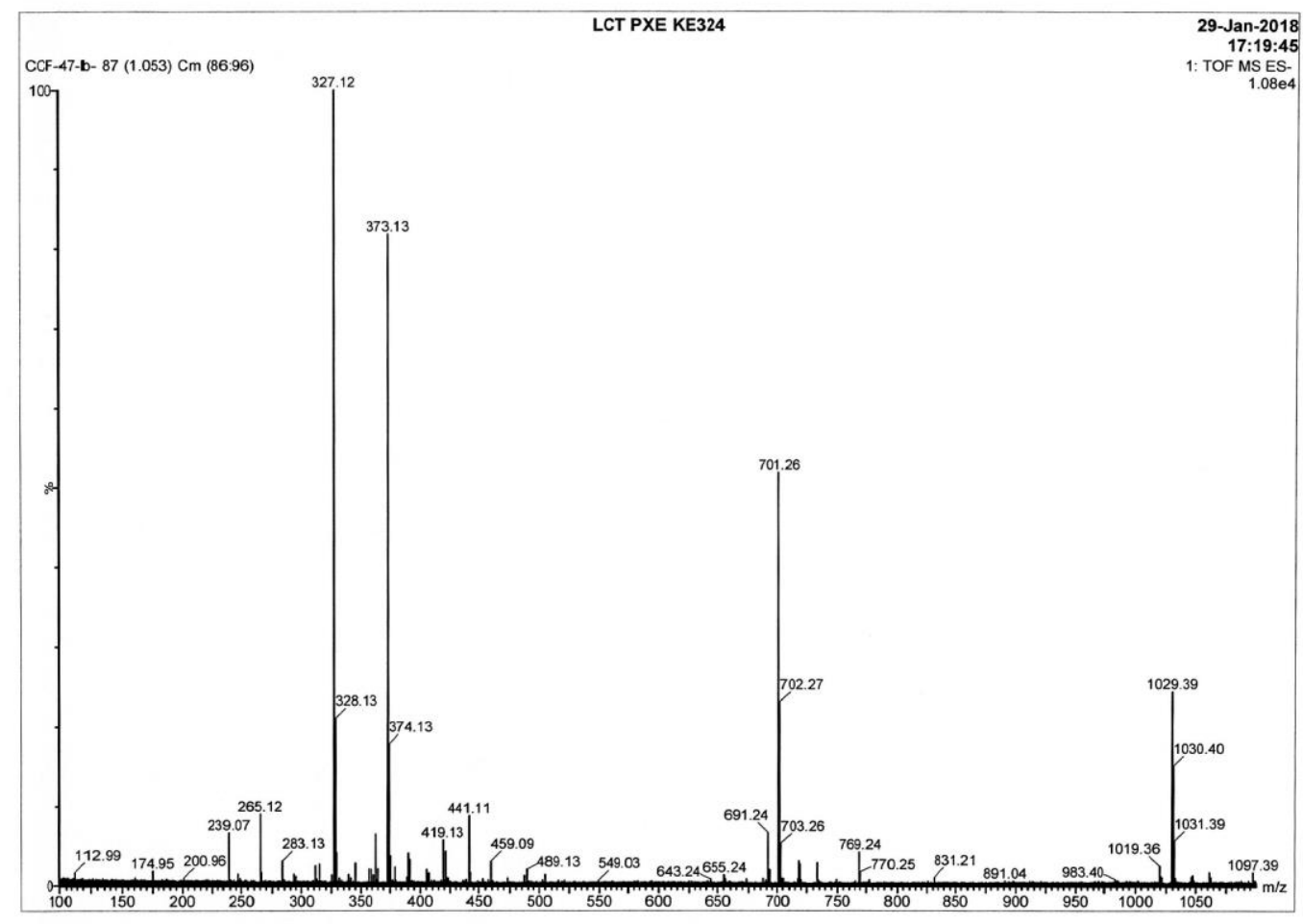


Figure S38. (+)-HRESIMS spectrum of fortalpinoid B (2).

Elemental Composition Report

Page 1

Single Mass Analysis

Tolerance $=3.0$ PPM I DBE: $\min =-1.5, \max =50.0$

Element prediction: Off

Number of isotope peaks used for i-FIT $=3$

Monoisotopic Mass, Even Electron lons

115 formula(e) evaluated with 1 results within limits (up to 50 best isotopic matches for each mass)

Elements Used:

$\begin{array}{llll}\text { C: } 0-100 & \text { H: } 0-1000 & \text { O: } 0-500 & \mathrm{Na}: 0-1\end{array}$

LCT PXE KE324

29-Jan-2018

CCF-47-b 159 (1.877) Cm (14:16:30

1: TOF MS ES+

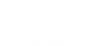

$2.57 \mathrm{e}+004$

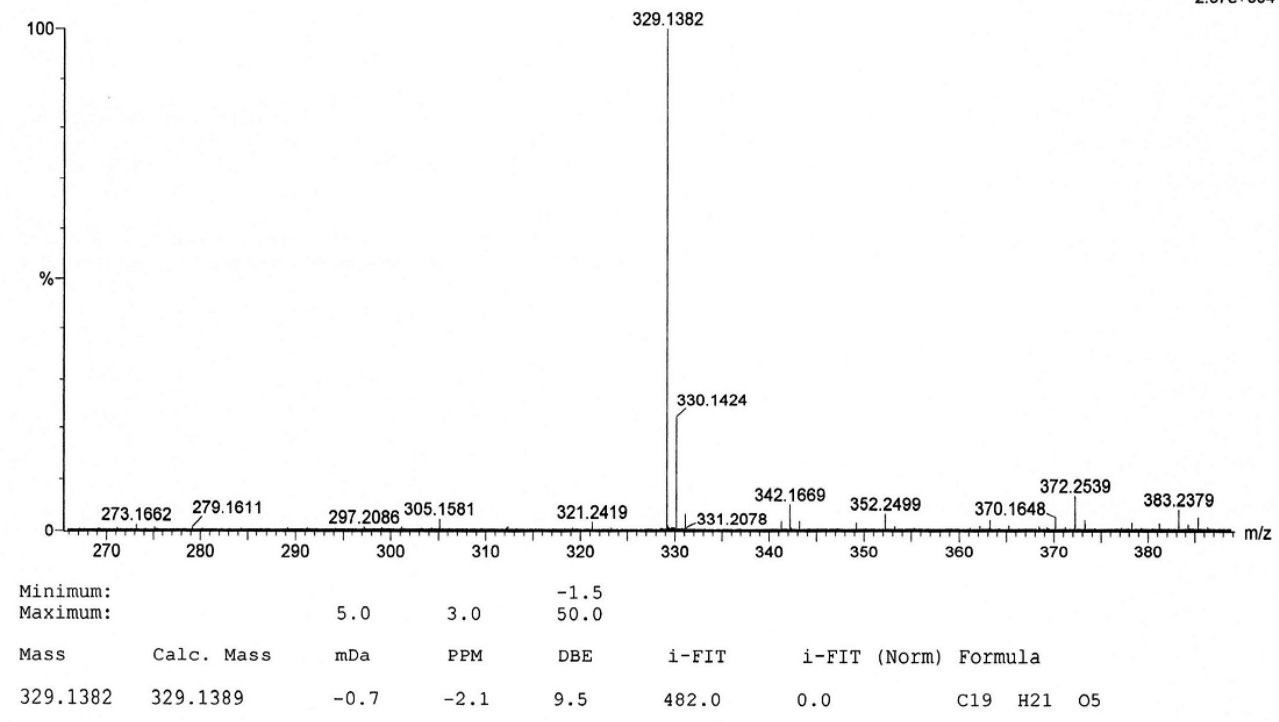

Figure S39. IR spectrum of fortalpinoid B (2).

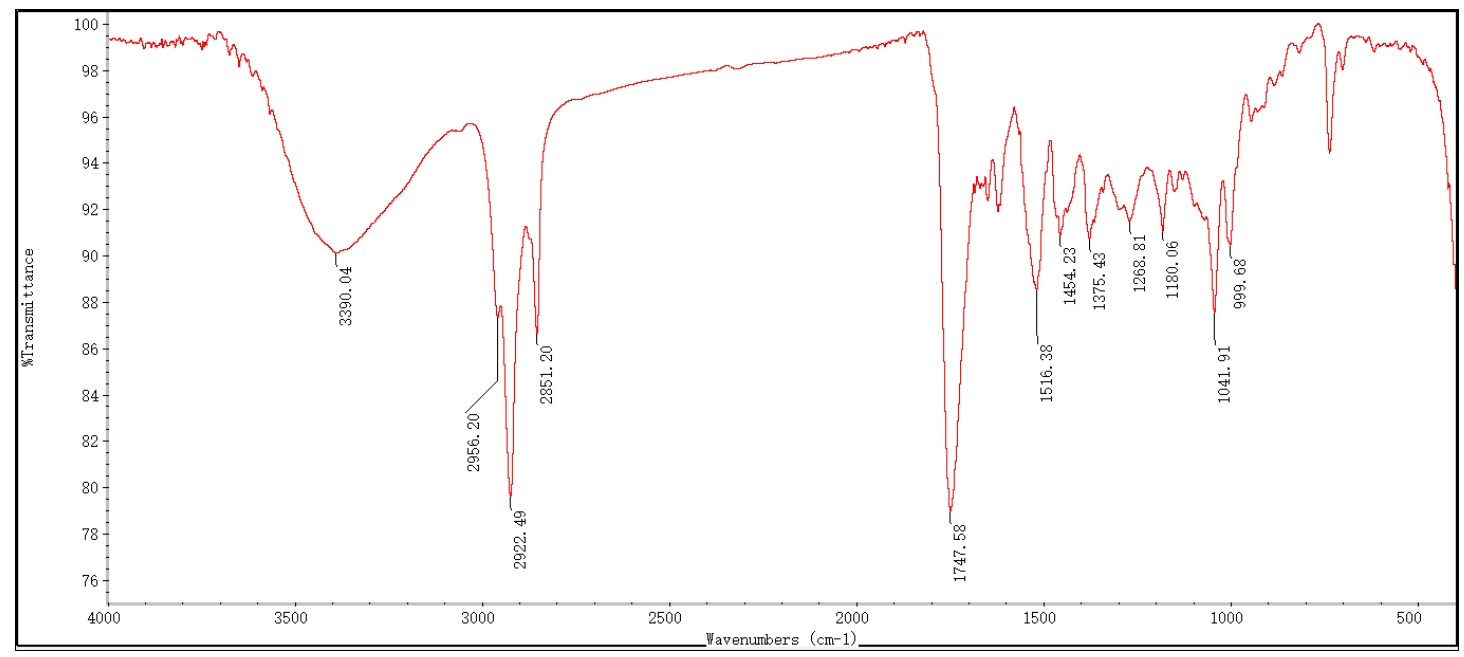


Figure S40. ${ }^{1} \mathrm{H}$ NMR spectrum of fortalpinoid C (3) in pyridine- $d_{5}$.

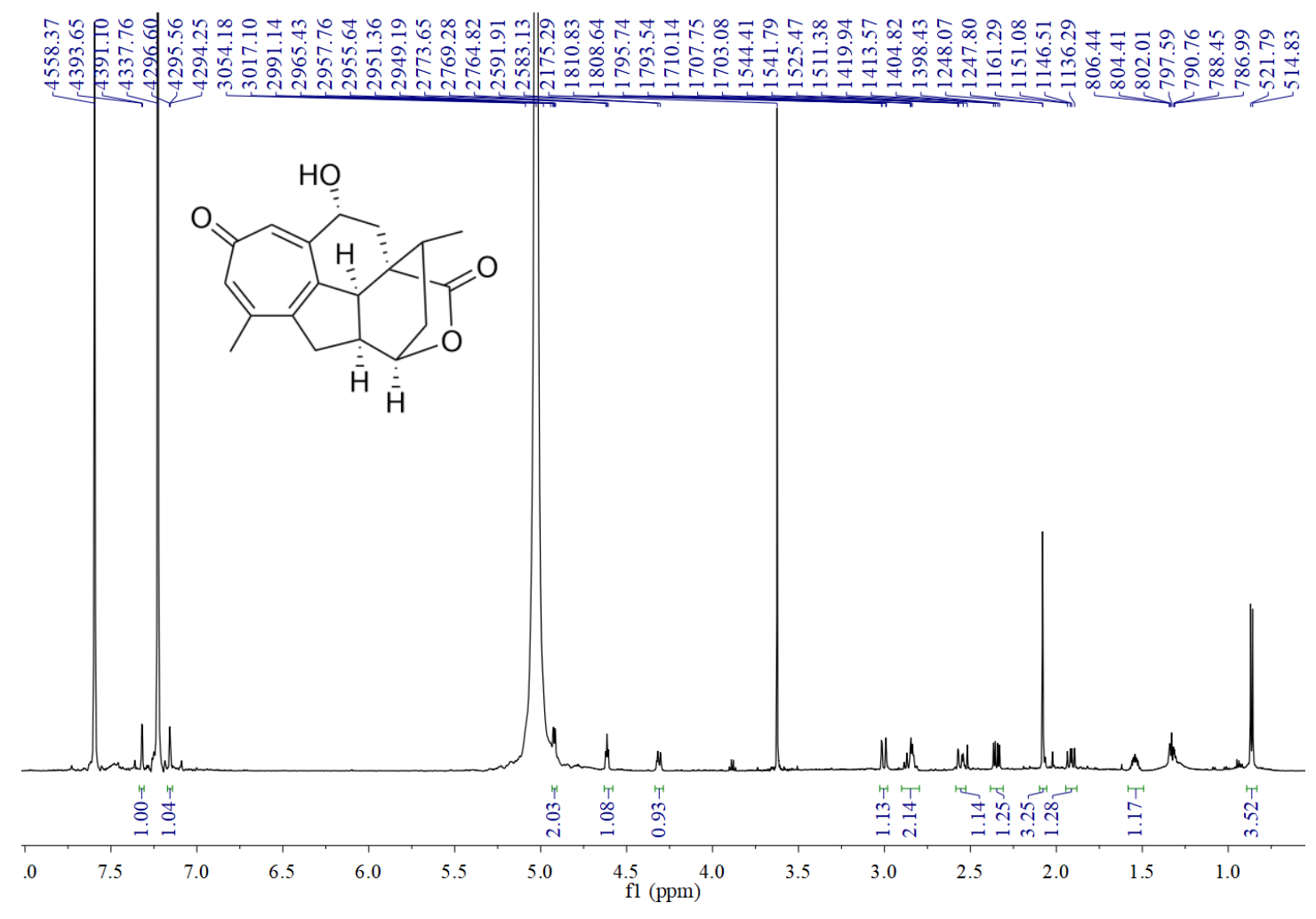

Figure S41. ${ }^{13} \mathrm{C}$ NMR spectrum of fortalpinoid C (3) in pyridine- $d_{5}$.

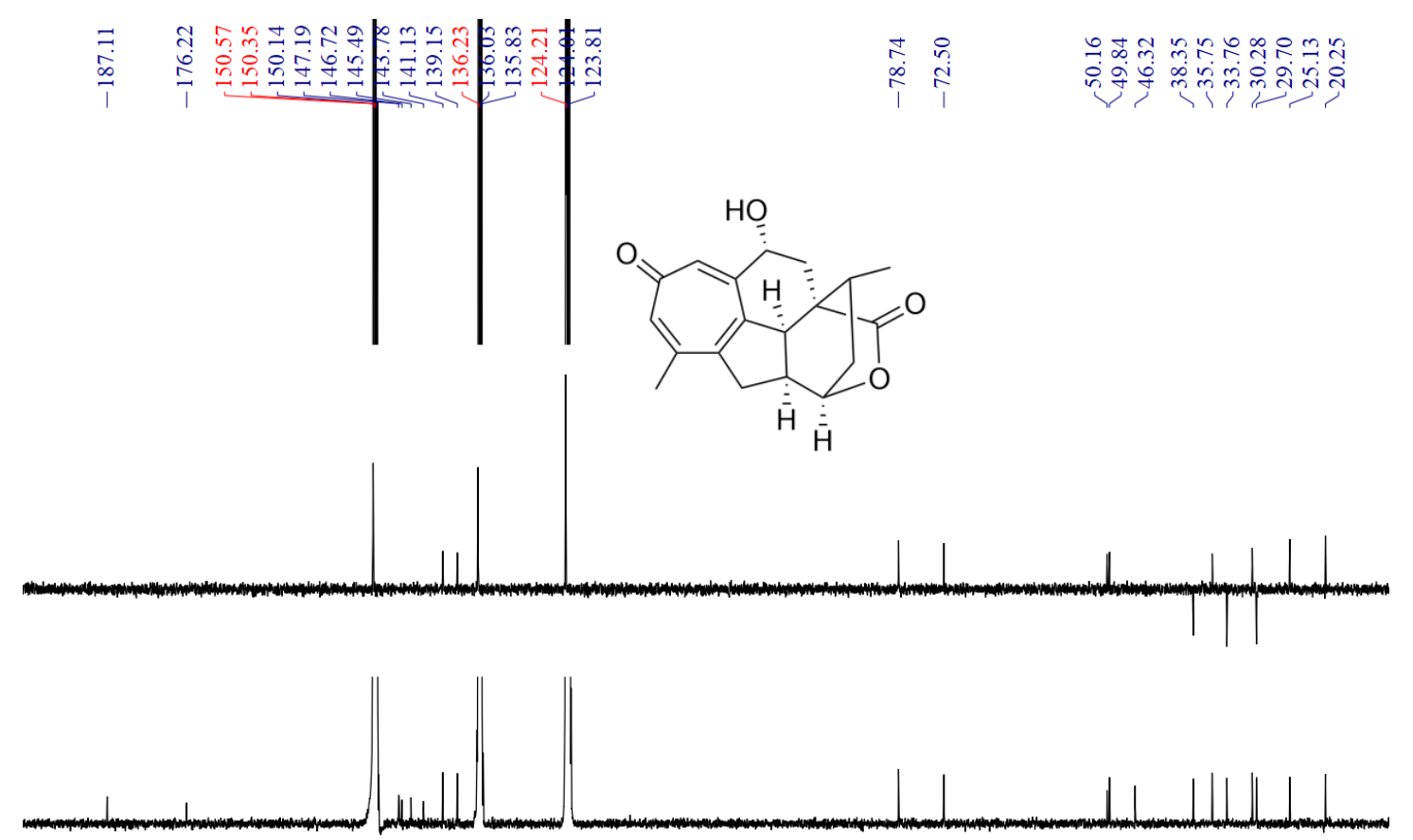

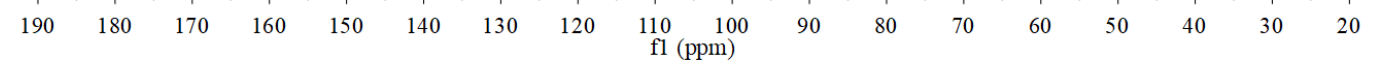


Figure S42. HSQC spectrum of fortalpinoid C (3) in pyridine- $d_{5}$.

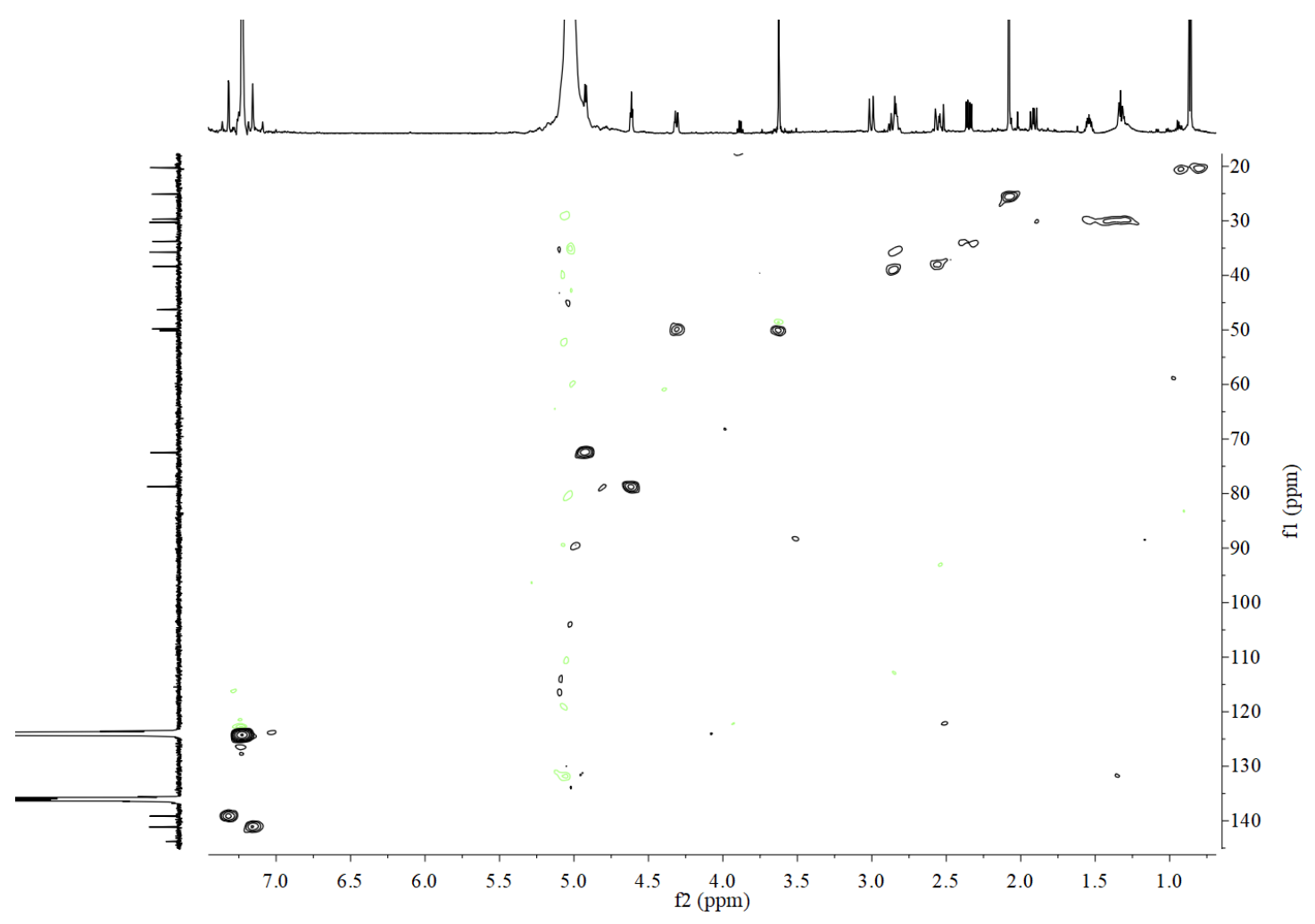

Figure S43. HMBC spectrum of fortalpinoid C (3) in pyridine- $d 5$.

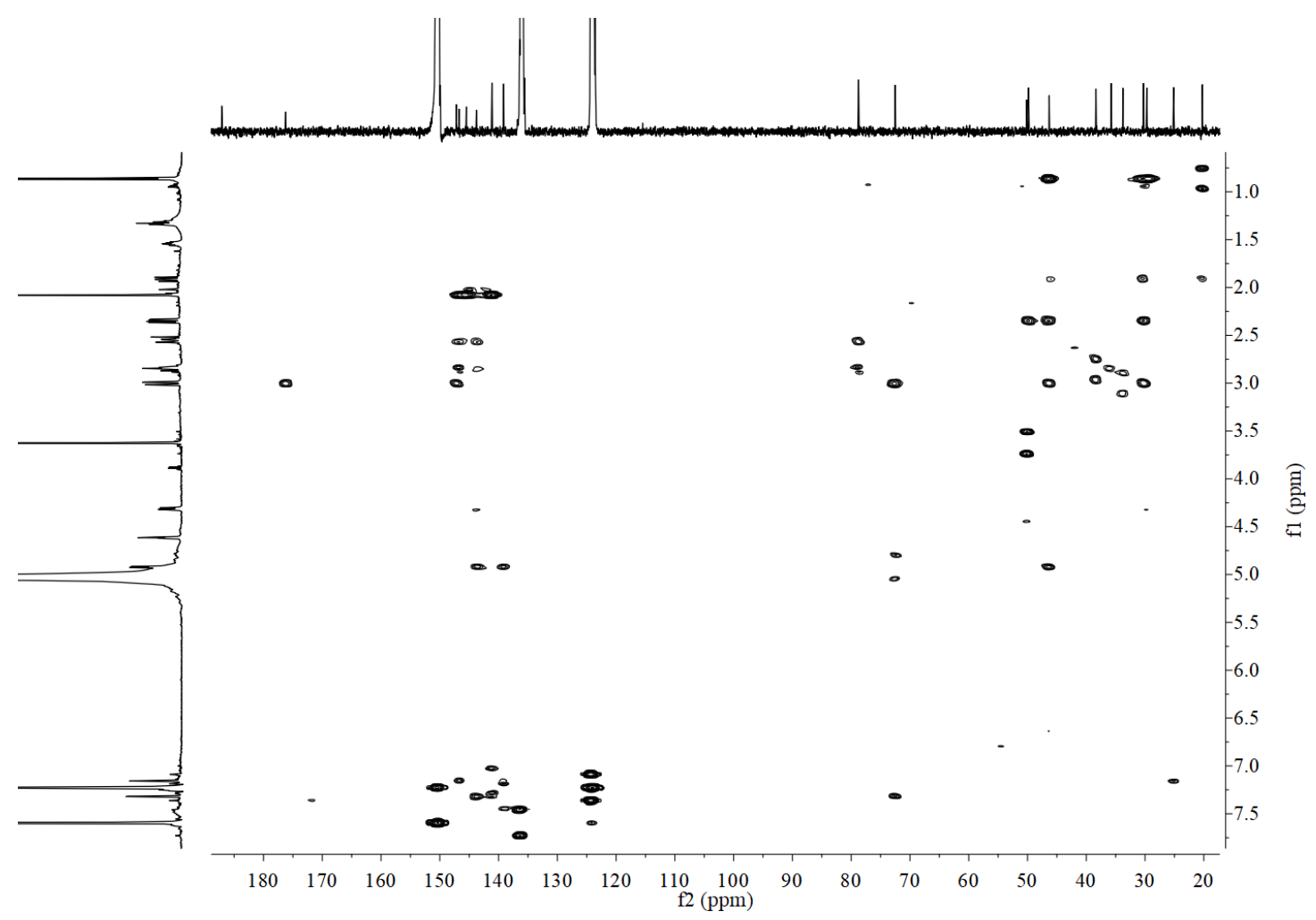


Figure S44. ${ }^{1} \mathrm{H}-{ }^{1} \mathrm{H}$ COSY spectrum of fortalpinoid C (3) in pyridine- $d 5$.

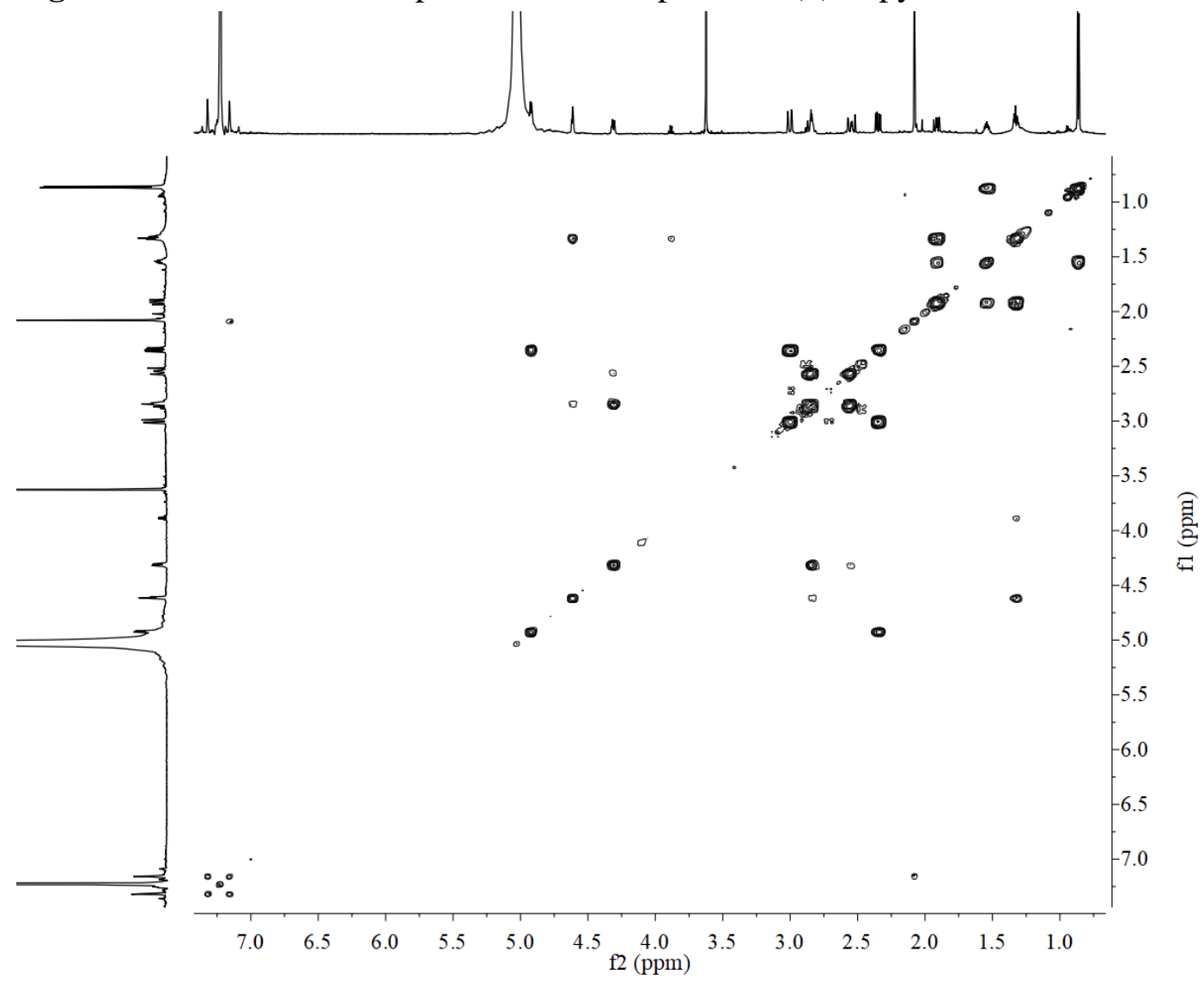

Figure S45. NOESY spectrum of fortalpinoid C (3) in pyridine- $d_{5}$.

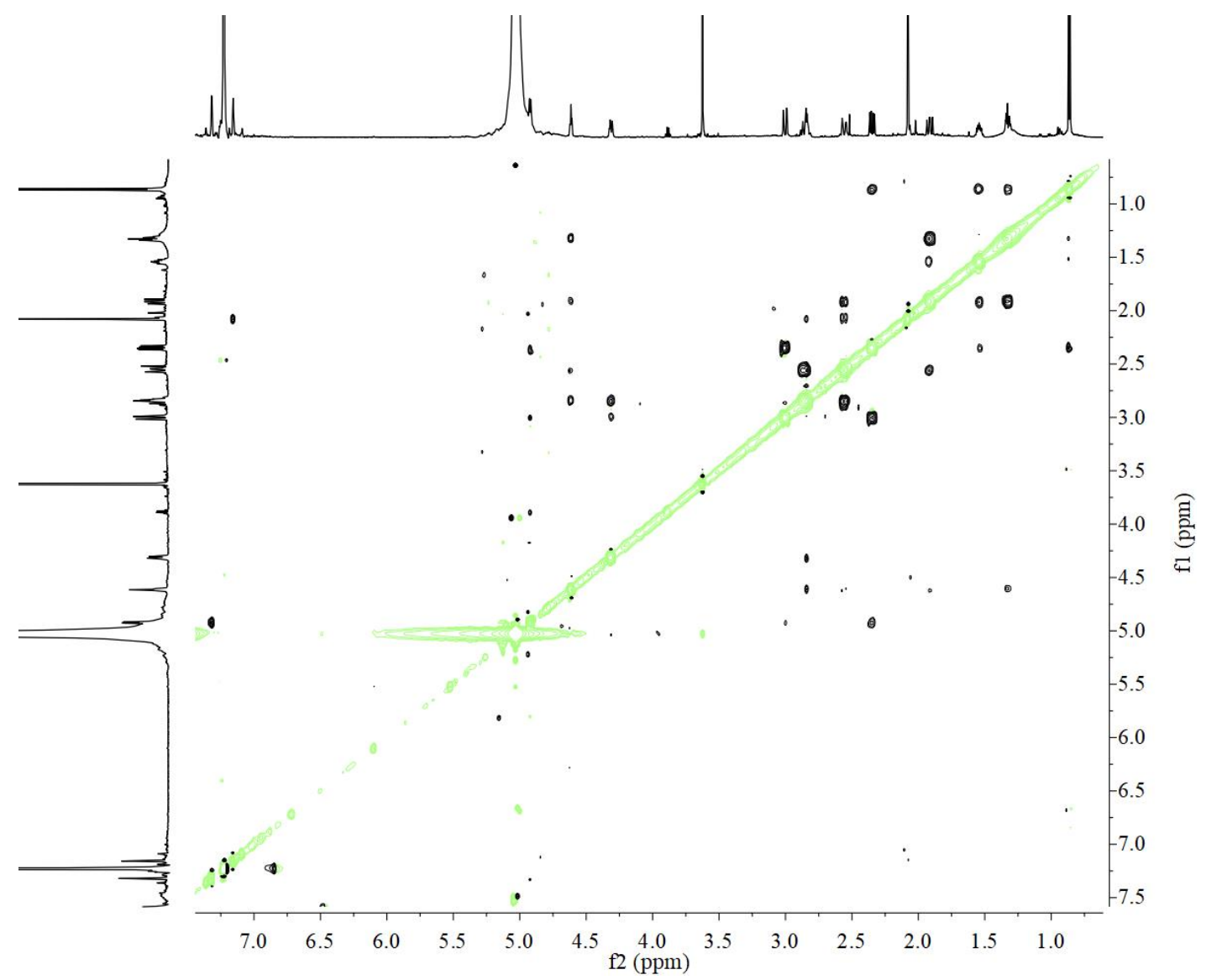


Figure S46. (+)-ESIMS spectrum of fortalpinoid C (3).

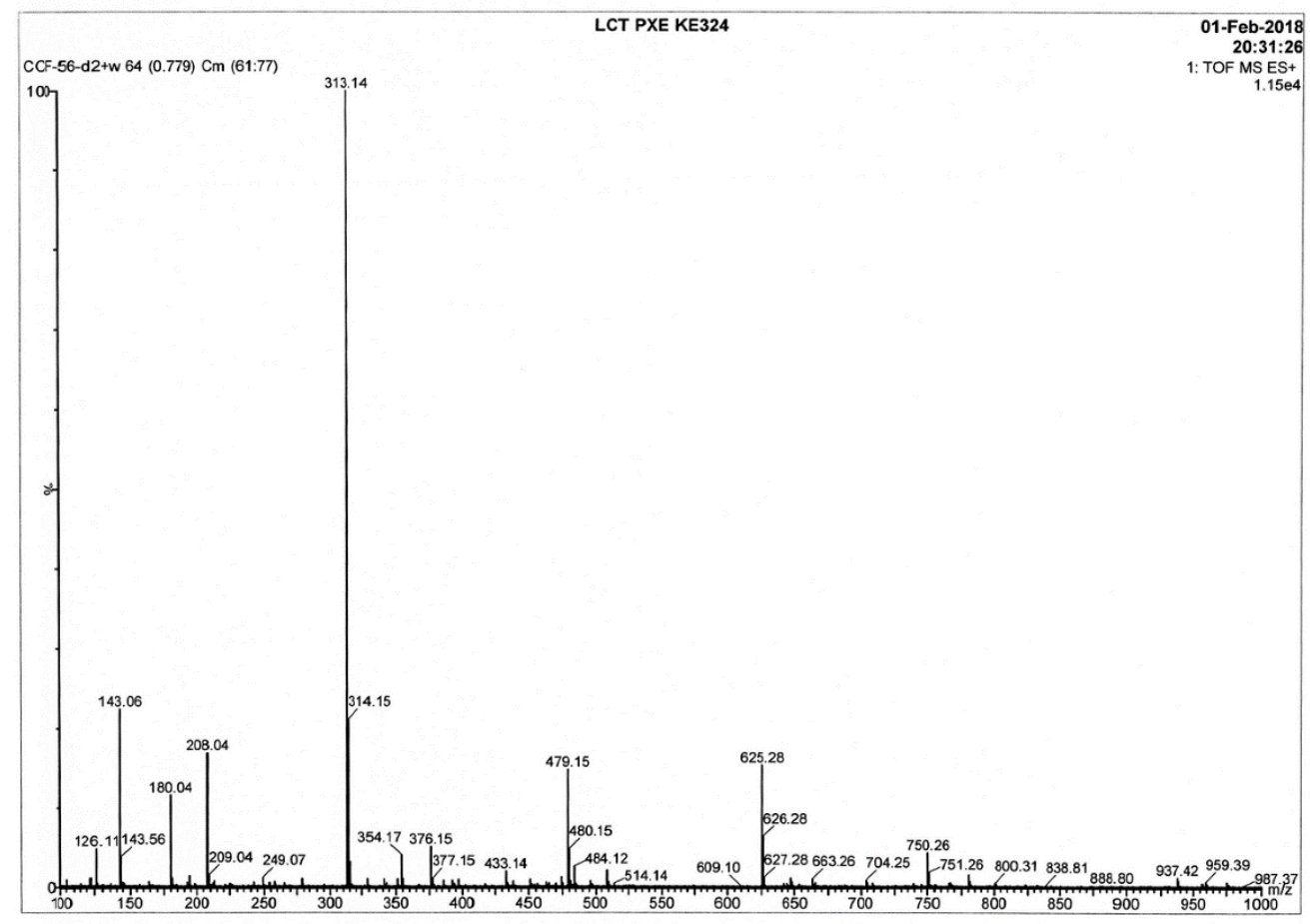

Figure S47. (-)-ESIMS spectrum of fortalpinoid C (3).

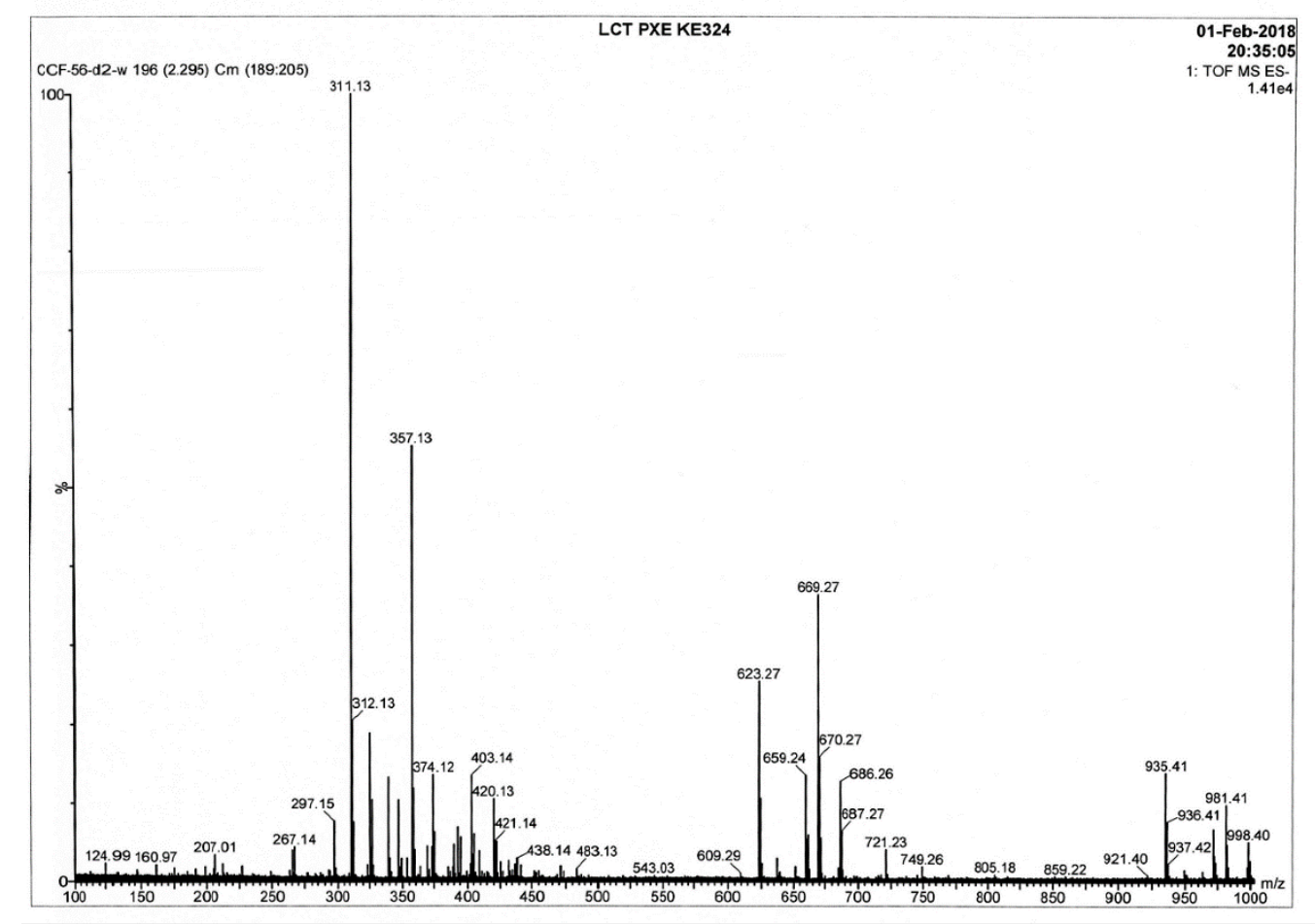


Figure S48. (+)-HRESIMS spectrum of fortalpinoid C (3).

Elemental Composition Report

Page 1

Single Mass Analysis

Tolerance $=3.0$ PPM I DBE: $\min =-1.5, \max =50.0$

Element prediction: Off

Number of isotope peaks used for $\mathrm{i}-\mathrm{FIT}=3$

Monoisotopic Mass, Even Electron Ions

106 formula(e) evaluated with 1 results within limits (up to 50 best isotopic matches for each mass)

Elements Used:

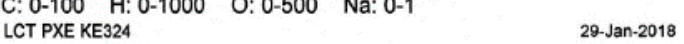

$21: 15: 14$
$(101: 104)$

1: TOF MS ES+

313.1443

$2.15 \mathrm{e}+003$

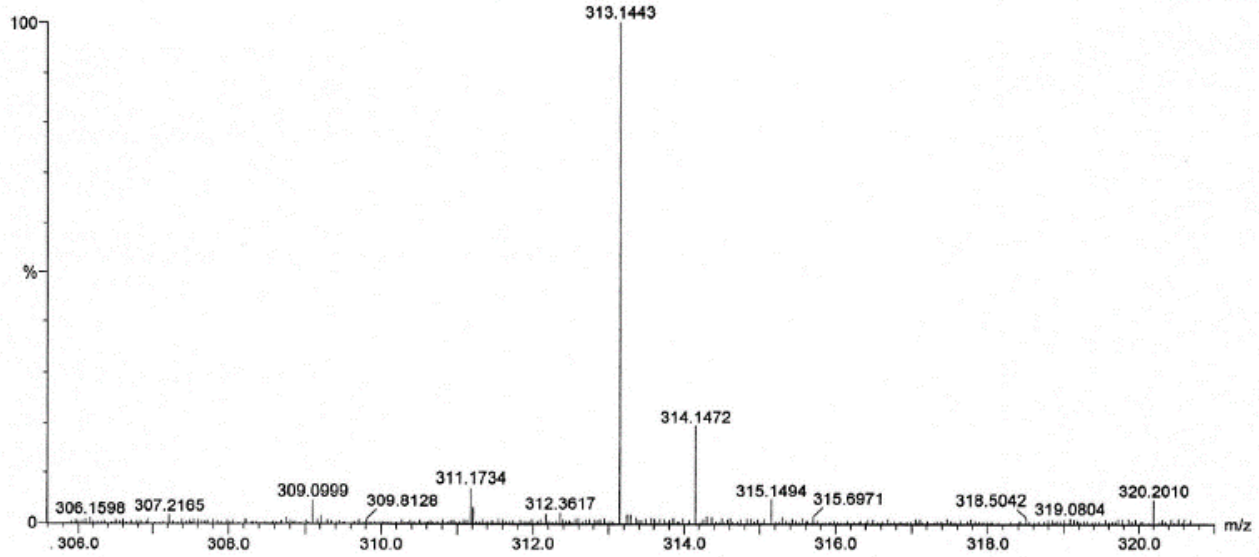

Figure S49. IR spectrum of fortalpinoid C (3).

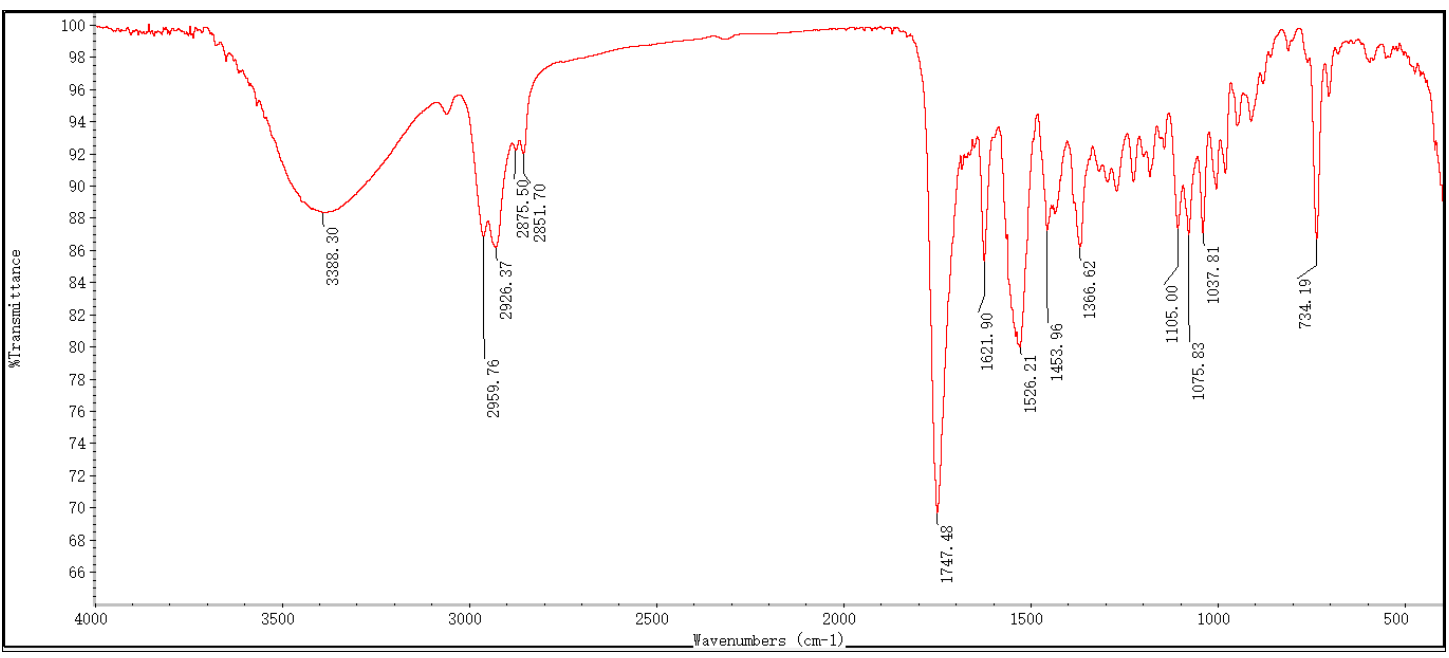


Figure S50. ${ }^{1} \mathrm{H}$ NMR spectrum of fortalpinoid D (4) in $\mathrm{CDCl}_{3}$.

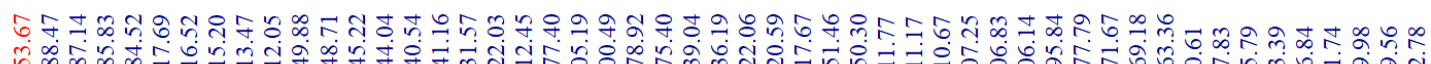

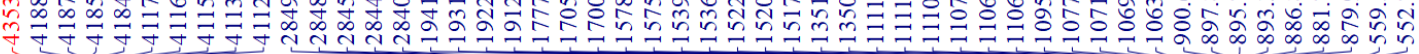

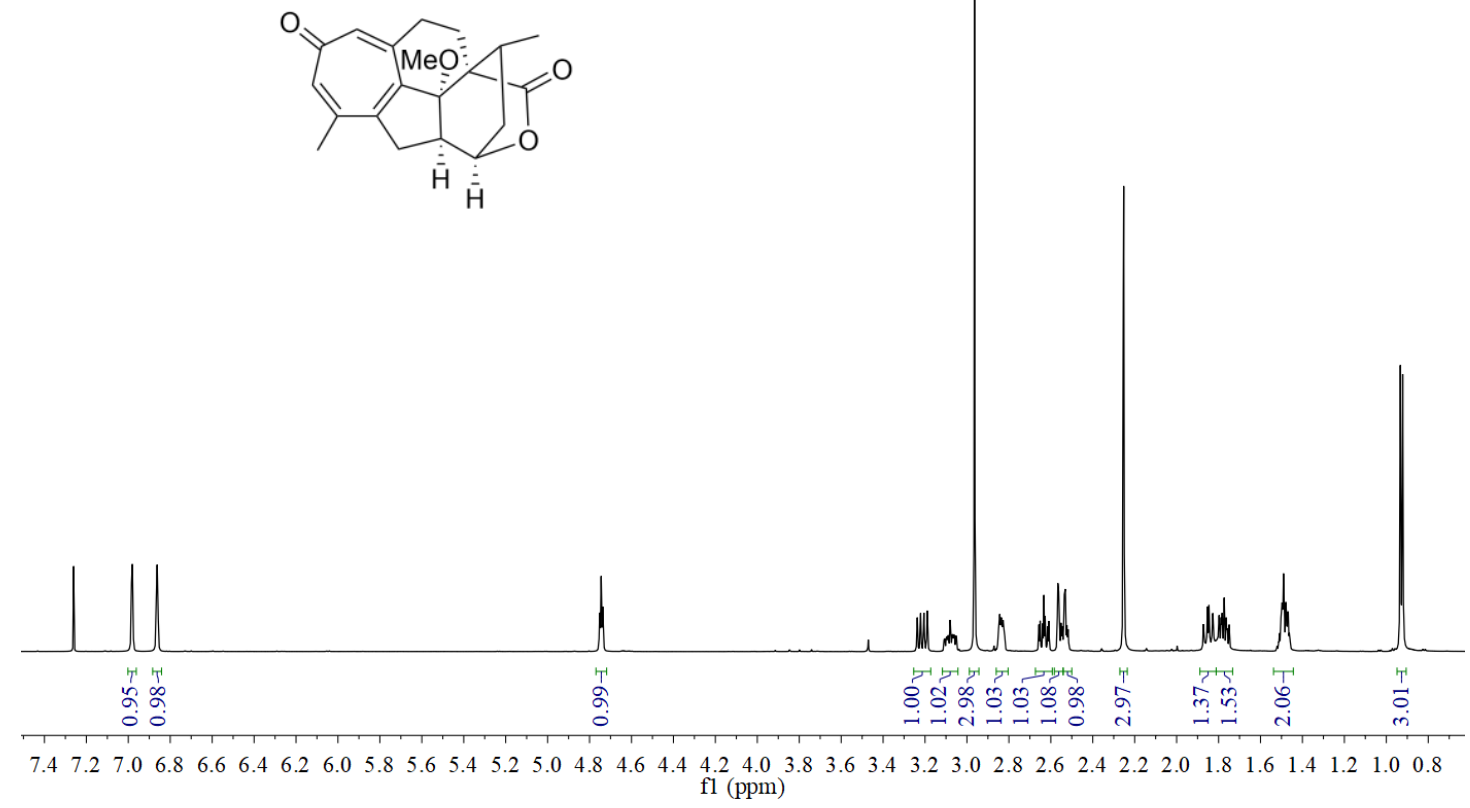

Figure S51. ${ }^{13} \mathrm{C}$ NMR spectrum of fortalpinoid D (4) in $\mathrm{CDCl}_{3}$.
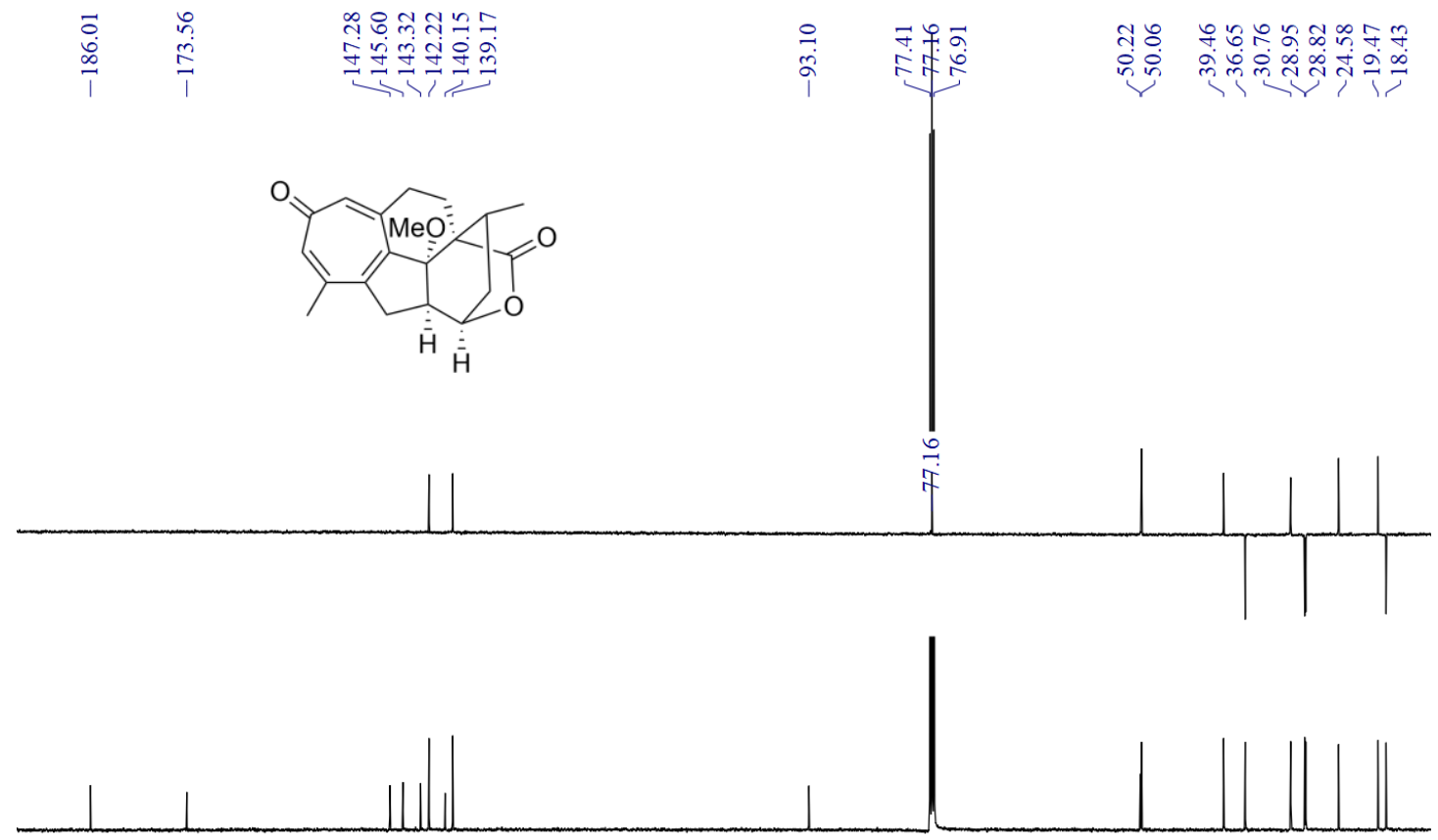

$\begin{array}{llllllllllllllll}190 & 180 & 170 & 160 & 150 & 140 & 130 & 120 & \begin{array}{c}110 \\ \mathrm{fl}(\mathrm{ppm})\end{array} & \begin{array}{l}100 \\ (100\end{array} & 70 & 60 & 50 & 40 & 30 & 20\end{array}$ 
Figure S52. HSQC spectrum of fortalpinoid D (4) in $\mathrm{CDCl}_{3}$.

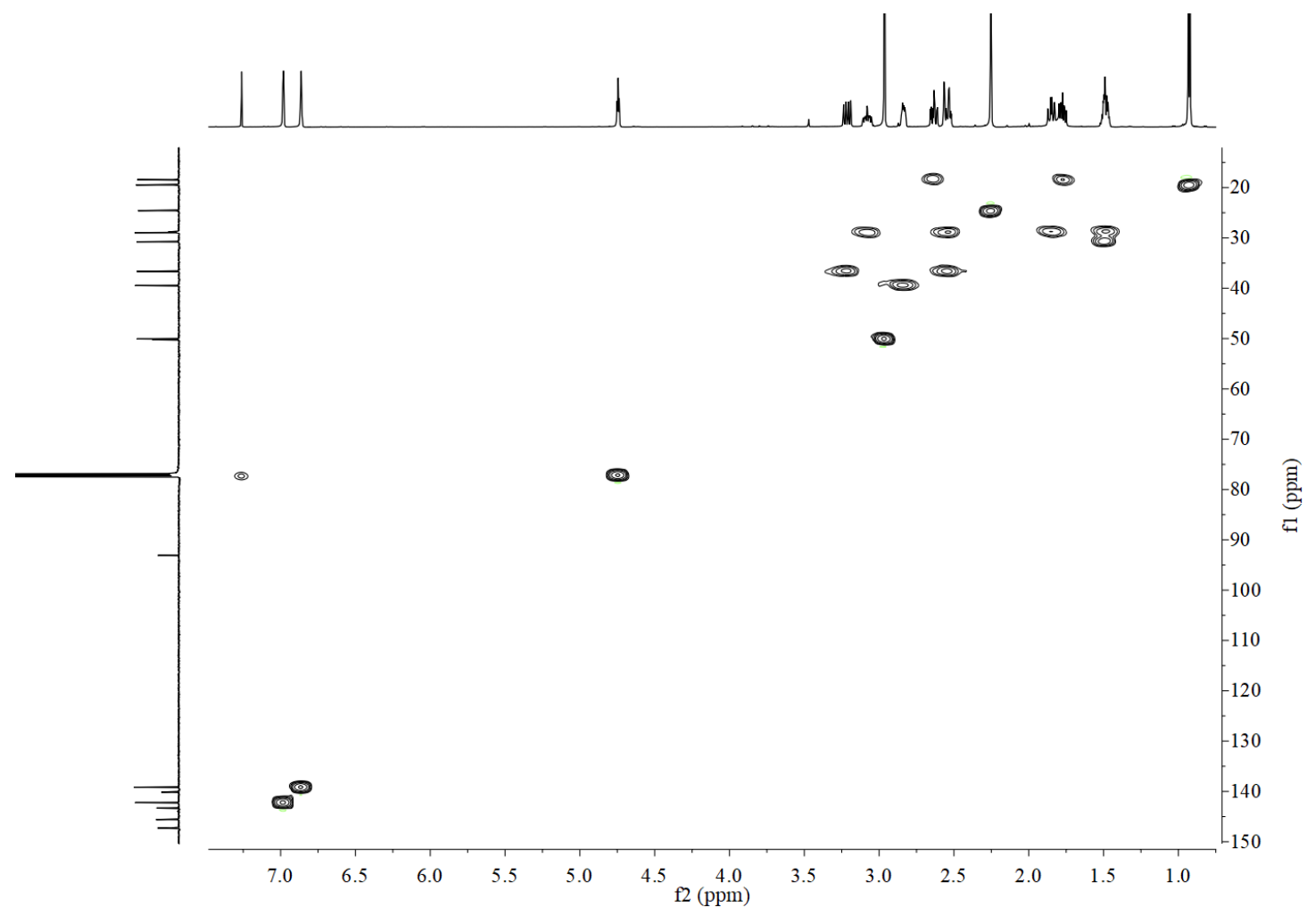

Figure S53. HMBC spectrum of fortalpinoid D (4) in $\mathrm{CDCl}_{3}$.

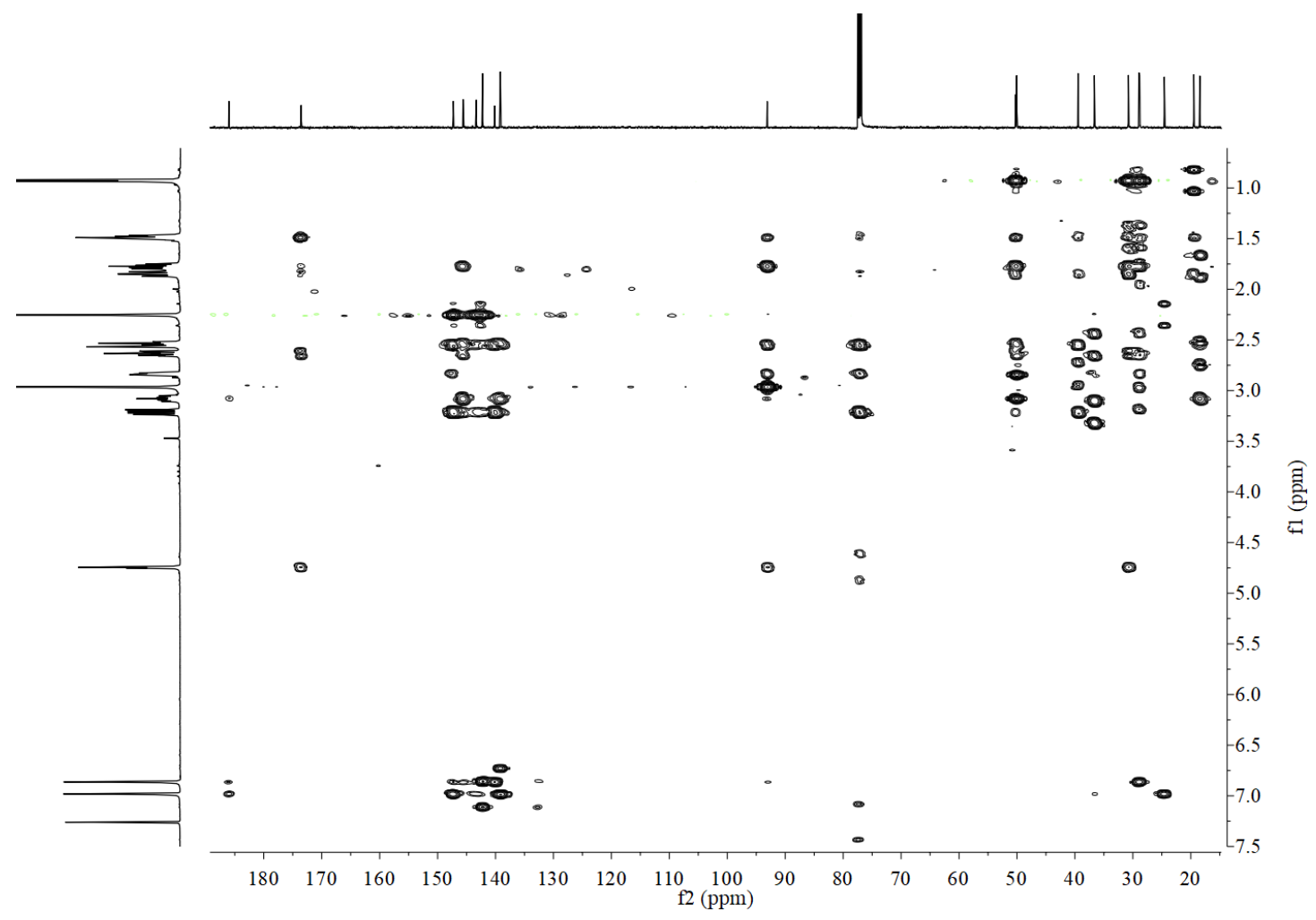


Figure S54. ${ }^{1} \mathrm{H}-{ }^{1} \mathrm{H}$ COSY spectrum of fortalpinoid D (4) in $\mathrm{CDCl}_{3}$.

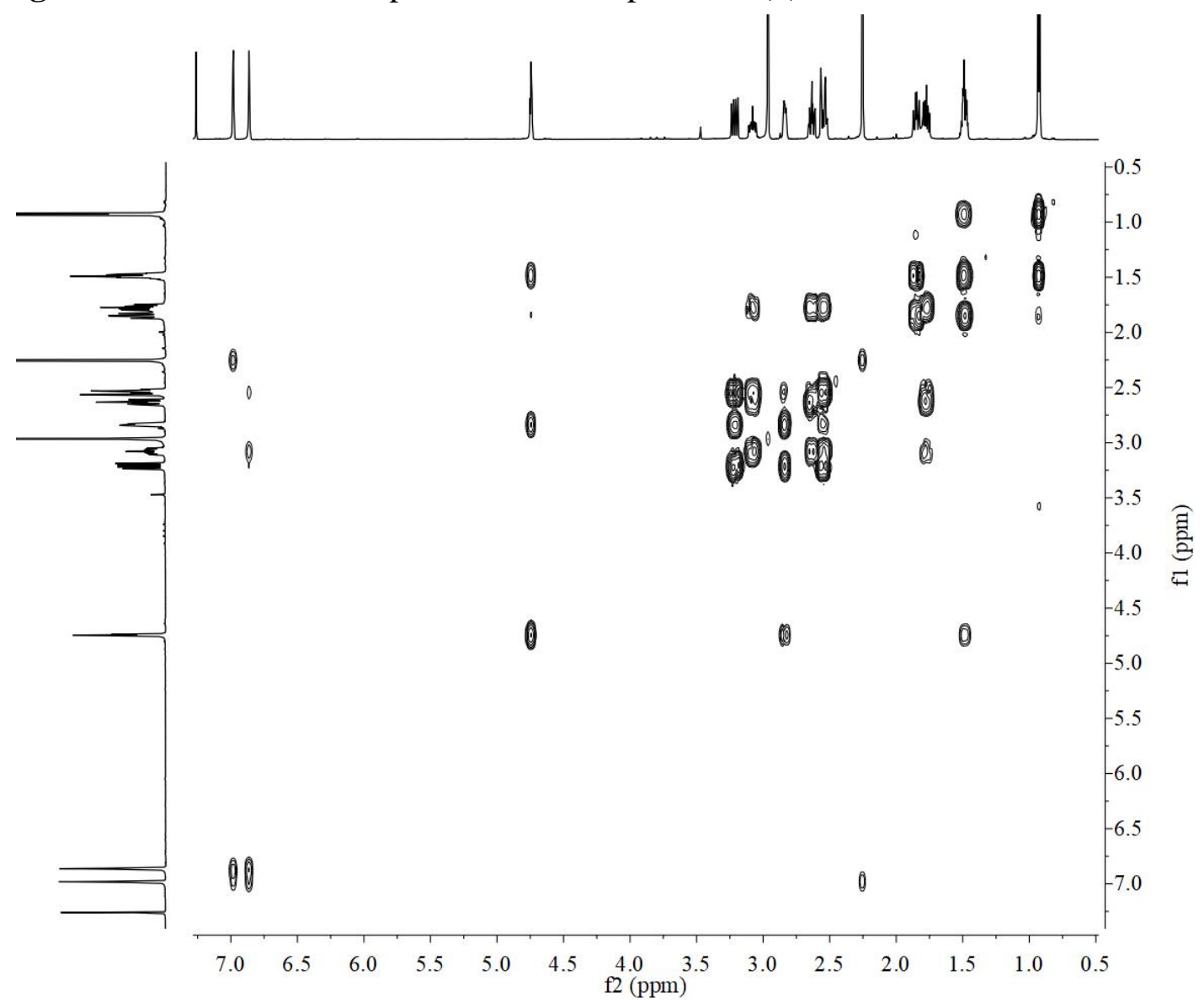

Figure S55. NOESY spectrum of fortalpinoid D (4) in $\mathrm{CDCl}_{3}$.

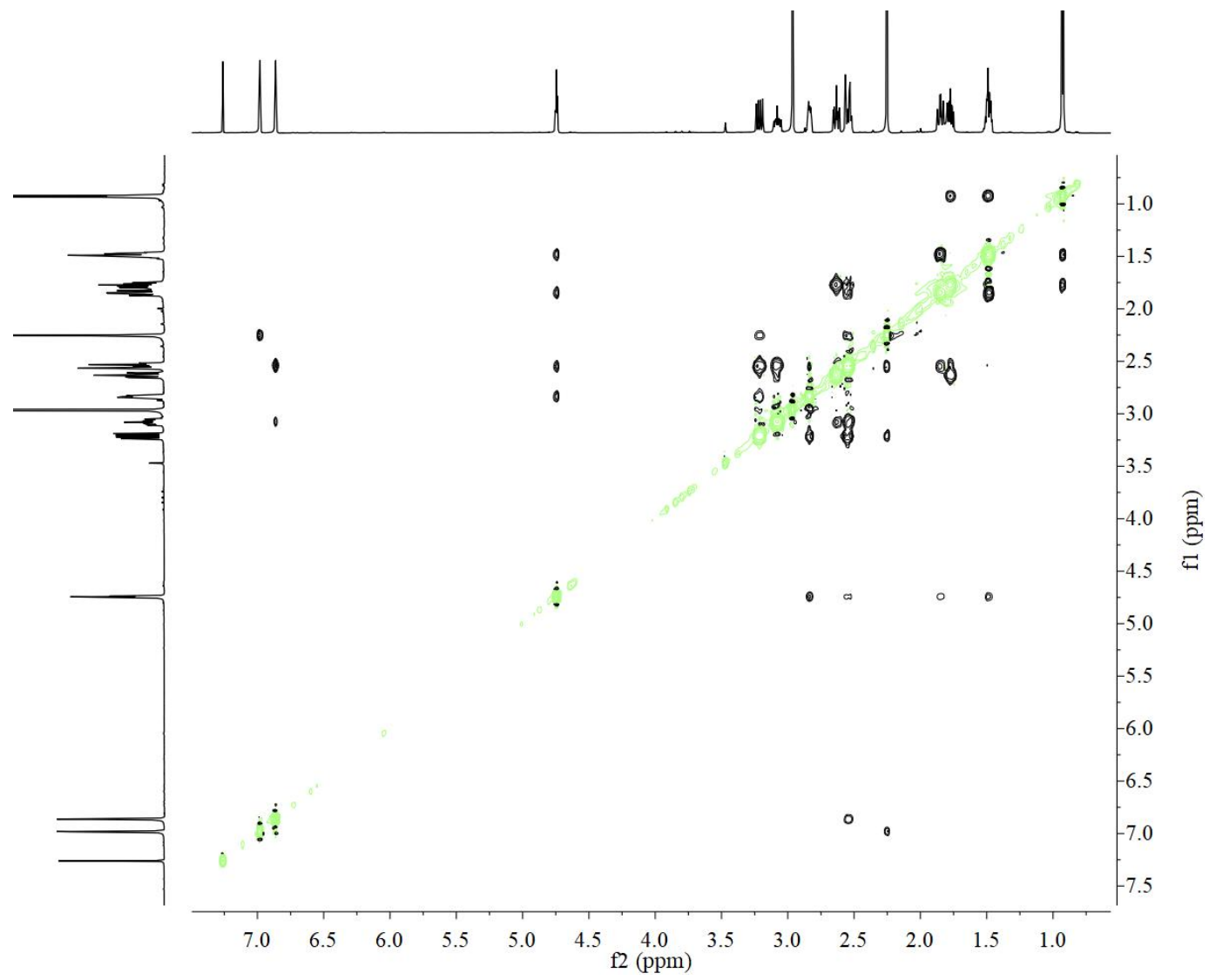


Figure S56. (+)-ESIMS spectrum of fortalpinoid D (4).

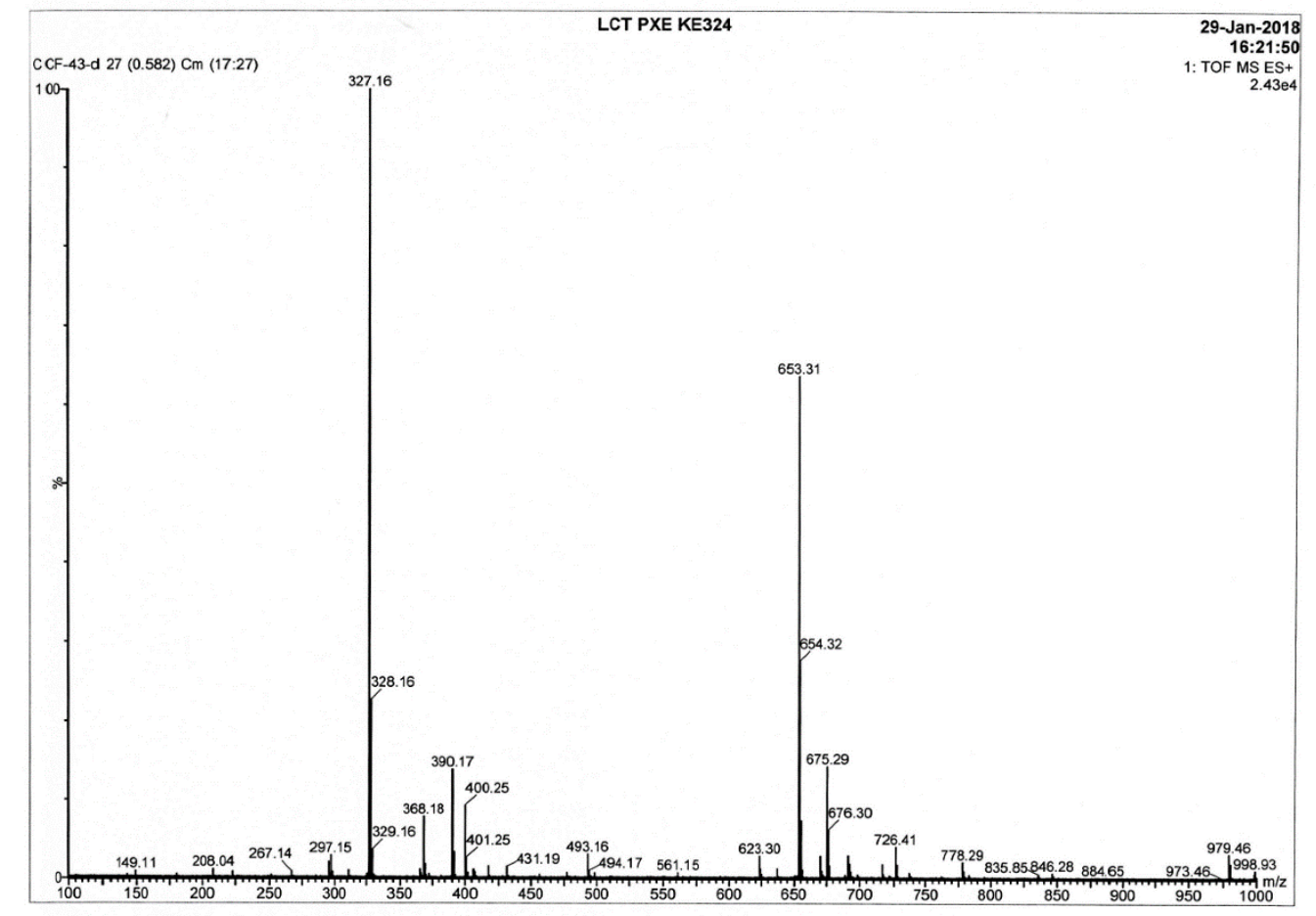

Figure S57. (+)-HRESIMS spectrum of fortalpinoid D (4).

Elemental Composition Report

Page 1

Single Mass Analysis

Tolerance $=3.0$ PPM I DBE: $\min =-1.5, \max =50.0$

Number of isotope peaks used for i-FIT $=3$

Monoisotopic Mass, Even Electron lons

386 formula(e) evaluated with 1 results within limits (up to 50 best isotopic matches for each mass)

$\begin{array}{lll}\text { Elements Used: } & \text { H: } 0-1000 & \text { O: } 0-500 \quad \mathrm{Na}: 0-1\end{array}$

LCT PXE KE 324

29-Jan-2018

1: TOF MS ES+

100 653.3113

CCF-43-d $27(0.582) \mathrm{Cm}(16: 21: 50$

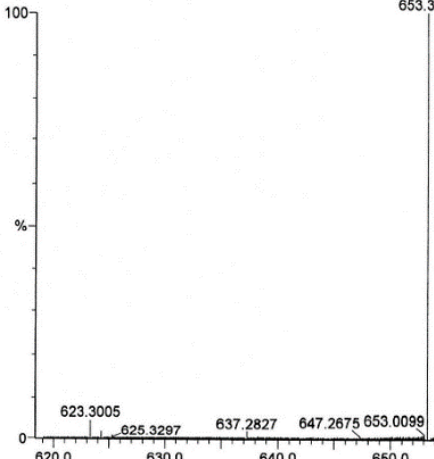

$1.55 \mathrm{e}+004$

Minimum: $\quad 5.0-3.0-1.5$

Mass Calc. Mass mDa PPM DBE i-FIT i-FIT (Norm) Formula

$\begin{array}{llllllllll}653.3113 & 653.3114 & -0.1 & -0.2 & 18.5 & 185.2 & 0.0 & \text { C40 } & \text { H45 } & 08\end{array}$ 
Figure S58. IR spectrum of fortalpinoid.D (4).

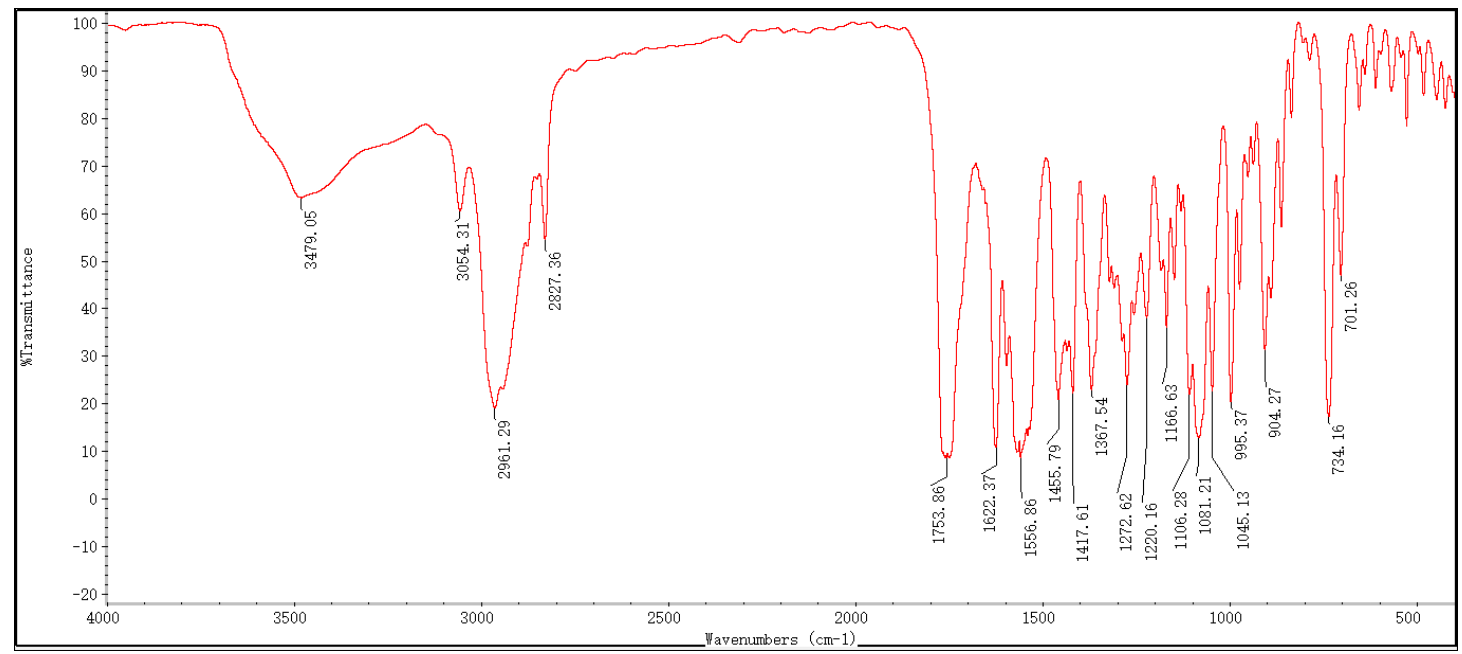

Figure S59. ${ }^{1} \mathrm{H}$ NMR spectrum of fortalpinoid E (5) in pyridine- $d_{5}$.

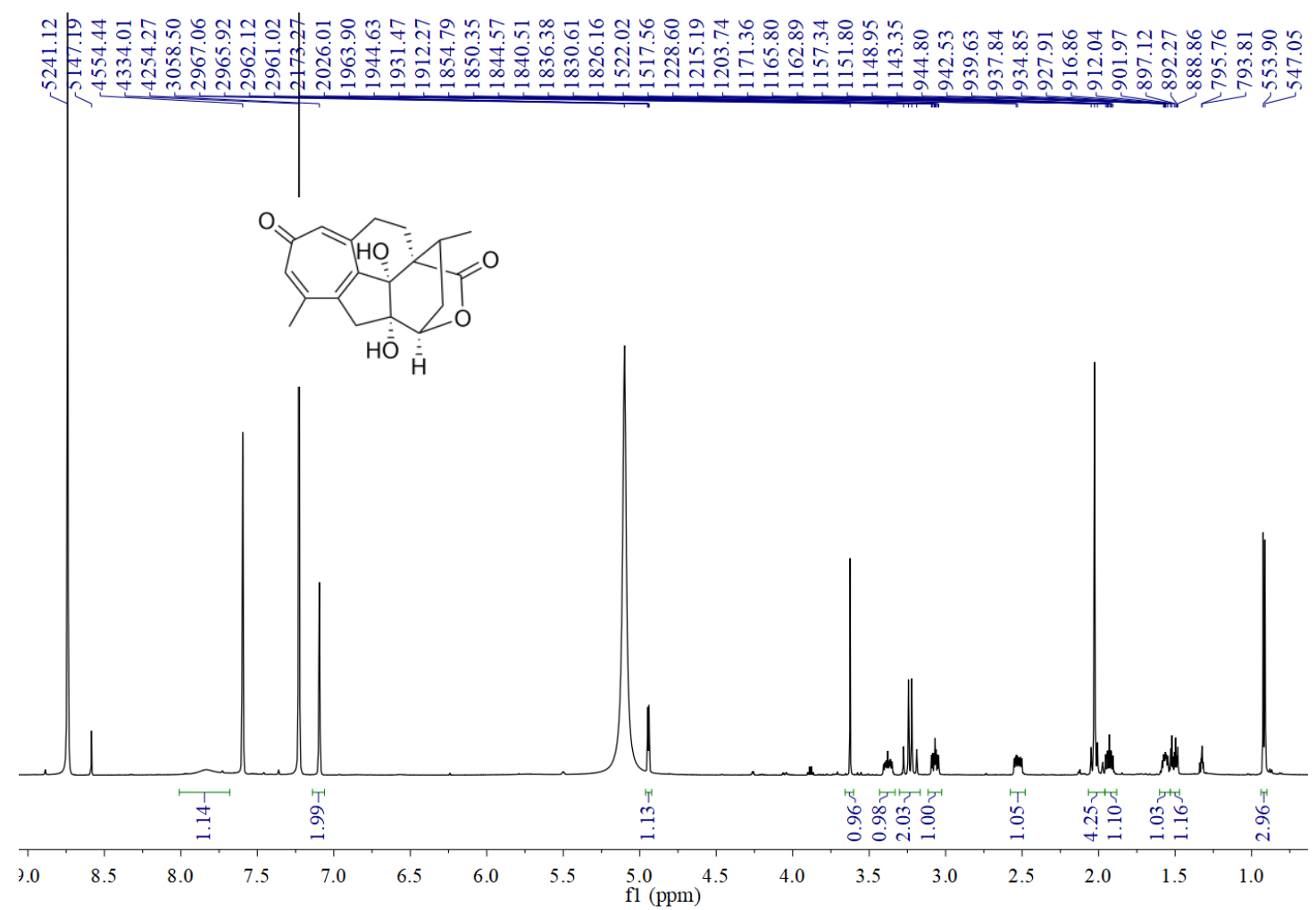


Figure S60. ${ }^{13} \mathrm{C}$ NMR spectrum of fortalpinoid E (5) in pyridine- $d_{5}$.

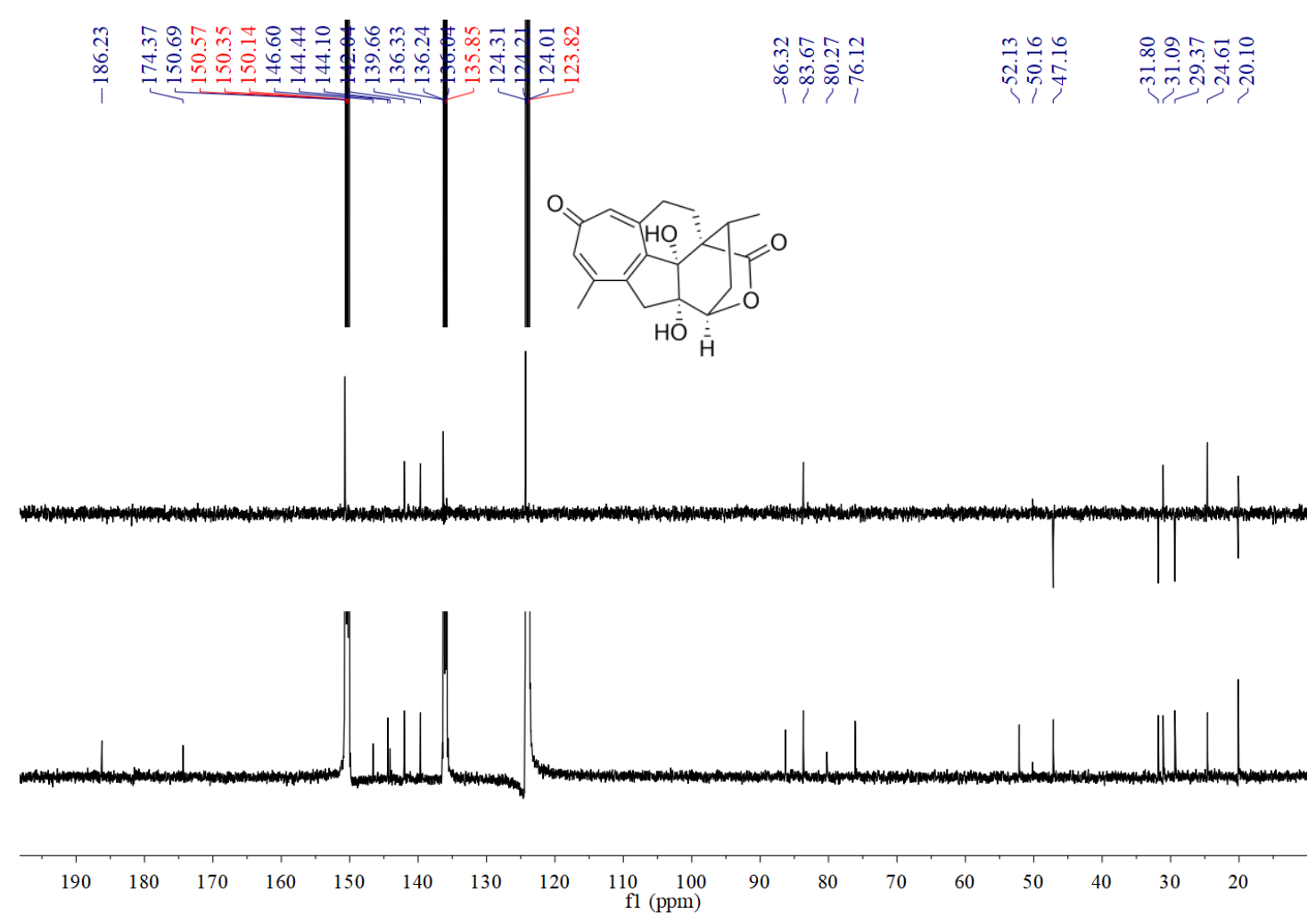

Figure S61. HSQC spectrum of fortalpinoid E (5) in pyridine- $d 5$.

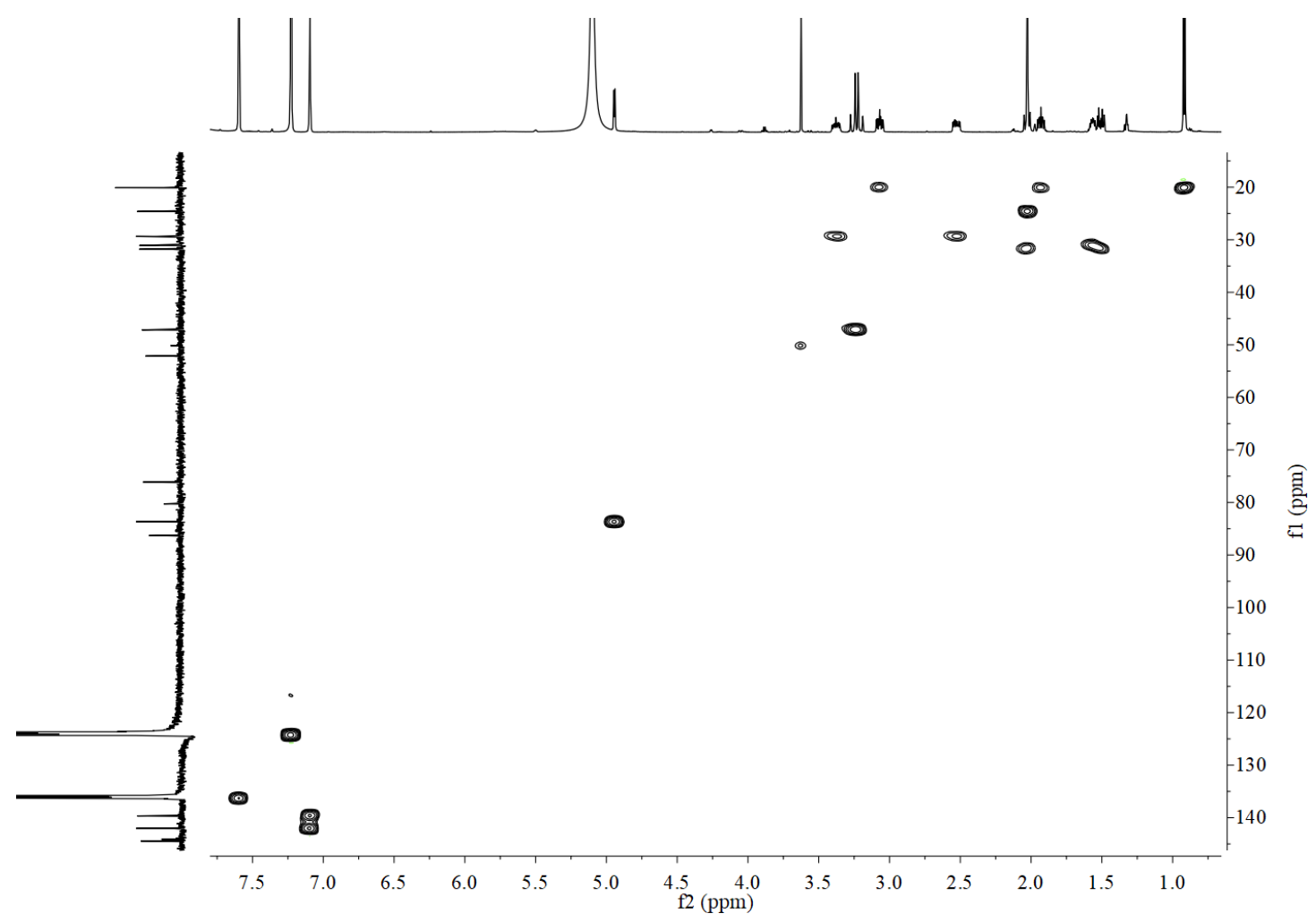


Figure S62. HMBC spectrum of fortalpinoid E (5) in pyridine- $d_{5}$.

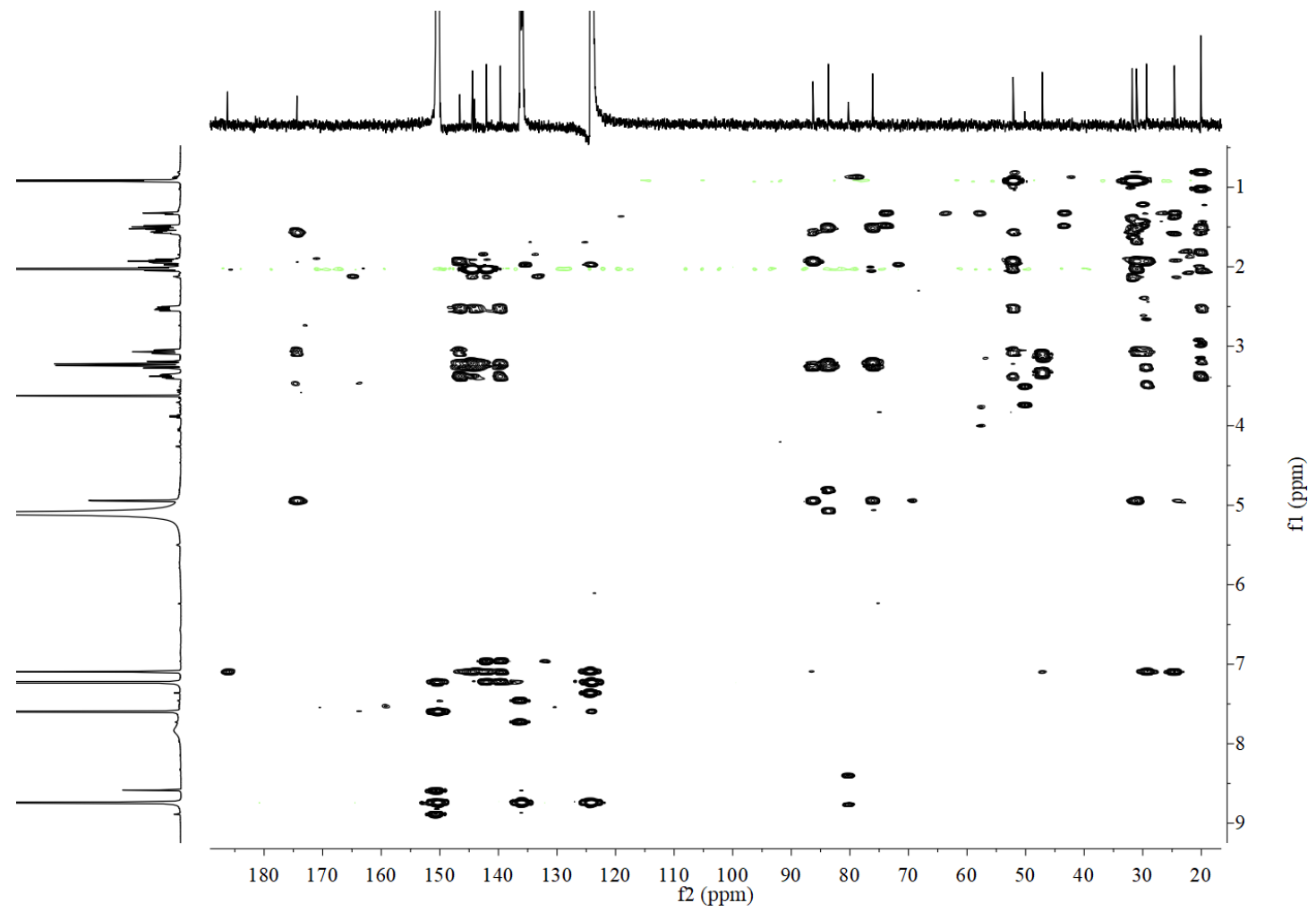

Figure S63. ${ }^{1} \mathrm{H}-{ }^{1} \mathrm{H}$ COSY spectrum of fortalpinoid E (5) in pyridine- $d_{5}$.

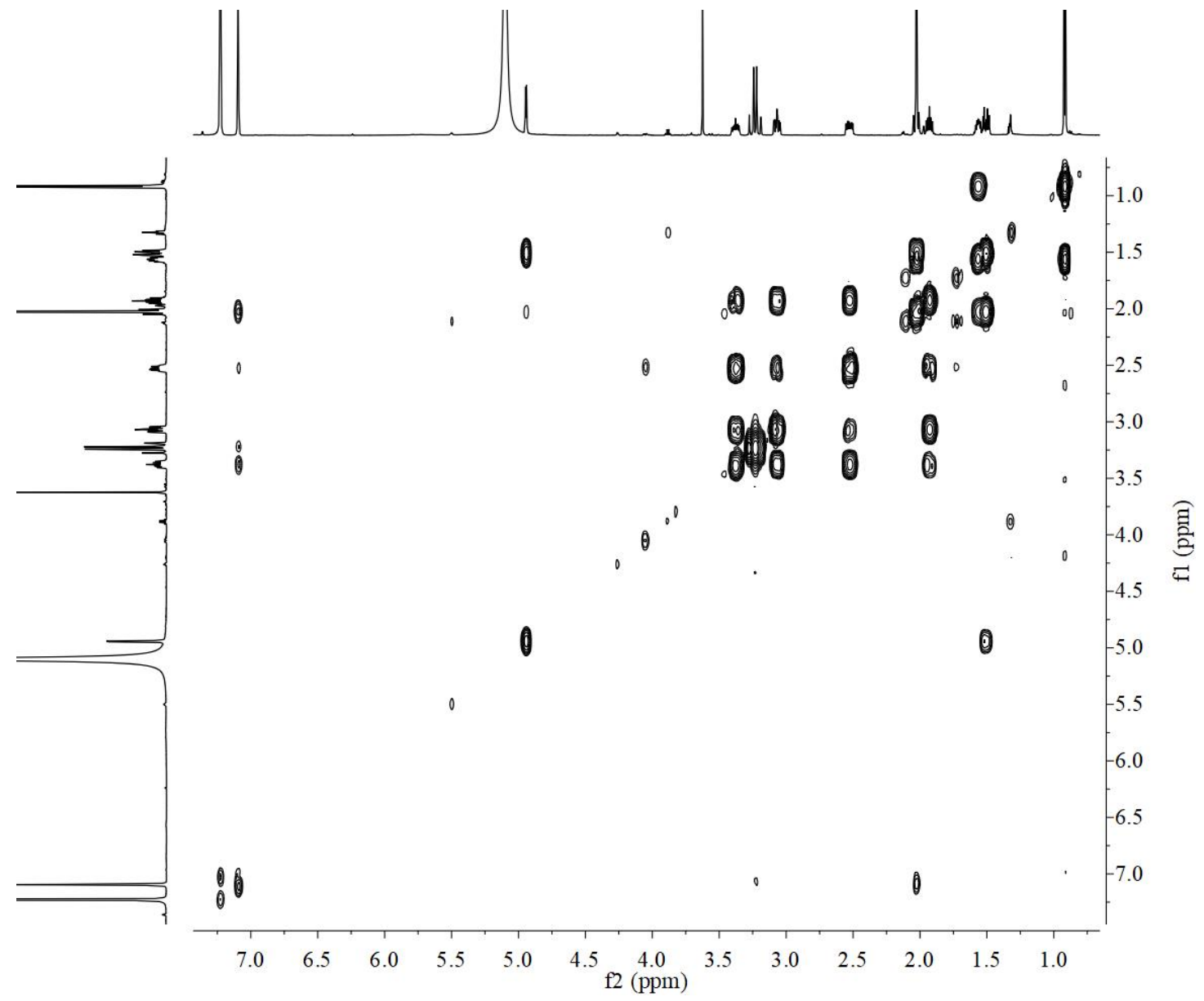


Figure S64. NOESY spectrum of fortalpinoid E (5) in pyridine- $d_{5}$.

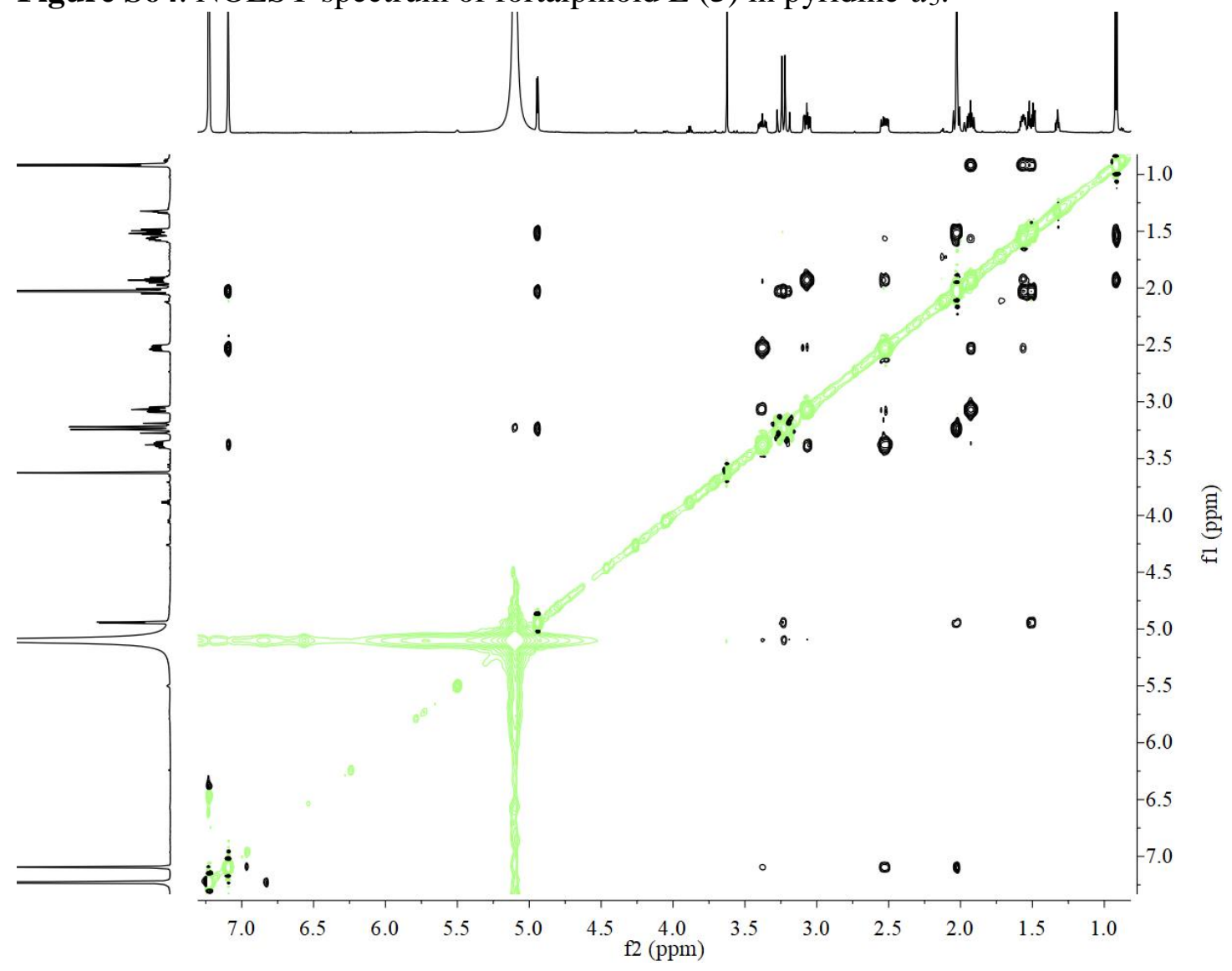

Figure S65. (+)-ESIMS spectrum of fortalpinoid E (5).

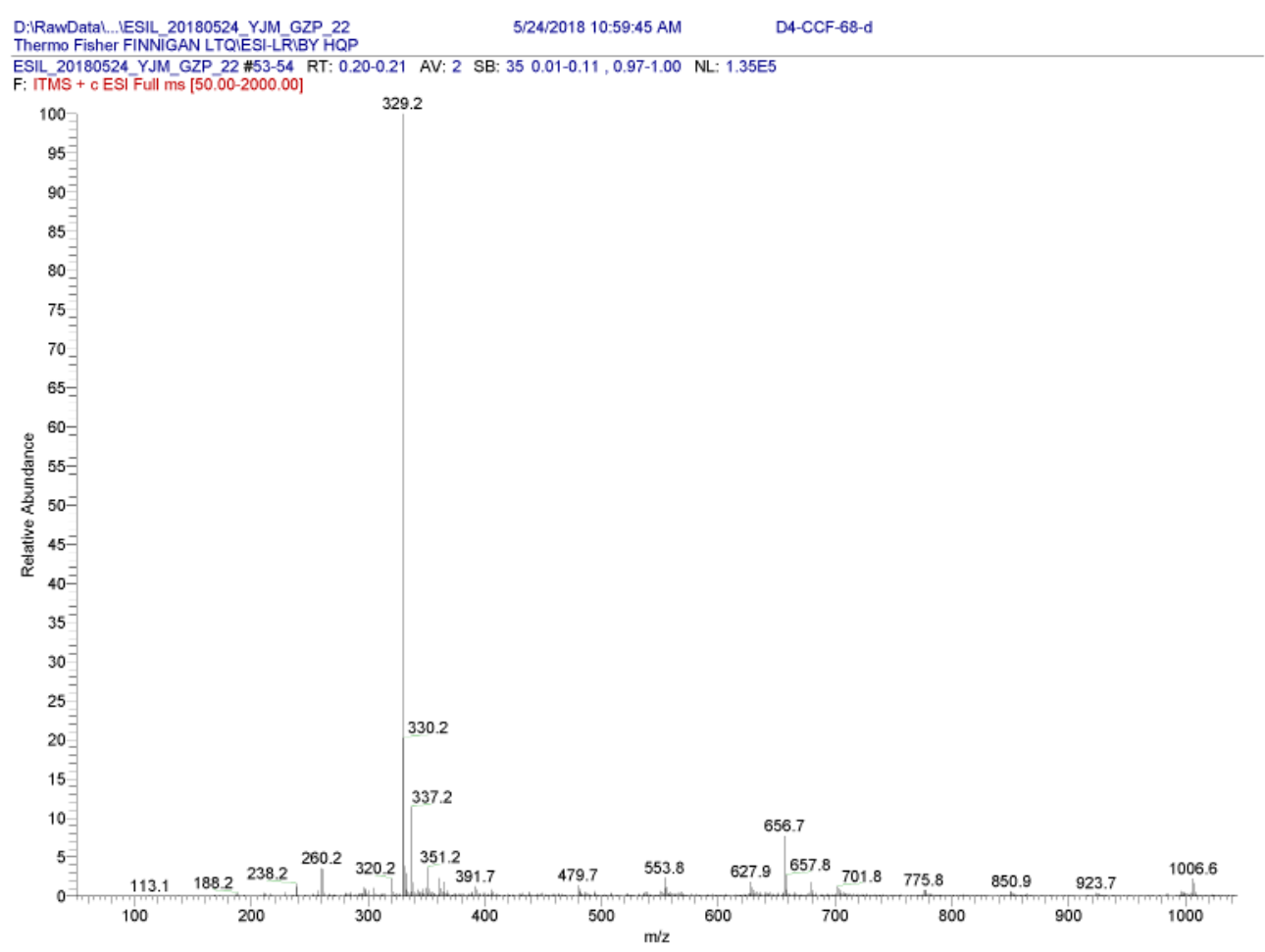


Figure S66. (-)-ESIMS spectrum of fortalpinoid E (5).

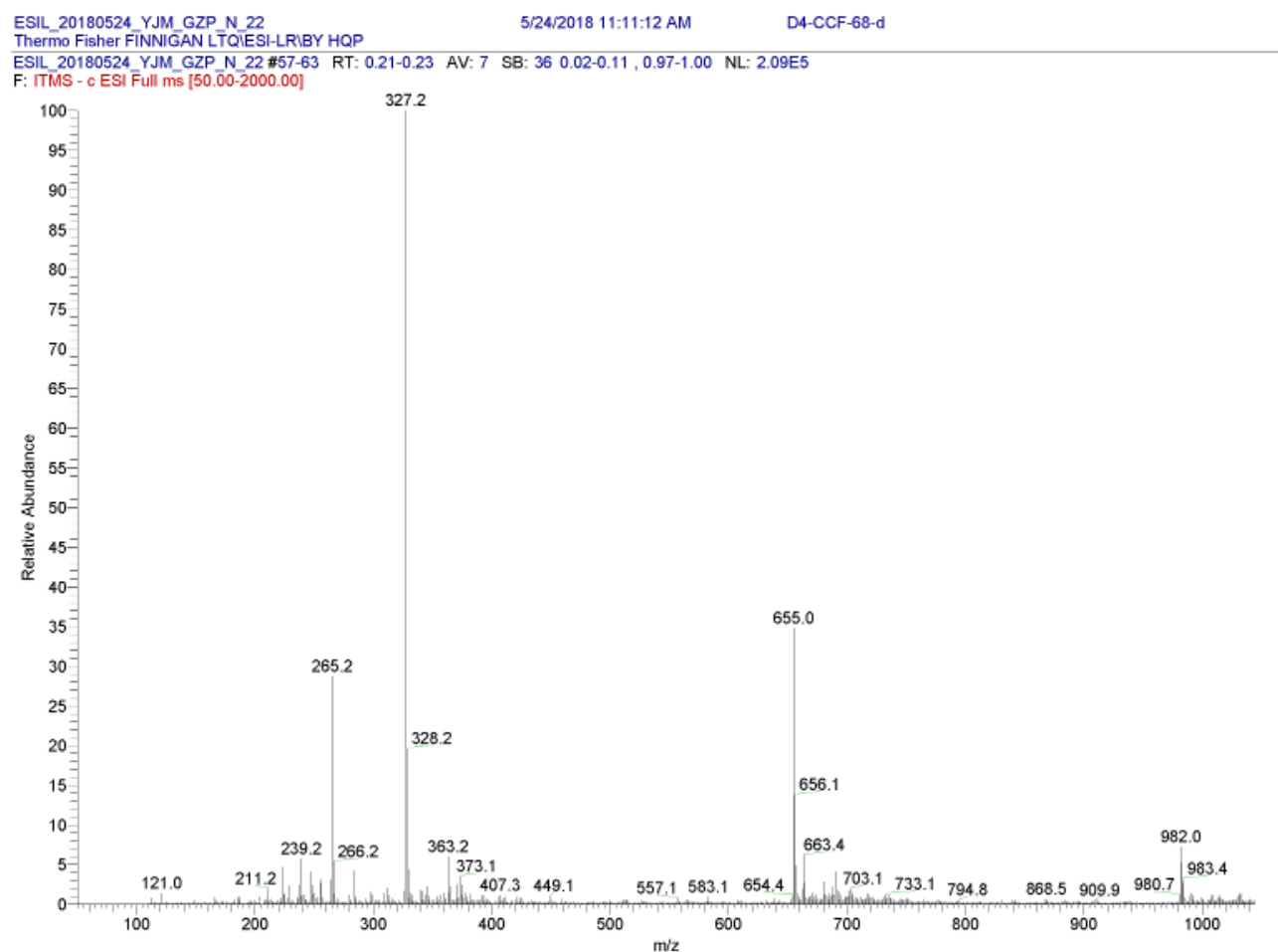

Figure S67. (+)-HRESIMS spectrum of fortalpinoid E (5).

Qualitative Analysis Report

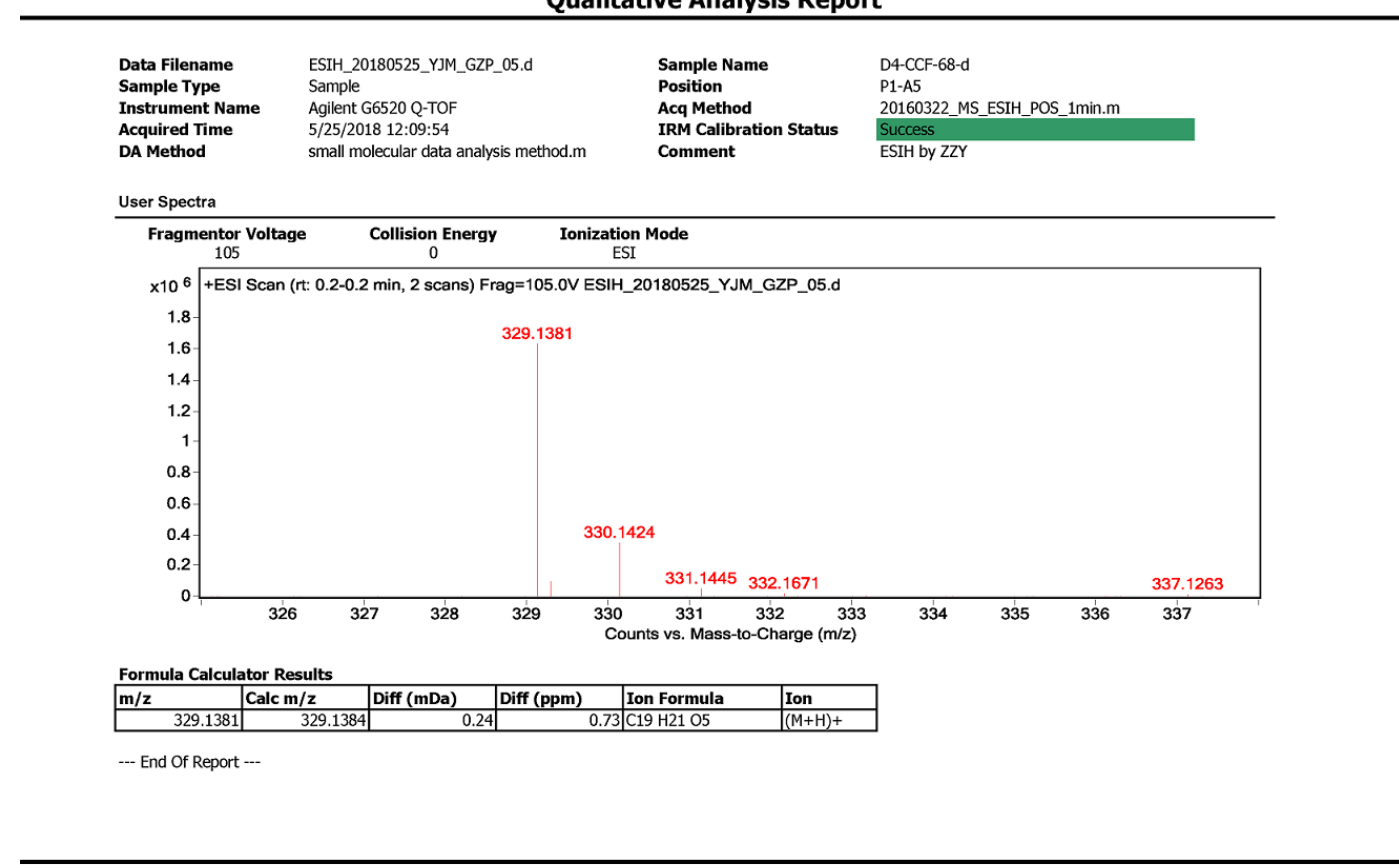

A. Agilent Technologies

Page 1 of 1

Printed at: 12:13 on: 5/25/2018 
Figure S68. IR spectrum of fortalpinoid E (5).

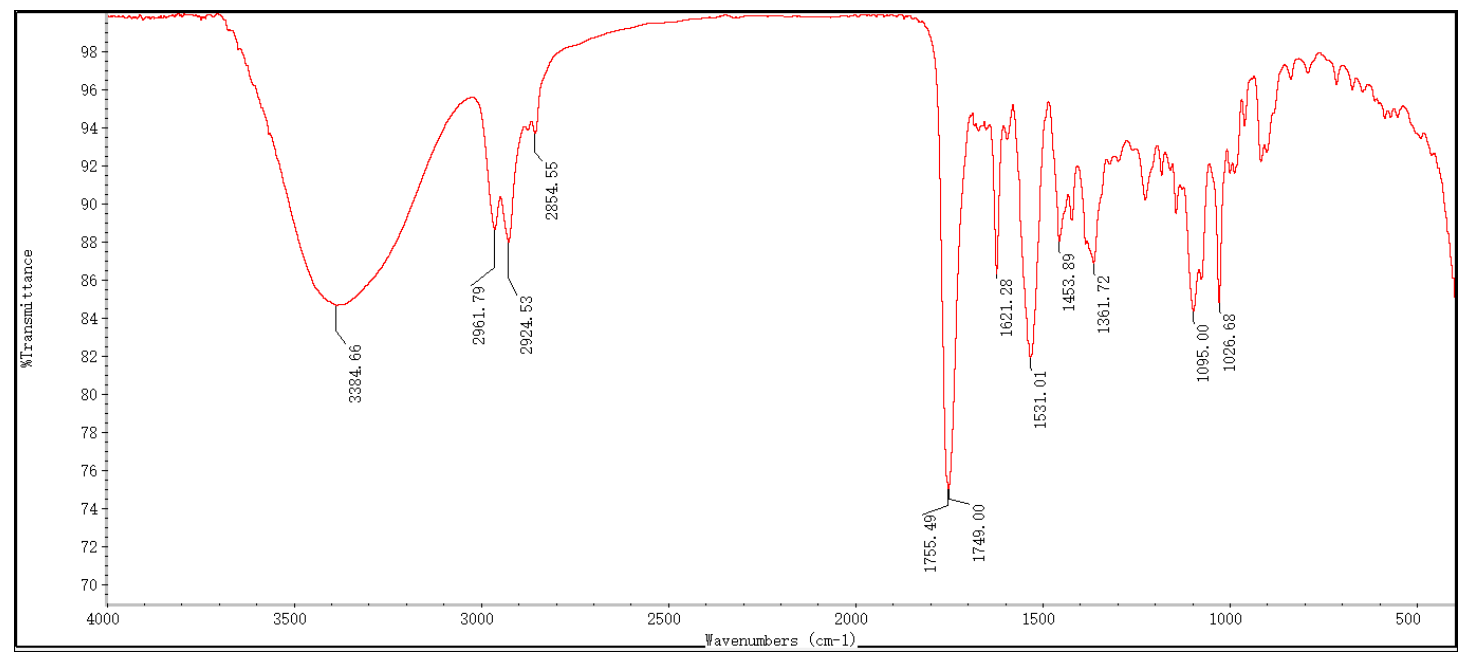

Figure S69. ${ }^{1} \mathrm{H}$ NMR spectrum of fortalpinoid $\mathrm{F}(6)$ in $\mathrm{CDCl}_{3}$.

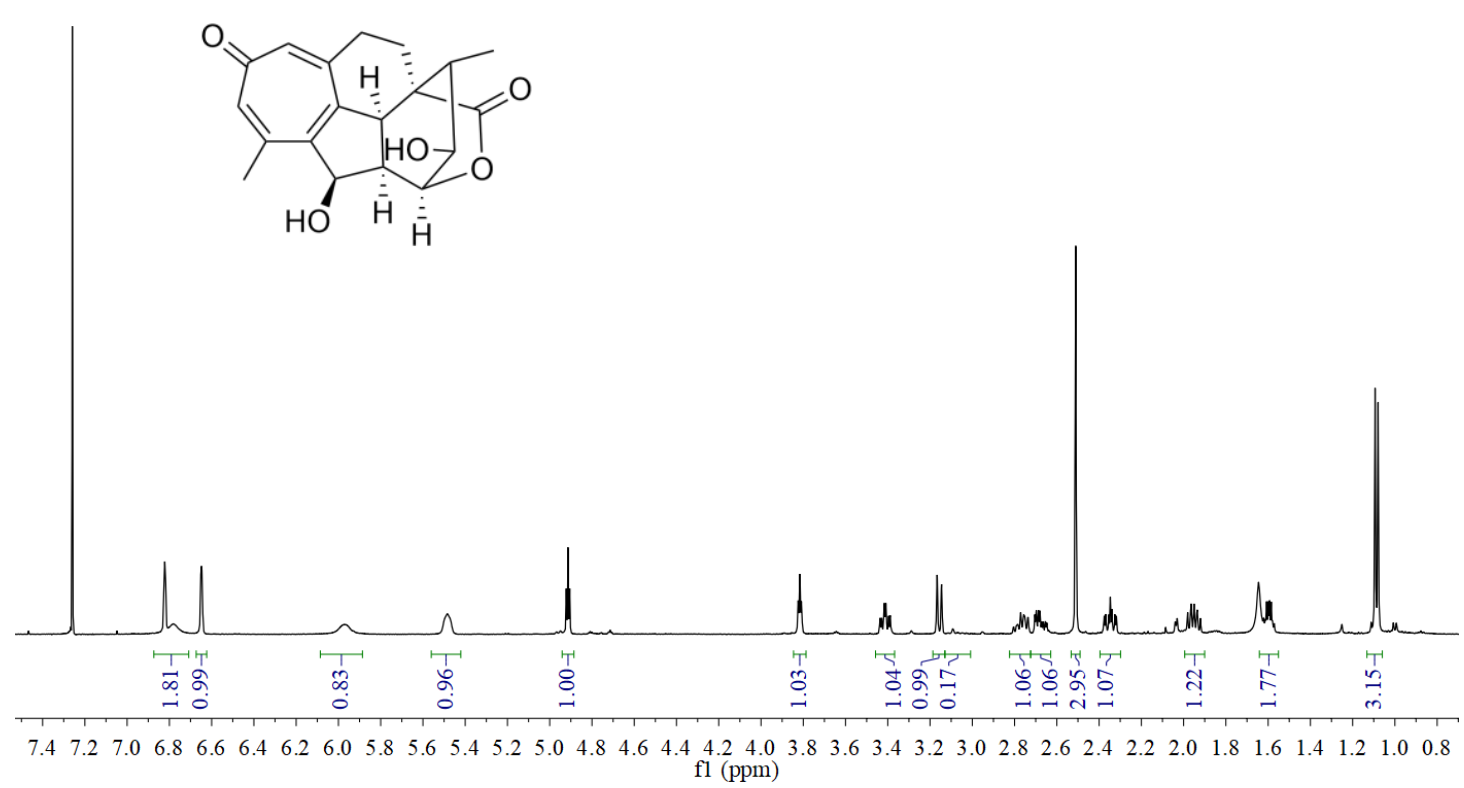


Figure S70. ${ }^{1} \mathrm{H}$ NMR spectrum of fortalpinoid F (6) in pyridine- $d 5$.

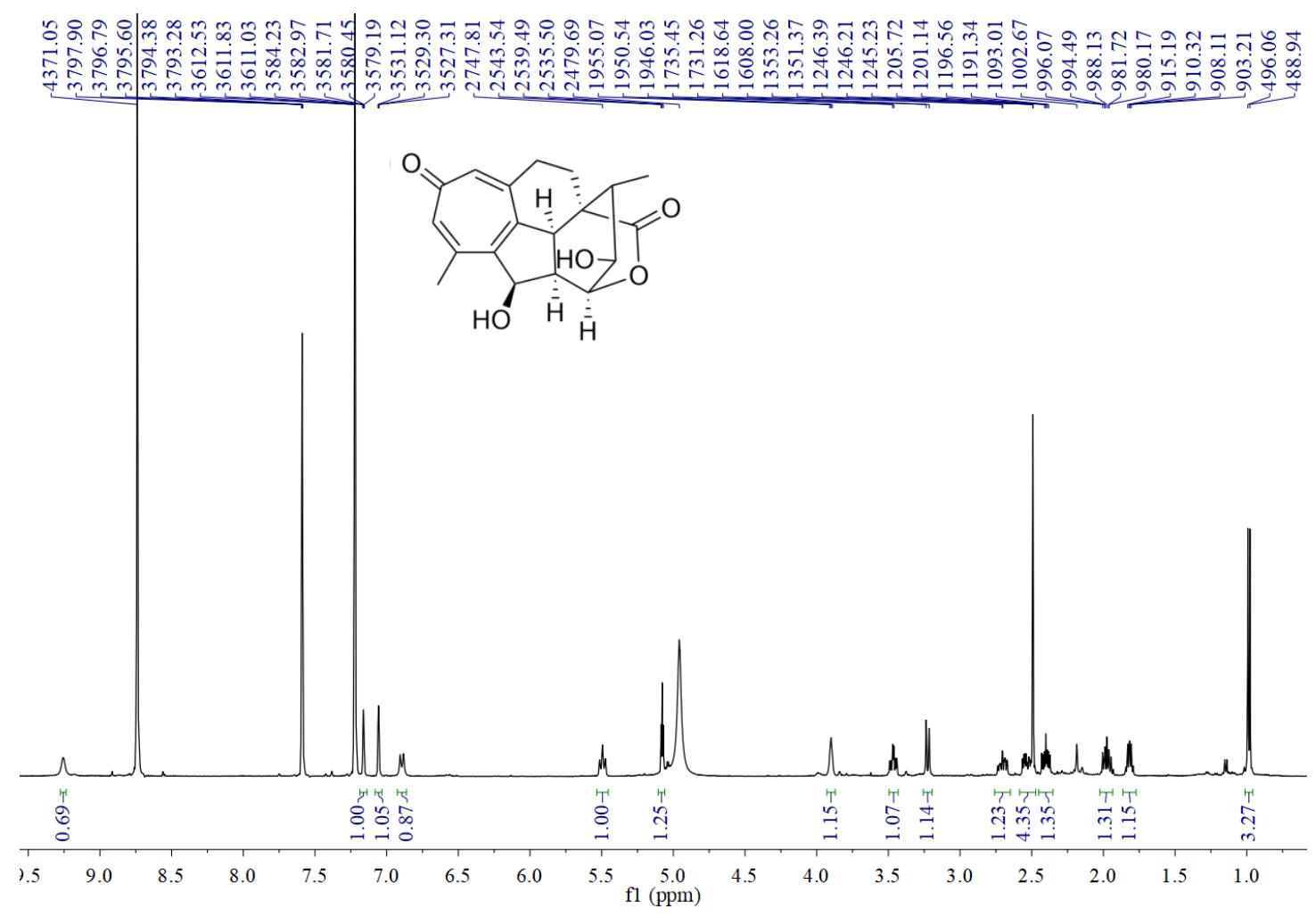

Figure S71. ${ }^{13} \mathrm{C}$ NMR spectrum of fortalpinoid $\mathrm{F}(\mathbf{6})$ in $\mathrm{CDCl}_{3}$.
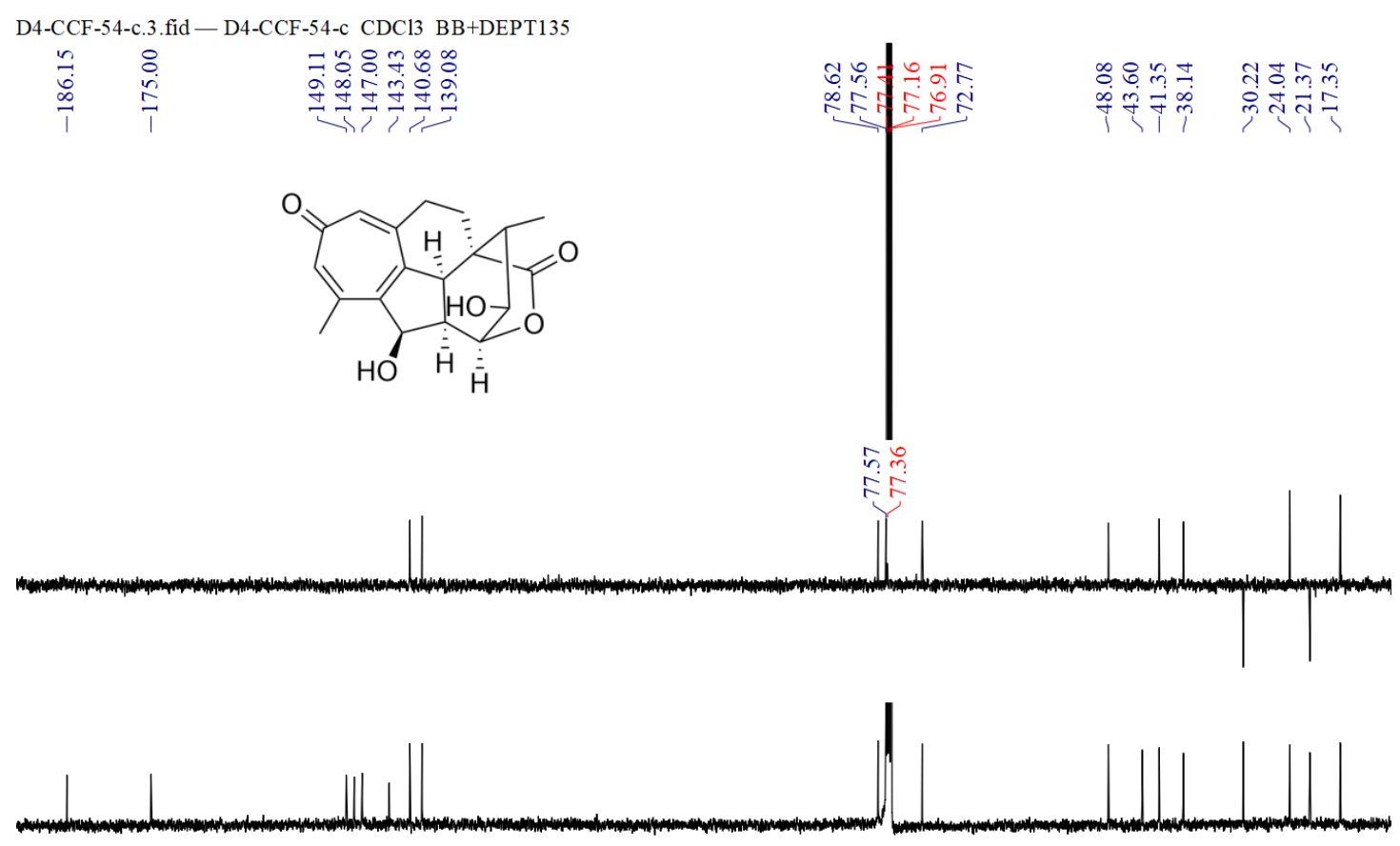

$\begin{array}{llllllllll}190 & 180 & 170 & 160 & 150 & 140 & 130 & 120 & 110 & \begin{array}{l}100 \\ \mathrm{fl}(\mathrm{ppm})\end{array}\end{array}$ 
Figure S72. HSQC spectrum of fortalpinoid $\mathrm{F}(6)$ in $\mathrm{CDCl}_{3}$.

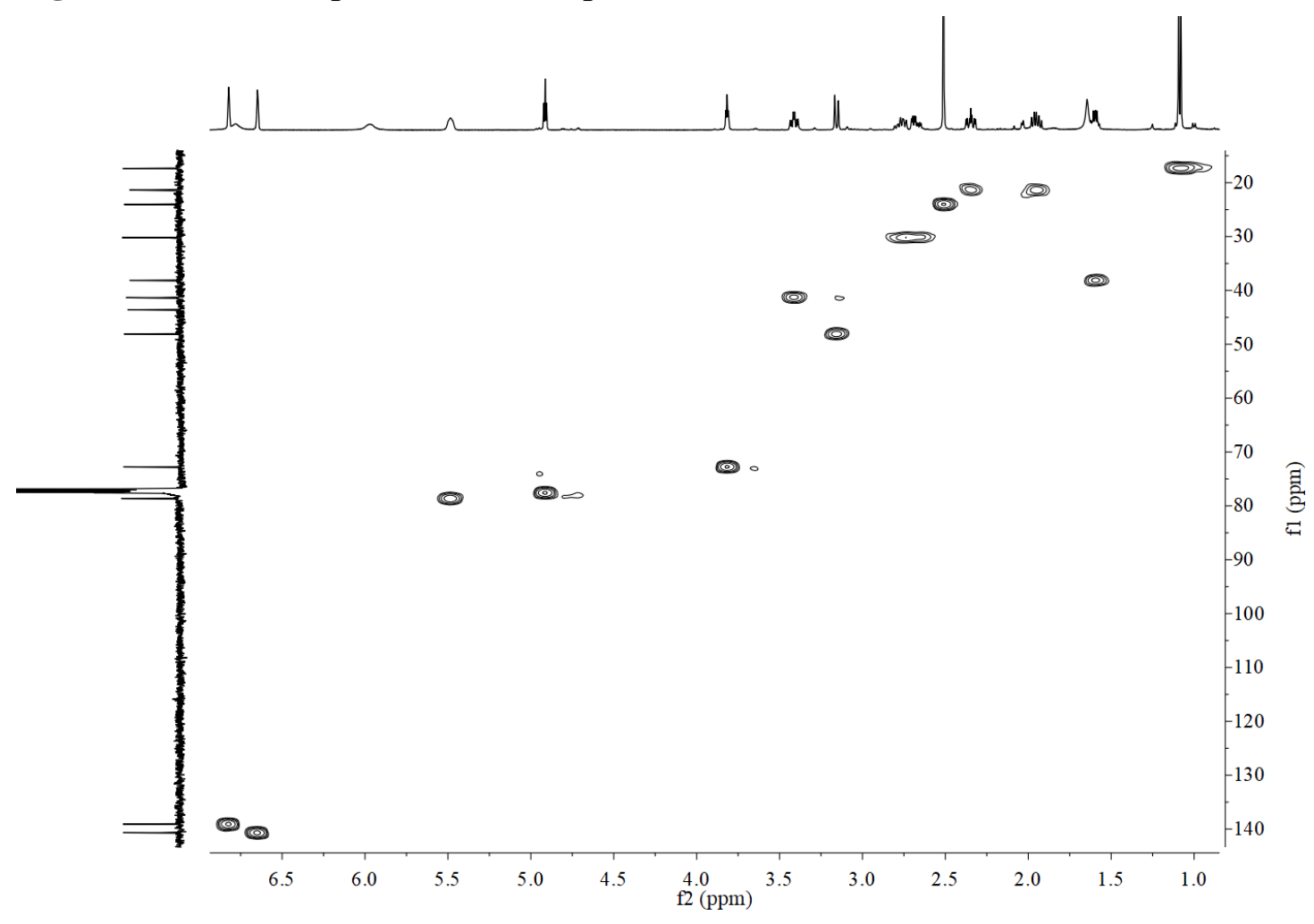

Figure S73. HMBC spectrum of fortalpinoid $\mathrm{F}(\mathbf{6})$ in $\mathrm{CDCl}_{3}$.

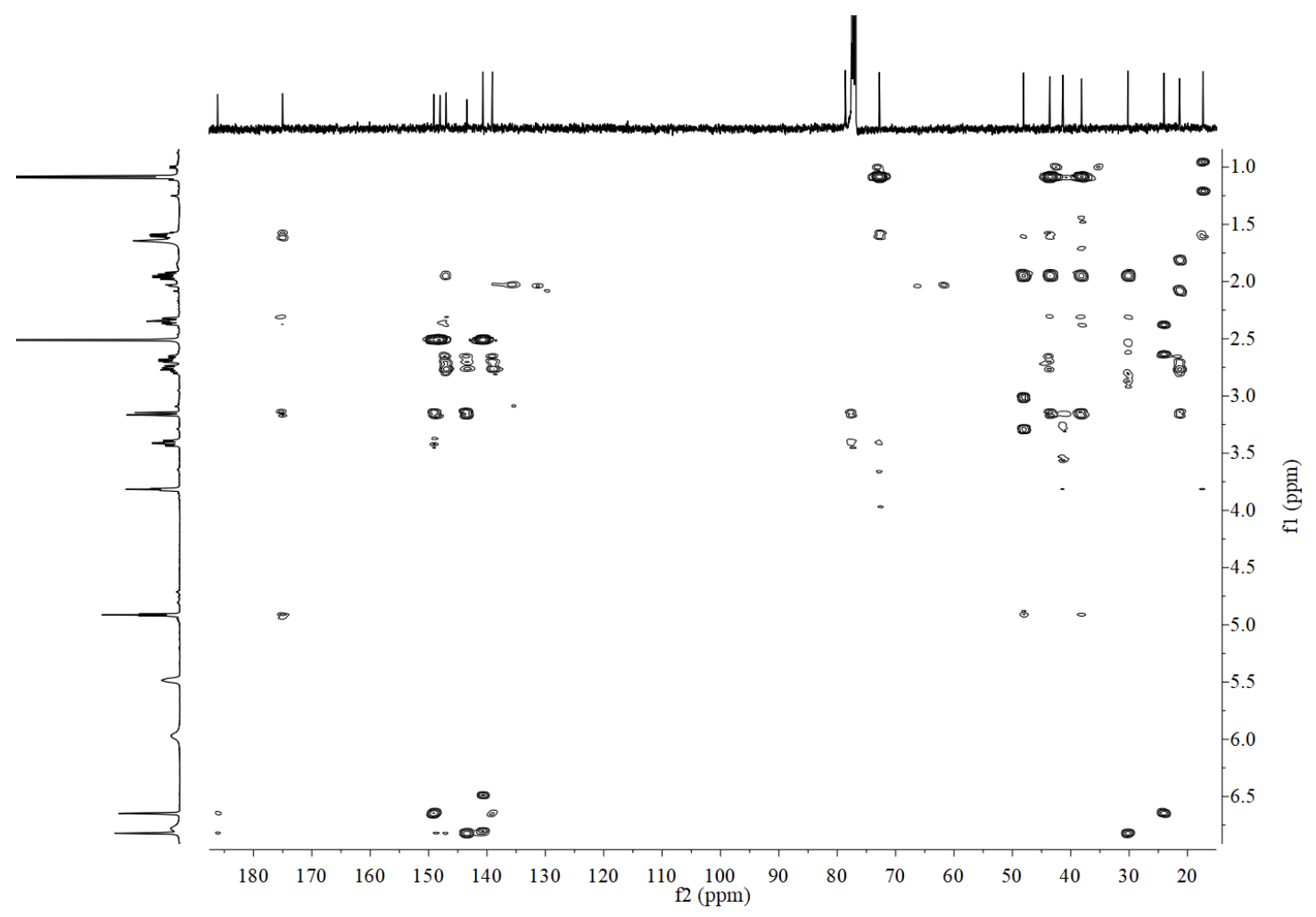


Figure S74. ${ }^{1} \mathrm{H}-{ }^{1} \mathrm{H}$ COSY spectrum of fortalpinoid $\mathrm{F}(6)$ in $\mathrm{CDCl}_{3}$.

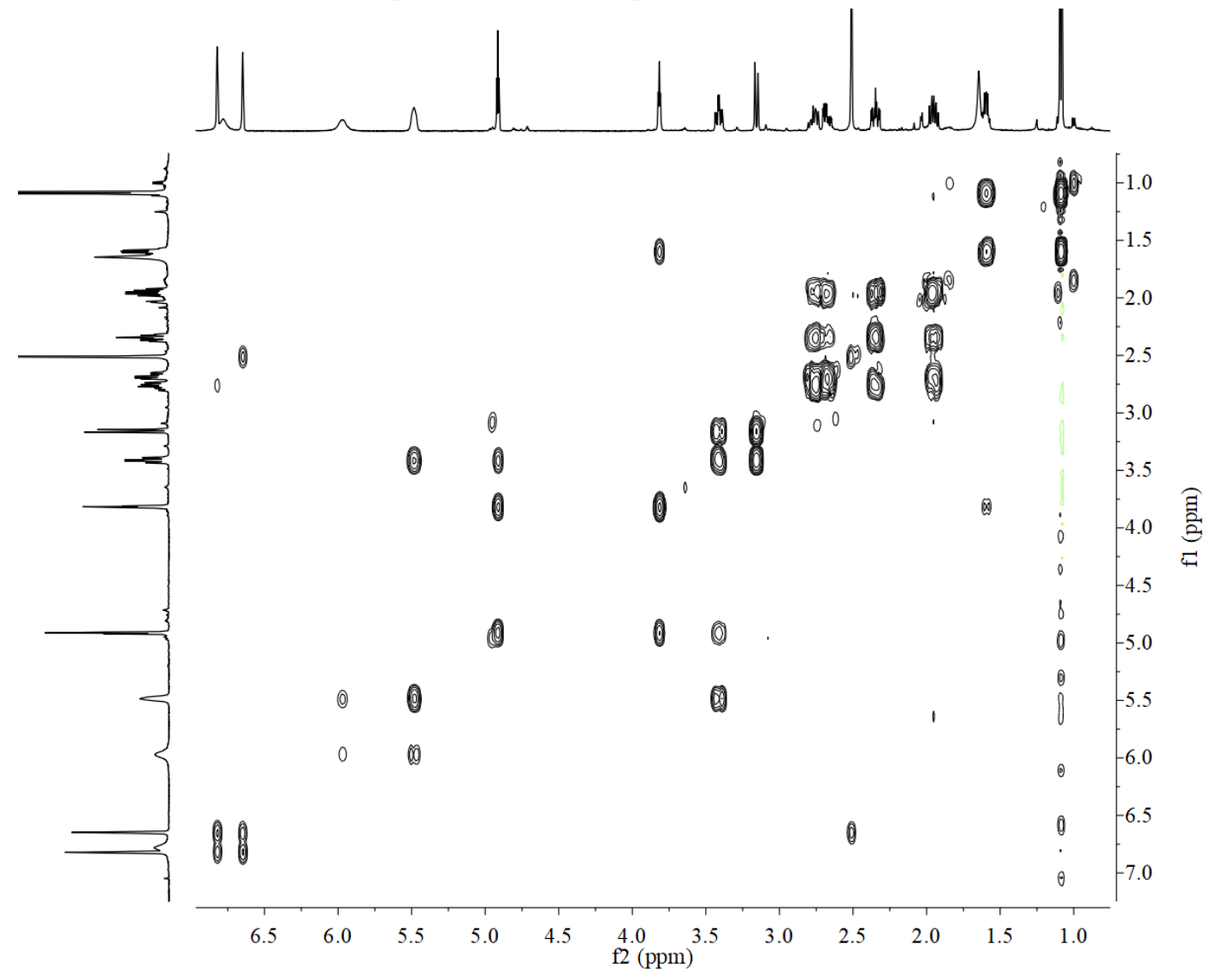

Figure S75. NOESY spectrum of fortalpinoid $\mathrm{F}(\mathbf{6})$ in $\mathrm{CDCl}_{3}$.

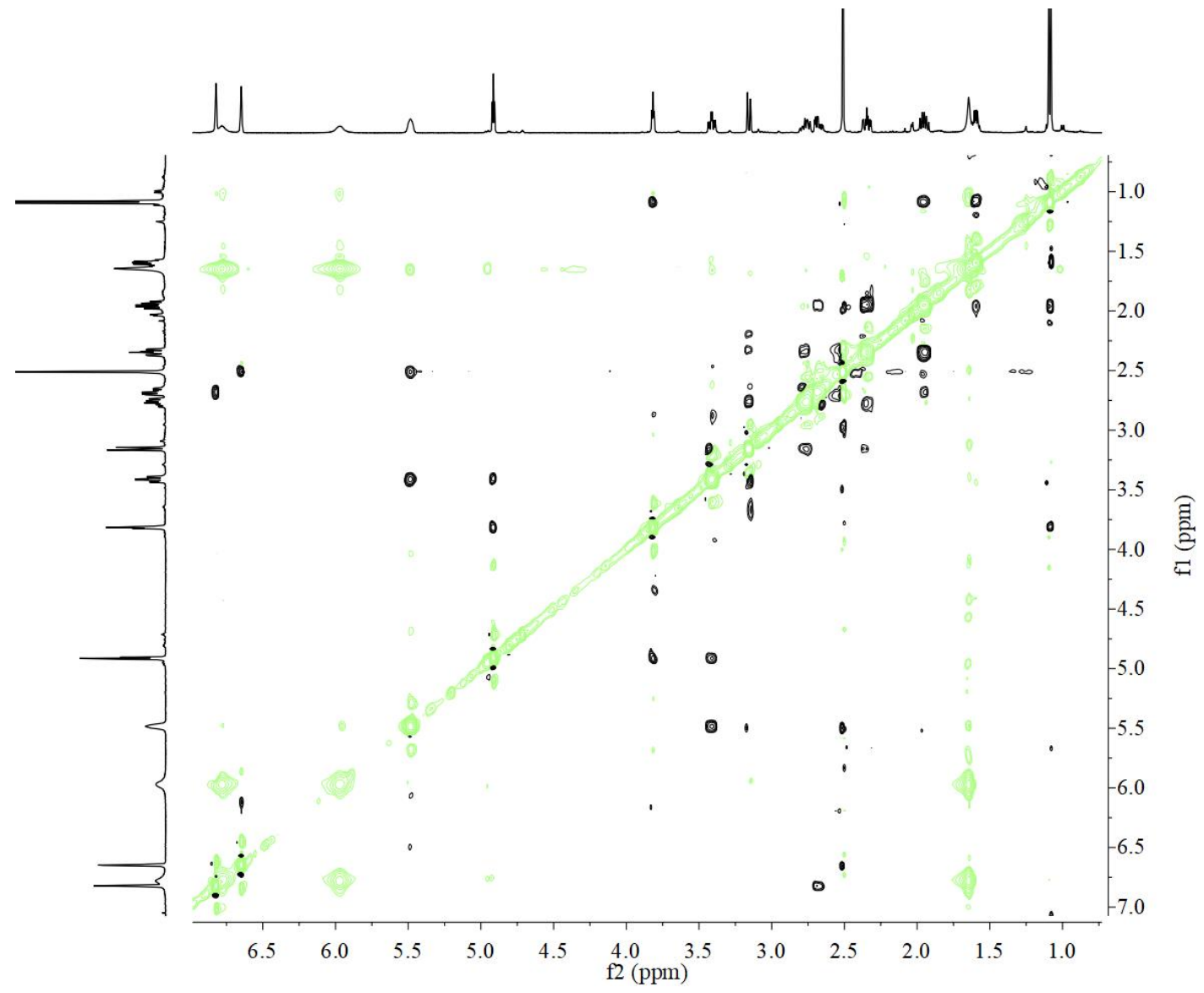


Figure S76. NOESY spectrum of fortalpinoid F (6) in pyridine- $d 5$.

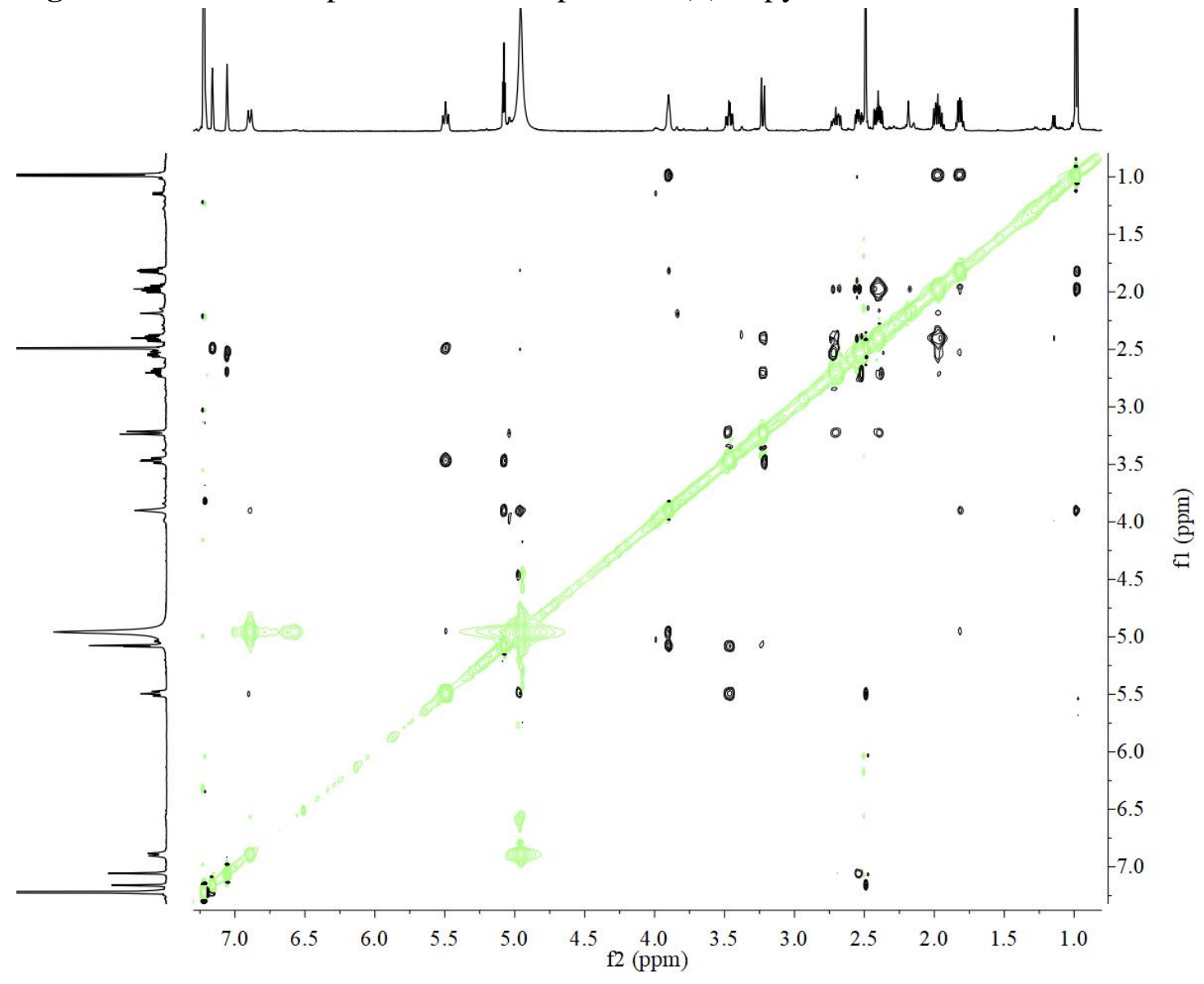

Figure S77. (+)-ESIMS spectrum of fortalpinoid F (6).

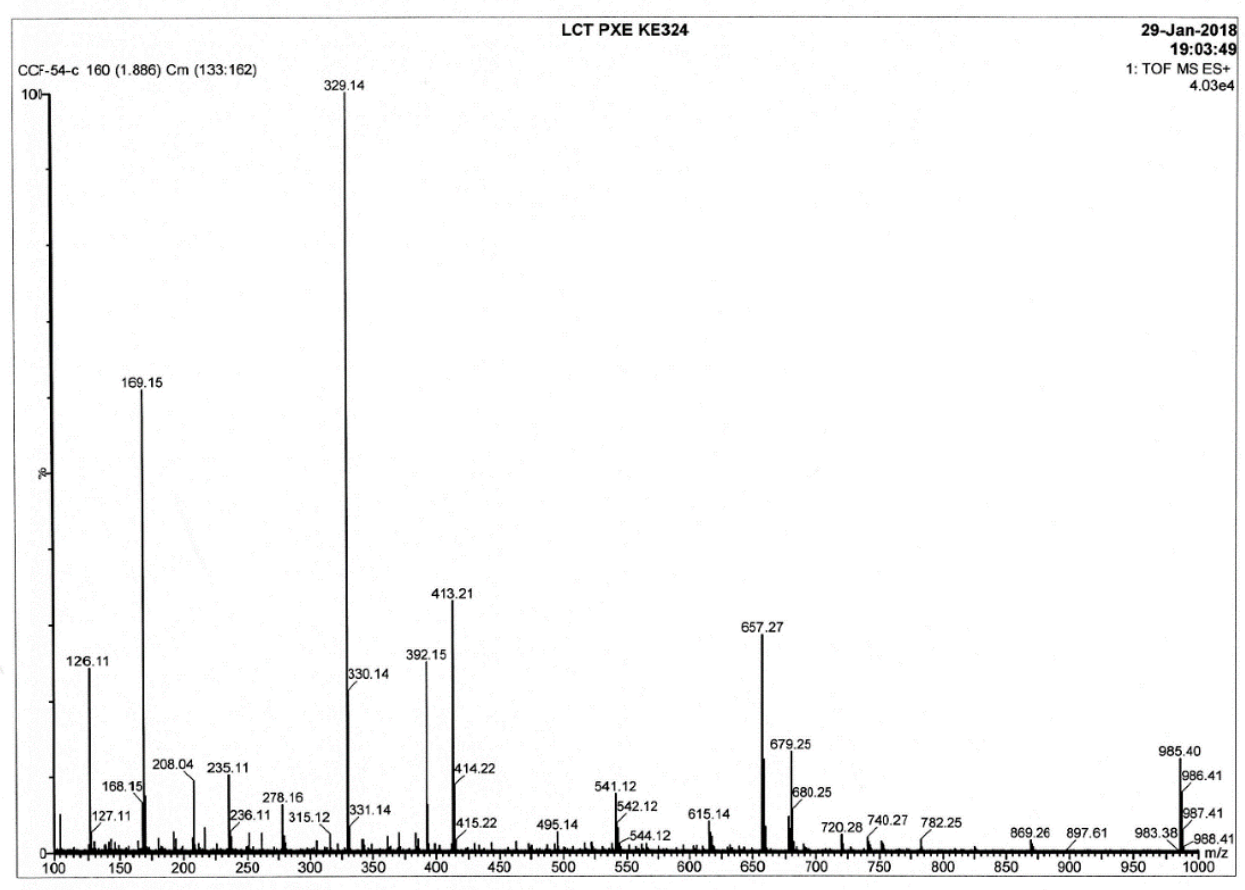


Figure S78. (-)-ESIMS spectrum of fortalpinoid F (6).

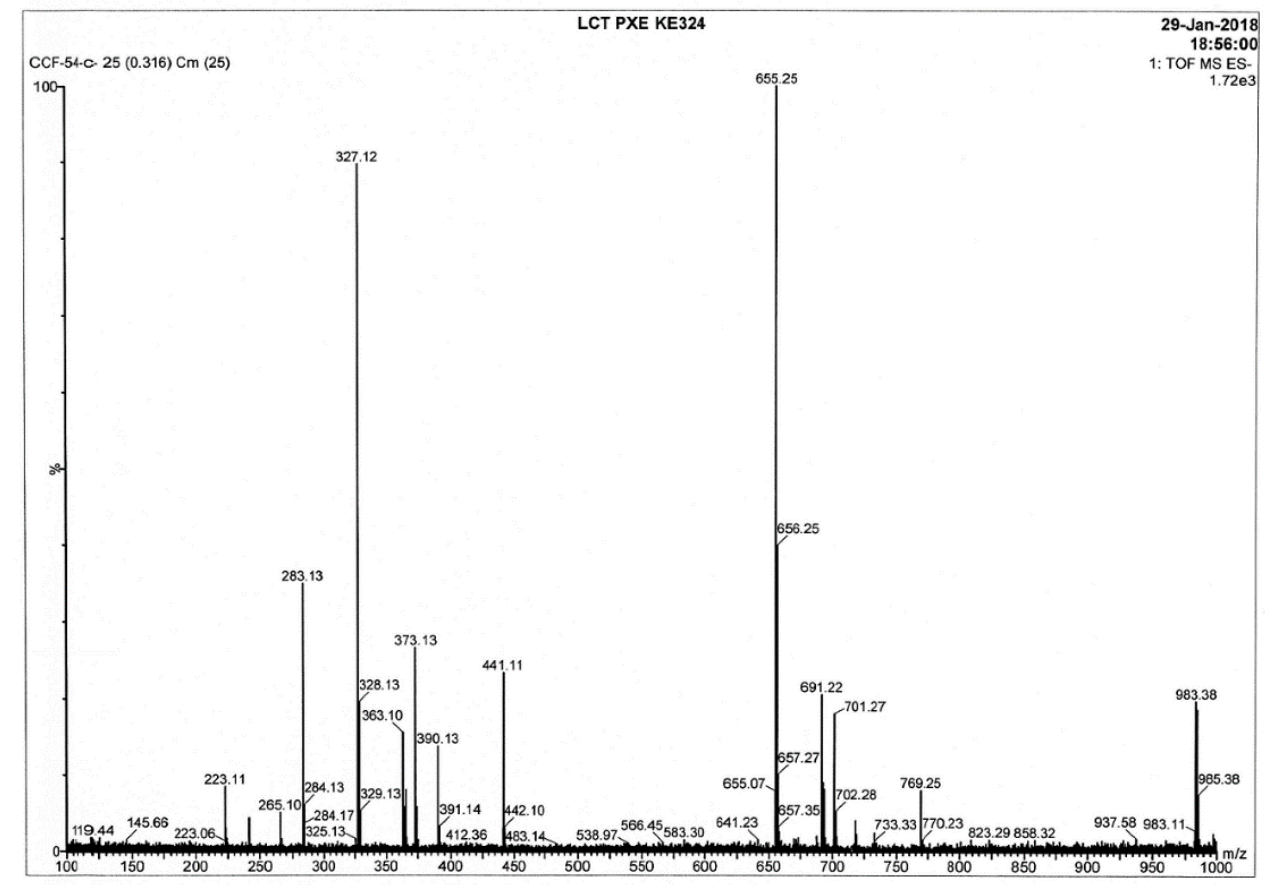

Figure S79. (+)-HRESIMS spectrum of fortalpinoid F (6).

\section{Single Mass Analysis}

Tolerance $=3.0$ PPM I DBE: $\min =-1.5, \max =50.0$

Element prediction: Off

Number of isotope peaks used for $\mathrm{i}-\mathrm{FIT}=3$

Monoisotopic Mass, Even Electron lons

115 formula(e) evaluated with 1 results within limits (up to 50 best isotopic matches for each mass)

Elements Used:

$\begin{array}{lll}\text { C: } 0-100 & \text { H: } 0-1000 & 0: 0-500 \\ \mathrm{Na}\end{array}$

LCT PXE KE 324

29-Jan-2018

CCF-54-c $160(1.886)$ Cm $\begin{array}{r}19: 03: 49 \\ (132: 160)\end{array}$

1: TOF MS ES+

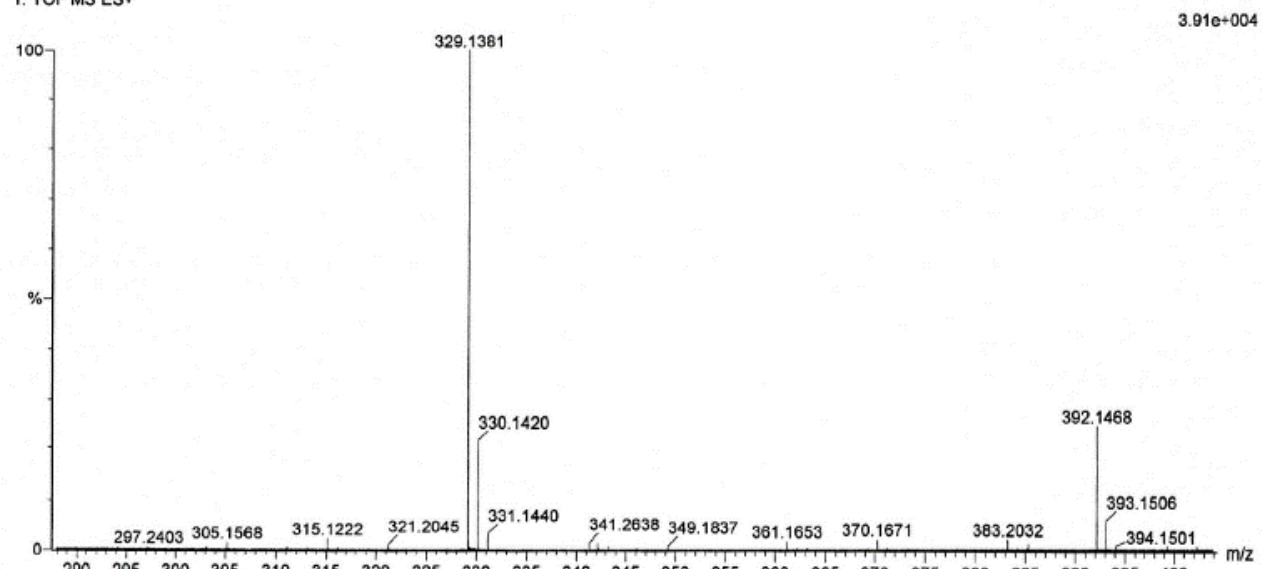

$\begin{array}{llll}\text { Minimum: } & & & -1.5 \\ \text { Maximum: } & 5.0 & 3.0 & 50.0\end{array}$

Mass Calc. Mass mDa PPM DBE

$\begin{array}{lllll}329.1381 & 329.1389 & -0.8 & -2.4 & 9.5\end{array}$

i-FIT i-FIT (Norm) Formula

$\begin{array}{lllll}526.4 & 0.0 & \text { C19 } & \text { H21 } & 05\end{array}$ 
Figure S80. IR spectrum of fortalpinoid F (6).

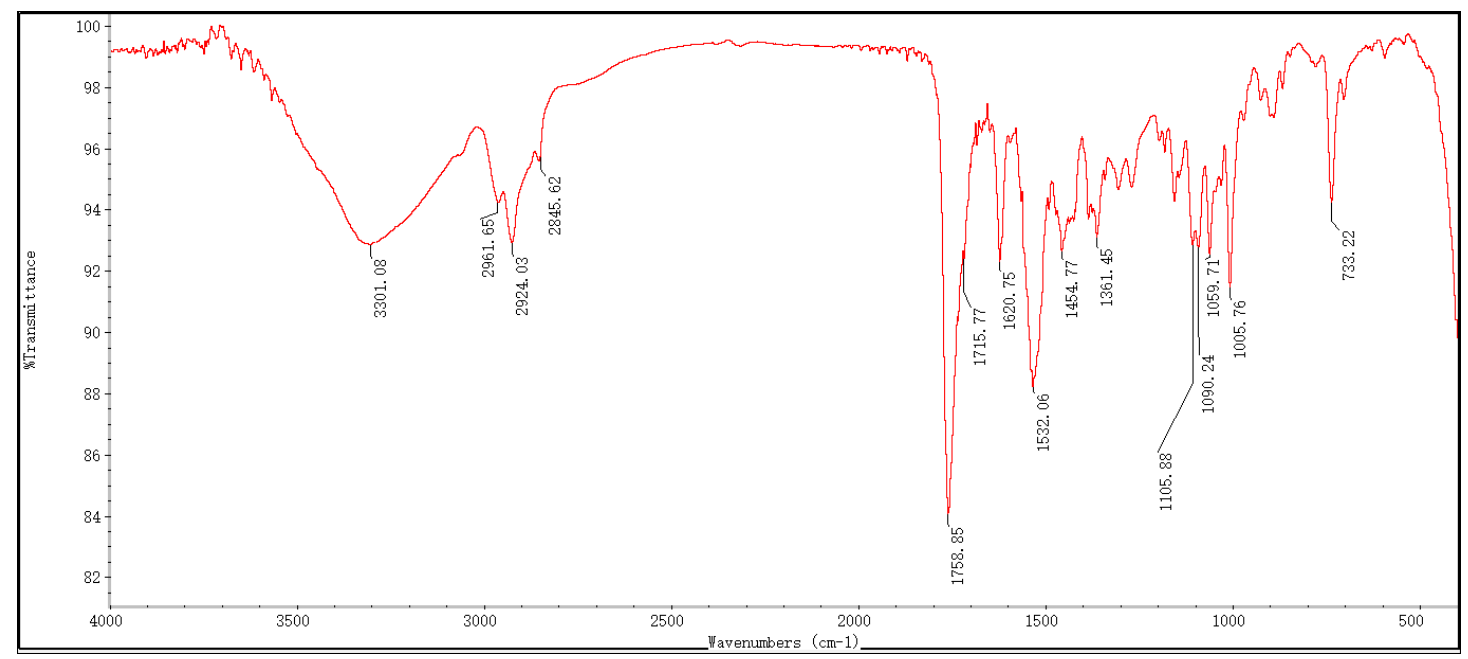

Figure S81. ${ }^{1} \mathrm{H}$ NMR spectrum of fortalpinoid G (7) in methanol- $d_{4}$.

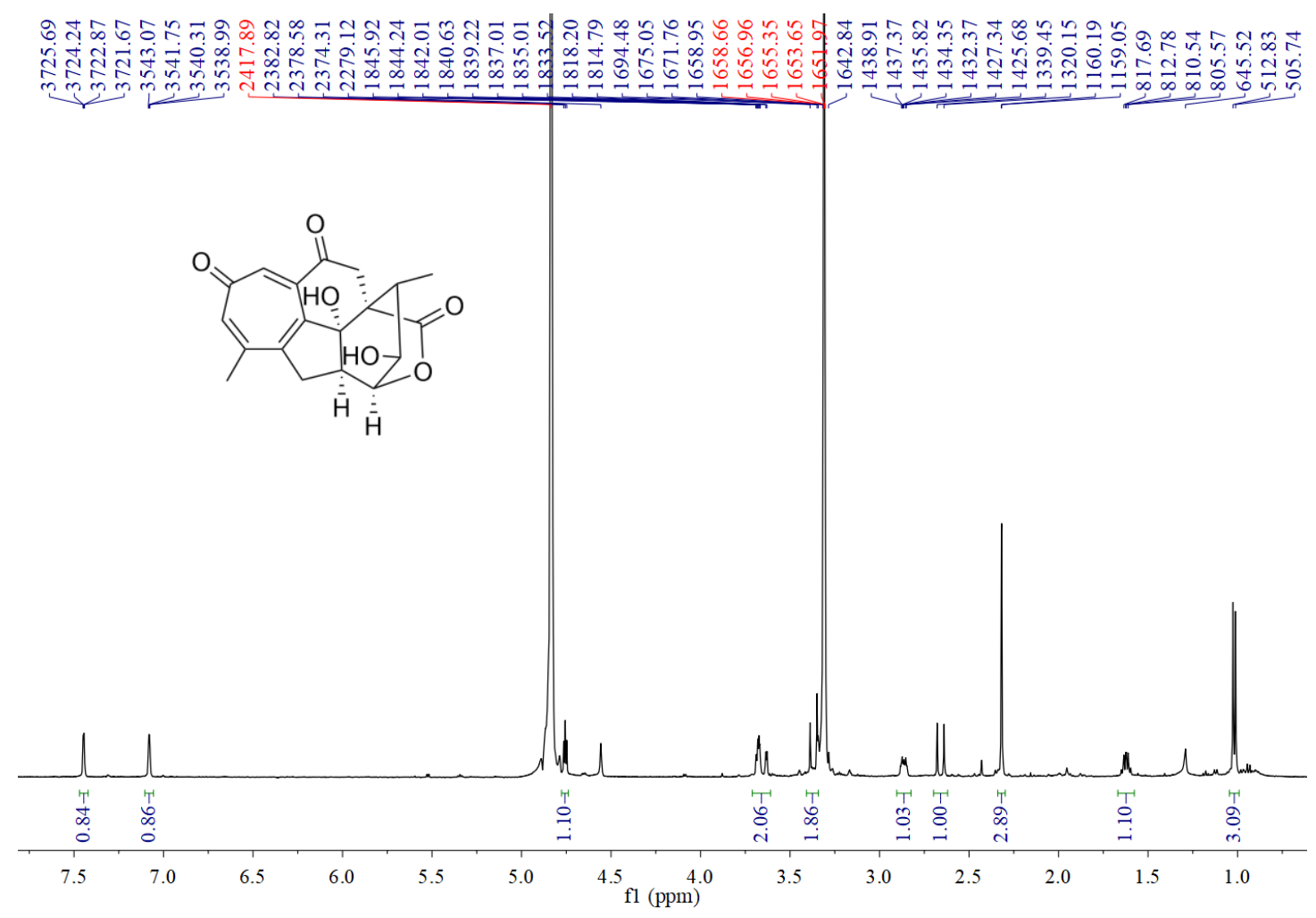


Figure S82. ${ }^{13} \mathrm{C}$ NMR spectrum of fortalpinoid G (7) in methanol- $d_{4}$.
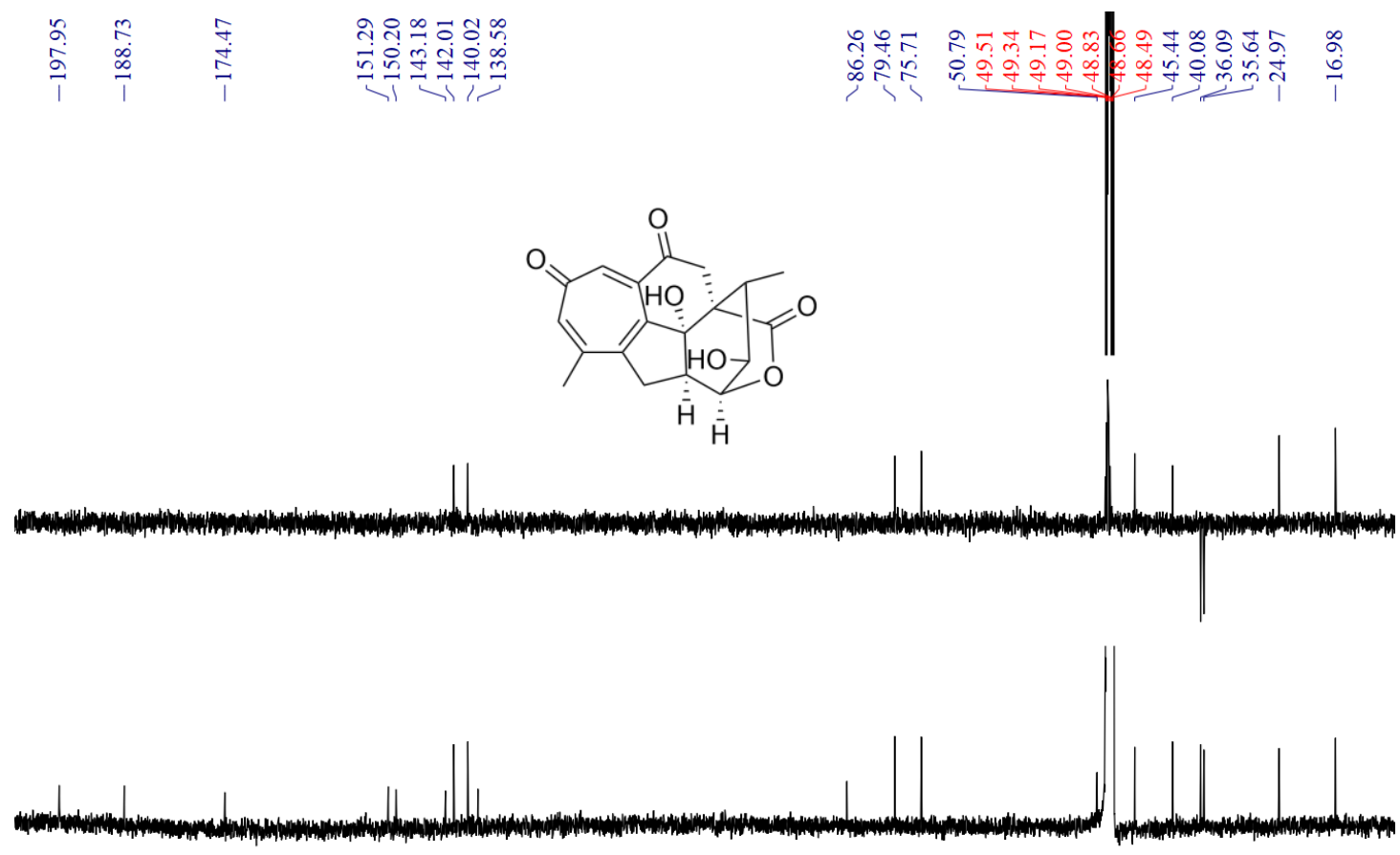

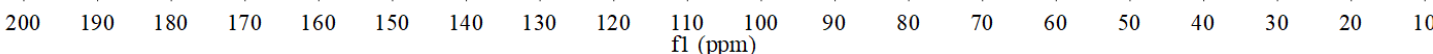

Figure S83. HSQC spectrum of fortalpinoid G (7) in methanol- $d_{4}$.

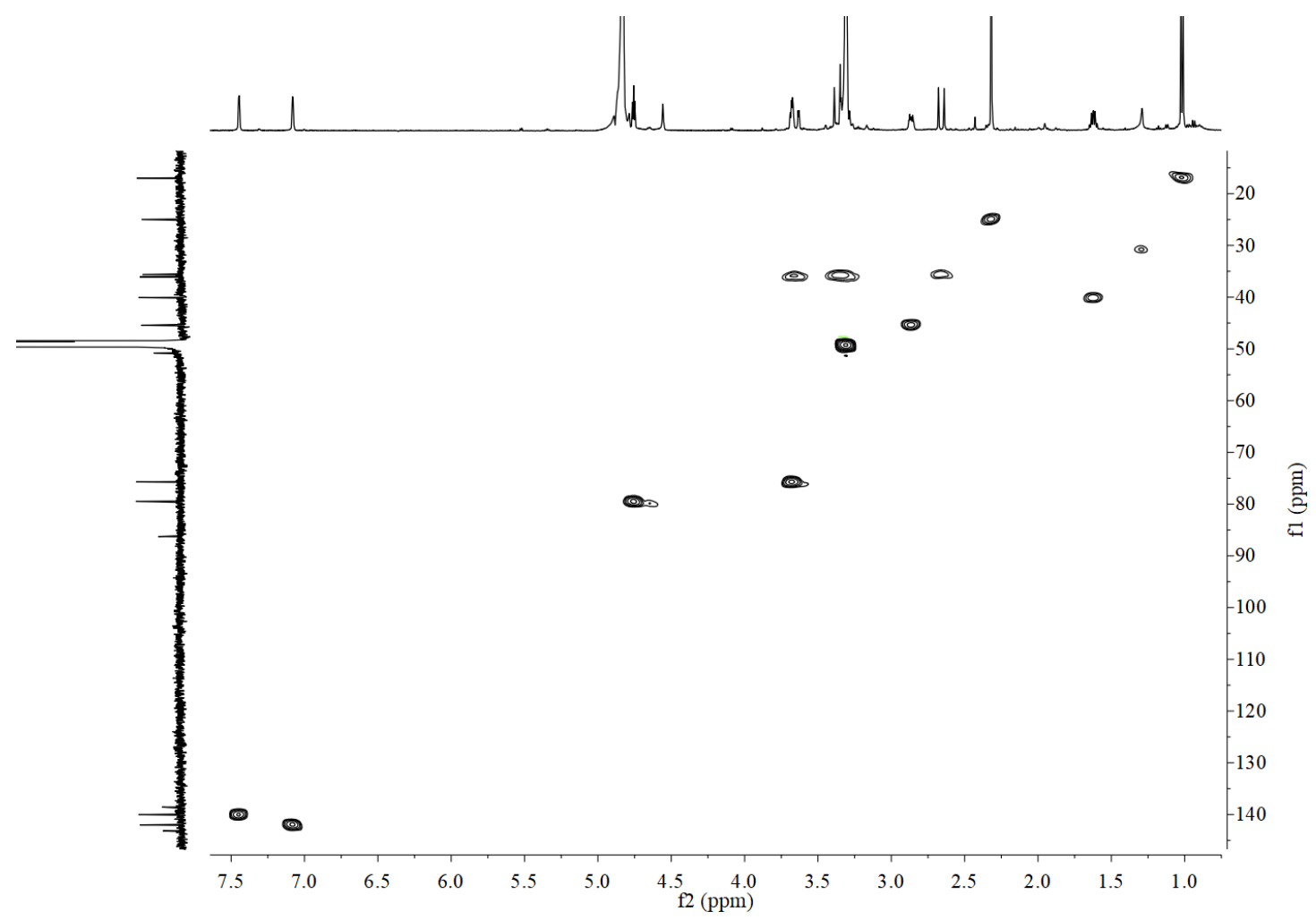


Figure S84. HMBC spectrum of fortalpinoid G (7) in methanol- $d_{4}$.

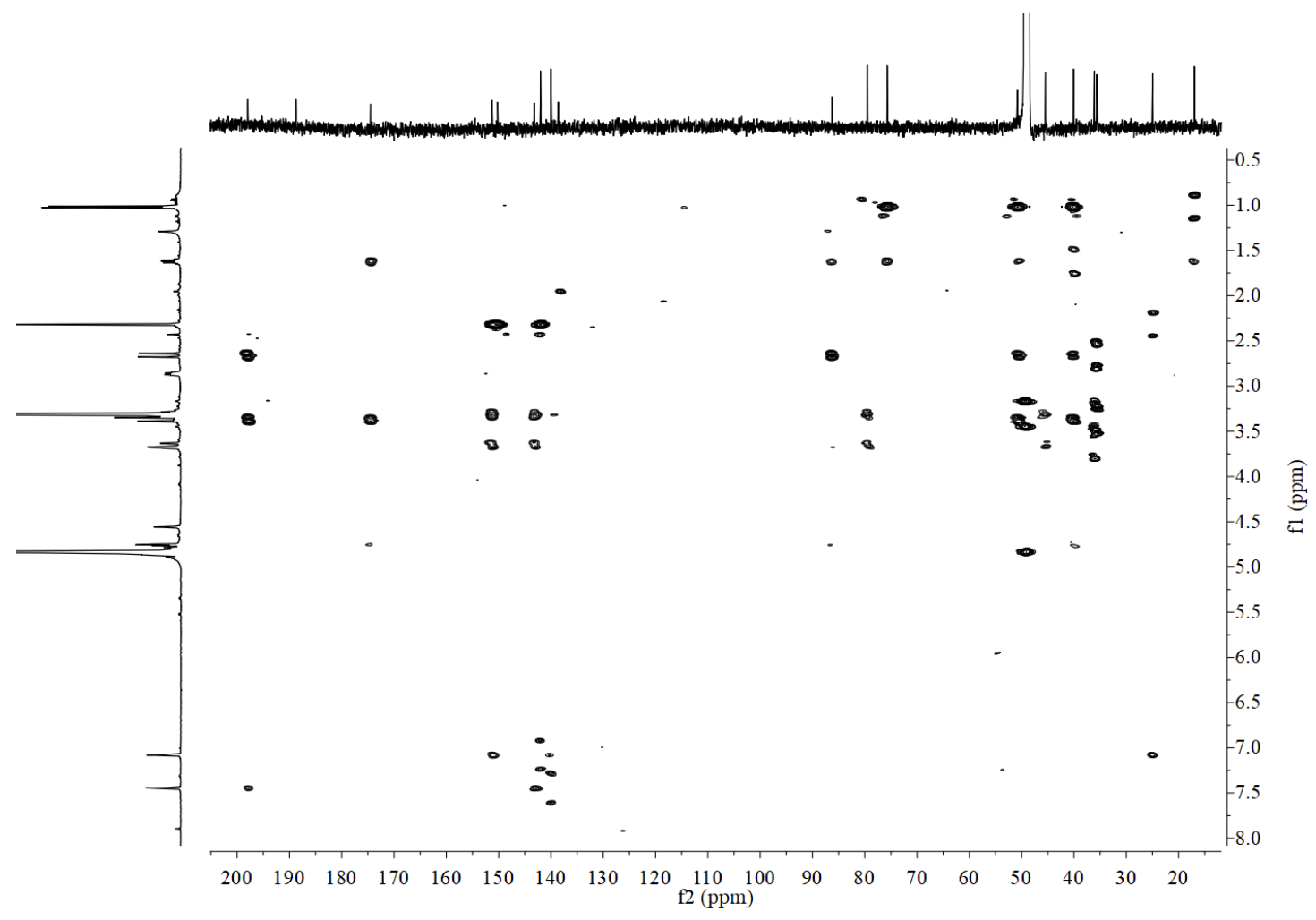

Figure S85. ${ }^{1} \mathrm{H}-{ }^{1} \mathrm{H}$ COSY spectrum of fortalpinoid G (7) in methanol- $d_{4}$.

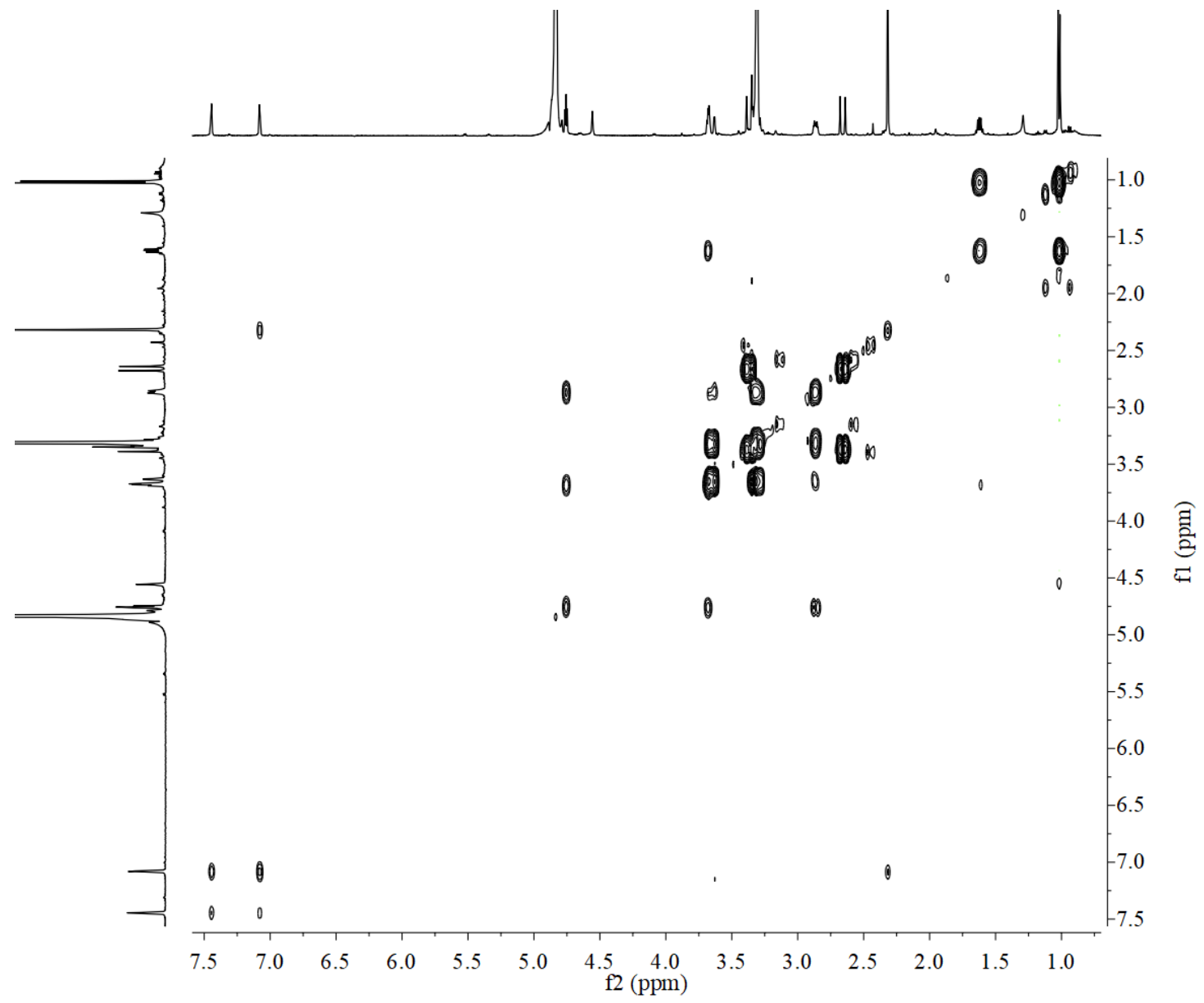


Figure S86. NOESY spectrum of fortalpinoid G (7) in methanol- $d_{4}$.

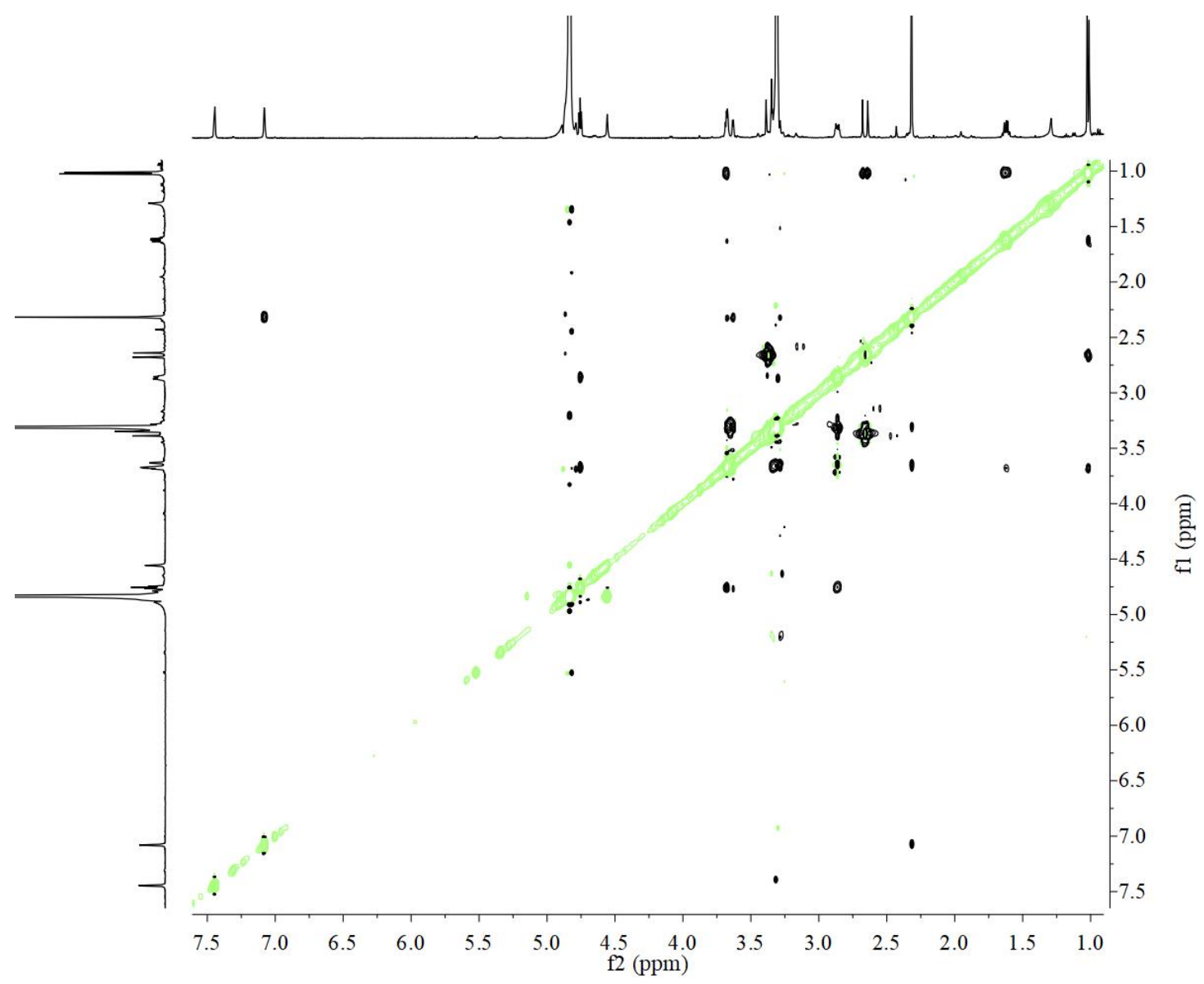

Figure S87. (+)-ESIMS spectrum of fortalpinoid G (7).

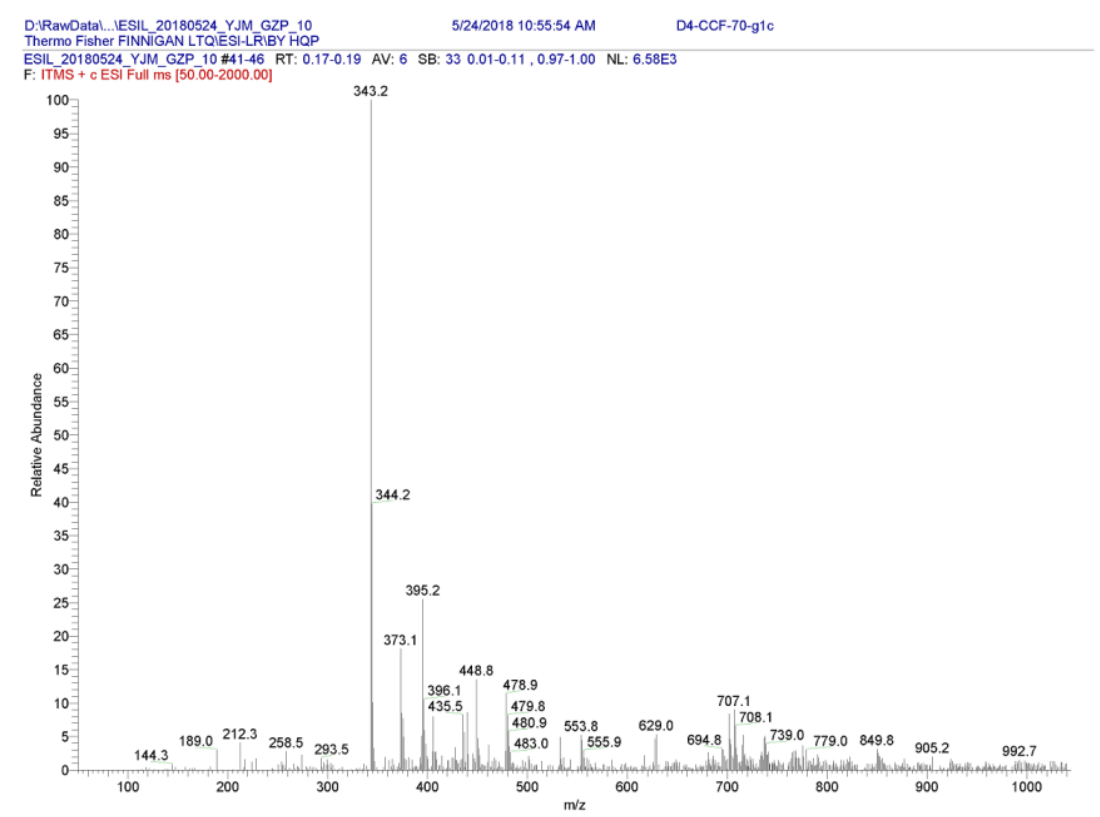


Figure S88. (-)-ESIMS spectrum of fortalpinoid G (7).

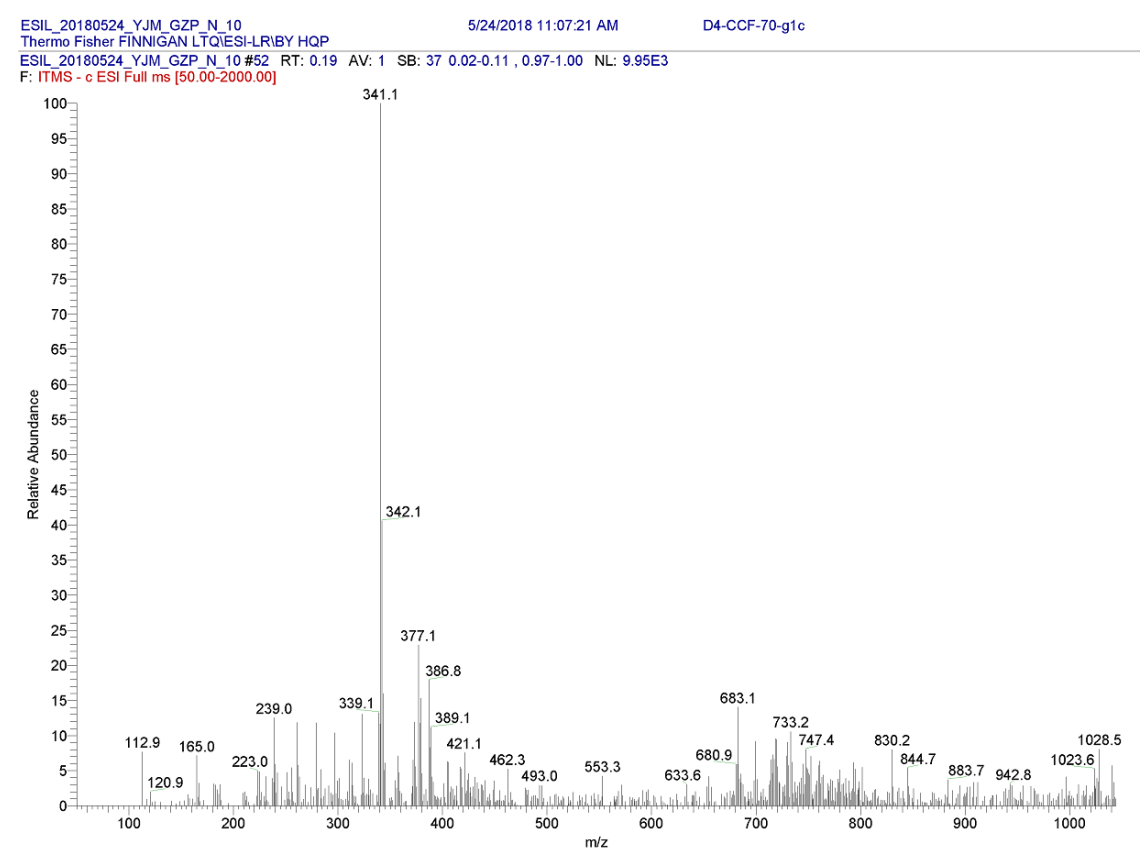

Figure S89. (-)-HRESIMS spectrum of fortalpinoid G (7).

Qualitative Analysis Report

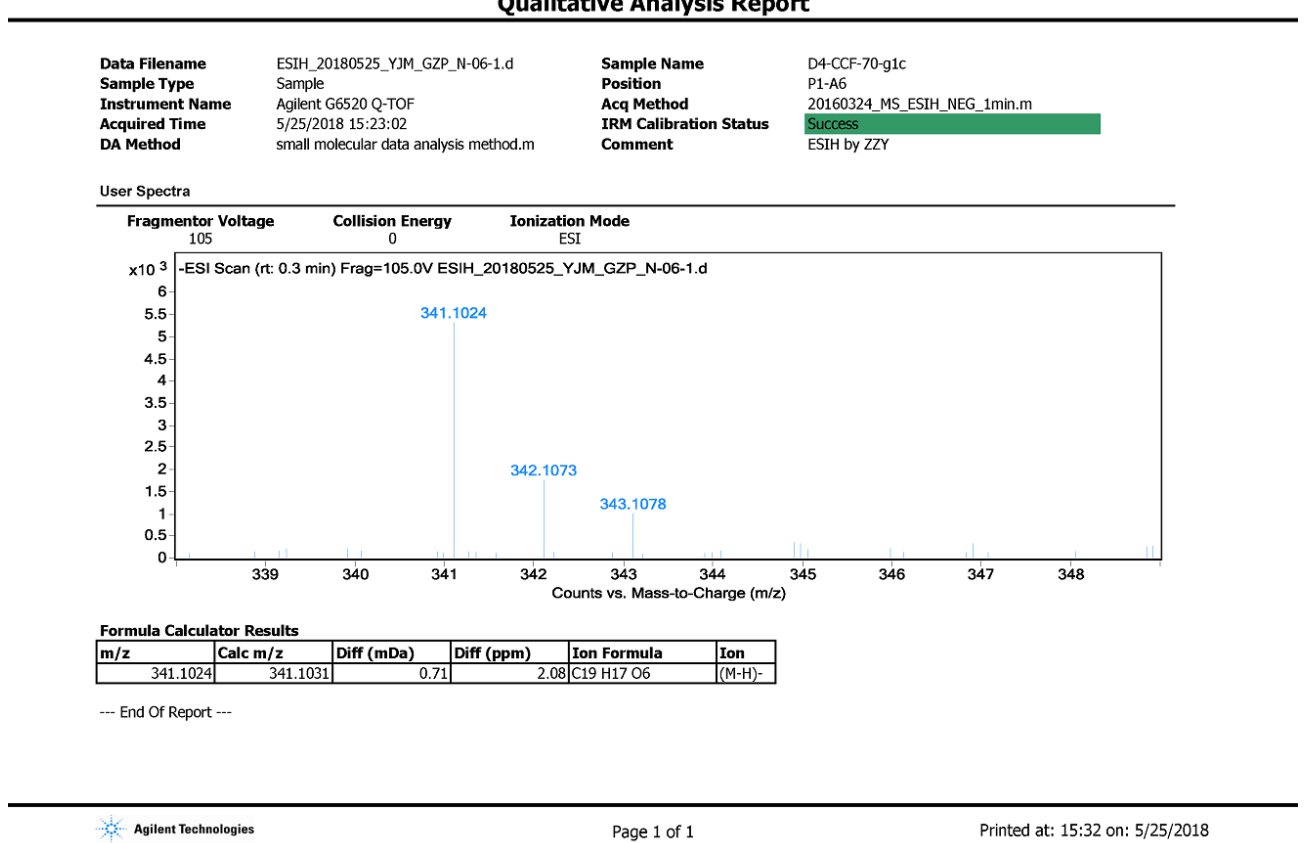


Figure S90. IR spectrum of fortalpinoid G (7).

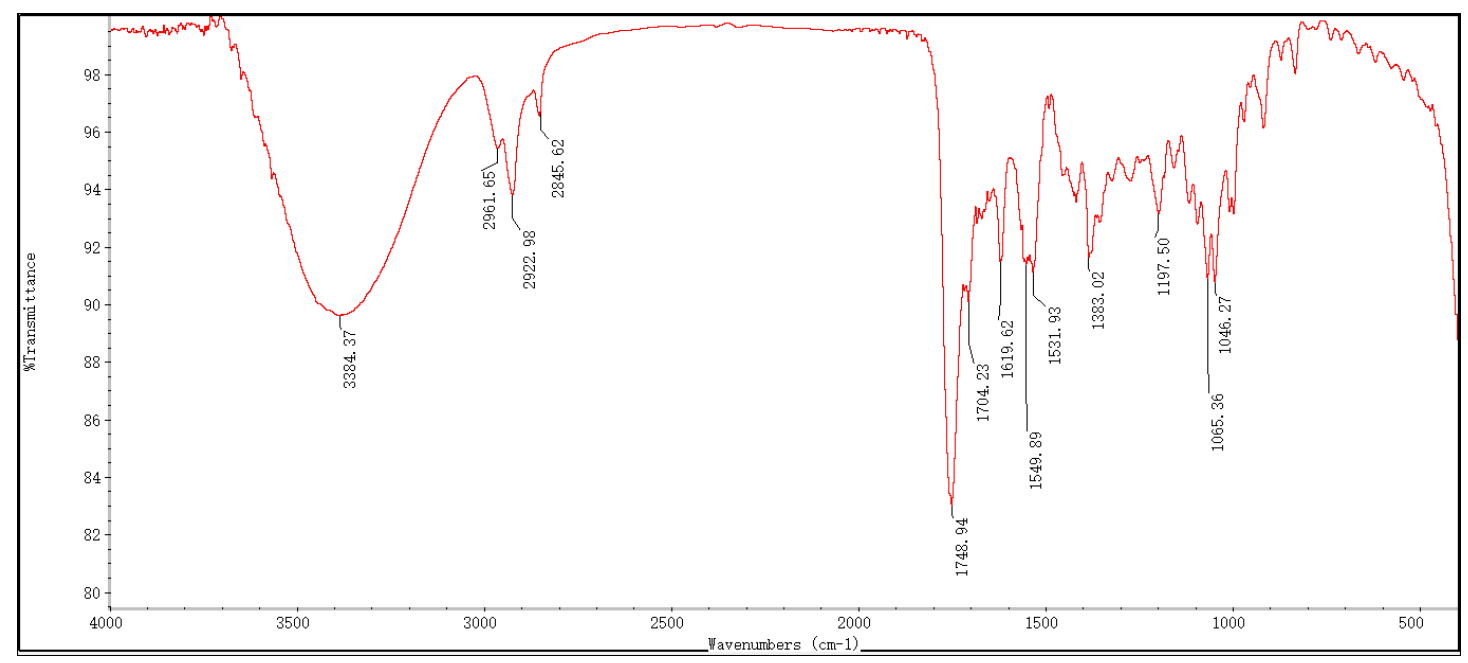

Figure S91. ${ }^{1} \mathrm{H}$ NMR spectrum of fortalpinoid $\mathrm{H}(\mathbf{8})$ in $\mathrm{CDCl}_{3}$.

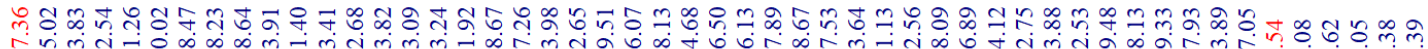

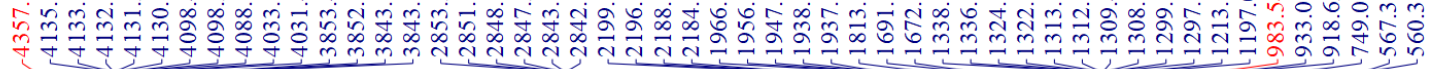

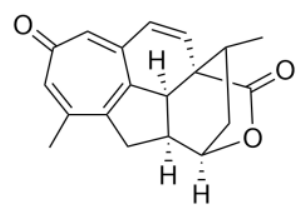

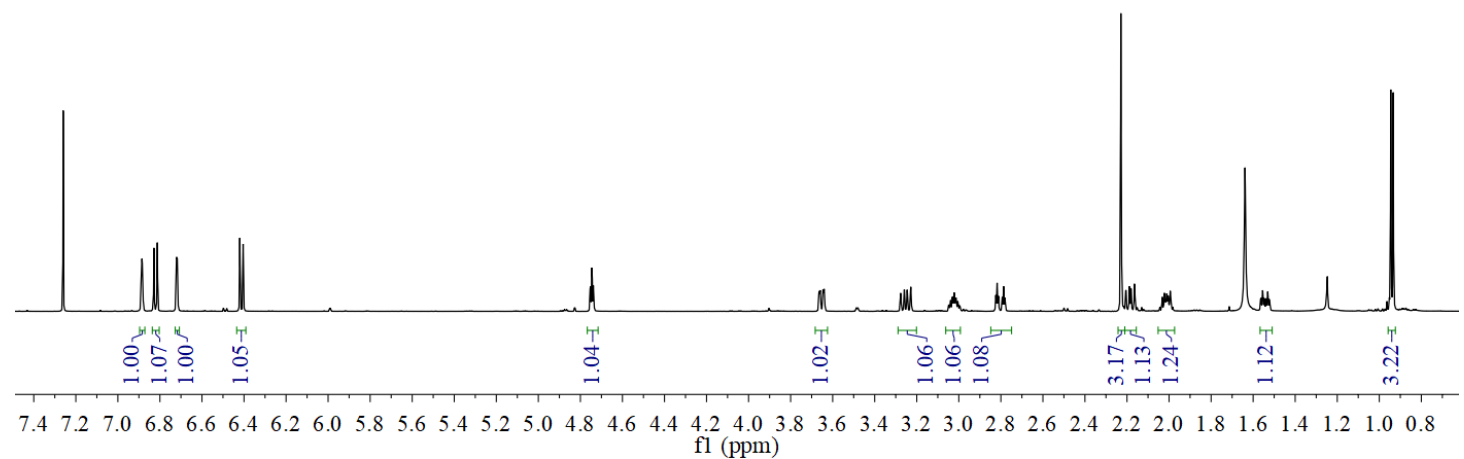


Figure S92. ${ }^{13} \mathrm{C}$ NMR spectrum of fortalpinoid $\mathrm{H}(\mathbf{8})$ in $\mathrm{CDCl}_{3}$.
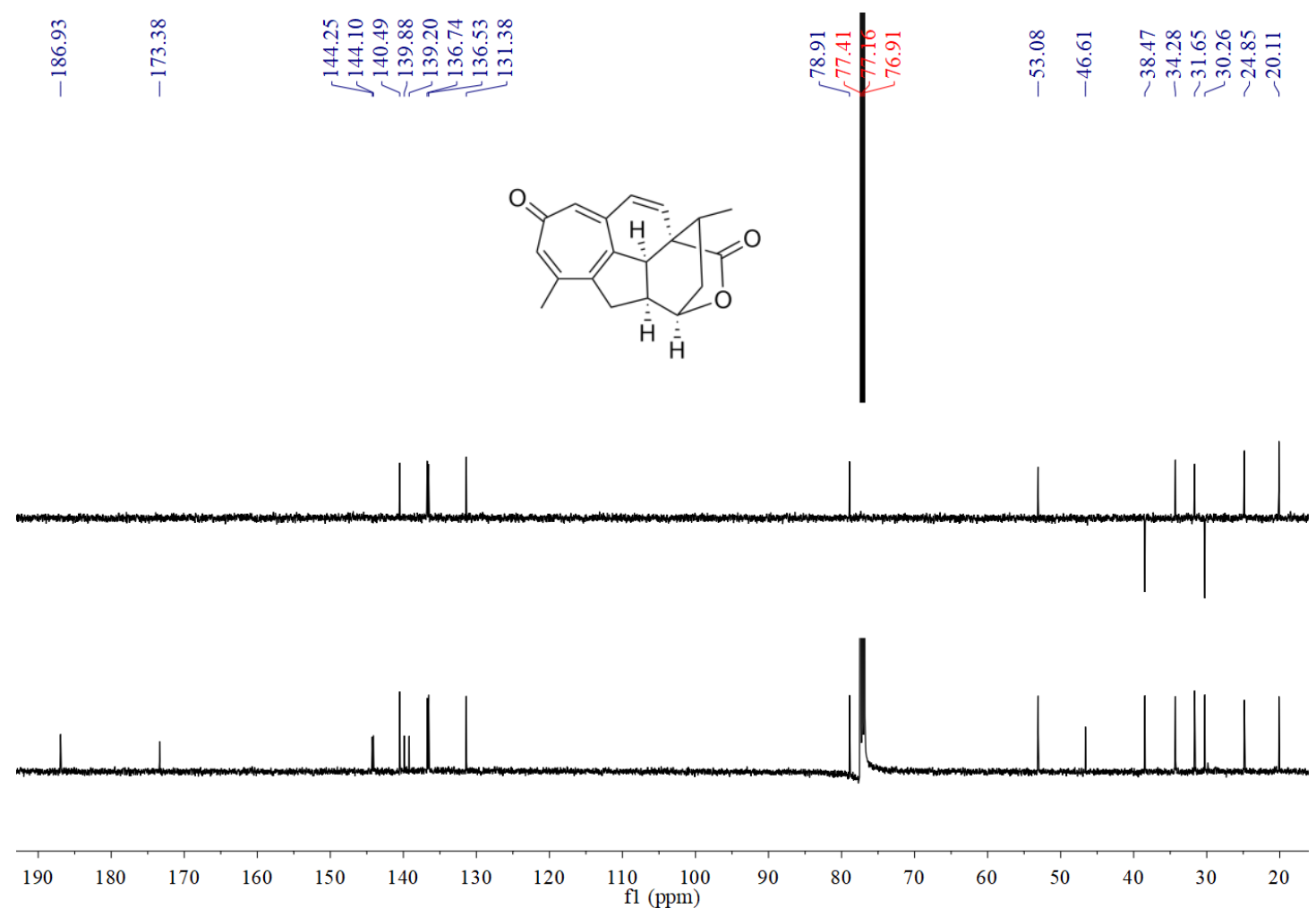

Figure S93. HSQC spectrum of fortalpinoid $\mathrm{H}(\mathbf{8})$ in $\mathrm{CDCl}_{3}$.

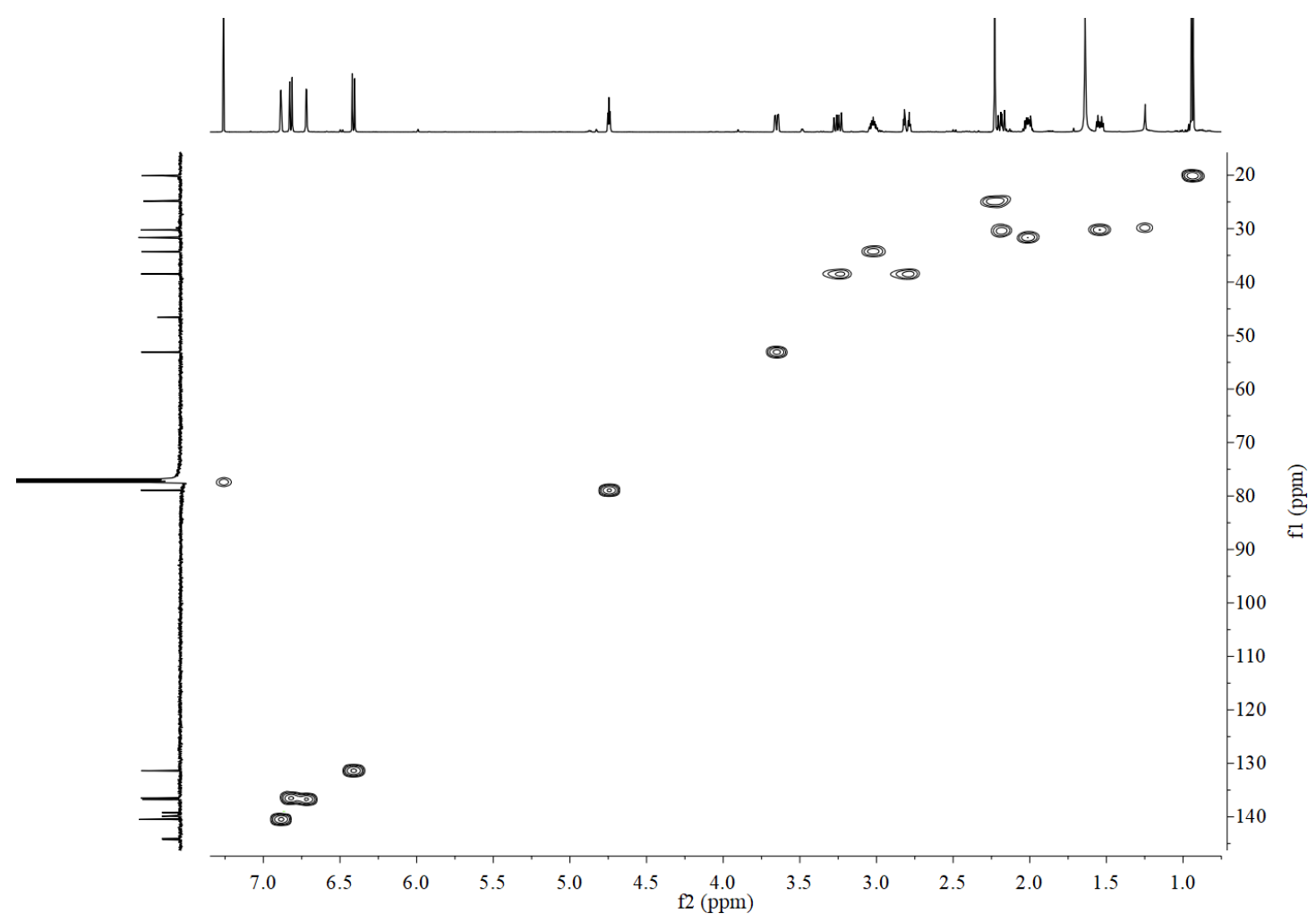


Figure S94. HMBC spectrum of fortalpinoid $\mathrm{H}(8)$ in $\mathrm{CDCl}_{3}$.

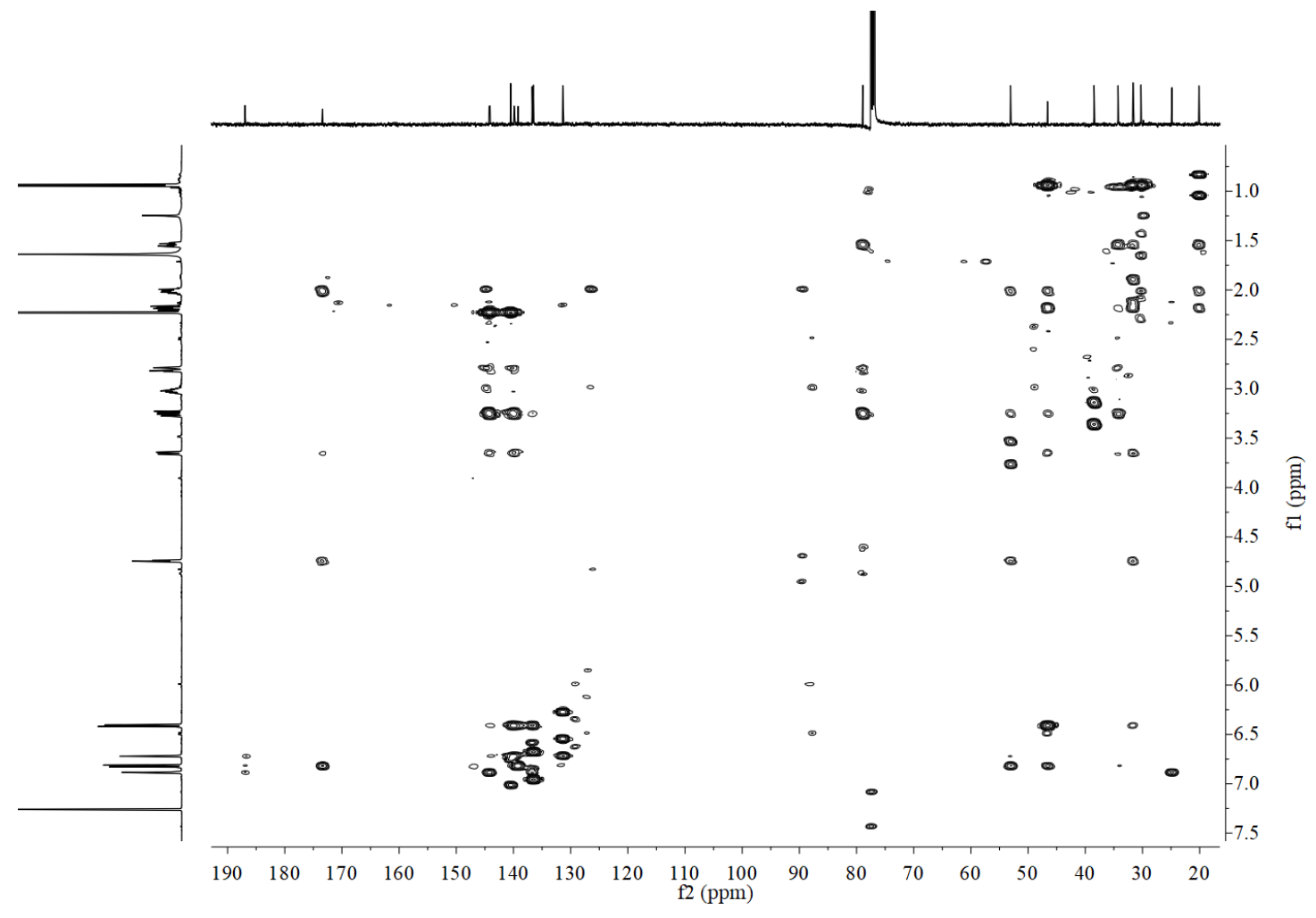

Figure S95. ${ }^{1} \mathrm{H}-{ }^{1} \mathrm{H}$ COSY spectrum of fortalpinoid $\mathrm{H}(8)$ in $\mathrm{CDCl}_{3}$.

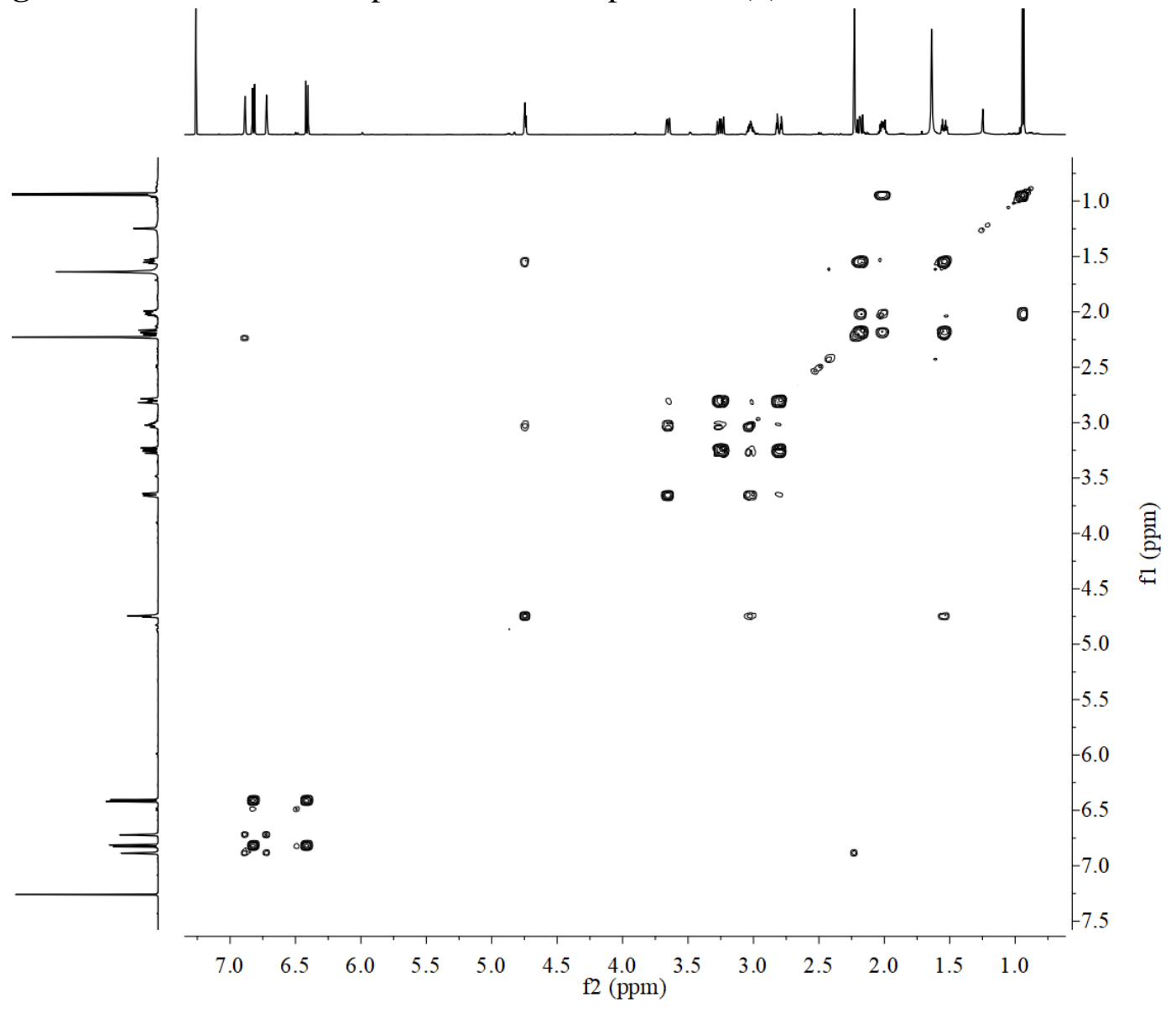


Figure S96. NOESY spectrum of fortalpinoid $\mathrm{H}(\mathbf{8})$ in $\mathrm{CDCl}_{3}$.

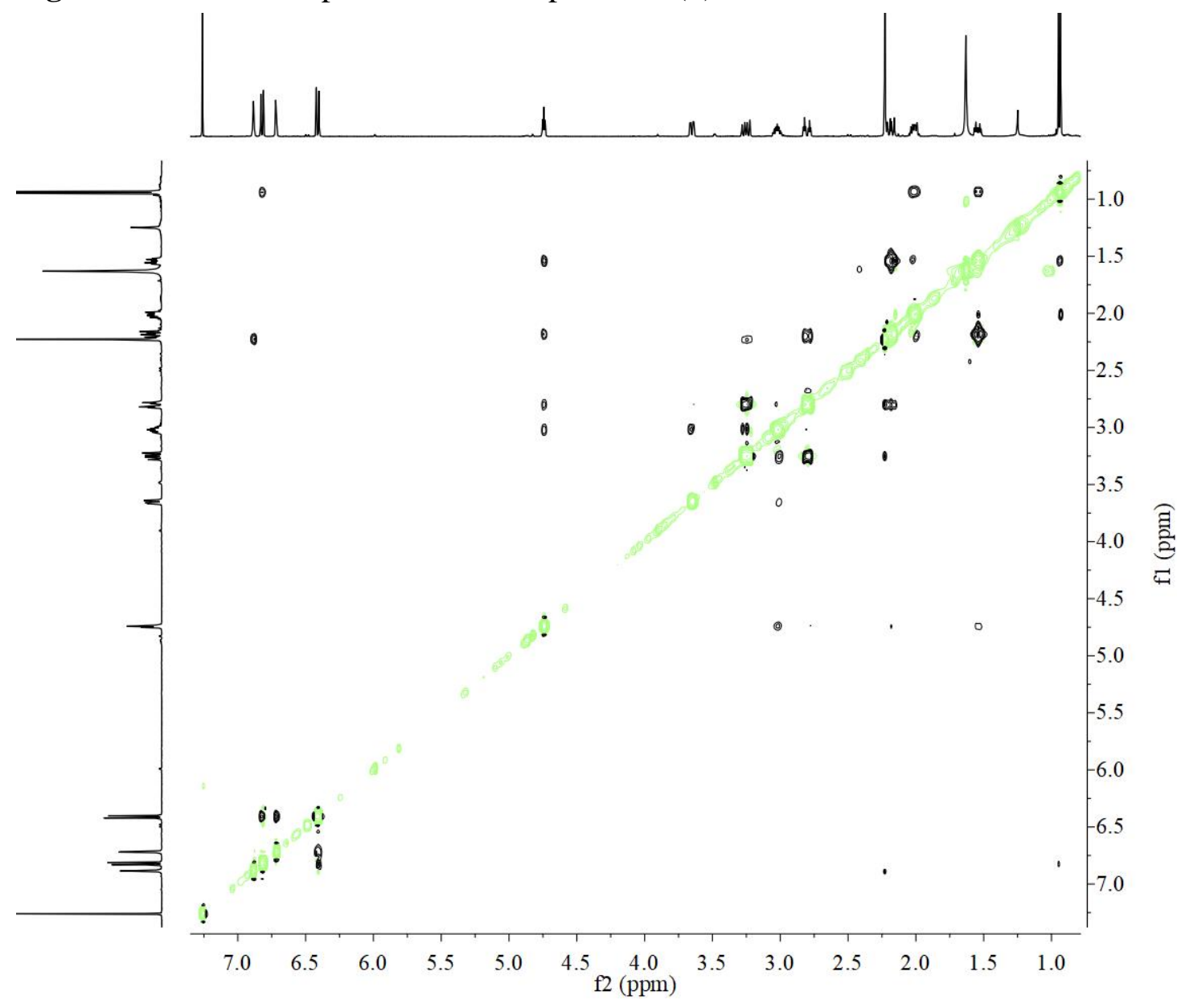

Figure S97. (+)-ESIMS spectrum of fortalpinoid H (8).

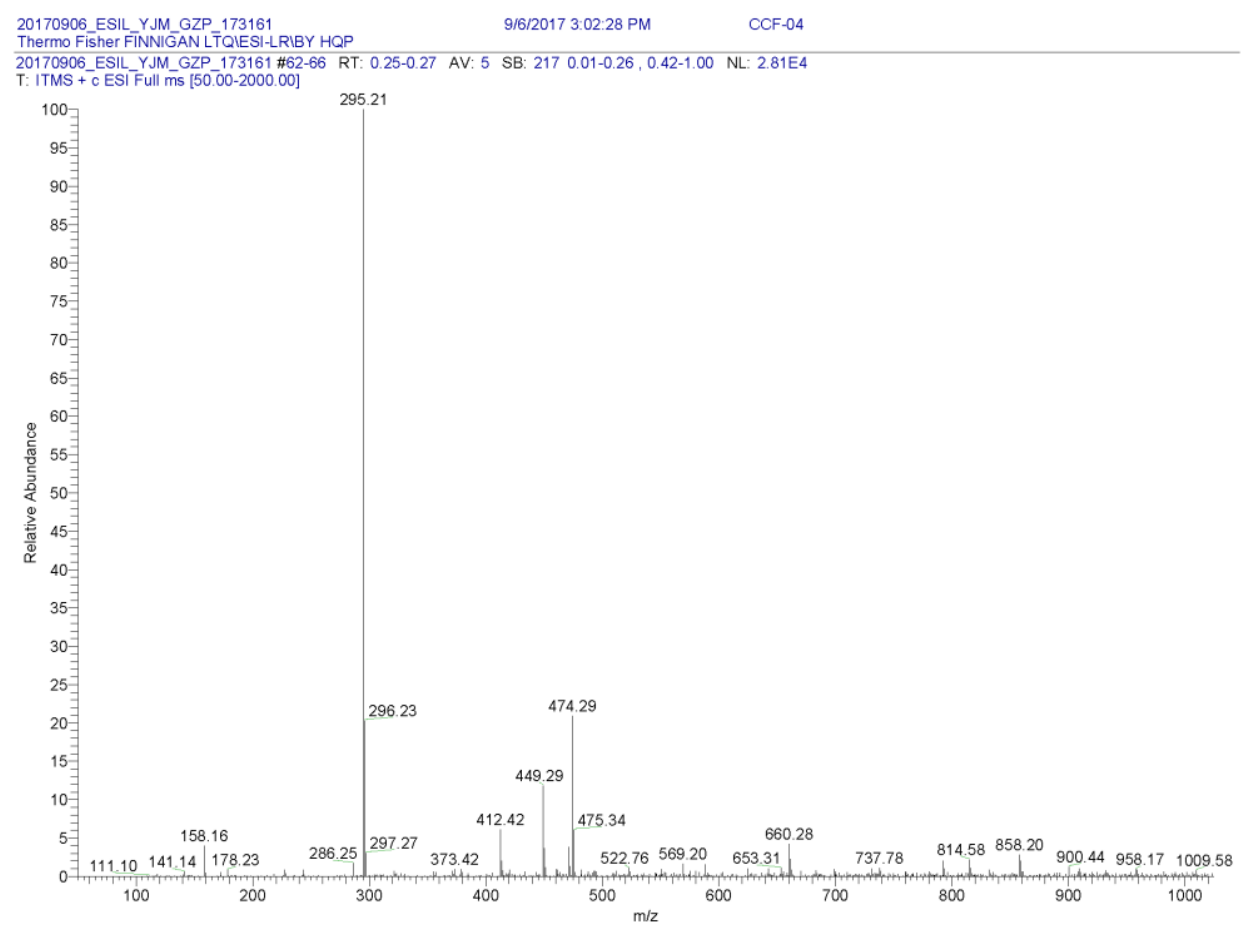


Figure S98. (+)-HRESIMS spectrum of fortalpinoid H (8).

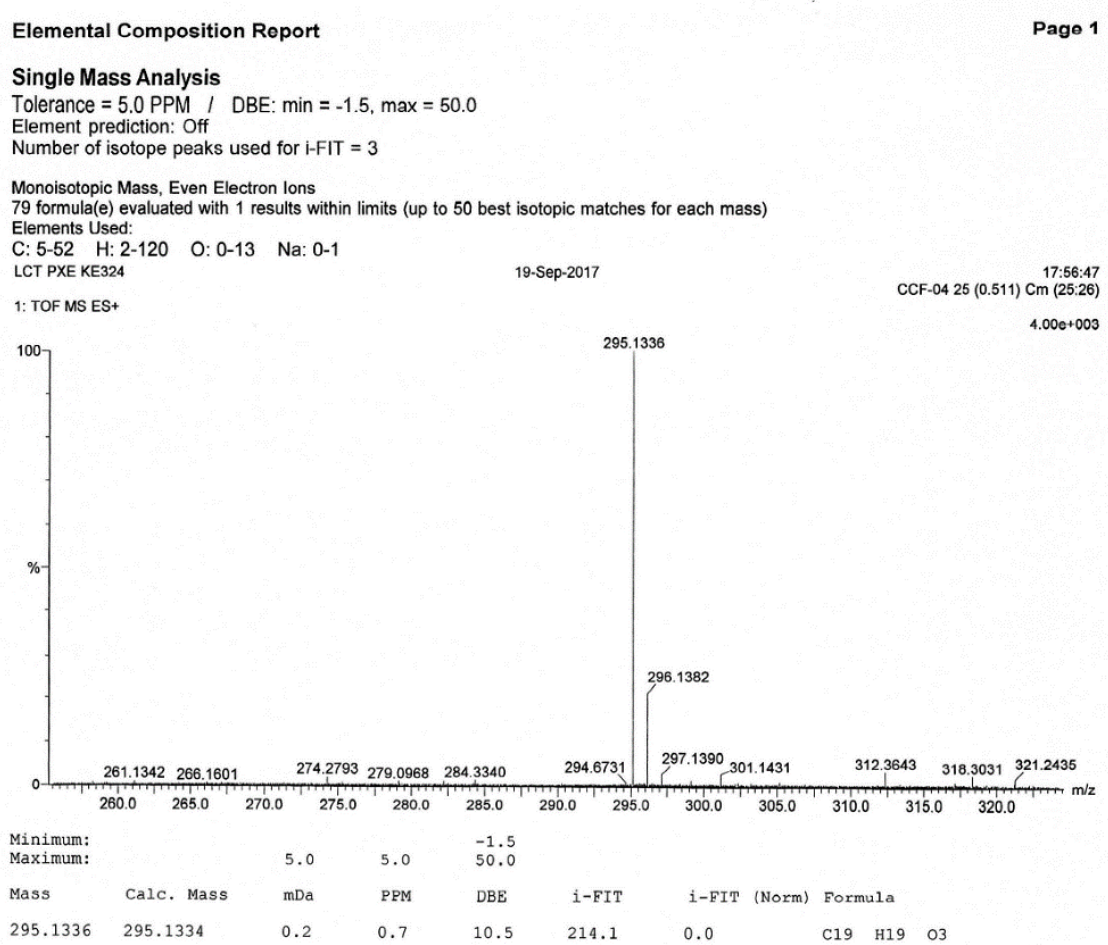

Figure S99. IR spectrum of fortalpinoid H (8).

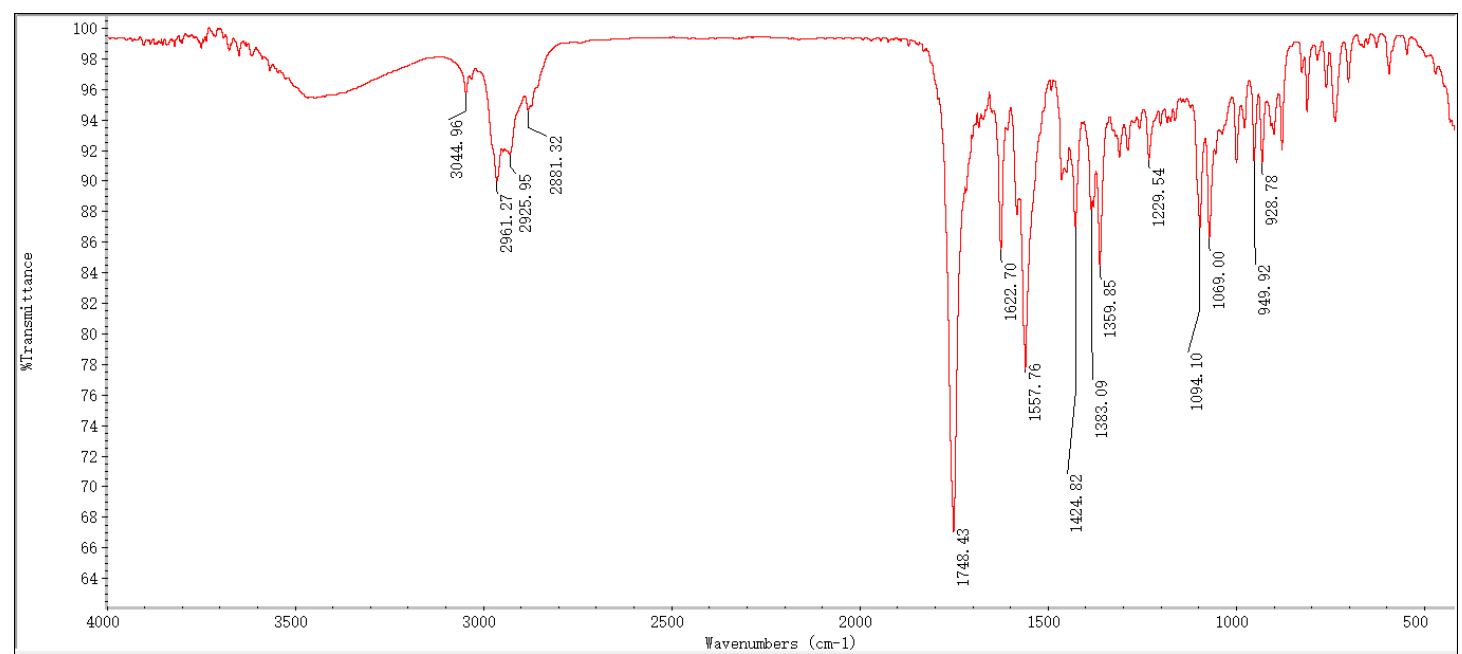


Figure S100. ${ }^{1} \mathrm{H}$ NMR spectrum of fortalpinoid I (9) in methanol- $d_{4}$.

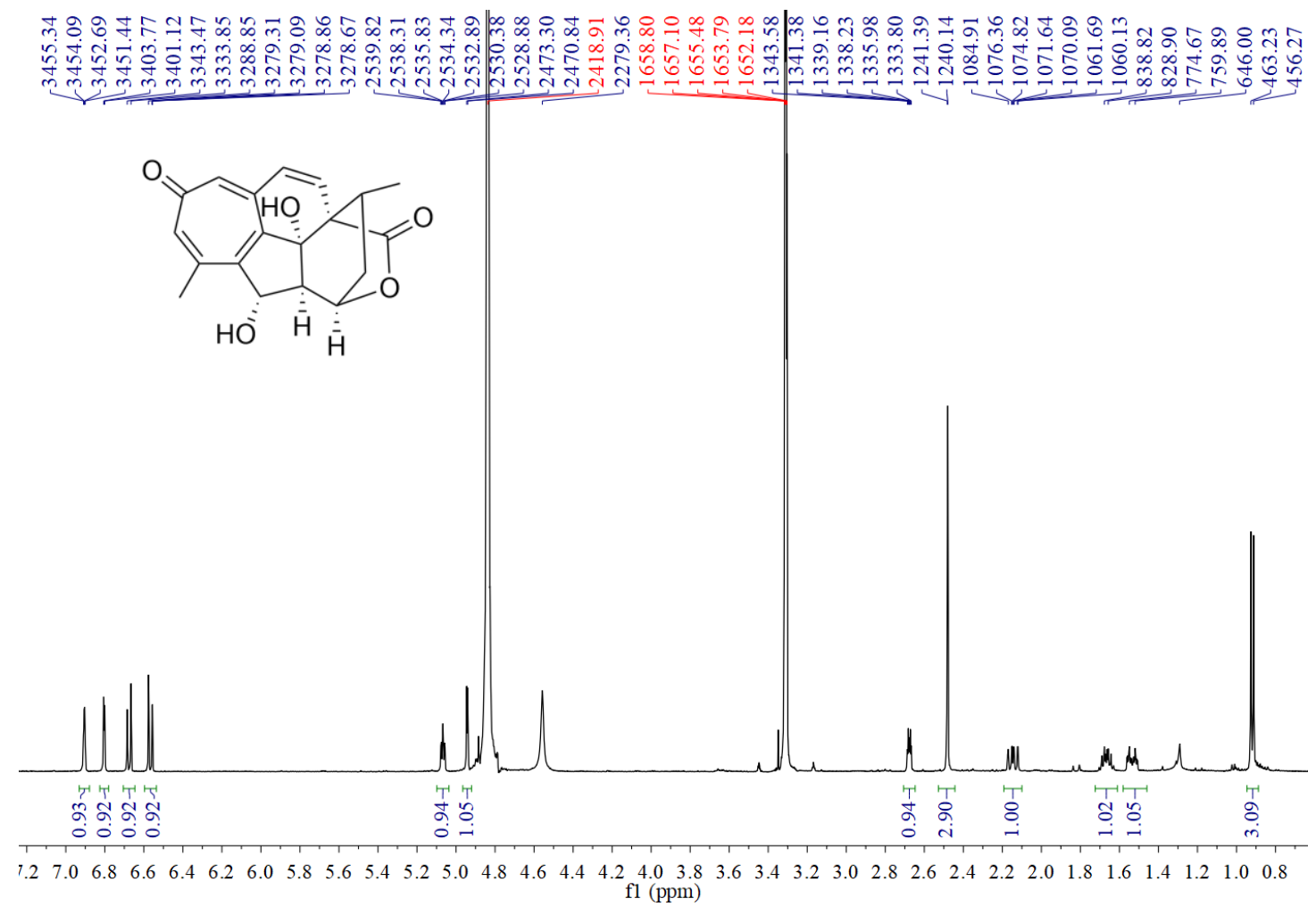

Figure S101. ${ }^{13} \mathrm{C}$ NMR spectrum of fortalpinoid I (9) in methanol- $d_{4}$.
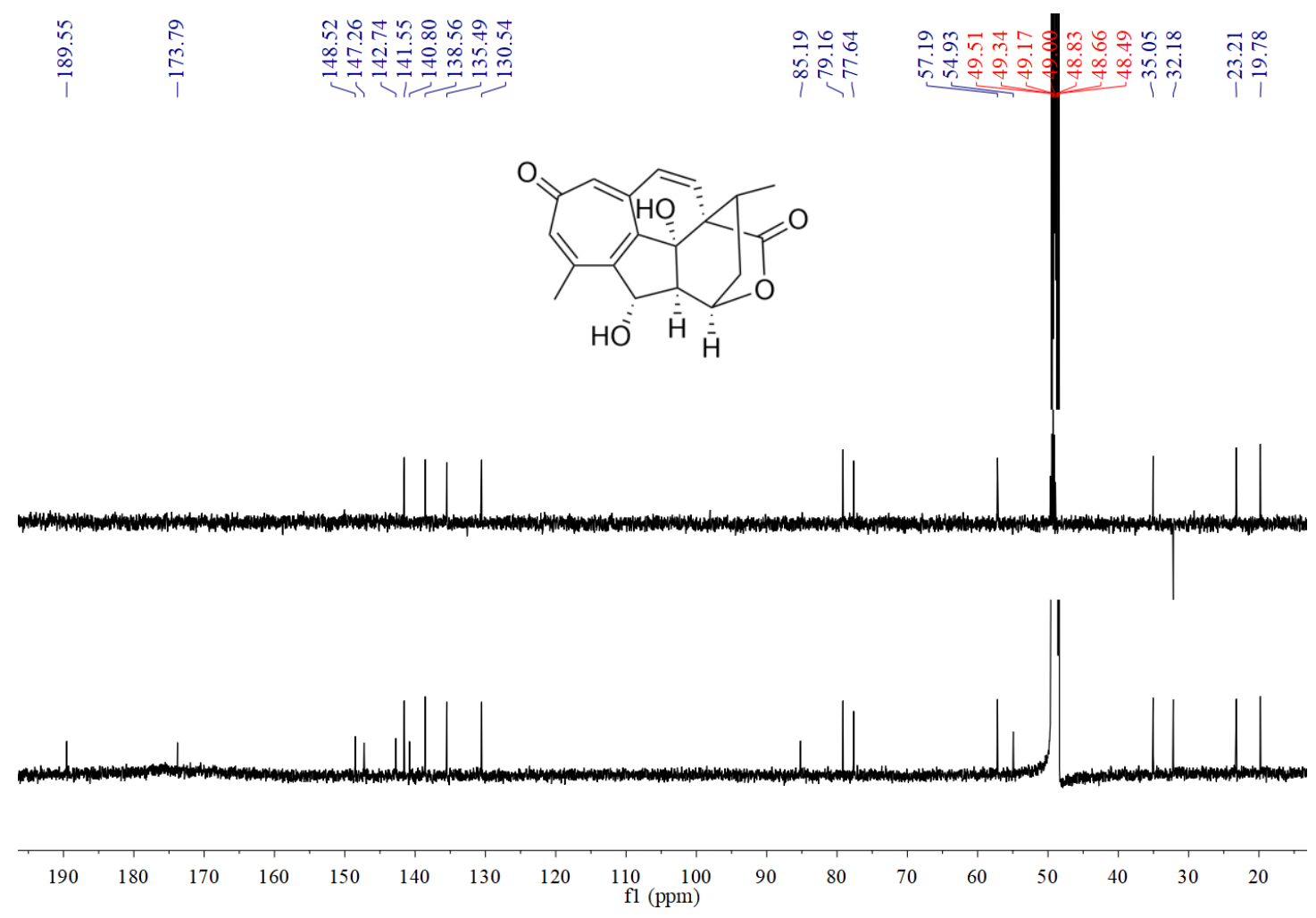
Figure S102. HSQC spectrum of fortalpinoid I (9) in methanol- $d_{4}$.

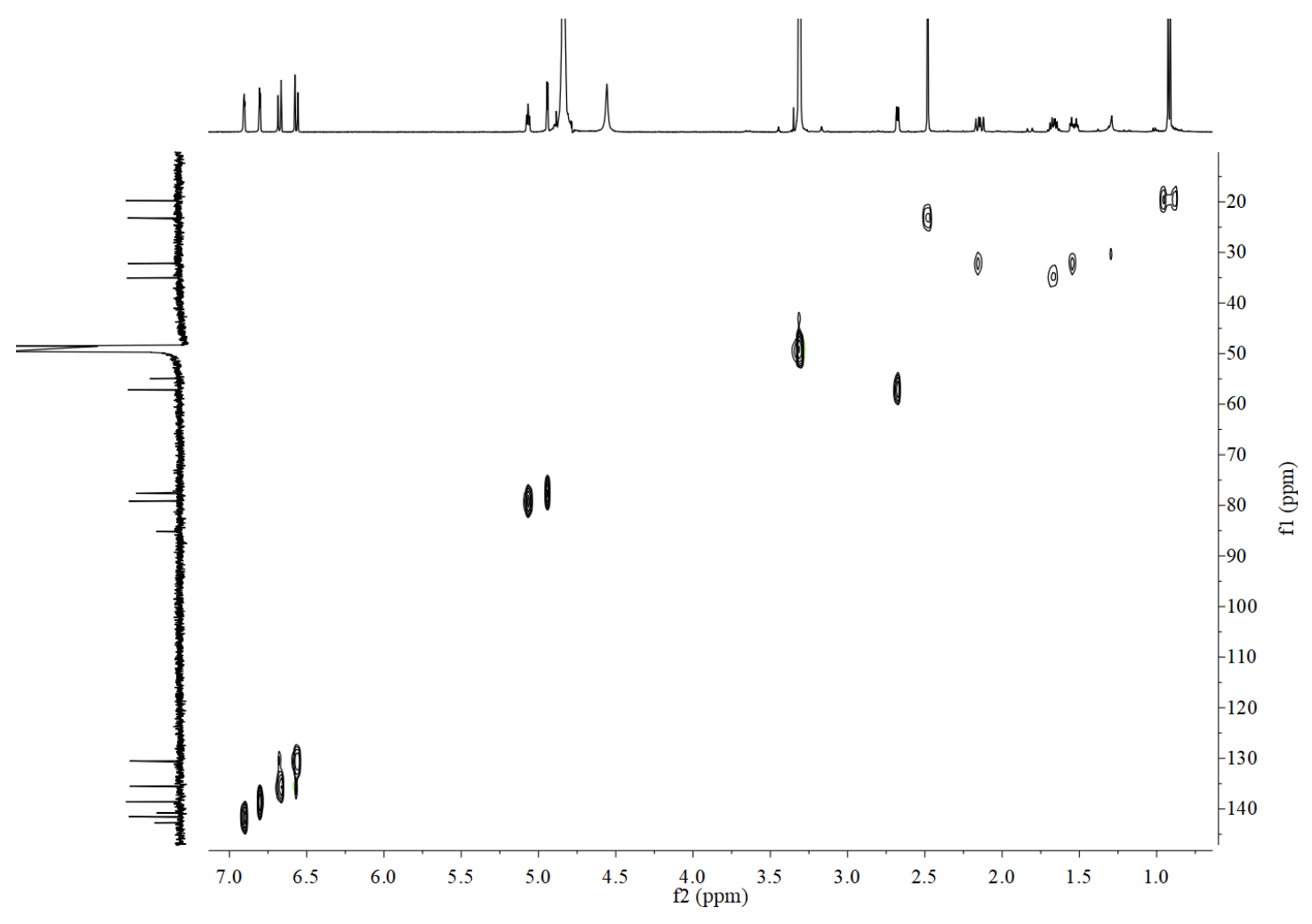

Figure S103. HMBC spectrum of fortalpinoid I (9) in methanol- $d_{4}$.

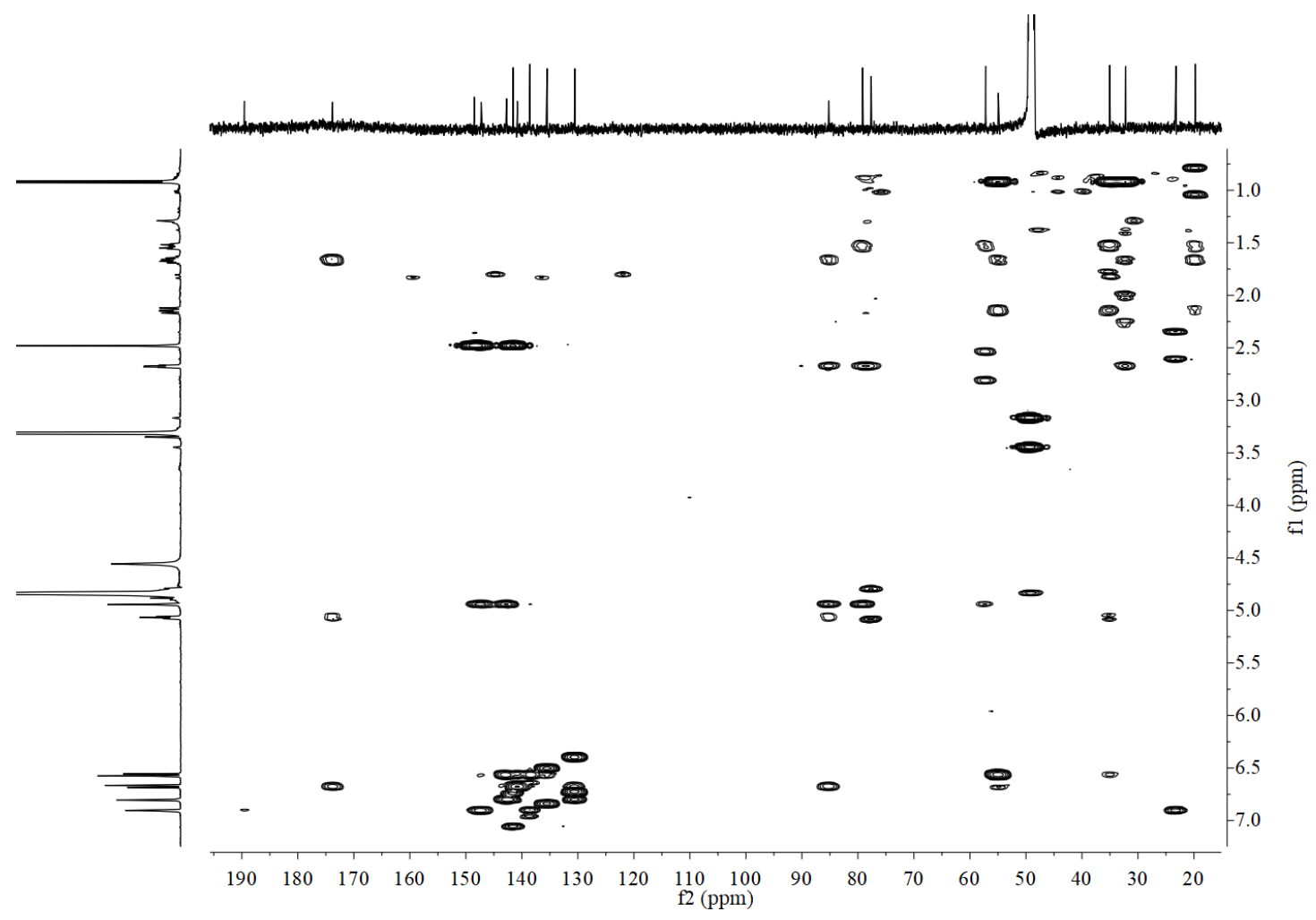


Figure S104. ${ }^{1} \mathrm{H}-{ }^{1} \mathrm{H}$ COSY spectrum of fortalpinoid I (9) in methanol- $d_{4}$.

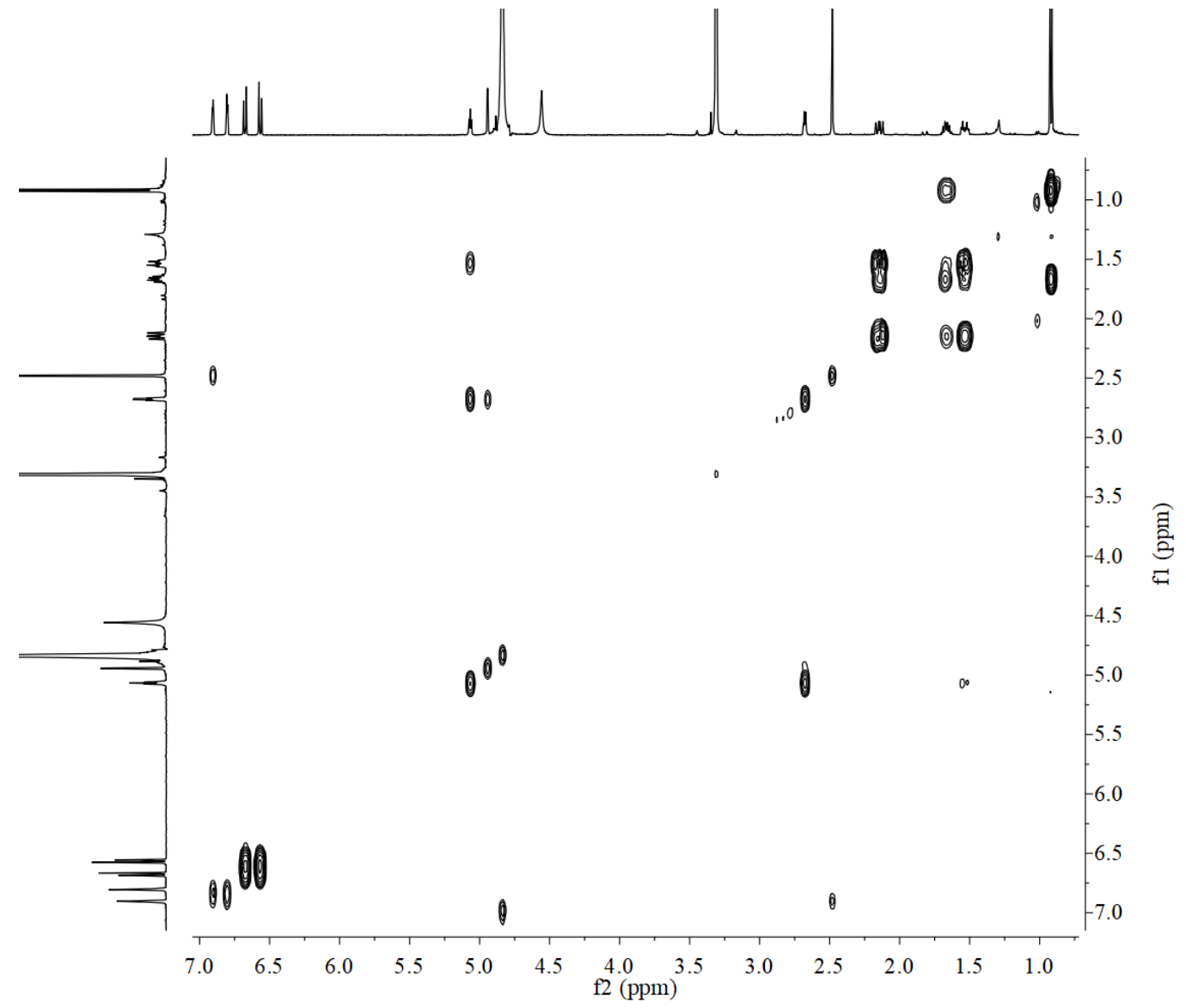

Figure S105. NOESY spectrum of fortalpinoid I (9) in methanol- $d_{4}$.

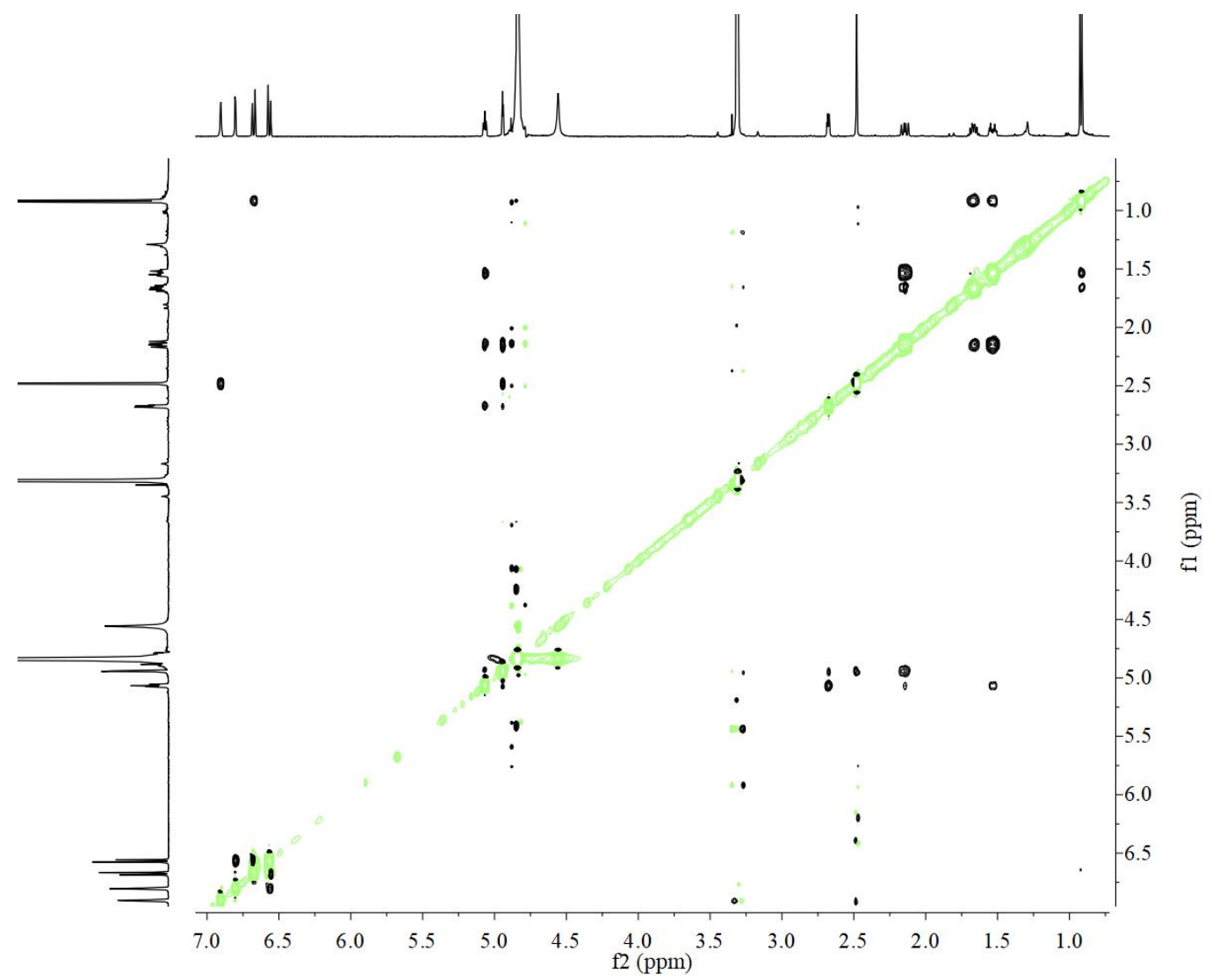


Figure S106. (+)-ESIMS spectrum of fortalpinoid I (9).

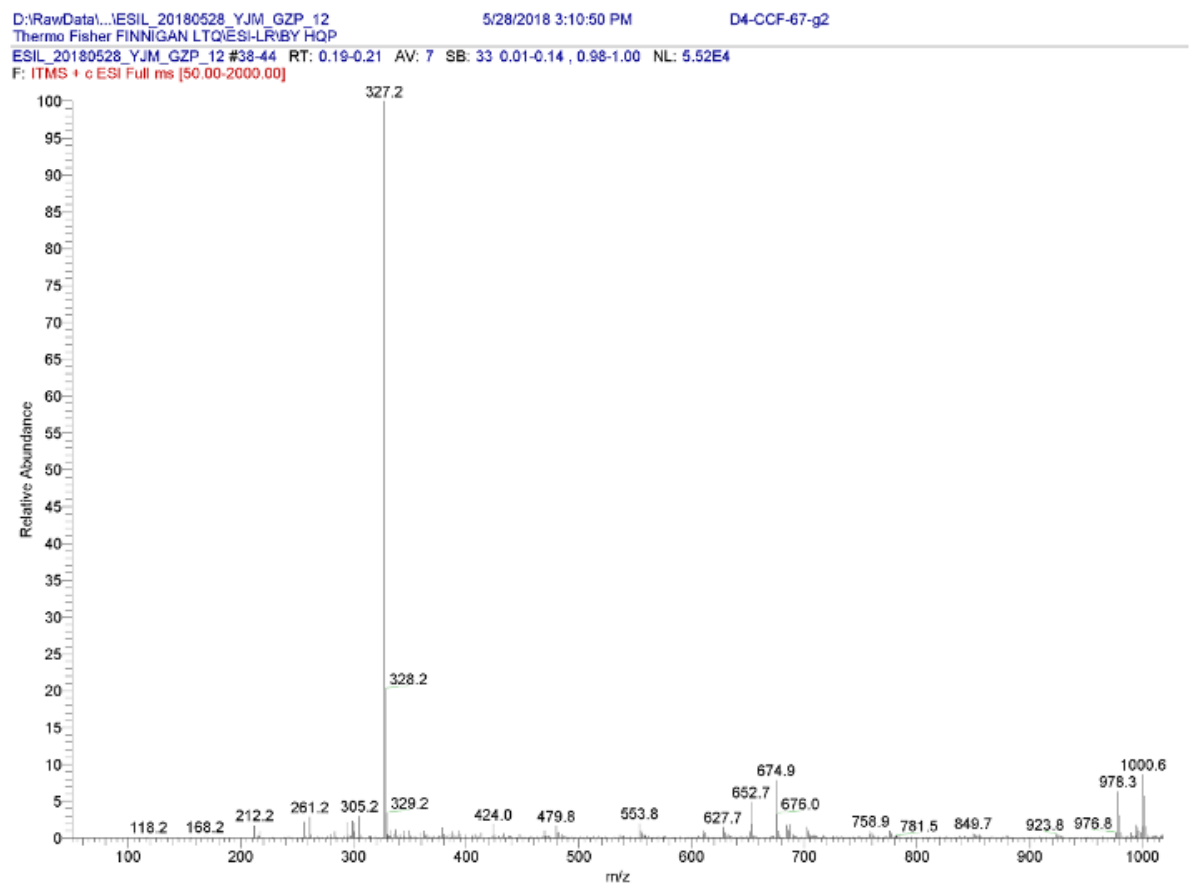

Figure S107. (-)-ESIMS spectrum of fortalpinoid I (9).

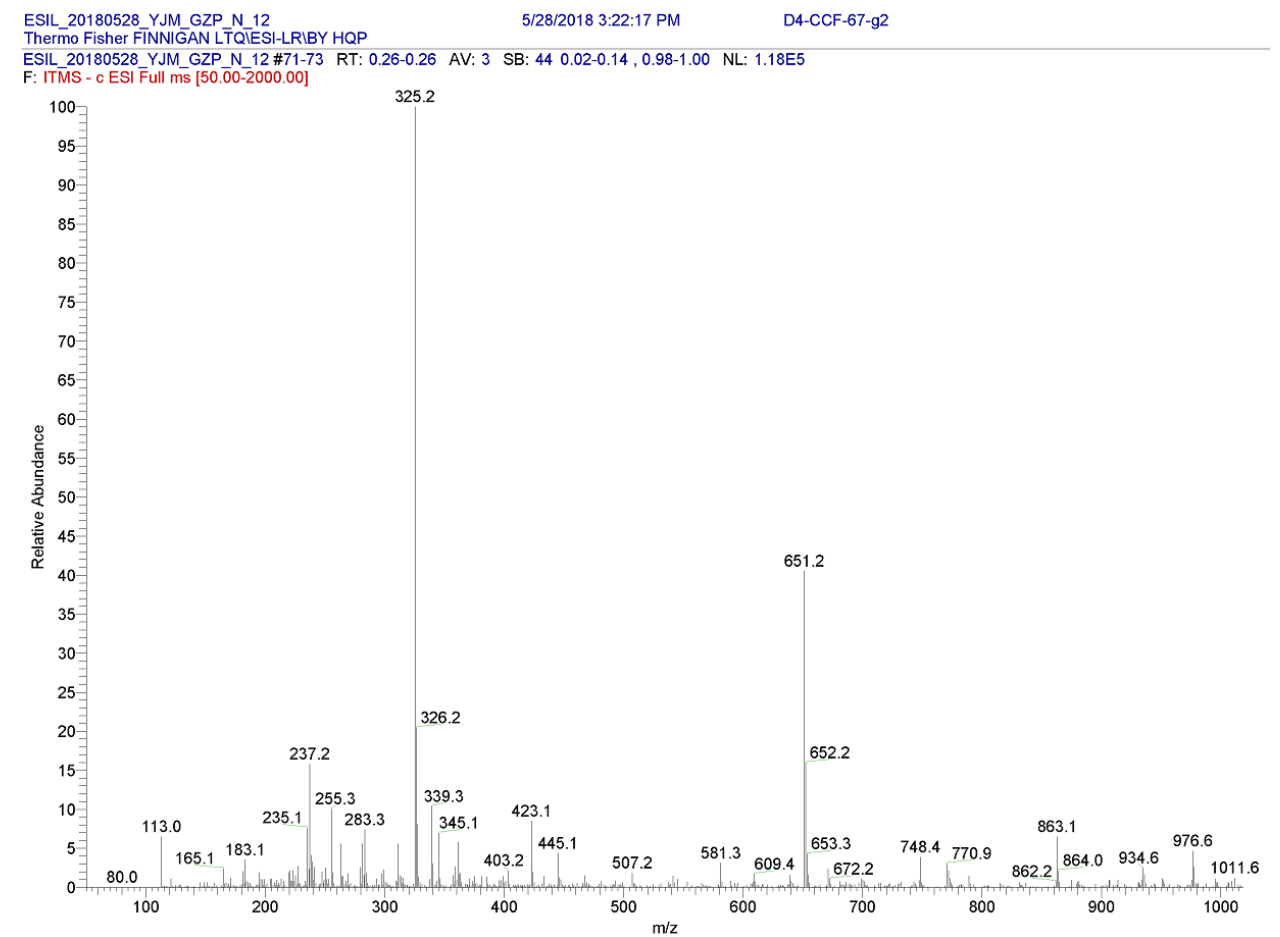


Figure S108. (+)-HRESIMS spectrum of fortalpinoid I (9).

Qualitative Analysis Report

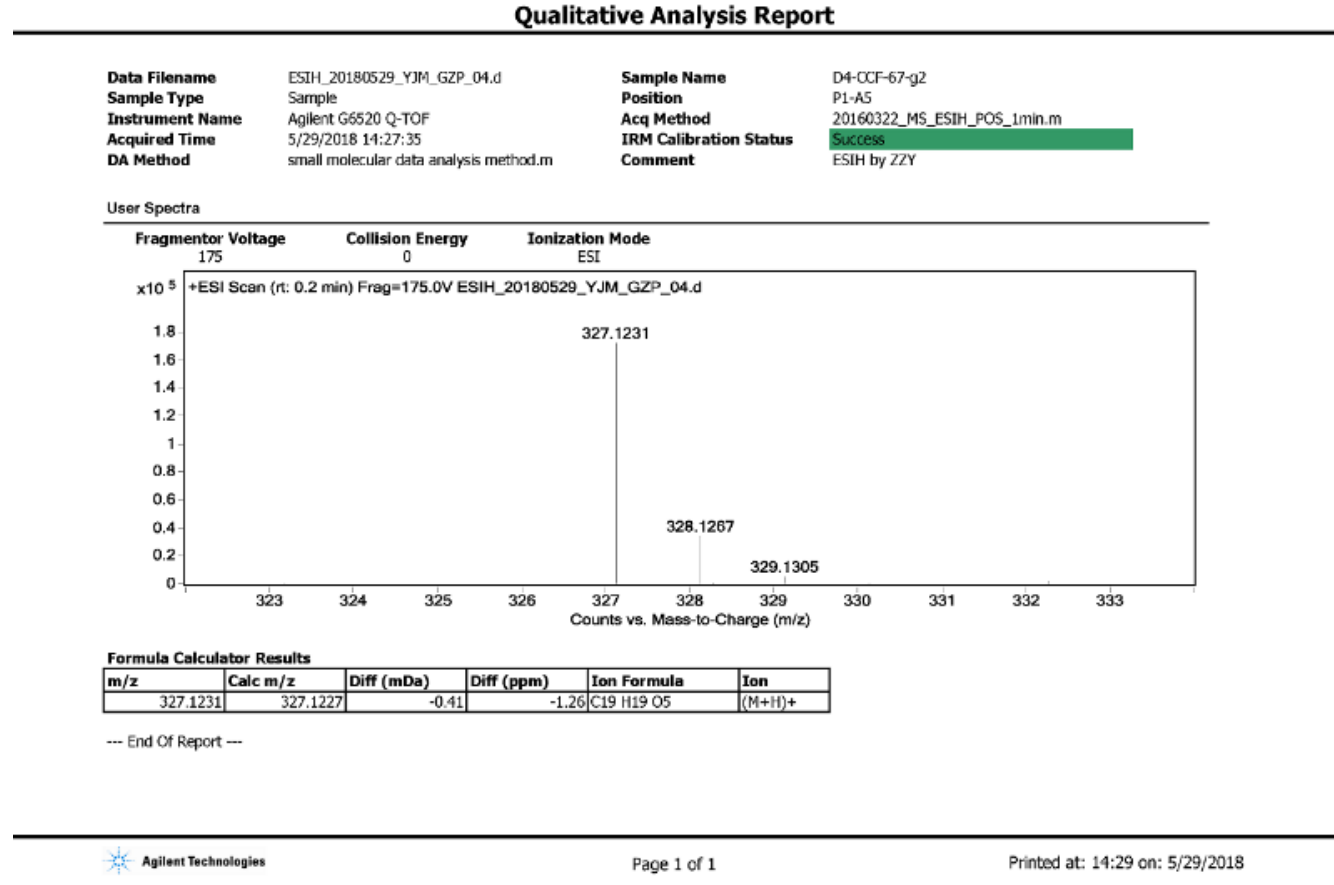

Figure S109. IR spectrum of fortalpinoid I (9).

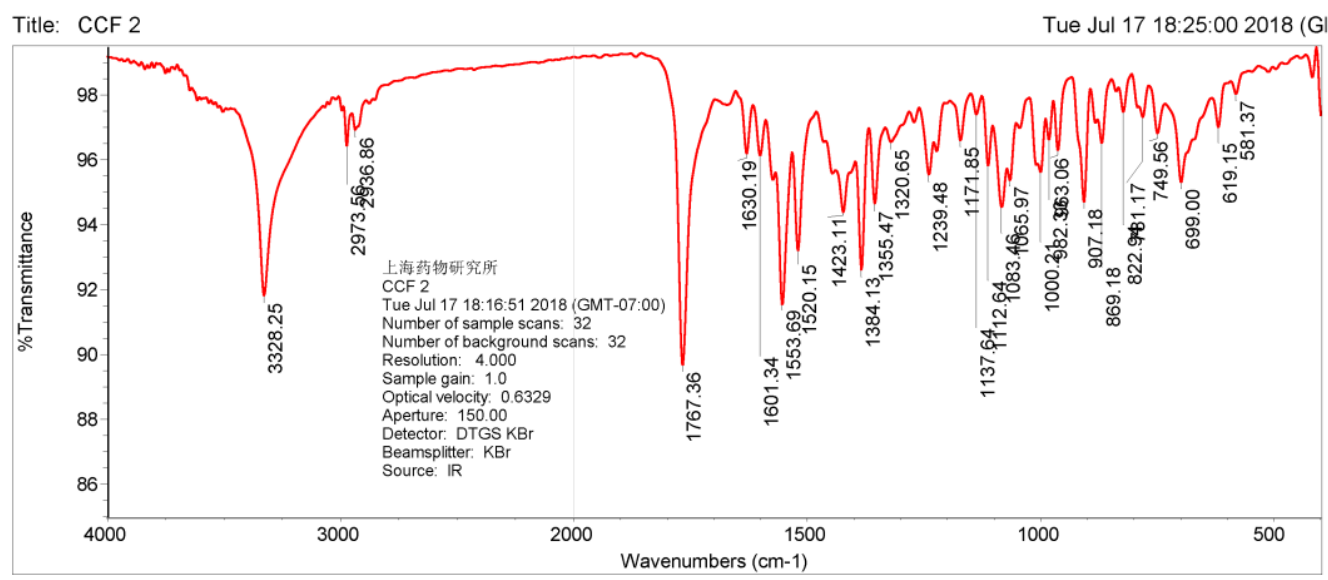

Collection time: Tue Jul 17 18:16:51 2018 (GMT-07 No search results for the selected spectrum!
No peak table for the selected spectrum! 
Figure S110. ${ }^{1} \mathrm{H}$ NMR spectrum of fortalpinoid J (10) in pyridine- $d 5$.

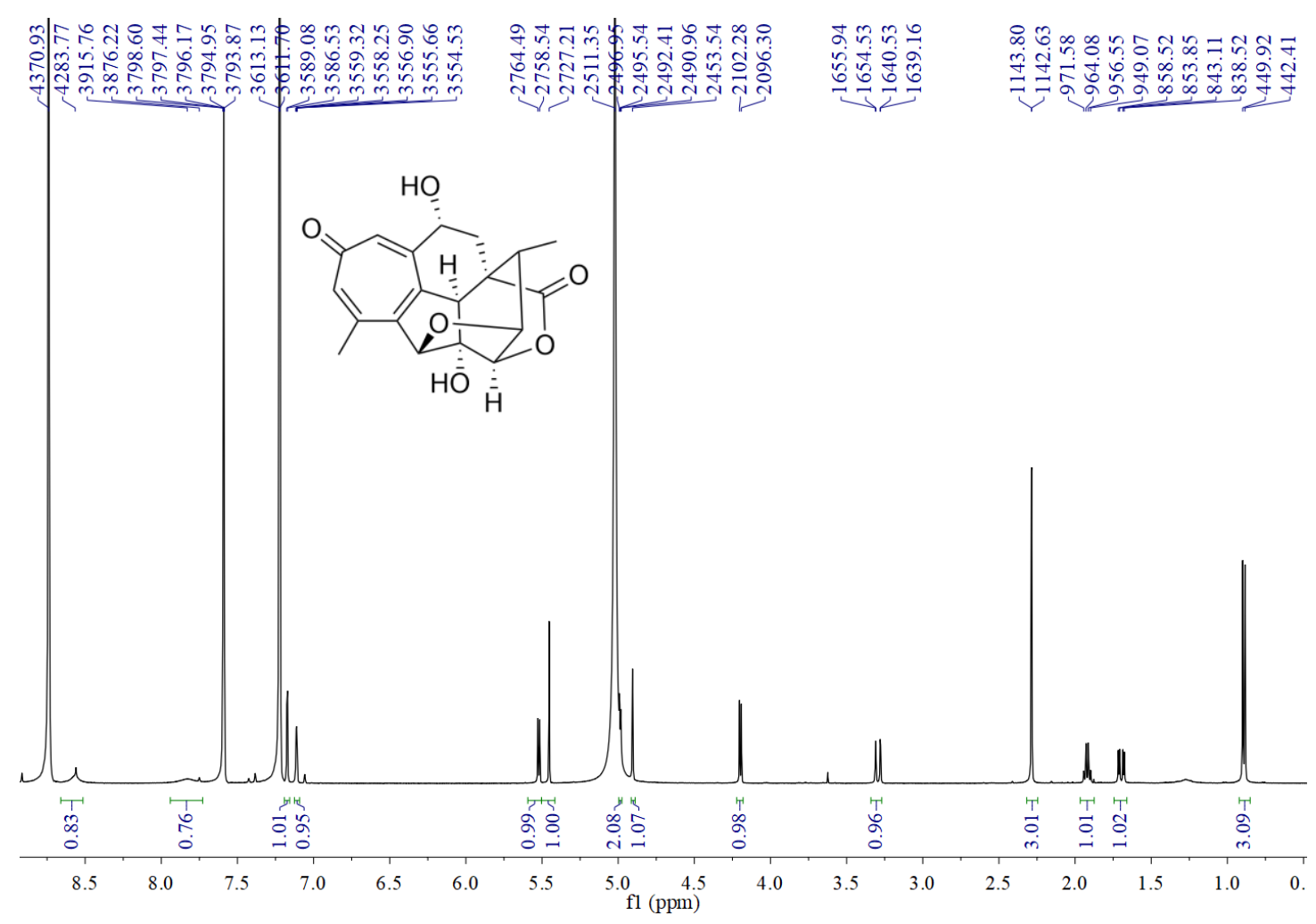

Figure S111. ${ }^{13} \mathrm{C}$ NMR spectrum of fortalpinoid $\mathrm{J}(\mathbf{1 0})$ in pyridine- $d 5$.

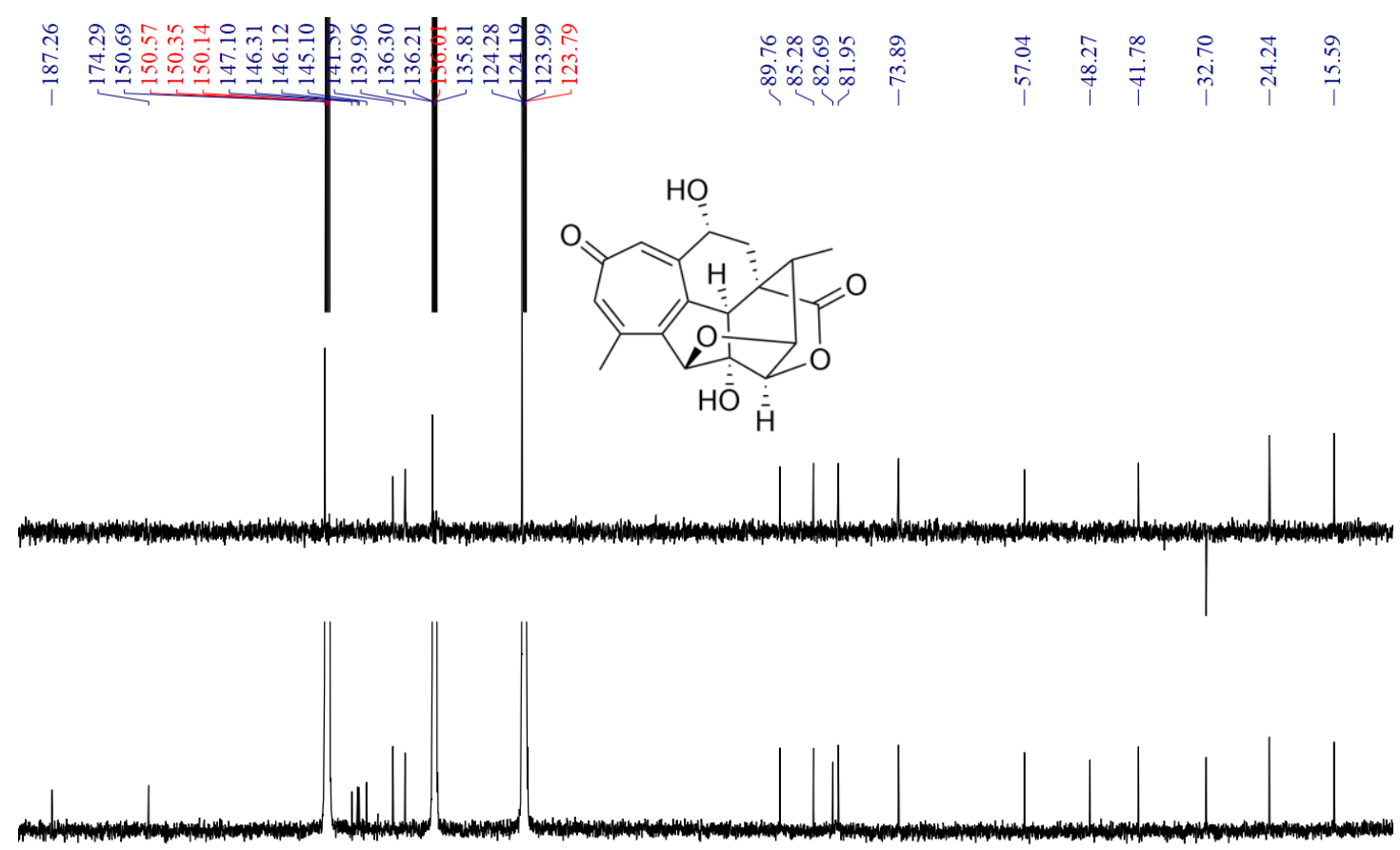

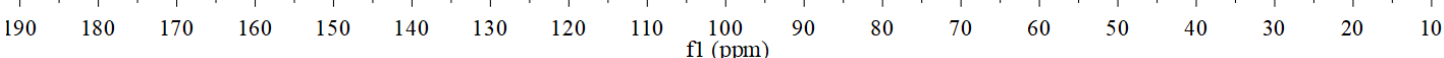


Figure S112. HSQC spectrum of fortalpinoid J (10) in pyridine- $d_{5}$.

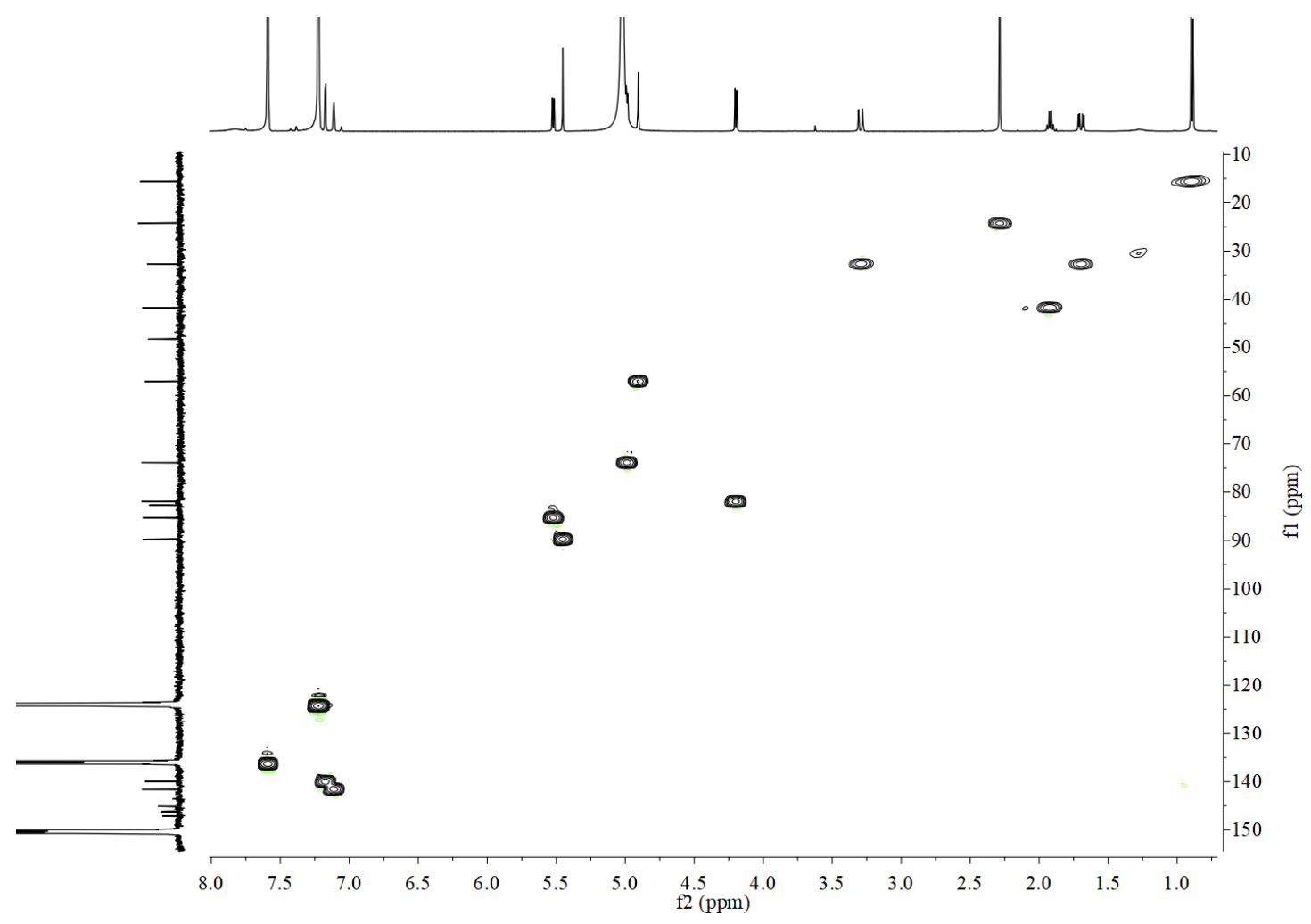

Figure S113. HMBC spectrum of fortalpinoid J (10) in pyridine- $d_{5}$.

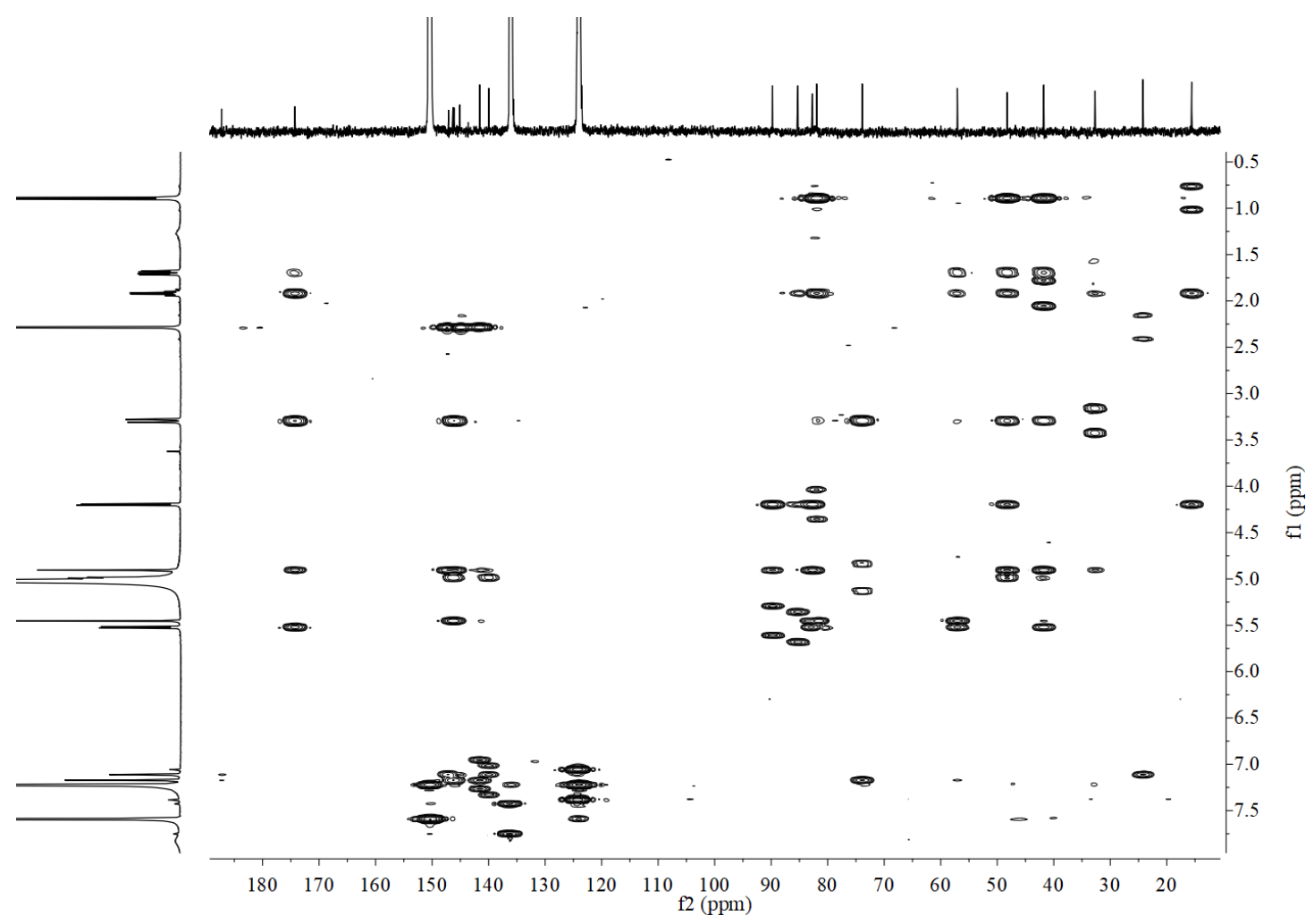


Figure S114. ${ }^{1} \mathrm{H}-{ }^{1} \mathrm{H}$ COSY spectrum of fortalpinoid J (10) in pyridine- $d 5$.

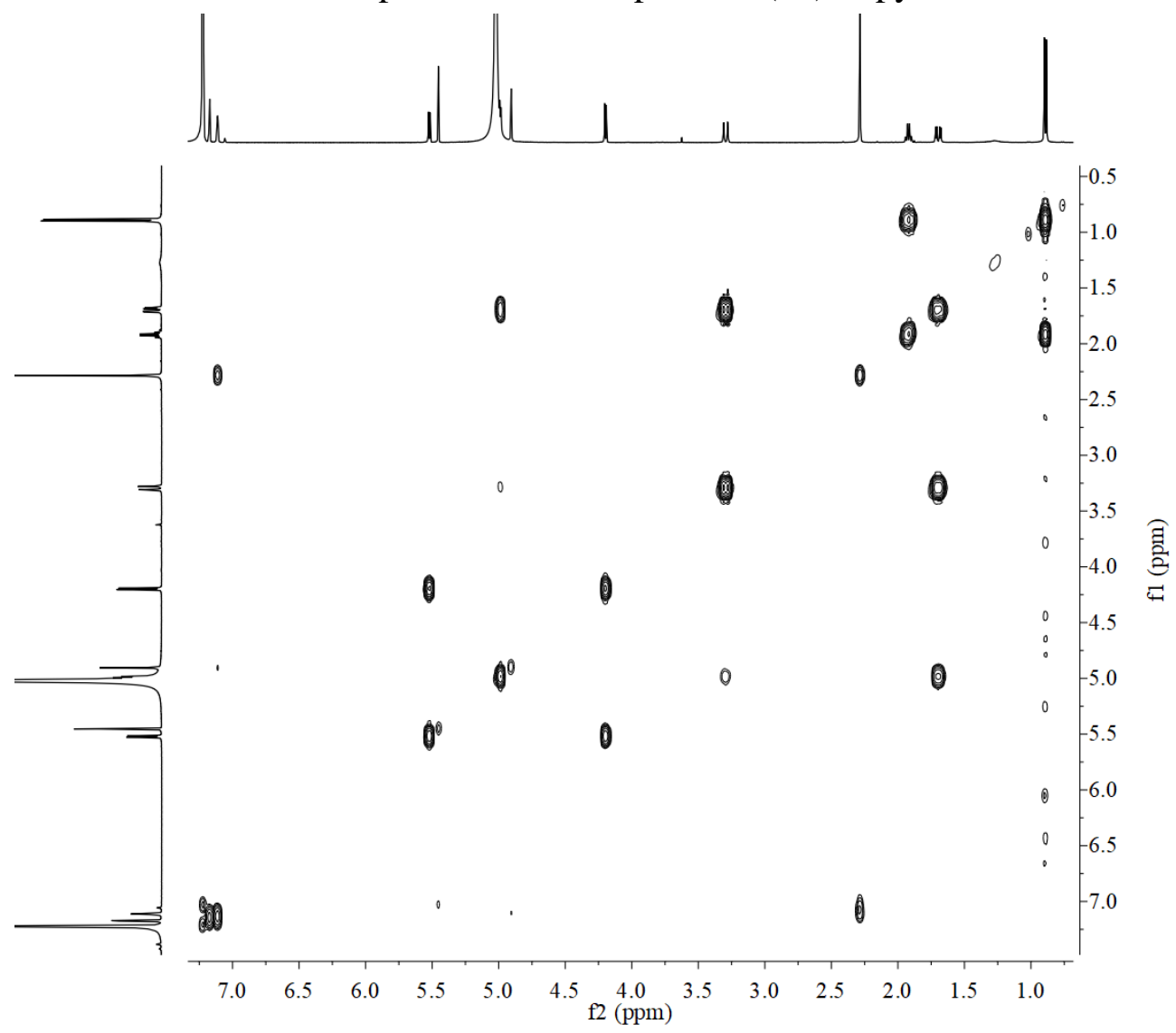

Figure S115. NOESY spectrum of fortalpinoid J (10) in pyridine- $d_{5}$.

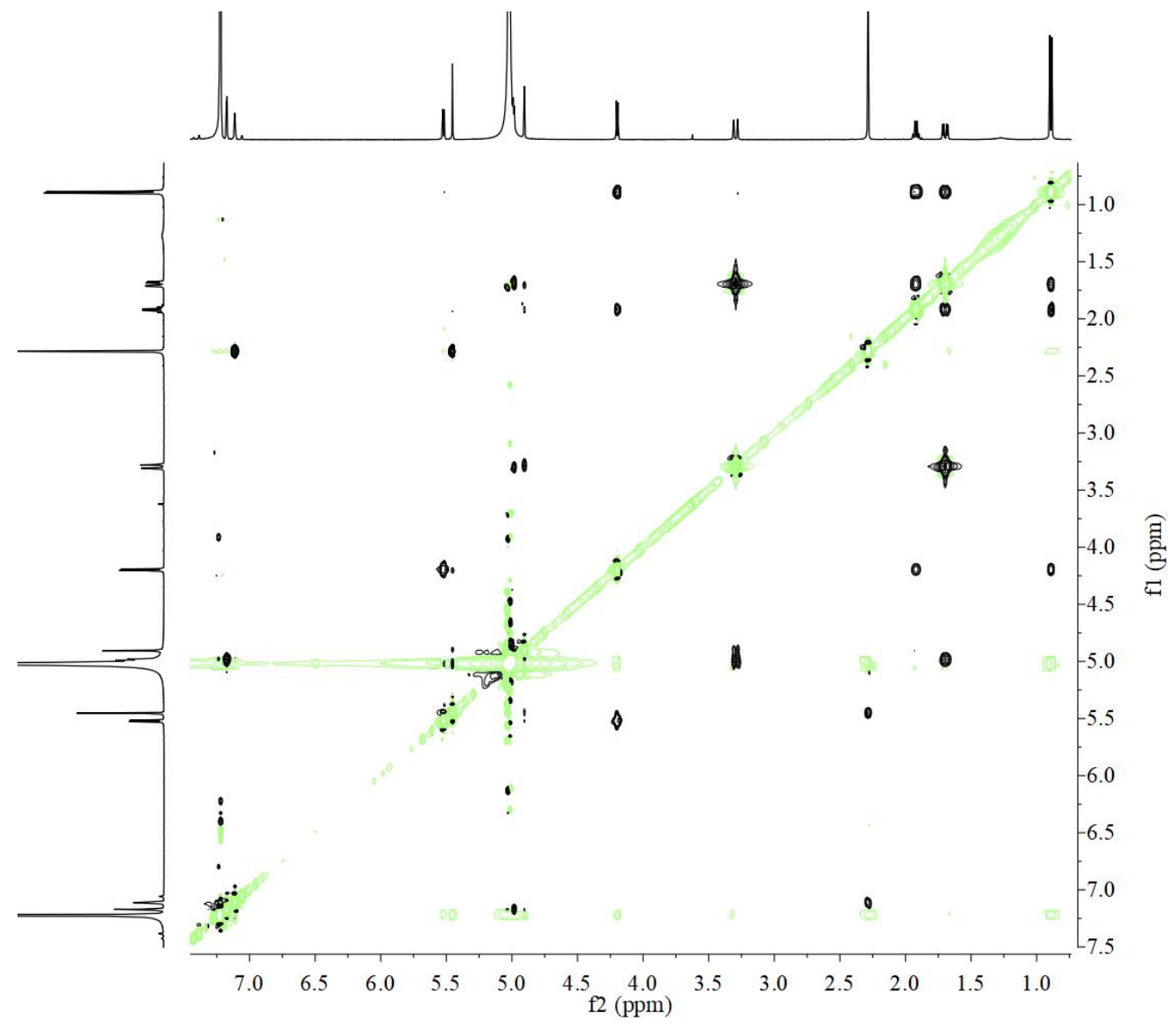


Figure S116. (+)-ESIMS spectrum of fortalpinoid J (10).

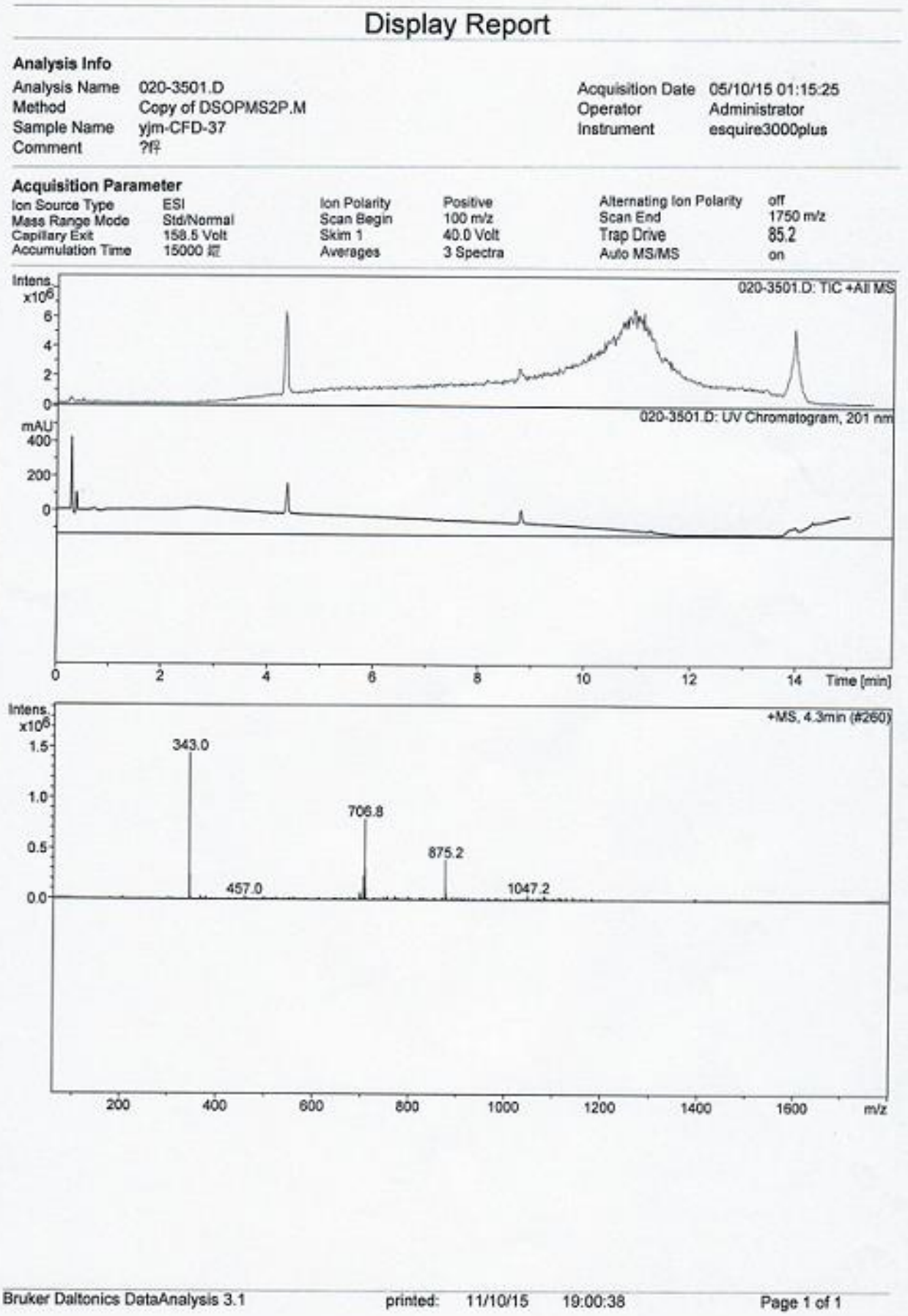


Figure S117. (-)-ESIMS spectrum of fortalpinoid J (10).

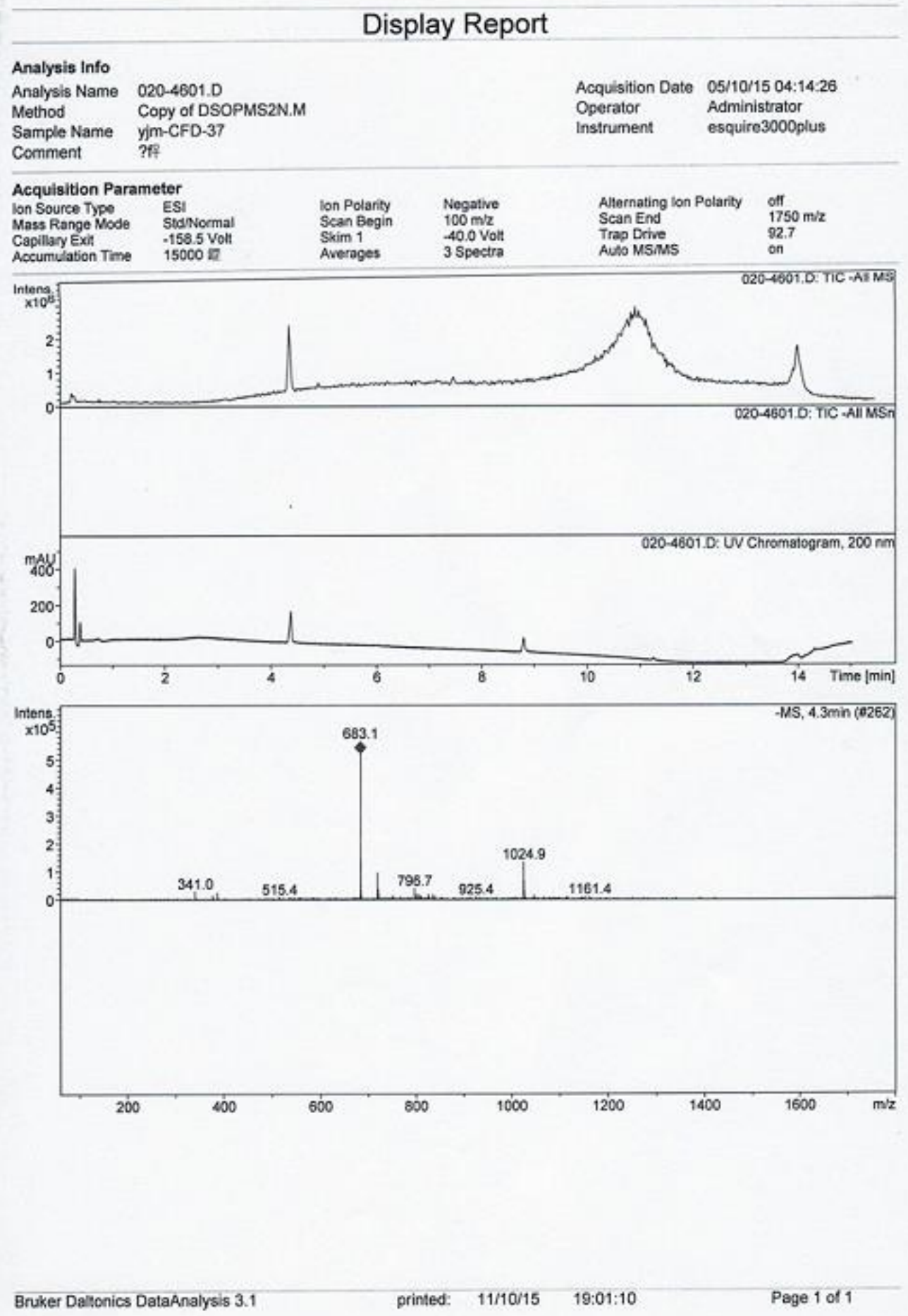


Figure S118. (-)-HRESIMS spectrum of fortalpinoid J (10).

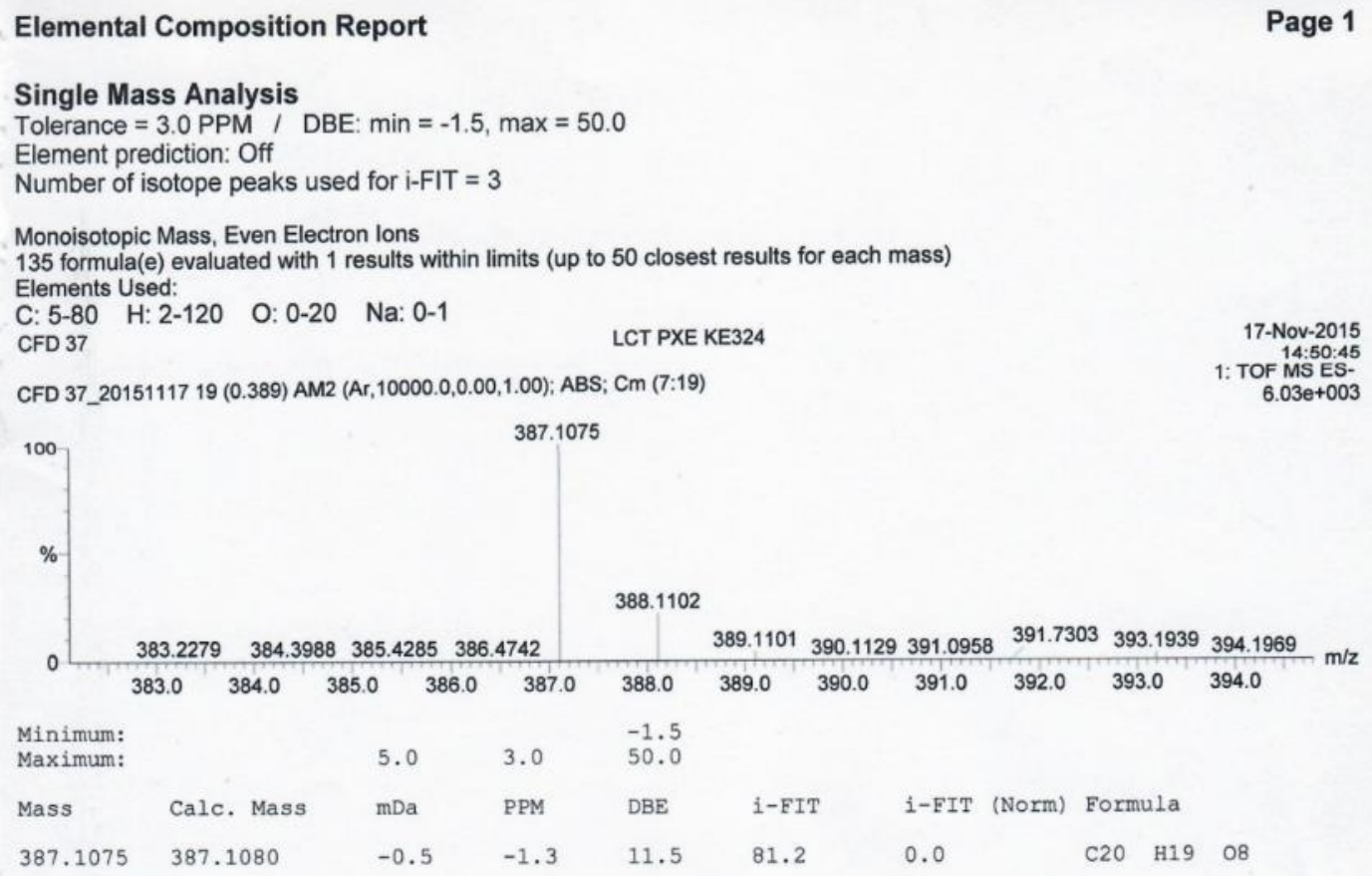

Figure S119. IR spectrum of fortalpinoid J (10).

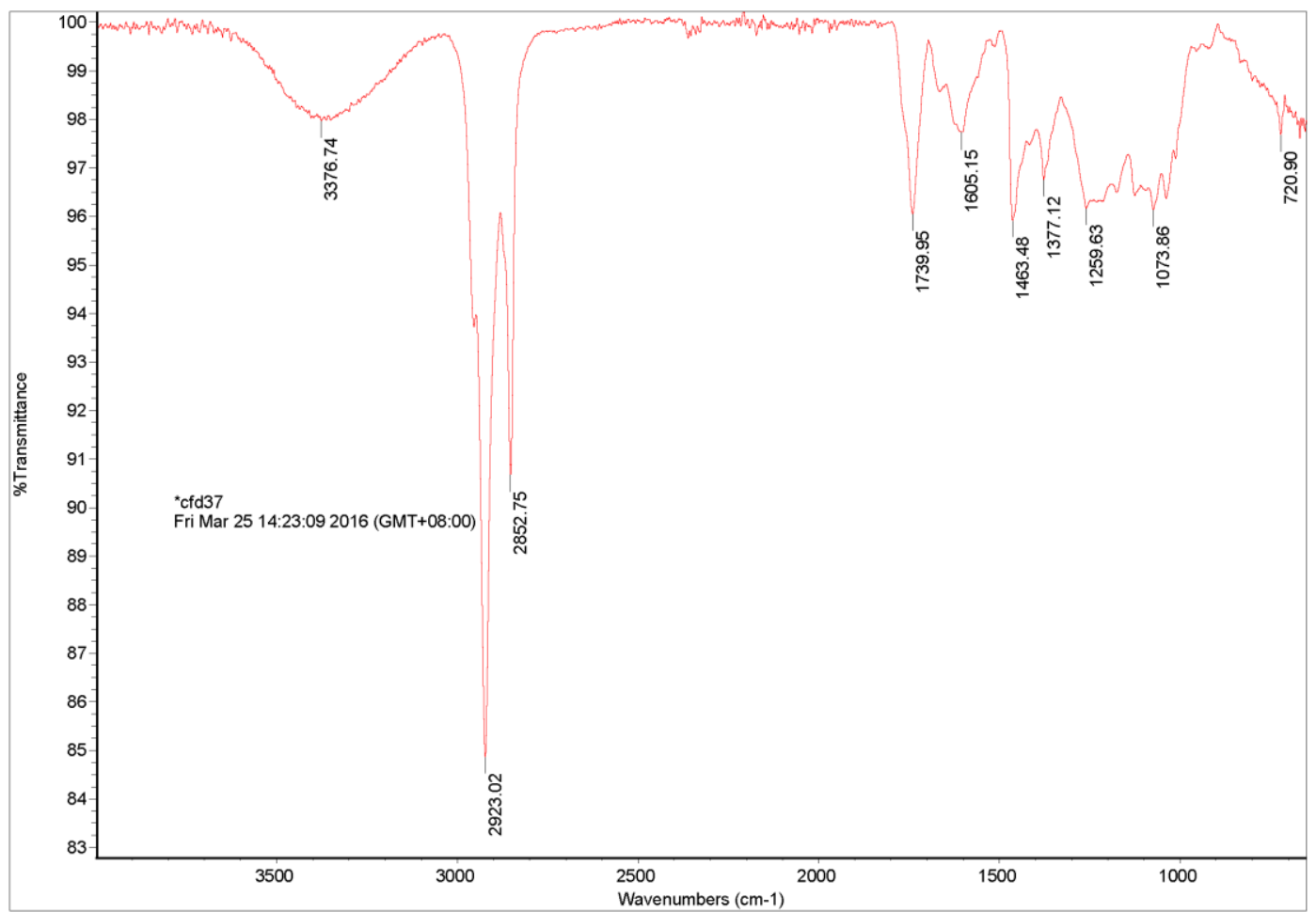


Figure S120. ${ }^{1} \mathrm{H}$ NMR spectrum of fortalpinoid $\mathrm{K}(\mathbf{1 1})$ in $\mathrm{CDCl}_{3}$.

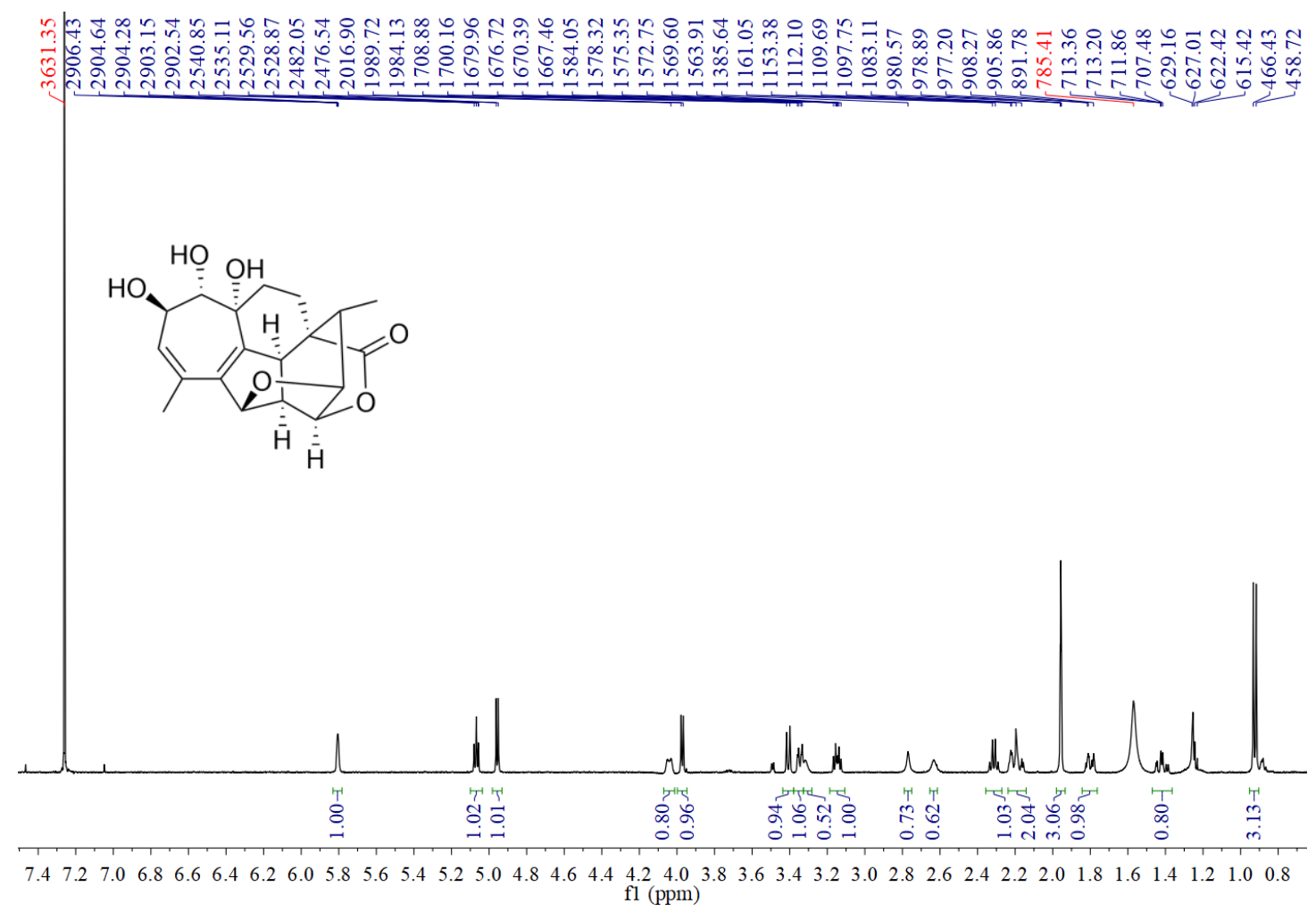

Figure S121. ${ }^{13} \mathrm{C}$ NMR spectrum of fortalpinoid $\mathrm{K}(11)$ in $\mathrm{CDCl}_{3}$.
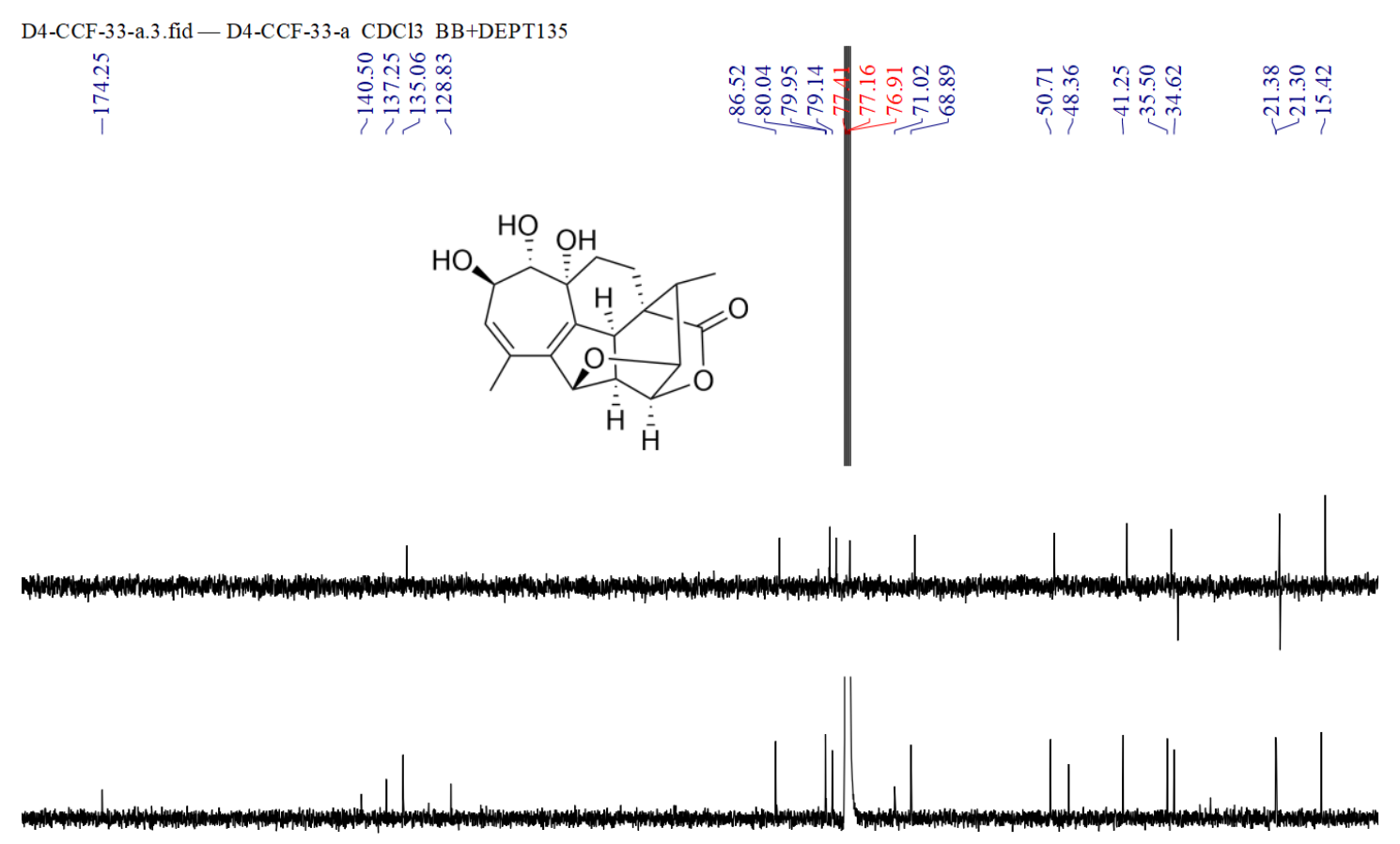

180

$170 \quad 160$

$150 \quad 140$

$120 \quad 110$

$\begin{array}{ll}100 & 90 \\ \mathrm{fl}(\mathrm{ppm}) & 80\end{array}$

$70 \quad 60$

50

40

$30-20 \quad 10$ 
Figure S122. HSQC spectrum of fortalpinoid $\mathrm{K}(\mathbf{1 1})$ in $\mathrm{CDCl}_{3}$.

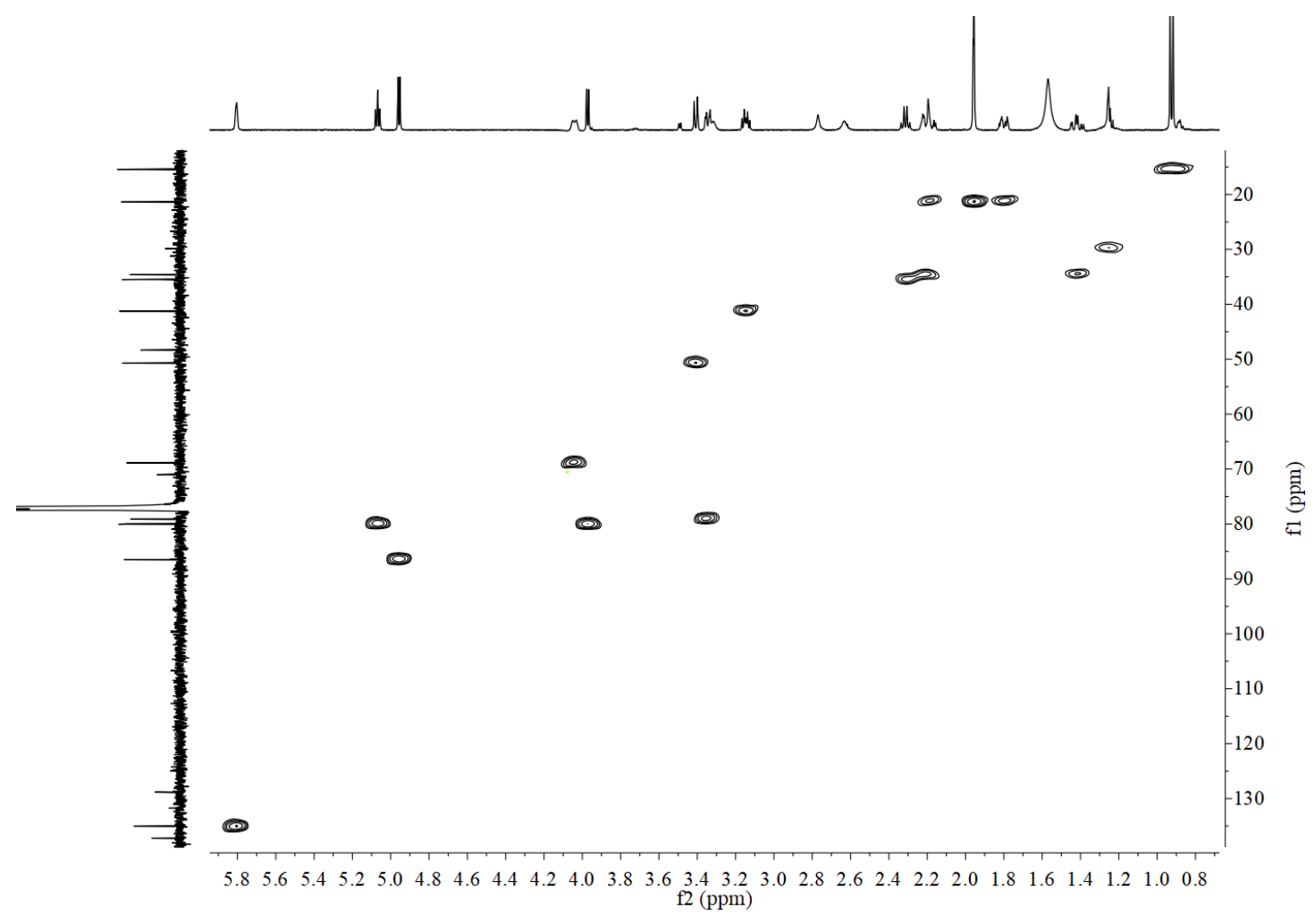

Figure S123. HMBC spectrum of fortalpinoid $\mathrm{K}(\mathbf{1 1})$ in $\mathrm{CDCl}_{3}$.

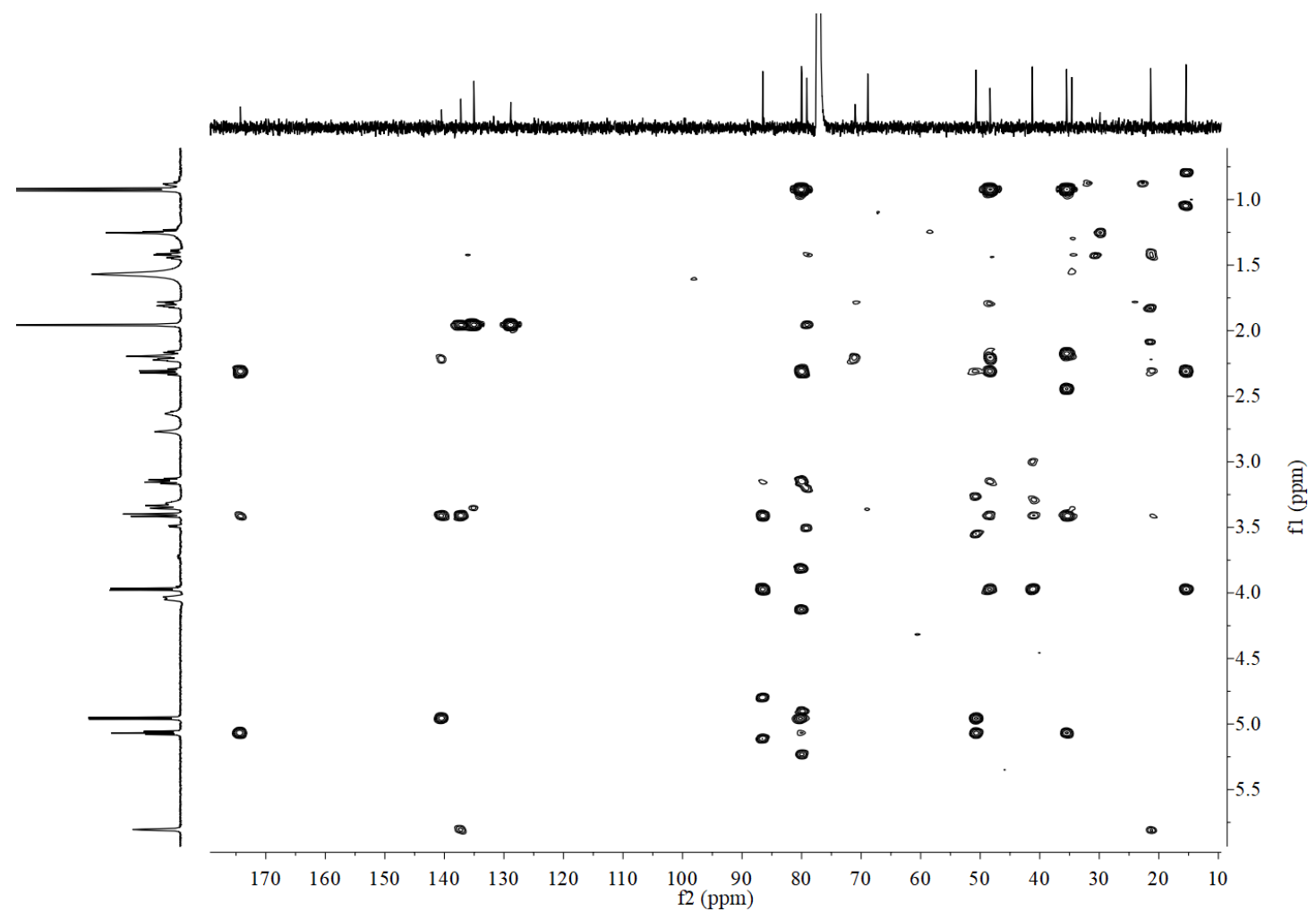


Figure S124. ${ }^{1} \mathrm{H}-{ }^{1} \mathrm{H}$ COSY spectrum of fortalpinoid $\mathrm{K}(\mathbf{1 1})$ in $\mathrm{CDCl}_{3}$.

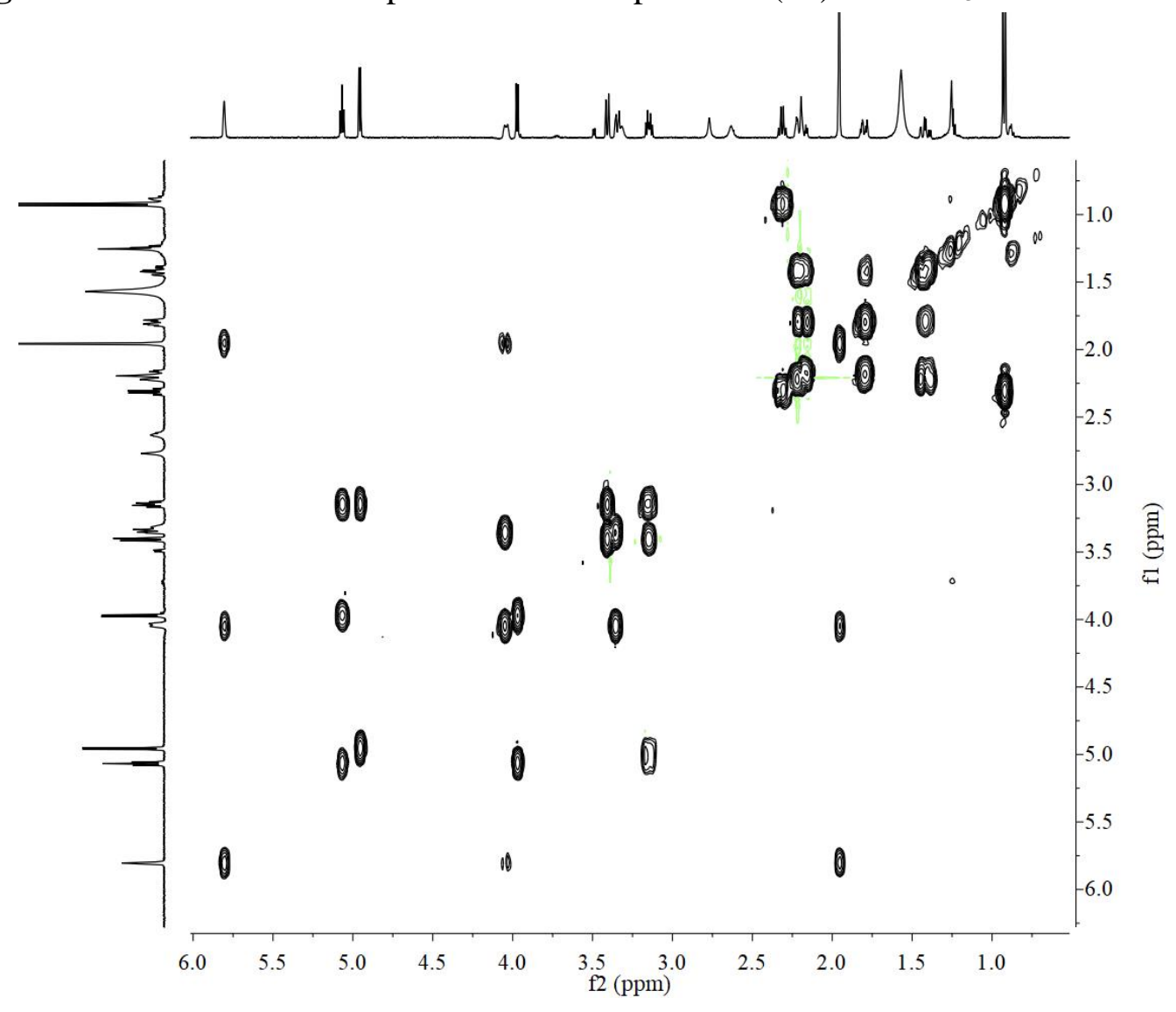

Figure S125. ROESY spectrum of fortalpinoid $\mathrm{K}(\mathbf{1 1})$ in $\mathrm{CDCl}_{3}$.

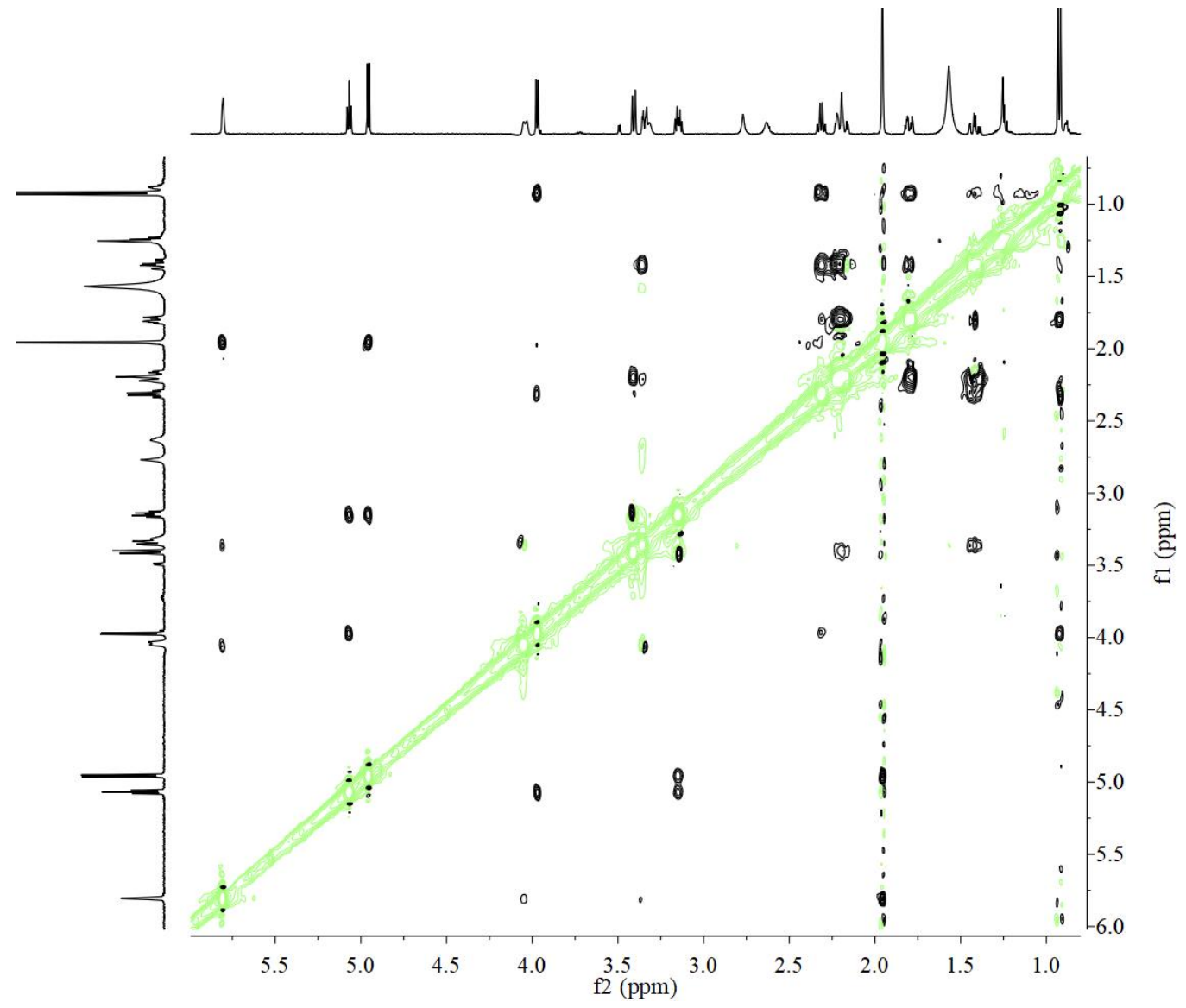


Figure S126. ${ }^{1} \mathrm{H}$ NMR spectrum of fortalpinoid K (11) in pyridine- $d 5$.

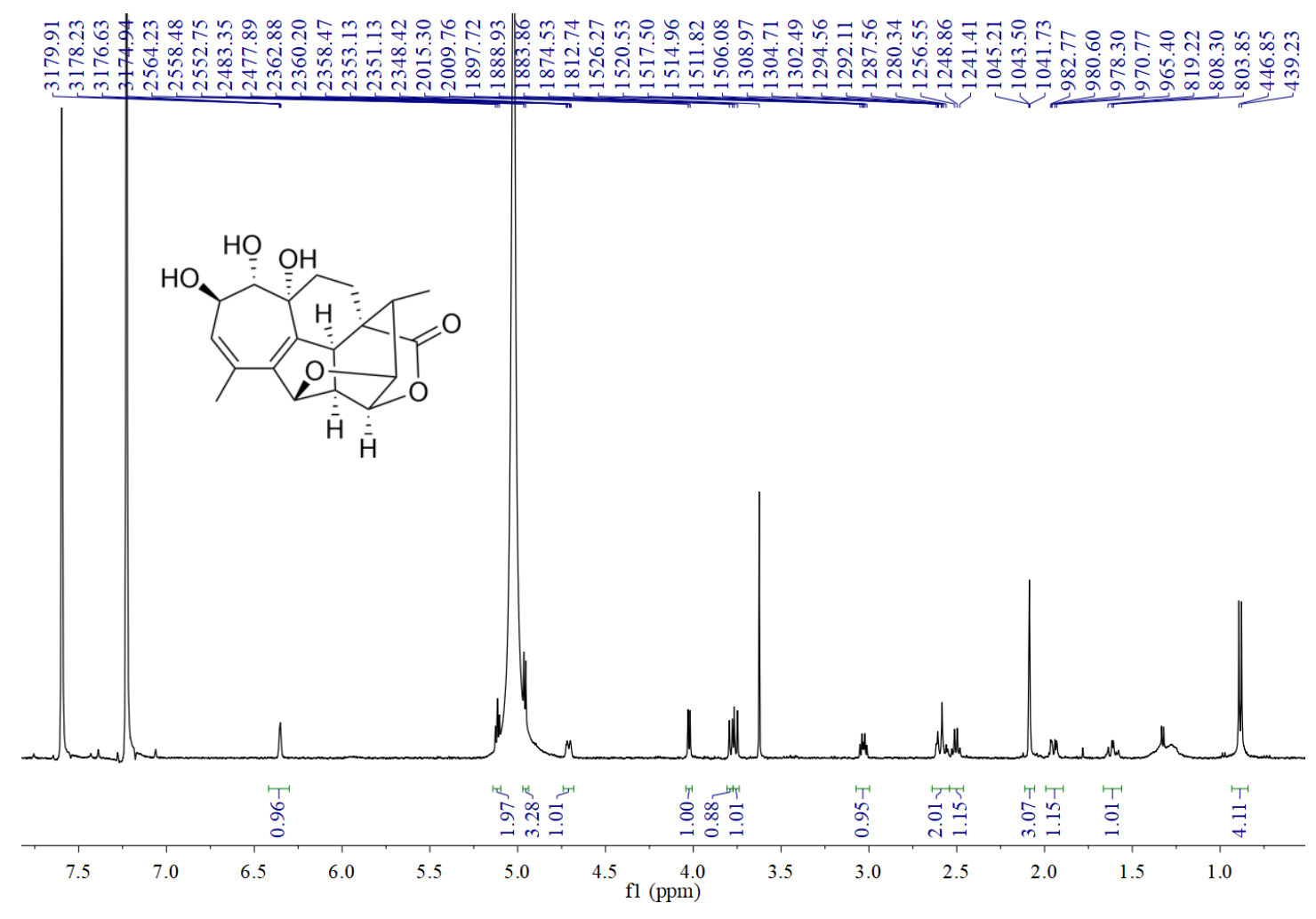

Figure S127. ${ }^{13} \mathrm{C}$ NMR spectrum of fortalpinoid K (11) in pyridine- $d 5$.
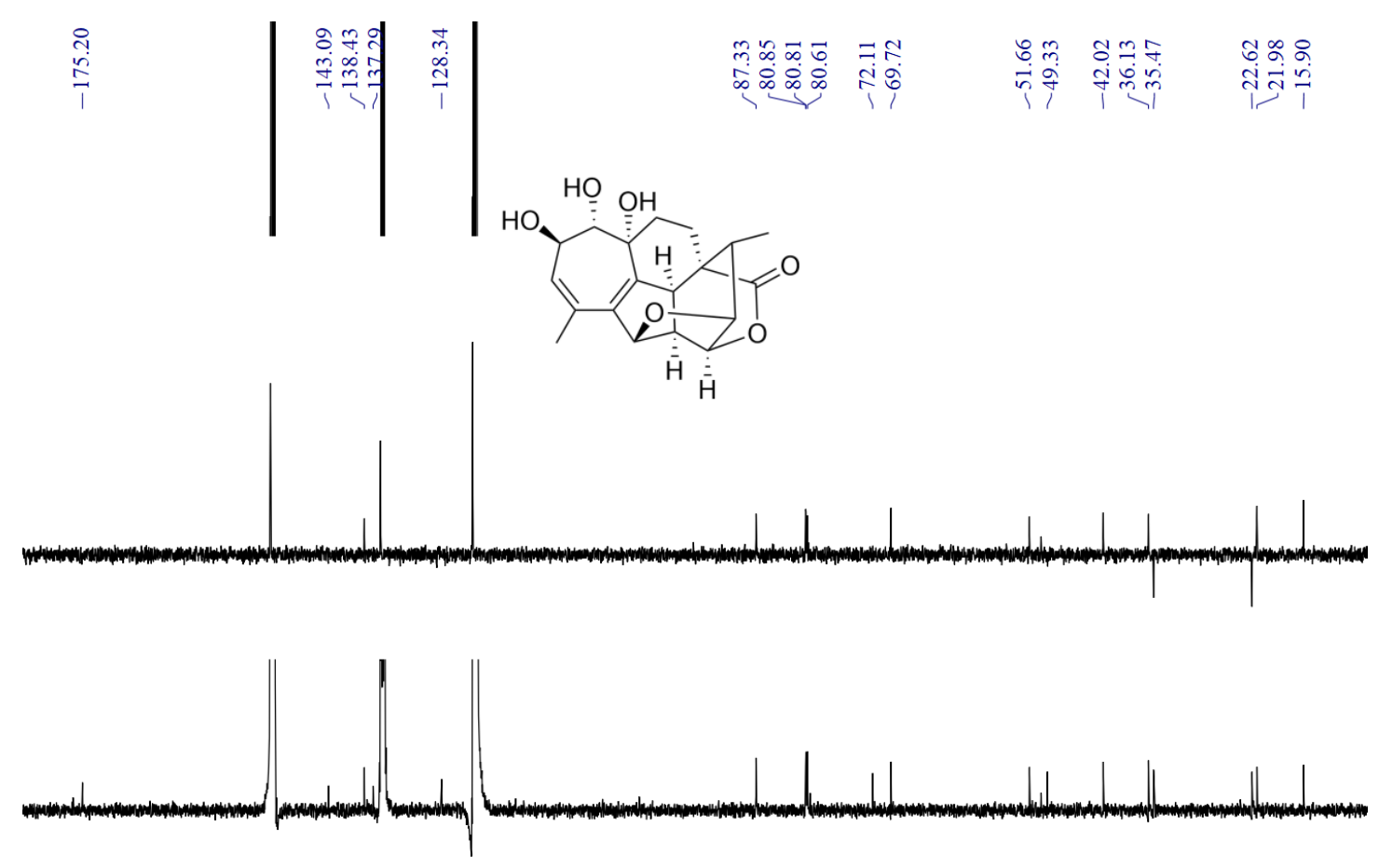

$\begin{array}{rrrrrrrrr}180 & 170 & 160 & 150 & 140 & 130 & 120 & 110 & \begin{array}{c}100 \\ \text { fl (ppm) }\end{array}\end{array}$ 
Figure S128. HSQC spectrum of fortalpinoid K (11) in pyridine- $d_{5}$.

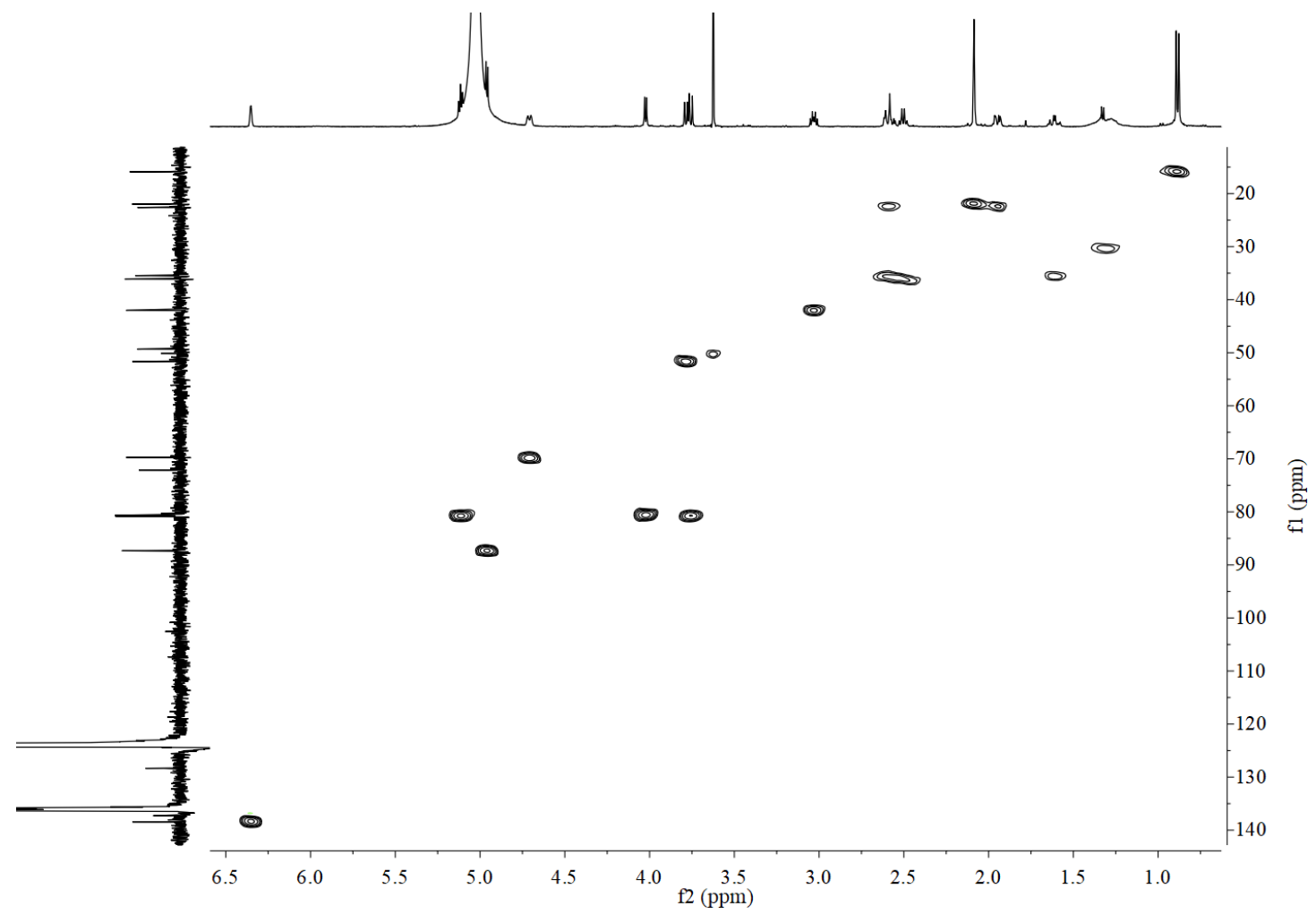

Figure S129. HMBC spectrum of fortalpinoid K (11) in pyridine- $d 5$.

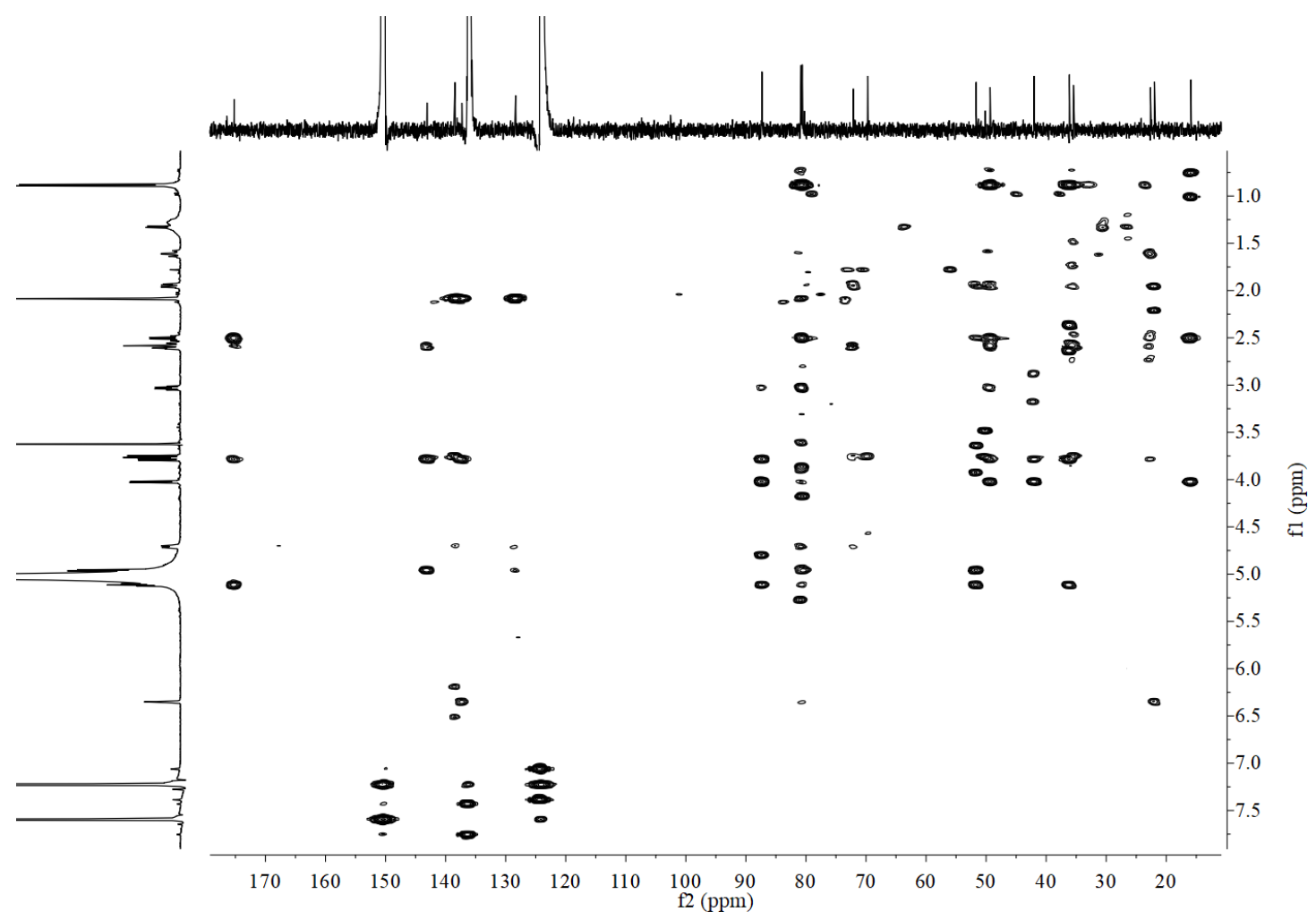


Figure S130. ${ }^{1} \mathrm{H}-{ }^{1} \mathrm{H}$ COSY spectrum of fortalpinoid $\mathrm{K}(\mathbf{1 1})$ in pyridine- $d 5$.

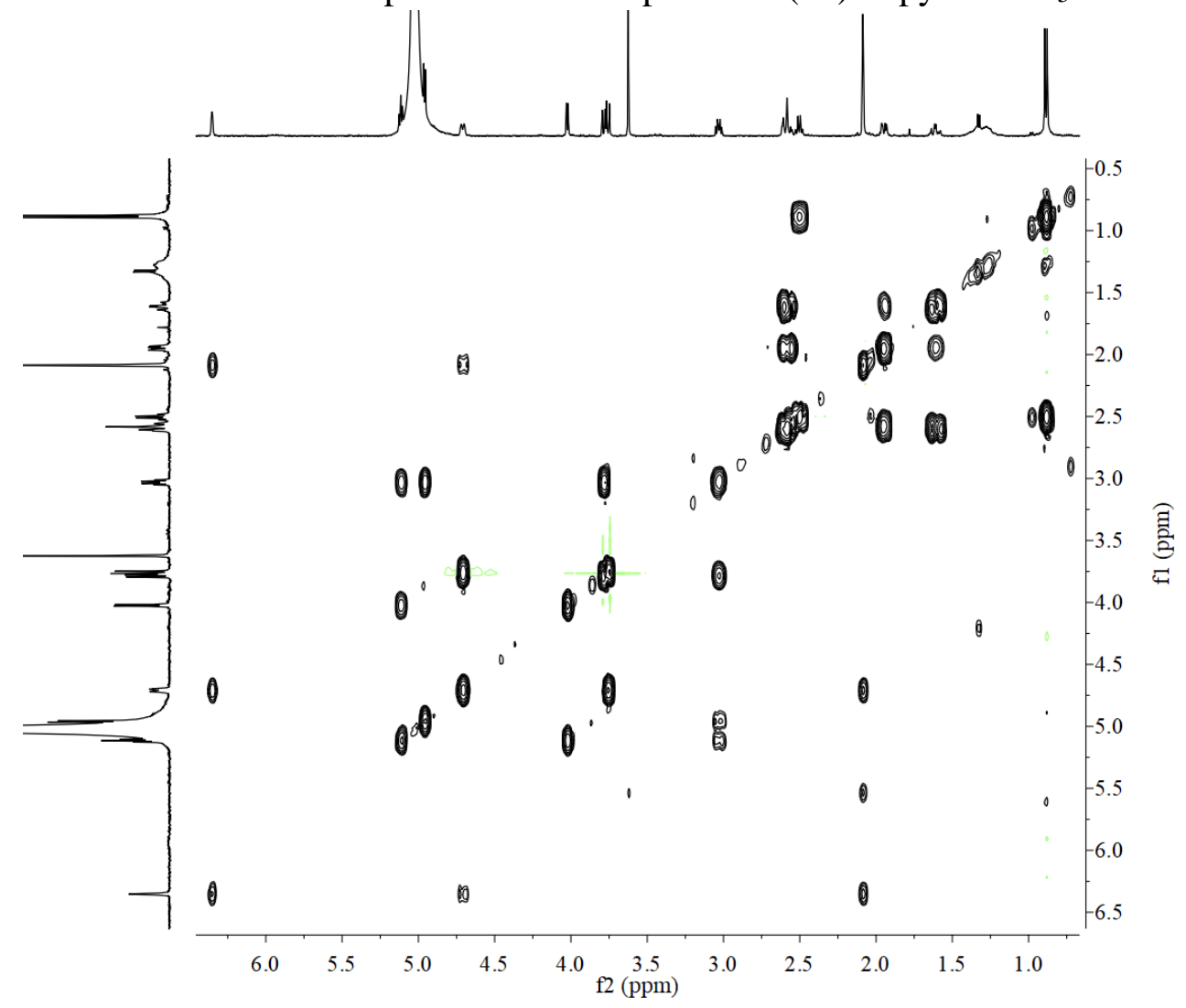

Figure S131. NOESY spectrum of fortalpinoid K (11) in pyridine- $d_{5}$.

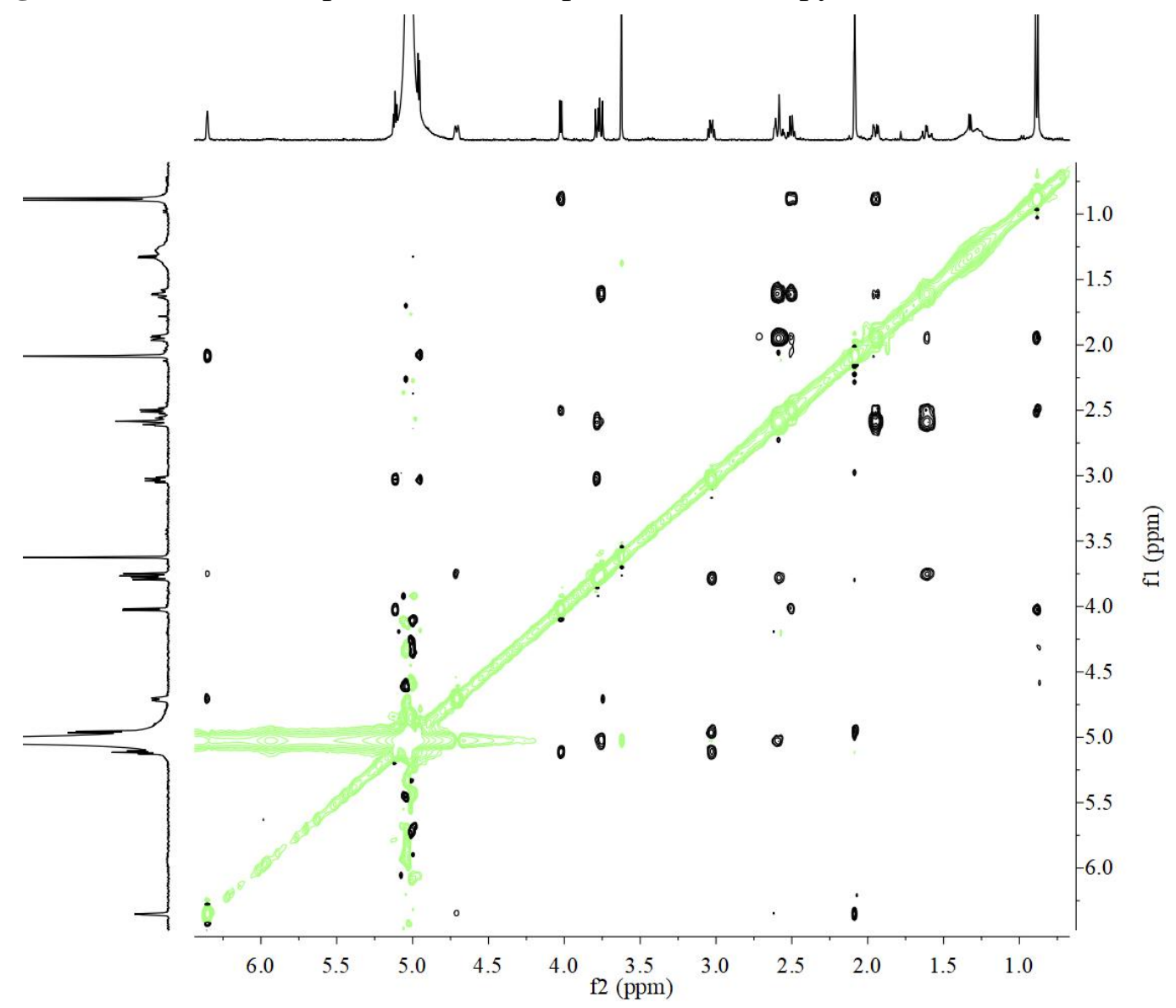


Figure S132. (+)-ESIMS spectrum of fortalpinoid K (11).

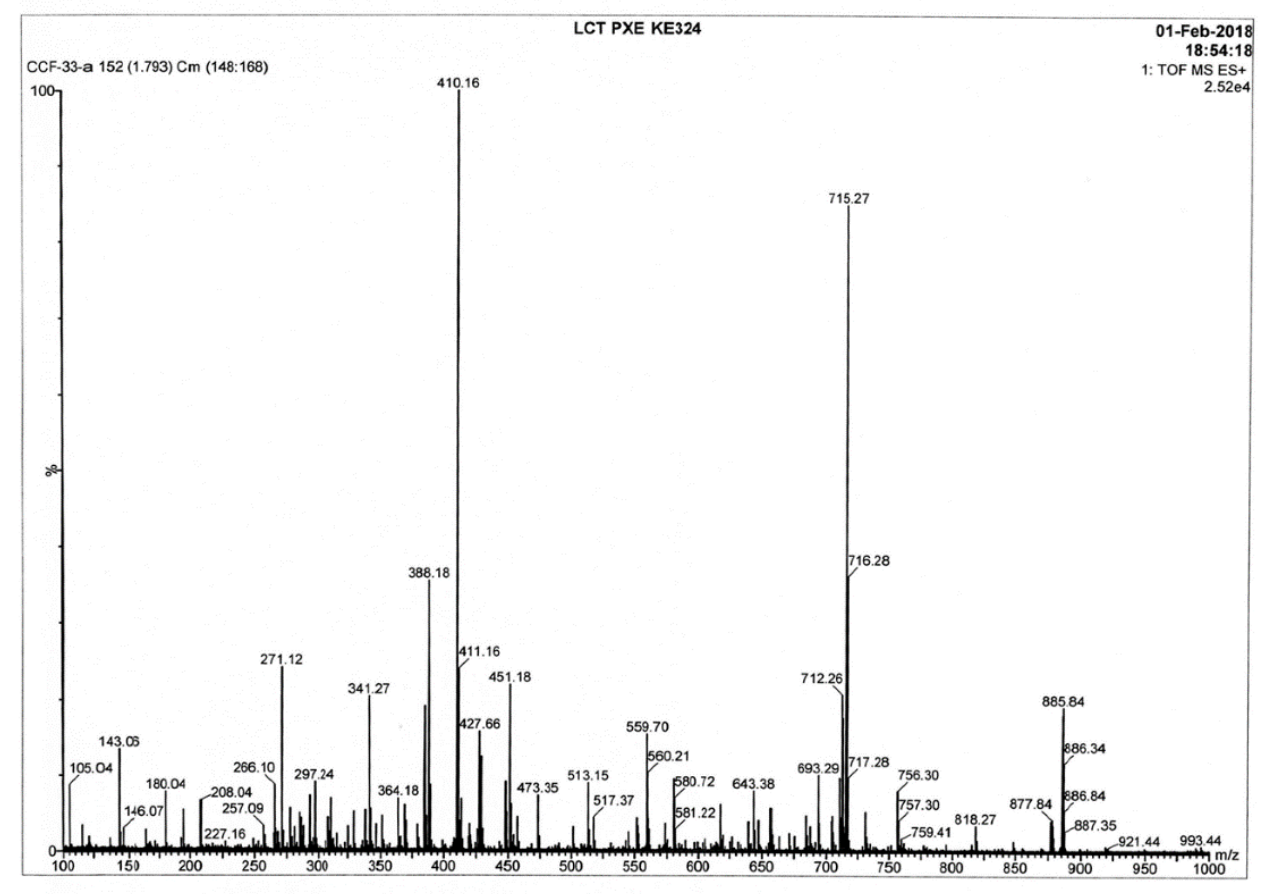

Figure S133. (-)-ESIMS spectrum of fortalpinoid K (11).

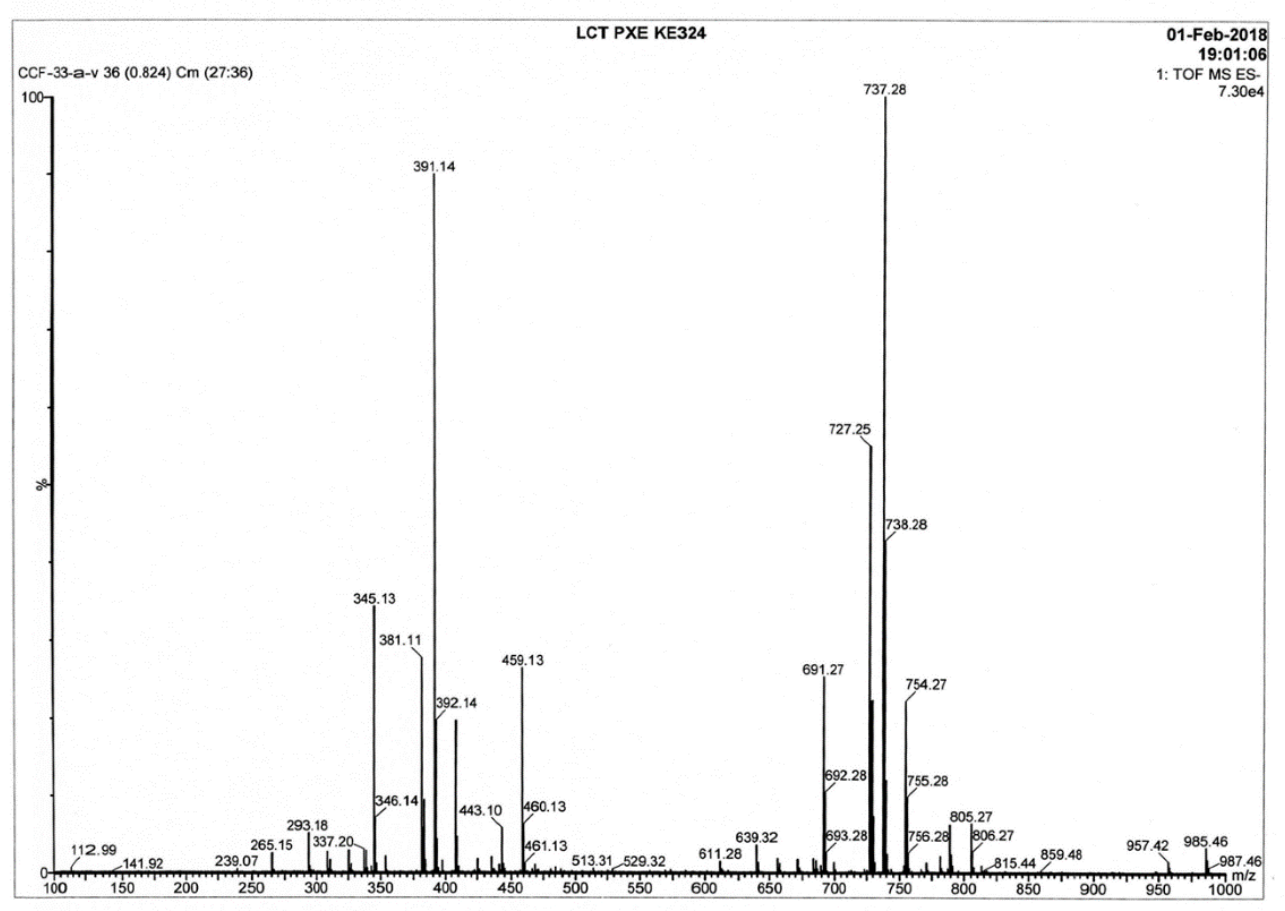


Figure S134. (-)-HRESIMS spectrum of fortalpinoid K (11).

Elemental Composition Report

Page 1

Single Mass Analysis

Tolerance $=3.0$ PPM / DBE: $\min =-1.5, \max =50.0$

Number of isotope peaks used for i-FIT $=3$

Monoisotopic Mass, Even Electron Ions

124 formula(e) evaluated with 1 results within limits (up to 50 best isotopic matches for each mass)

$\begin{array}{lll}\text { Clements Used: } 0-100 \text { H: } 0-1000 & 0: 0-500 \quad \mathrm{Na}: 0-1\end{array}$

LCT PXE KES

01-Feb-2018

$18: 58: 11$
CCF-33-a- 149 (1.742) $\mathrm{Cm}(136: 160)$

345.1339

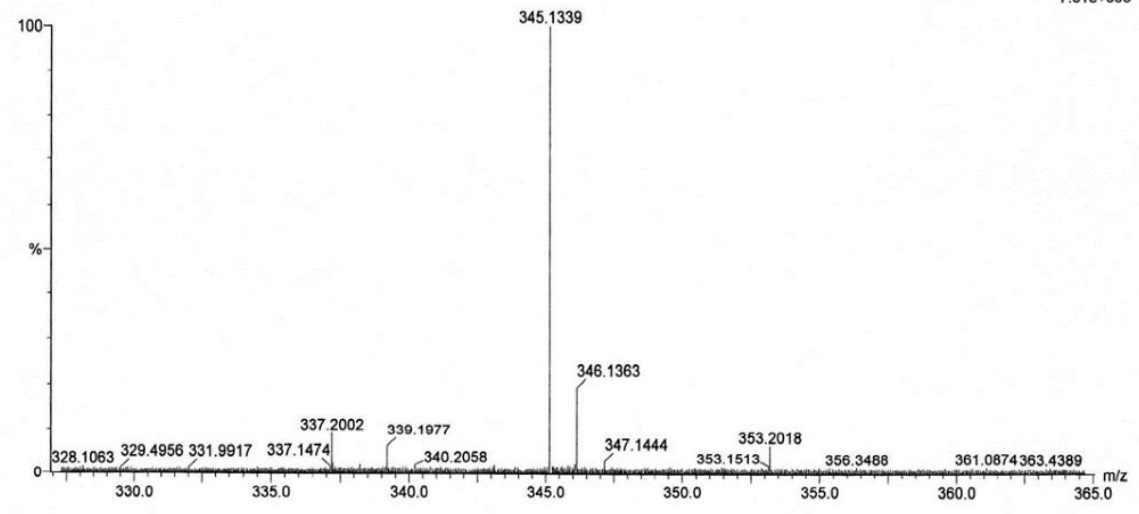

$\begin{array}{llll}\text { Minimum: } & & & -1.5 \\ \text { Maximum: } & 5.0 & 3.0 & 50.0\end{array}$

Mass Calc. Mass mDa PPM DBE

$\begin{array}{llllll}9.5 & 427.5 & 0.0 & \text { C19 } & \text { H21 } & 06\end{array}$

Figure S135. IR spectrum of fortalpinoid K (11).

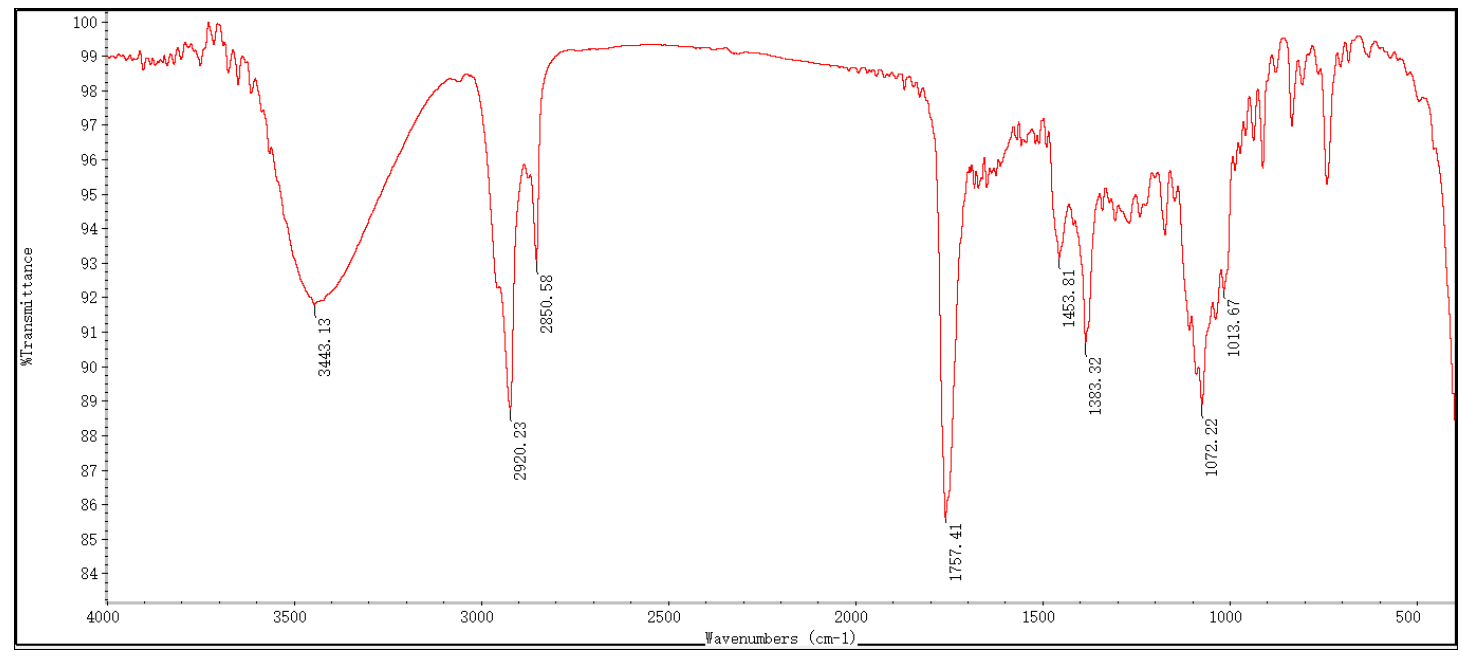


Figure S136. ECD spectra of fortalpinoid K (11).

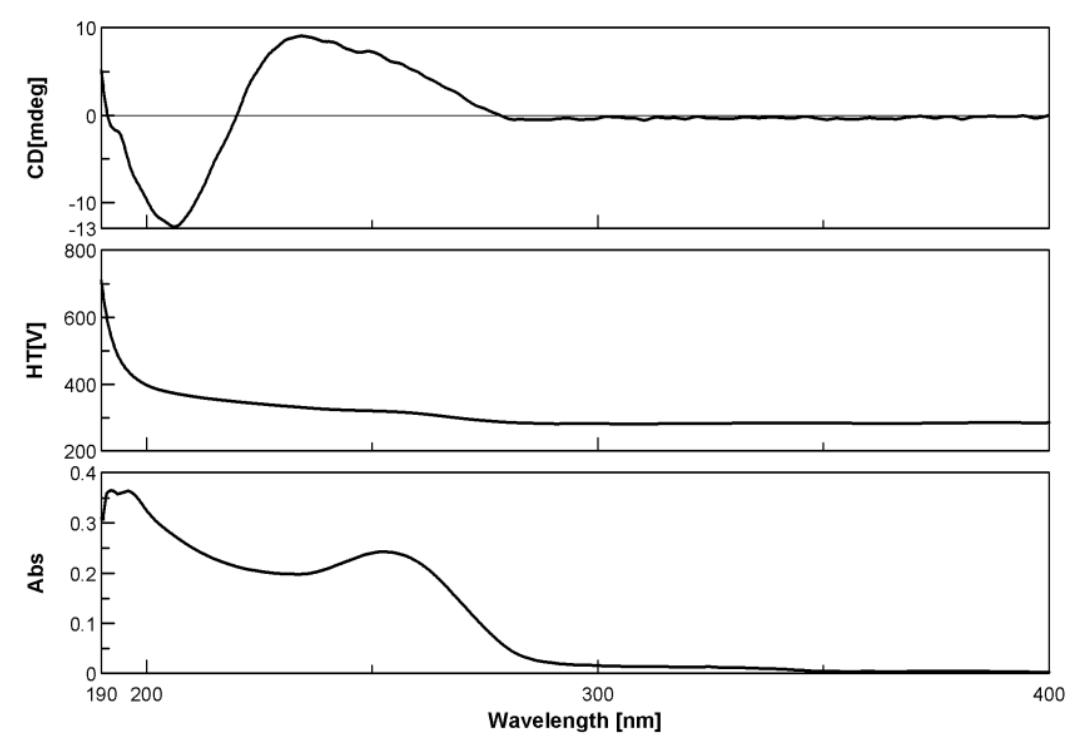

Figure S137. ${ }^{1} \mathrm{H}$ NMR spectrum of fortalpinoid L (12) in methanol- $d_{4}$.

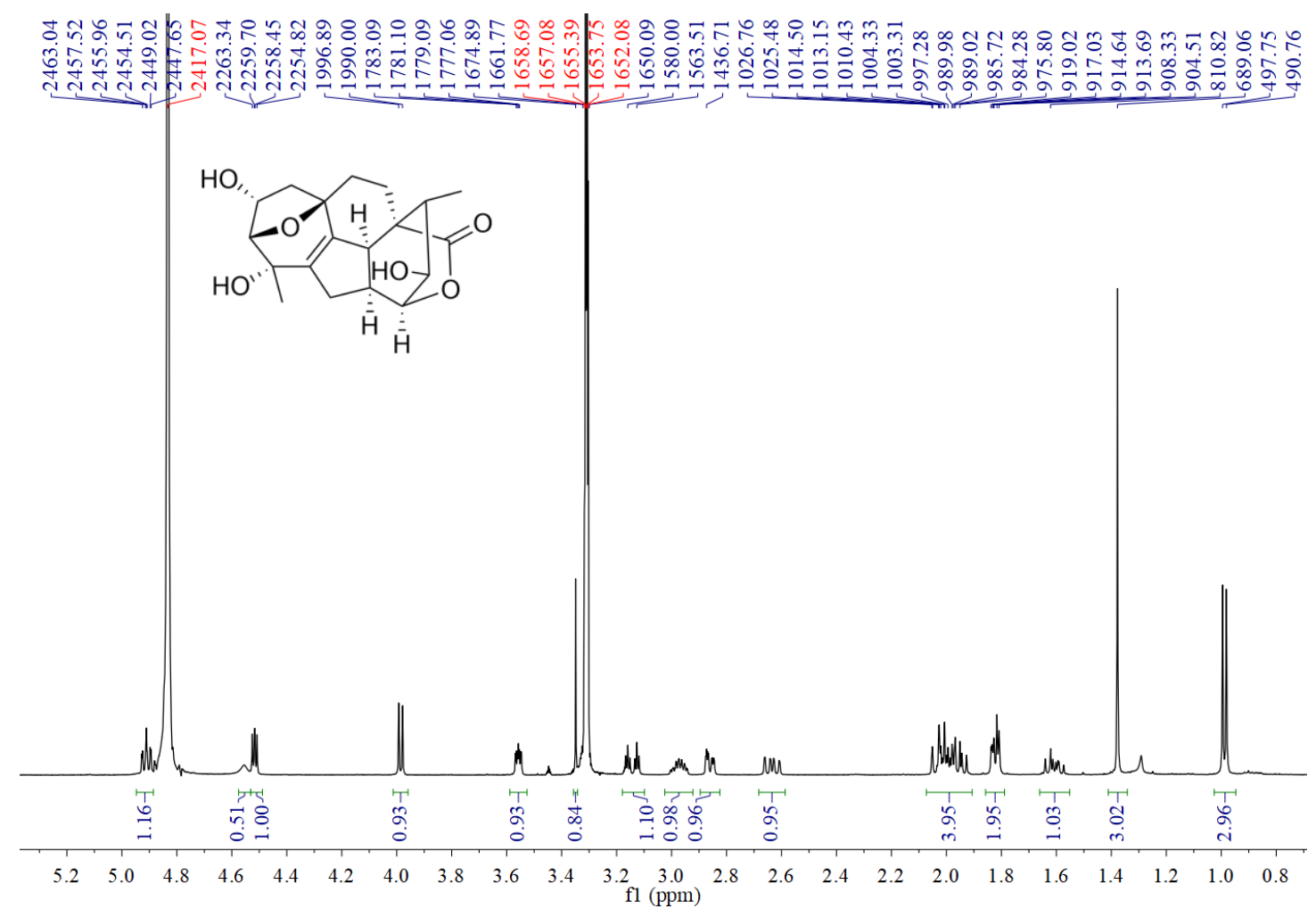


Figure S138. ${ }^{13} \mathrm{C}$ NMR spectrum of fortalpinoid L (12) in methanol- $d_{4}$.
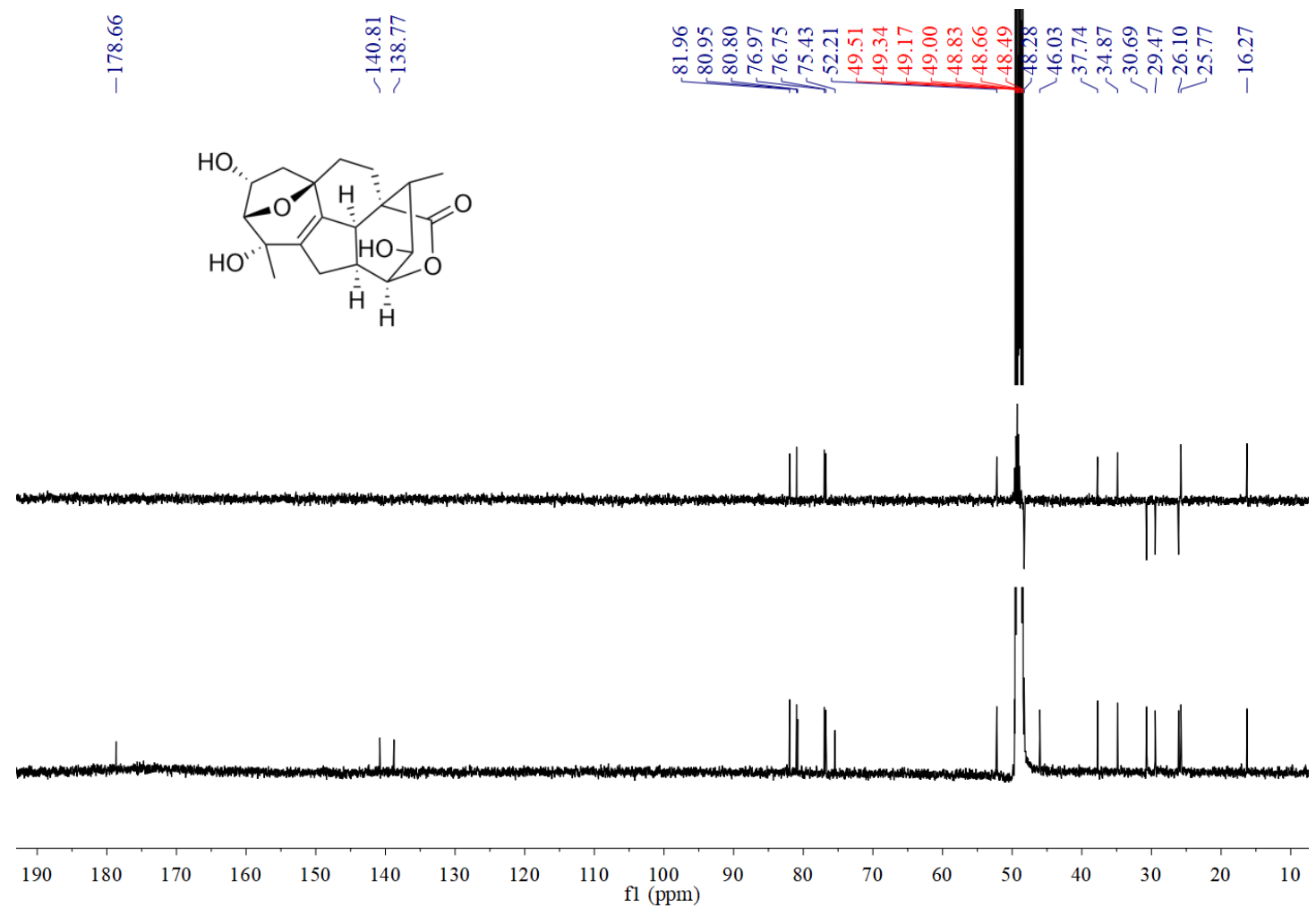

Figure S139. HSQC spectrum of fortalpinoid L (12) in methanol- $d_{4}$.

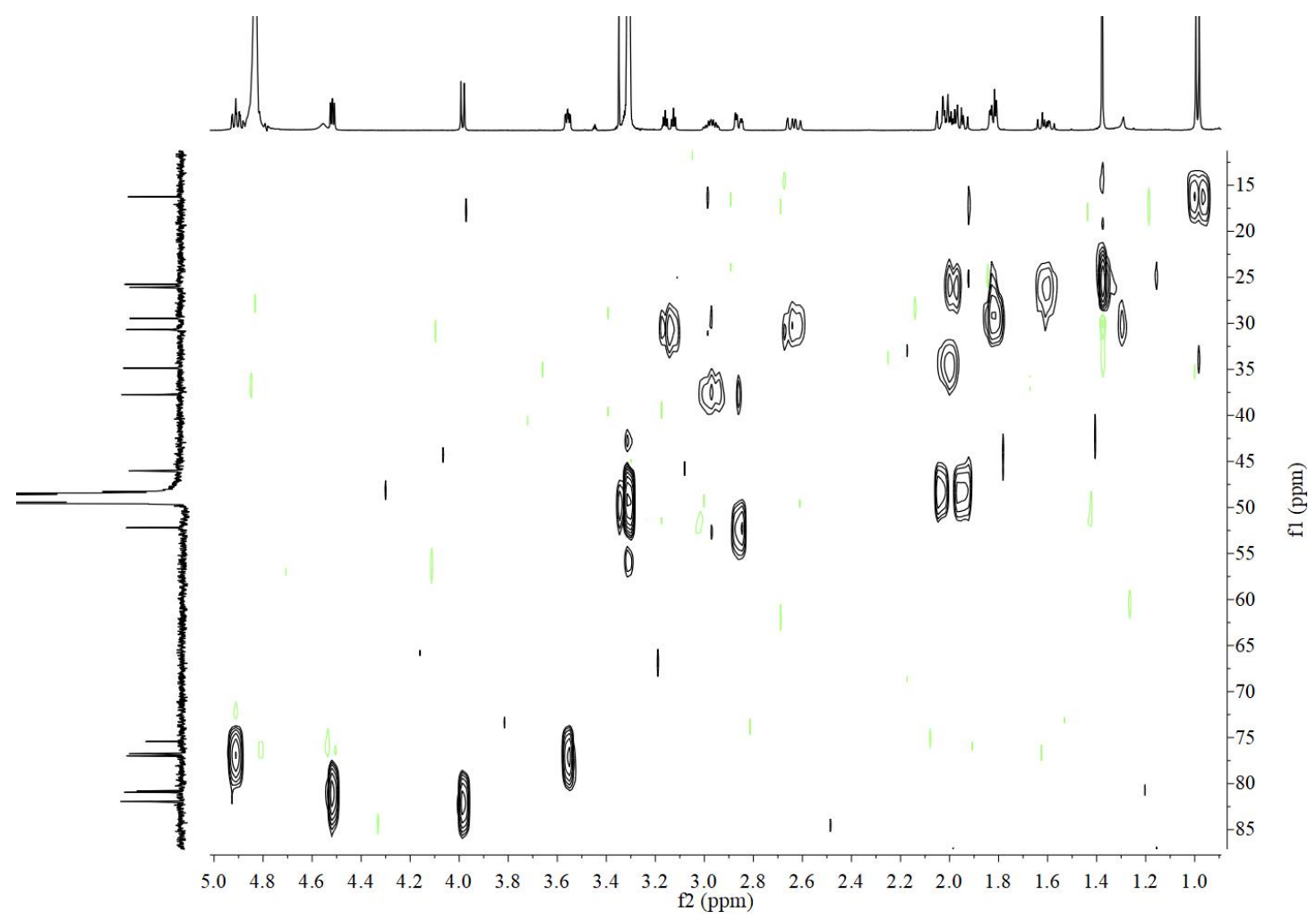


Figure S140. HMBC spectrum of fortalpinoid L (12) in methanol- $d_{4}$.

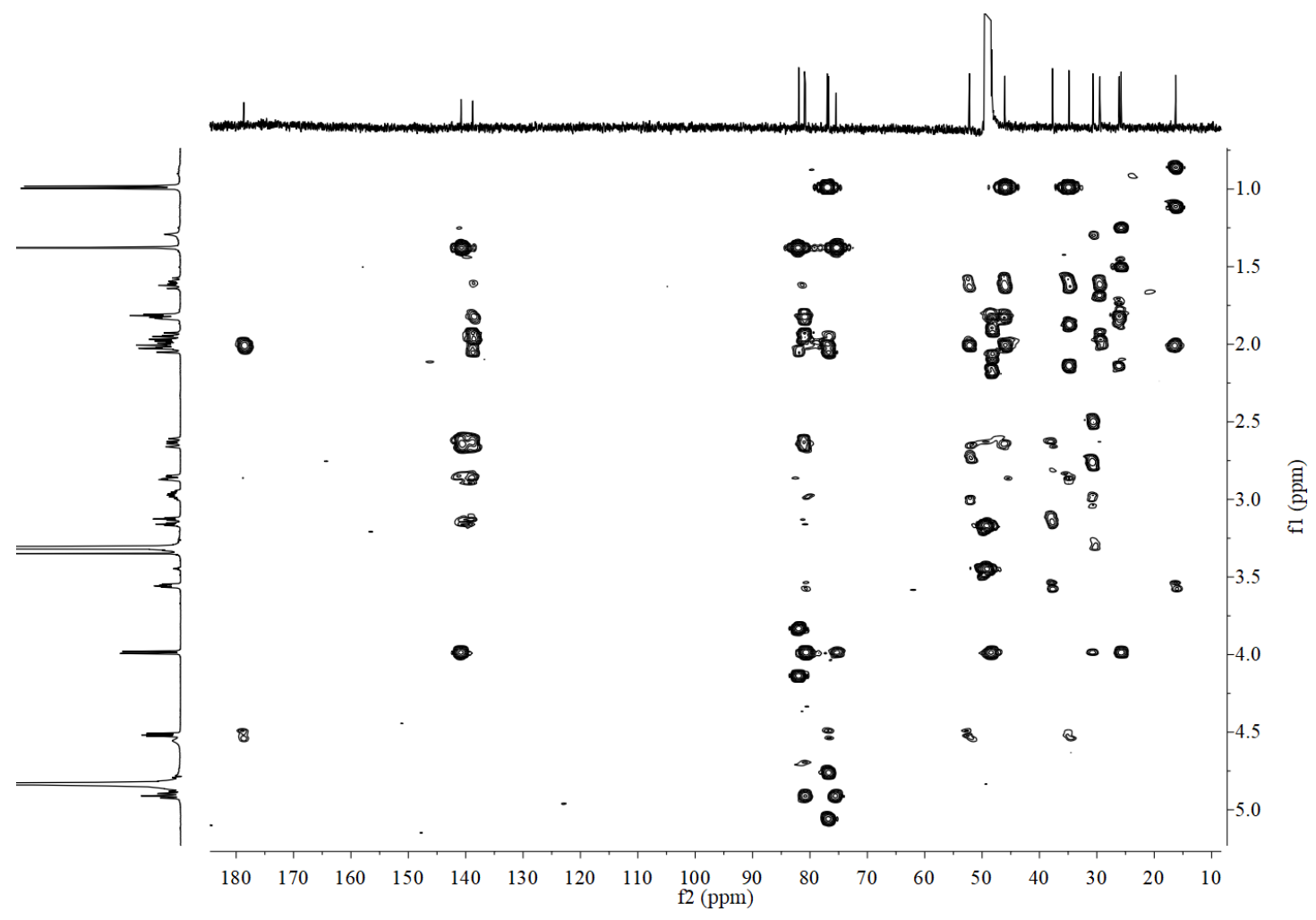

Figure S141. ${ }^{1} \mathrm{H}-{ }^{1} \mathrm{H}$ COSY spectrum of fortalpinoid L (12) in methanol- $d_{4}$.

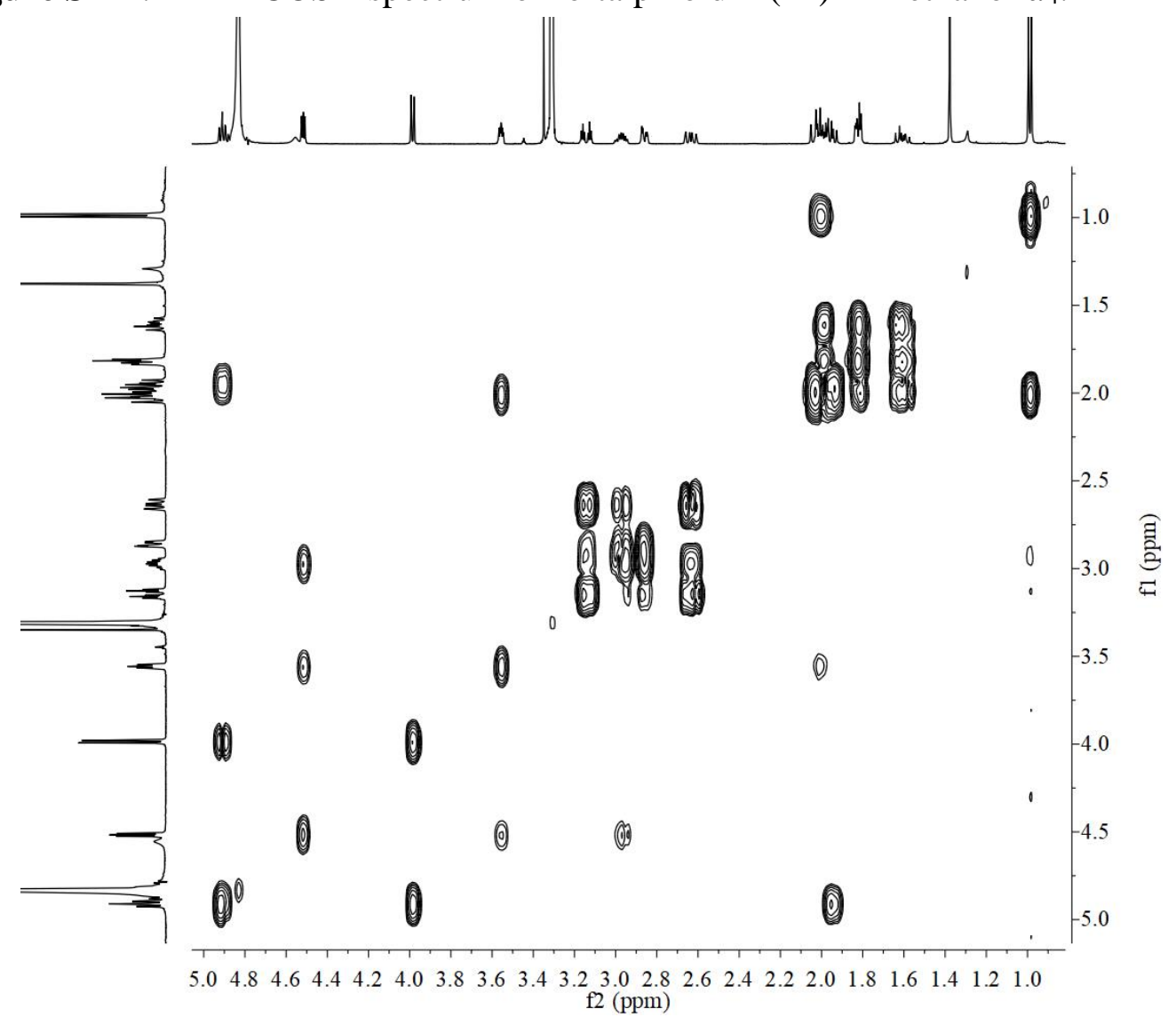


Figure S142. NOESY spectrum of fortalpinoid L (12) in methanol- $d_{4}$.

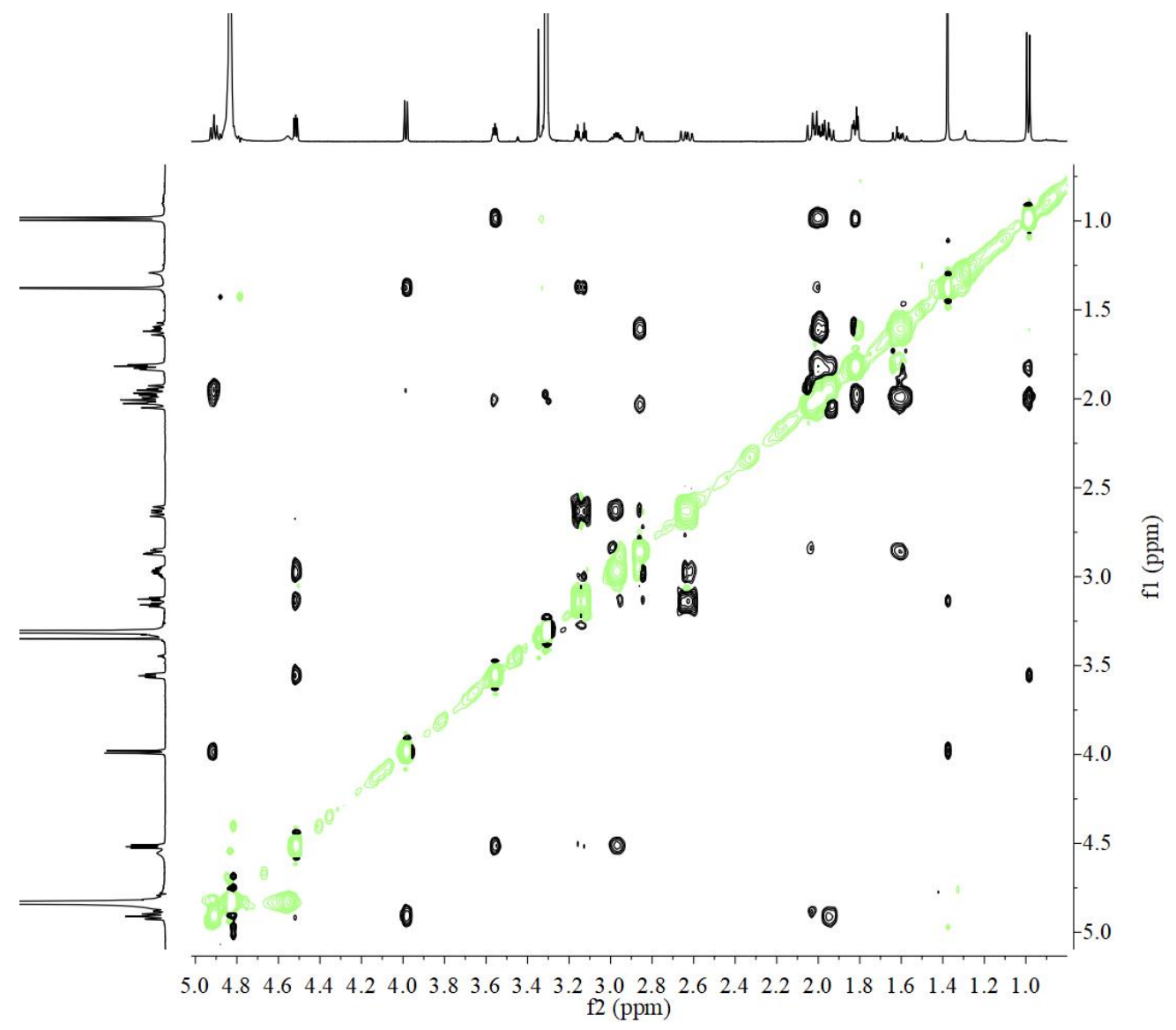

Figure S143. (-)-ESIMS spectrum of fortalpinoid L (12).

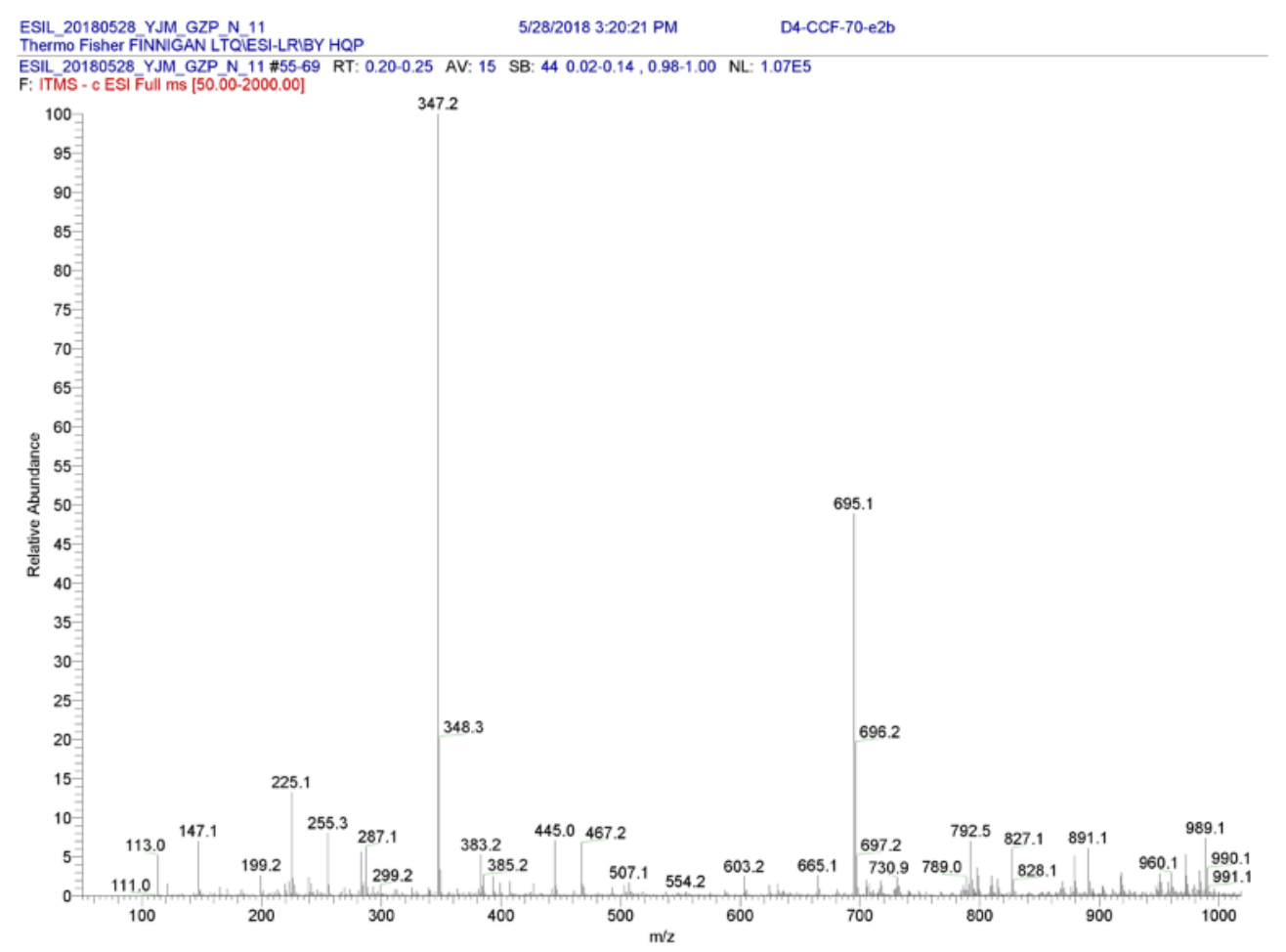


Figure S144. (-)-HRESIMS spectrum of fortalpinoid L (12).

Qualitative Analysis Report

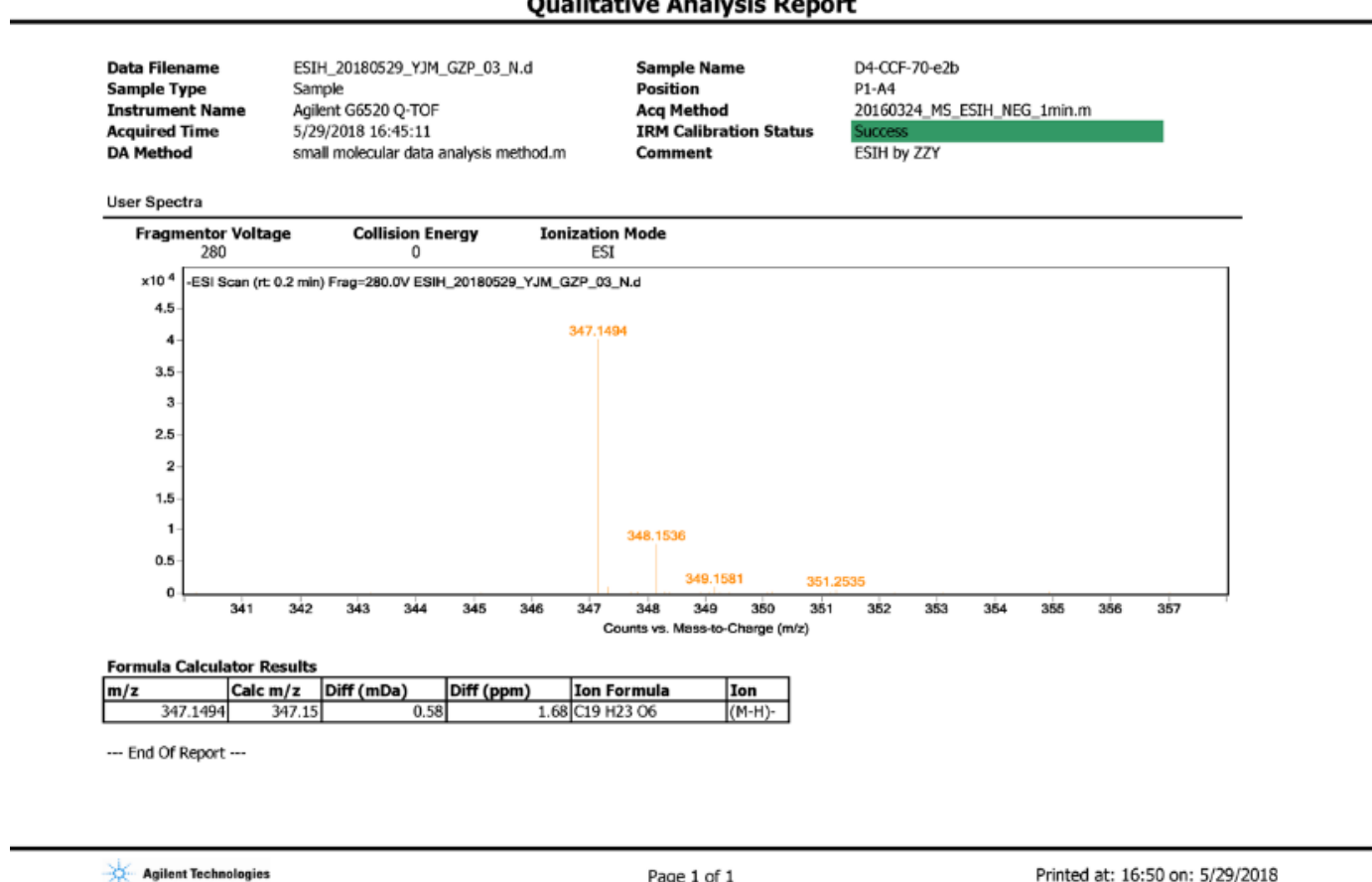

3र. Agilent Technologies

Page 1 of 1

Printed at: $16: 50$ on: $5 / 29 / 2018$

Figure S145. IR spectrum of fortalpinoid L (12).

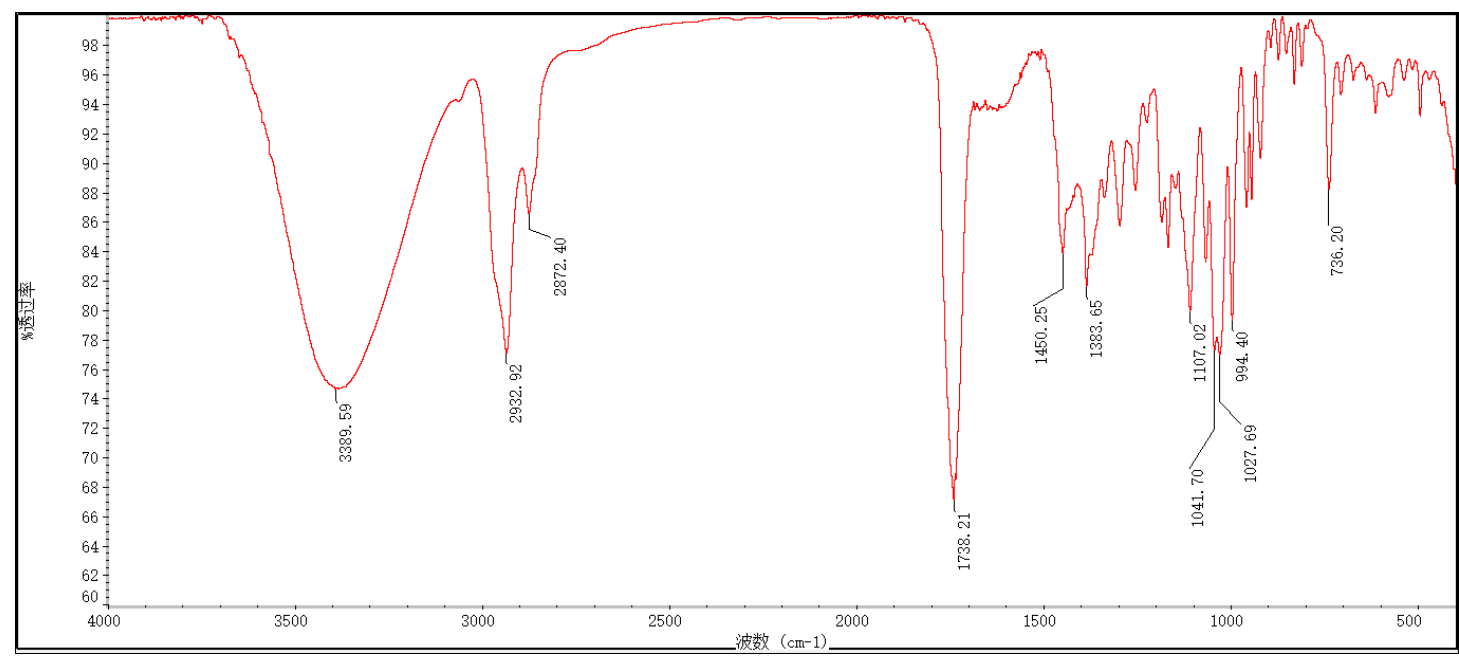


Figure S146. ECD spectra of fortalpinoid L (12).

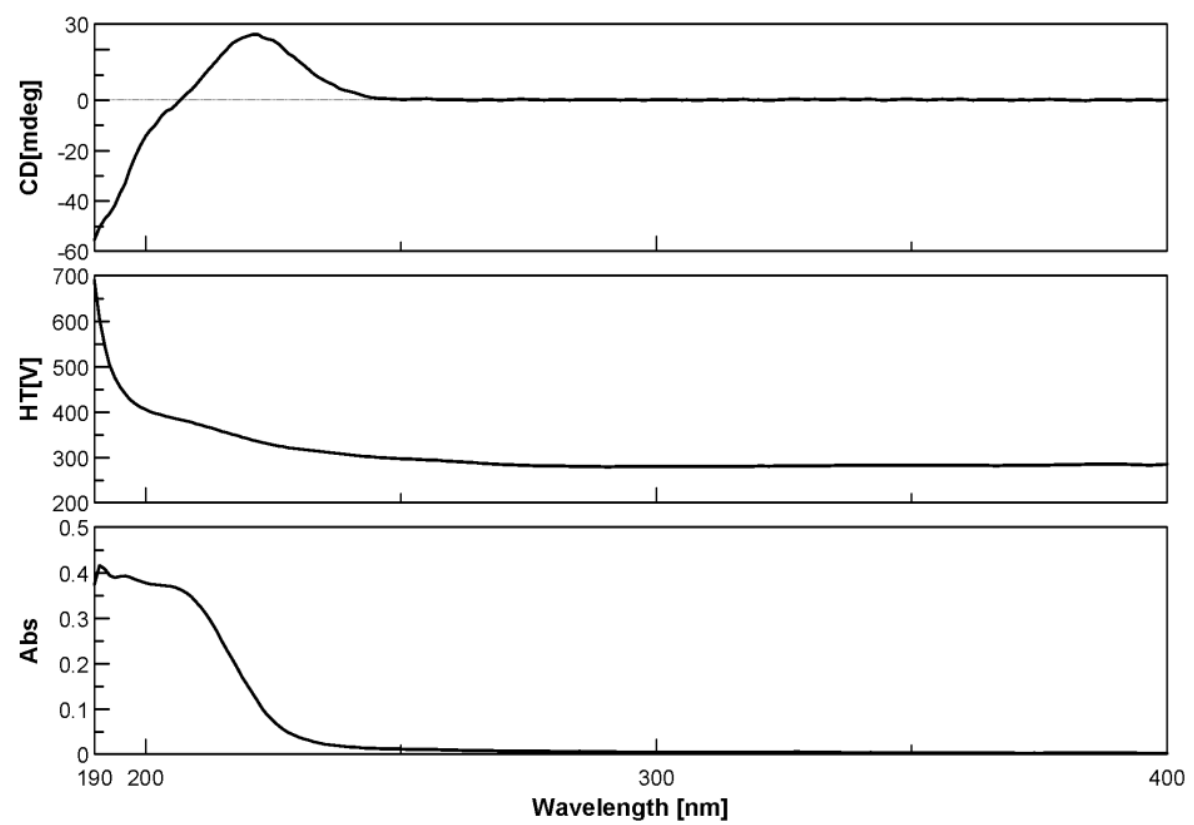

Figure S147. ${ }^{1} \mathrm{H}$ NMR spectrum of fortalpinoid $\mathrm{M}(13)$ in $\mathrm{CDCl}_{3}$.

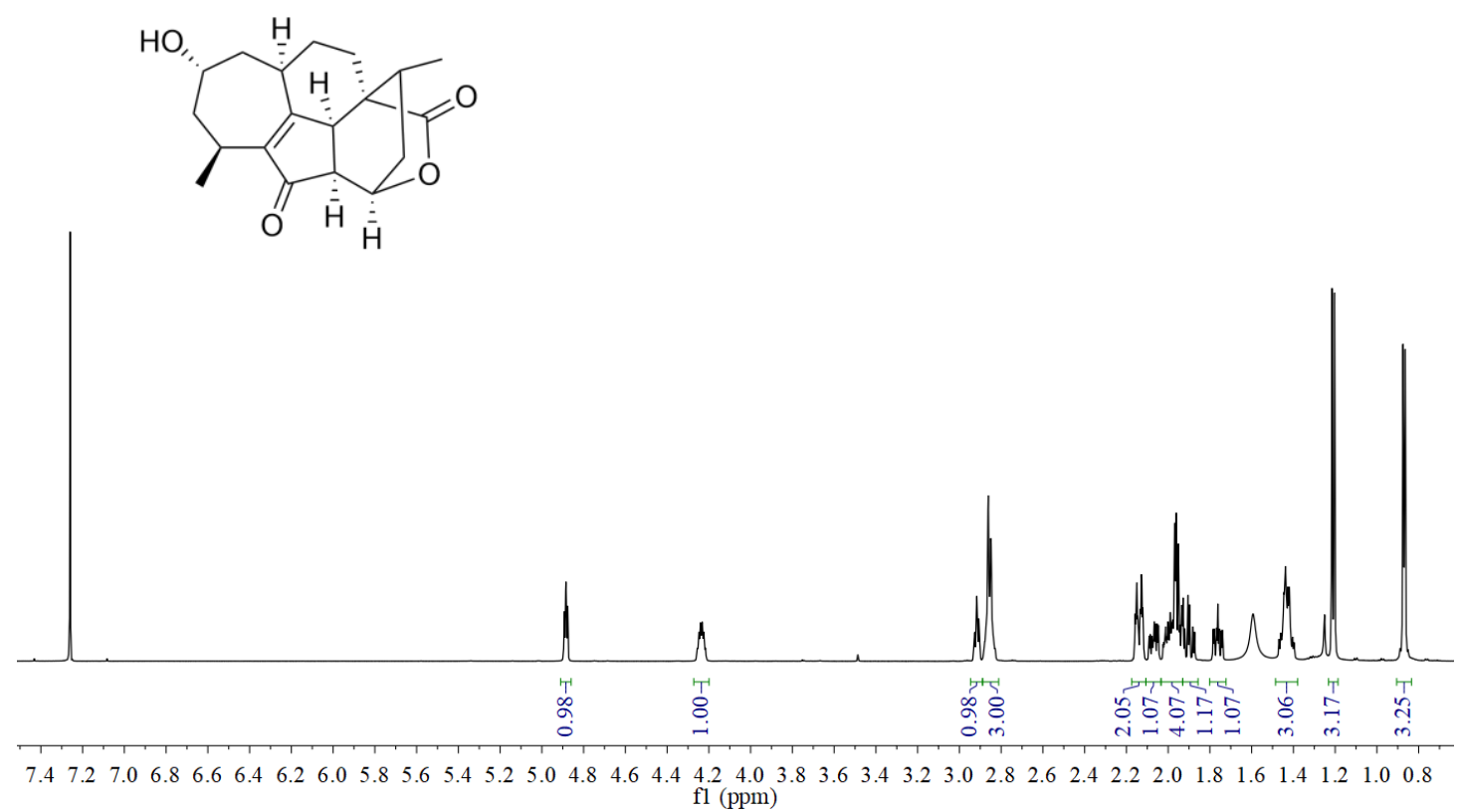


Figure S148. ${ }^{13} \mathrm{C}$ NMR spectrum of fortalpinoid $\mathrm{M}(13)$ in $\mathrm{CDCl}_{3}$.
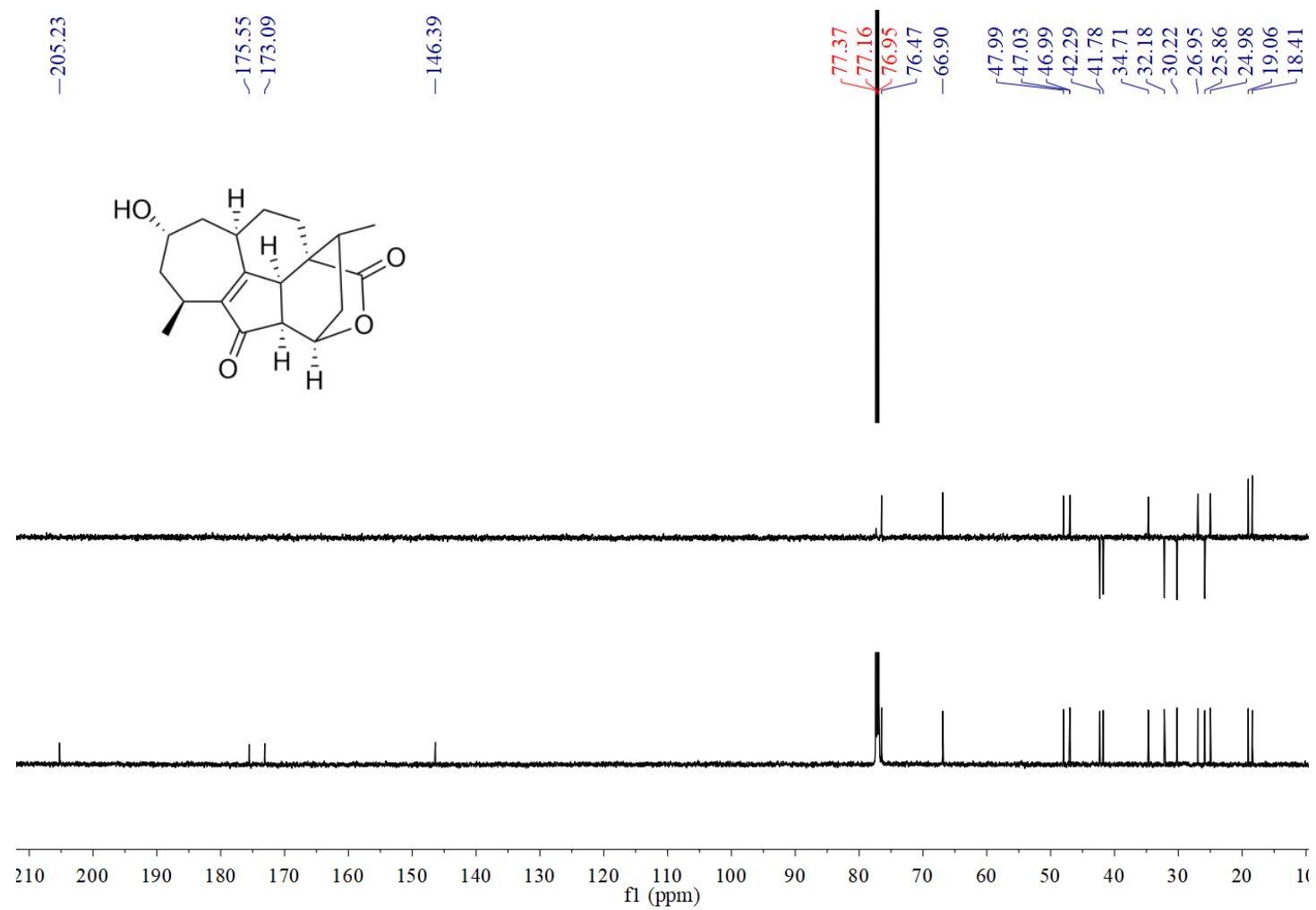

Figure S149. HSQC spectrum of fortalpinoid $\mathrm{M}(\mathbf{1 3})$ in $\mathrm{CDCl}_{3}$.

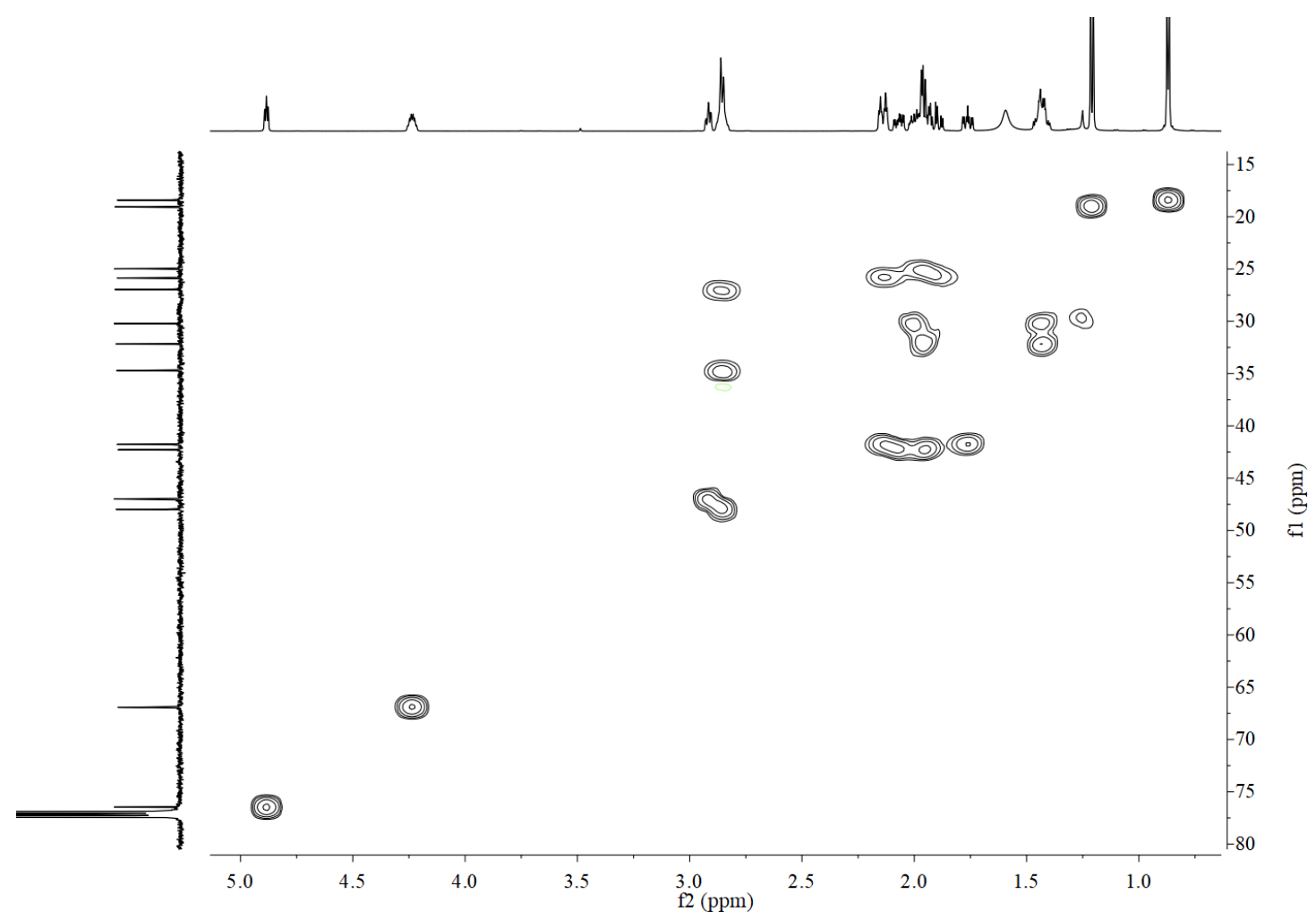


Figure S150. HMBC spectrum of fortalpinoid $\mathrm{M}(13)$ in $\mathrm{CDCl}_{3}$.

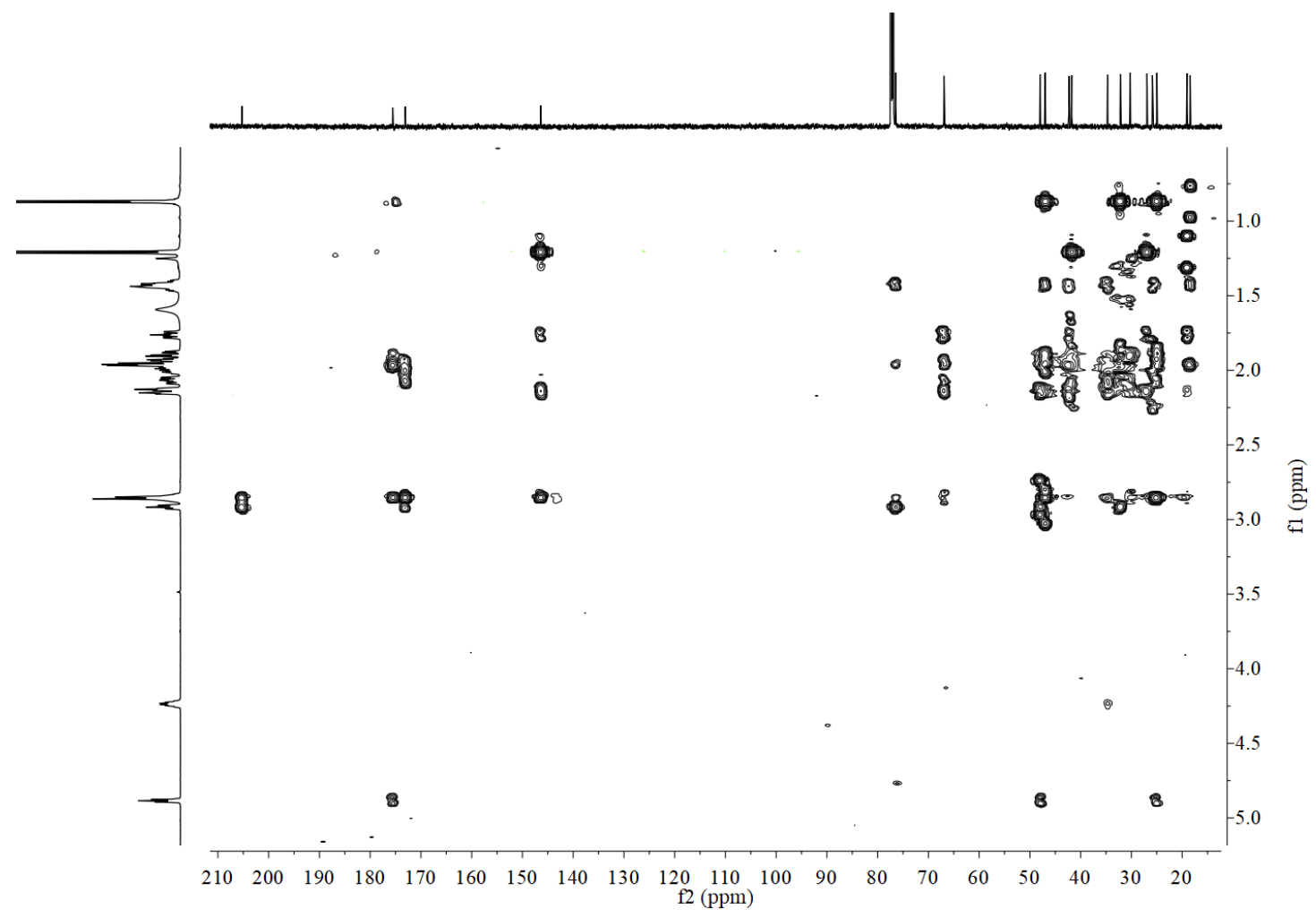

Figure S151. ${ }^{1} \mathrm{H}-{ }^{1} \mathrm{H}$ COSY spectrum of fortalpinoid $\mathrm{M}(\mathbf{1 3})$ in $\mathrm{CDCl}_{3}$.

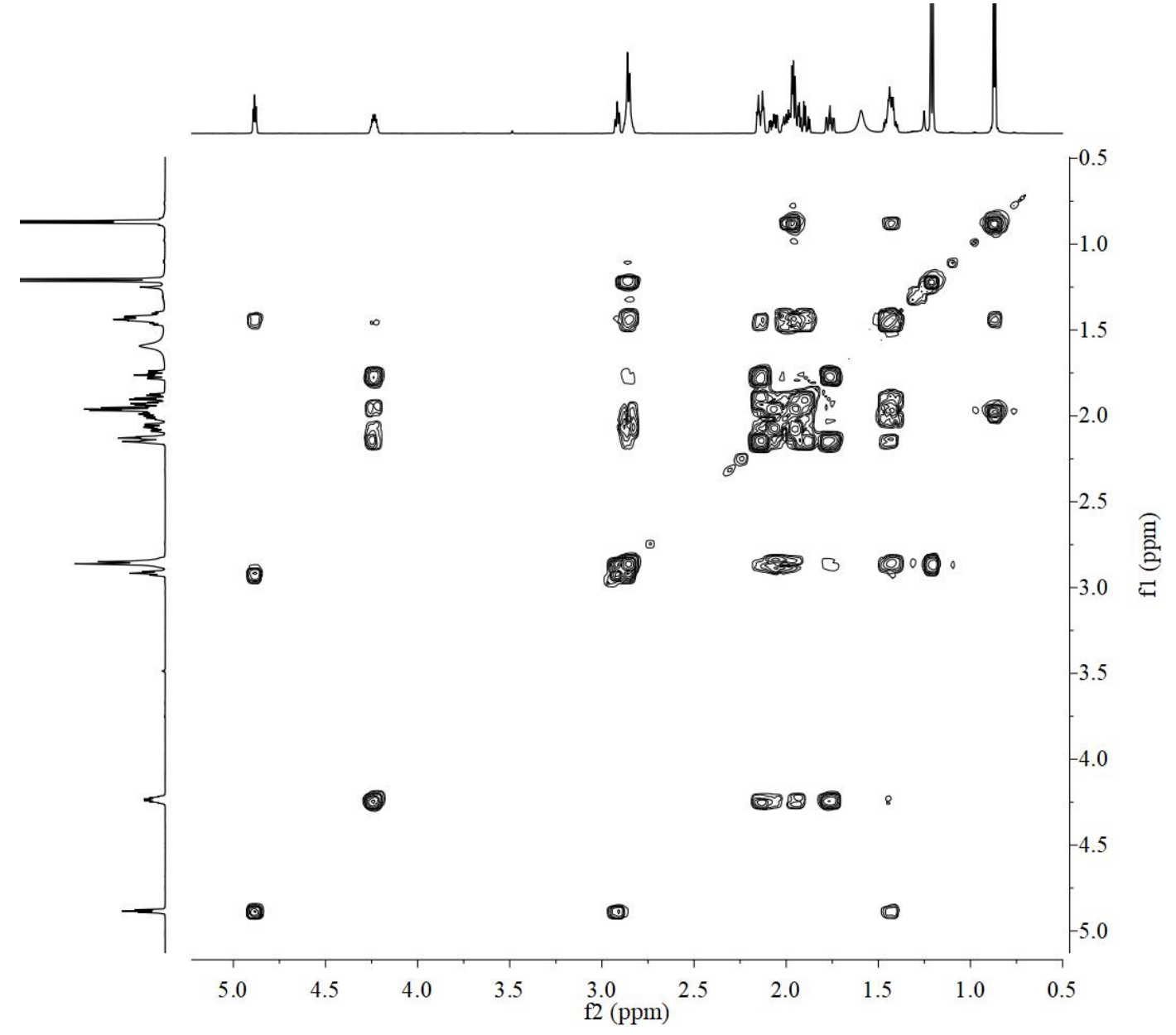


Figure S152. NOESY spectrum of fortalpinoid $\mathrm{M}(\mathbf{1 3})$ in $\mathrm{CDCl}_{3}$.

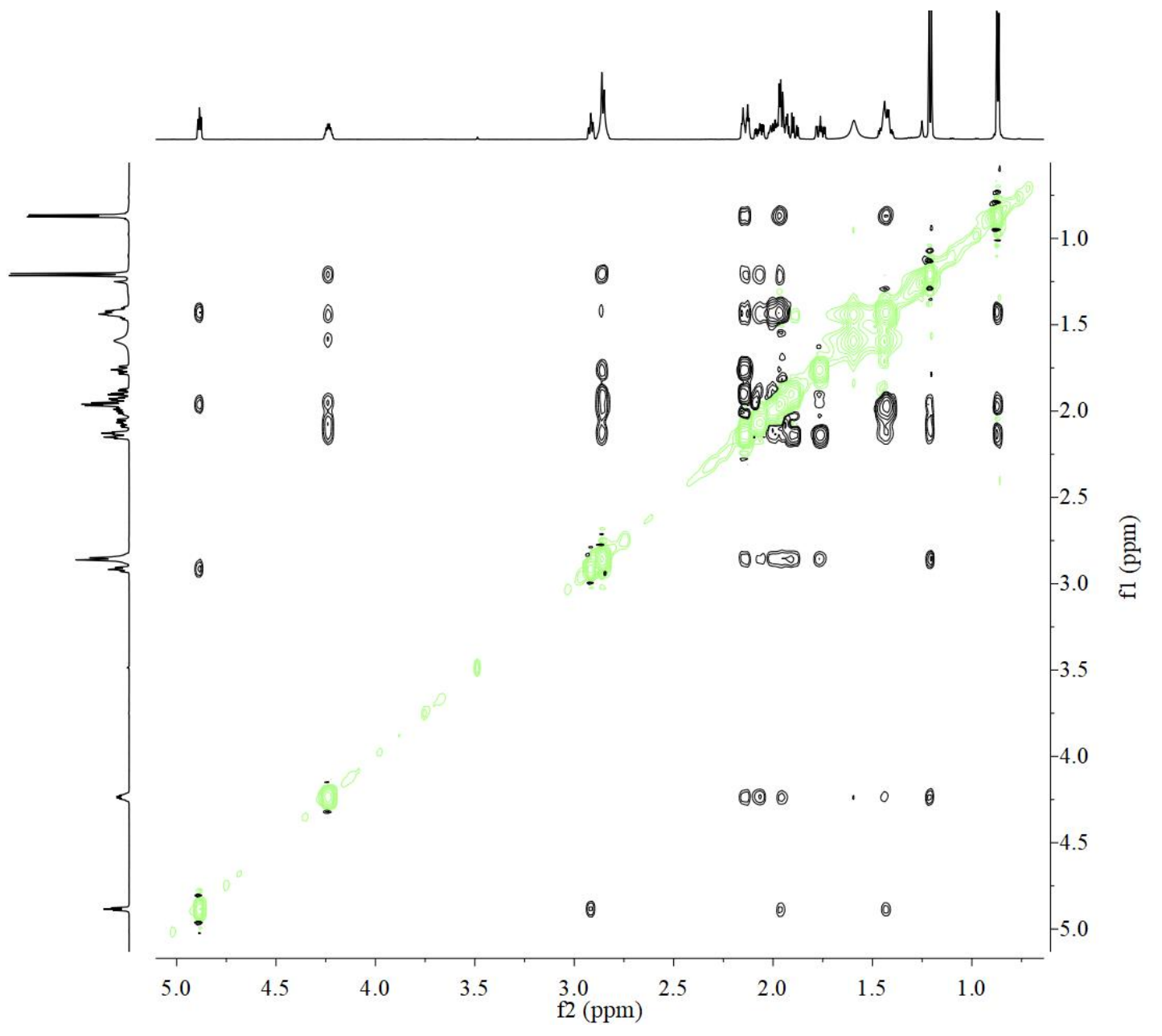

Figure S153. (+)-ESIMS spectrum of fortalpinoid M (13).

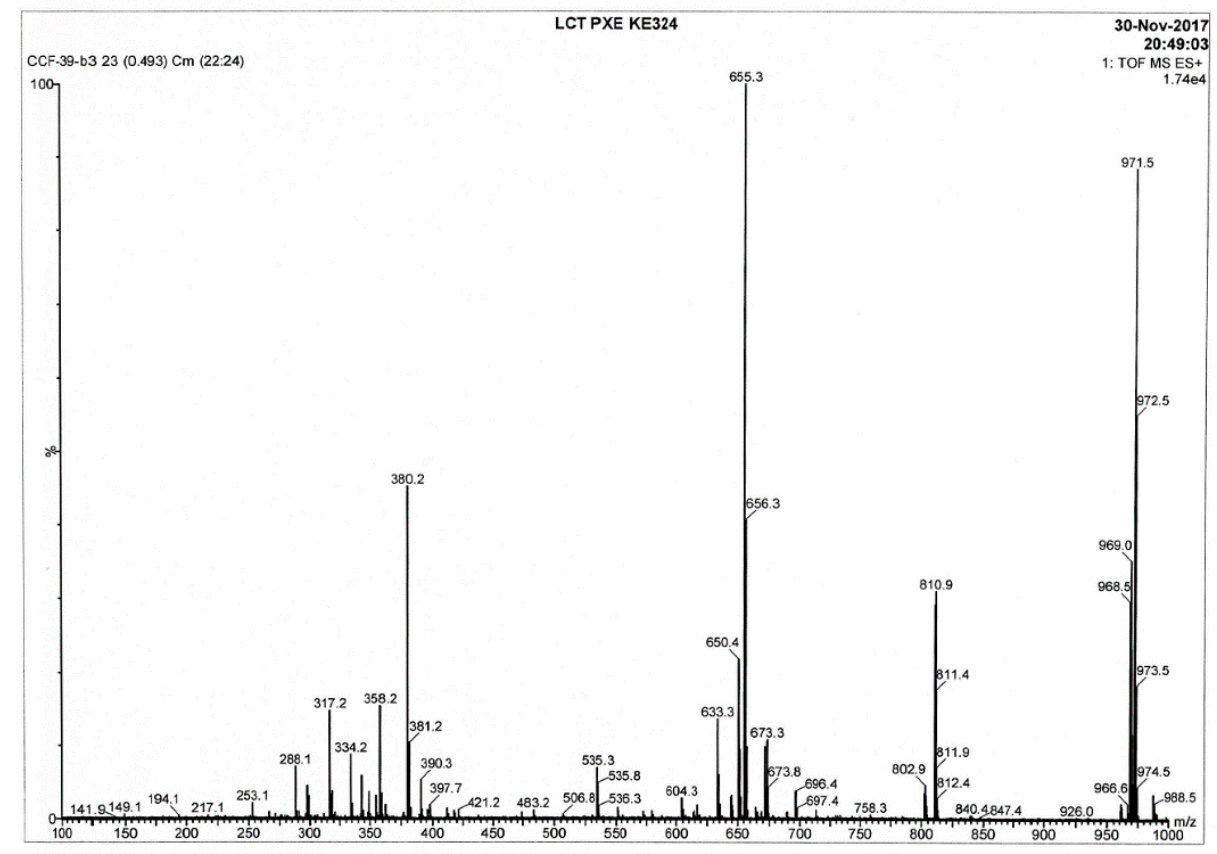


Figure S154. (-)-ESIMS spectrum of fortalpinoid M (13).

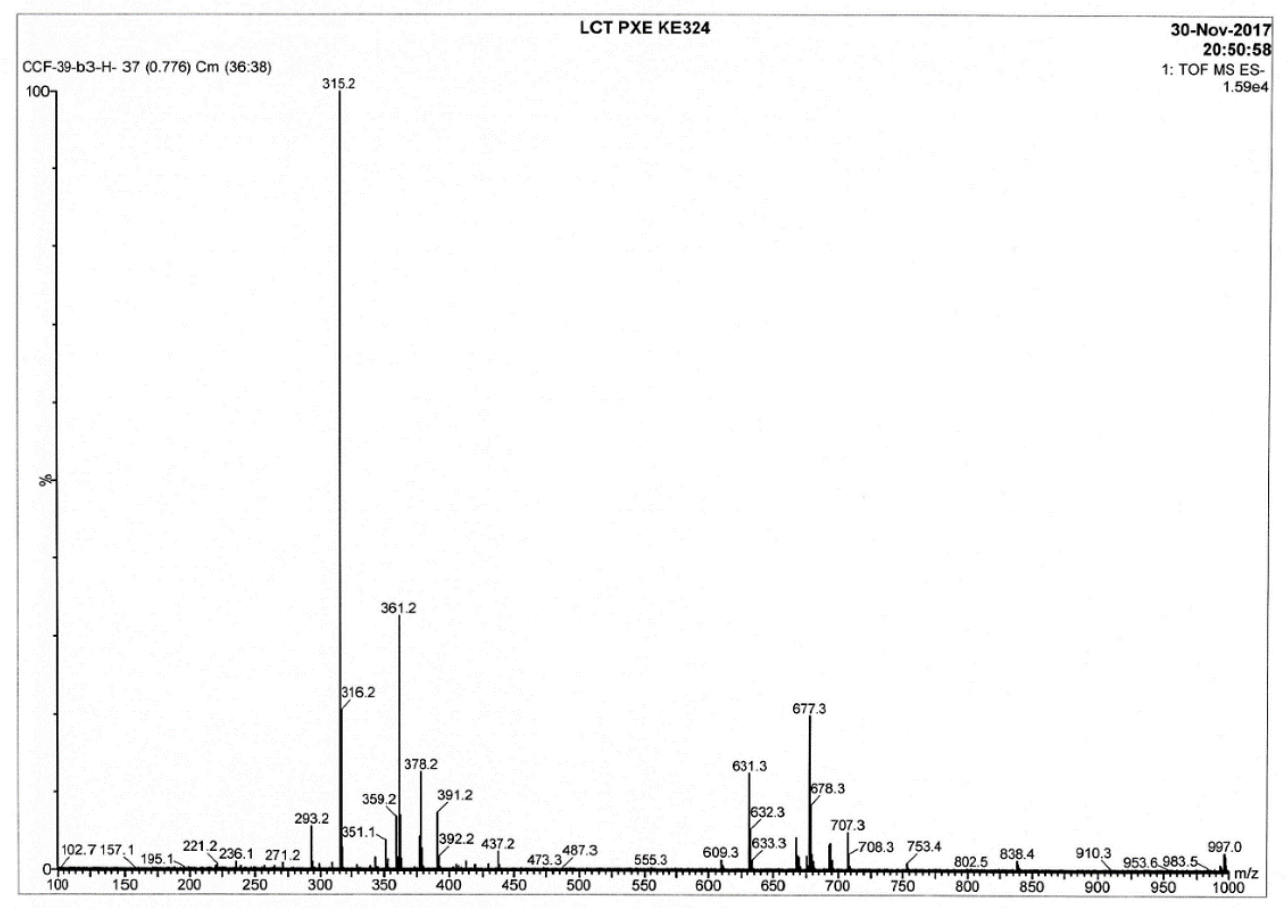

Figure S155. (-)-HRESIMS spectrum of fortalpinoid M (13).

Elomontal Composition Report

Page 1

Single Mass Analysis

Tolerance $=3.0$ PPM I DBE: $\min =-1.5, \max =50.0$

Element prediction: Off

Number of isotope peaks used for $\mathrm{i}-\mathrm{FIT}=3$

Monoisotopic Mass, Even Electron lons

113 formula(e) evaluated with 1 results within limits (up to 50 best isotopic matches for each mass) Elements Used:

$\begin{array}{llll}C: & 0-100 & \text { H: } 0-1000 & 0: 0-500 \quad \mathrm{Na}: 0-1\end{array}$

LCT PXE KE324

30-Nov-2017

CCF-39-b3-H- 92 (1.993) $\begin{array}{r}20: 50: 58 \\ \text { Cm (92:93) }\end{array}$

1: TOF MS ES.

(3)

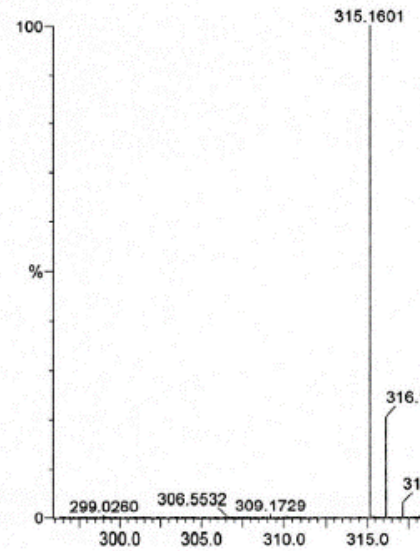

$1.03 \mathrm{e}+004$

Minimum:

Maximum:

Mass 5.

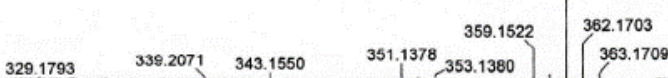
$300.0305 .0 \quad 310.0$

-1.5
50.0 Calc. Mass mDa PPM

DBE i-FIT

i-FIT (Norm) Formula

315.1596

0.5

1.6

8.5

139.7

0.0

$\begin{array}{lll}\text { C19 } & \text { H23 } & \text { O4 }\end{array}$ 
Figure S156. IR spectrum of fortalpinoid M (13).

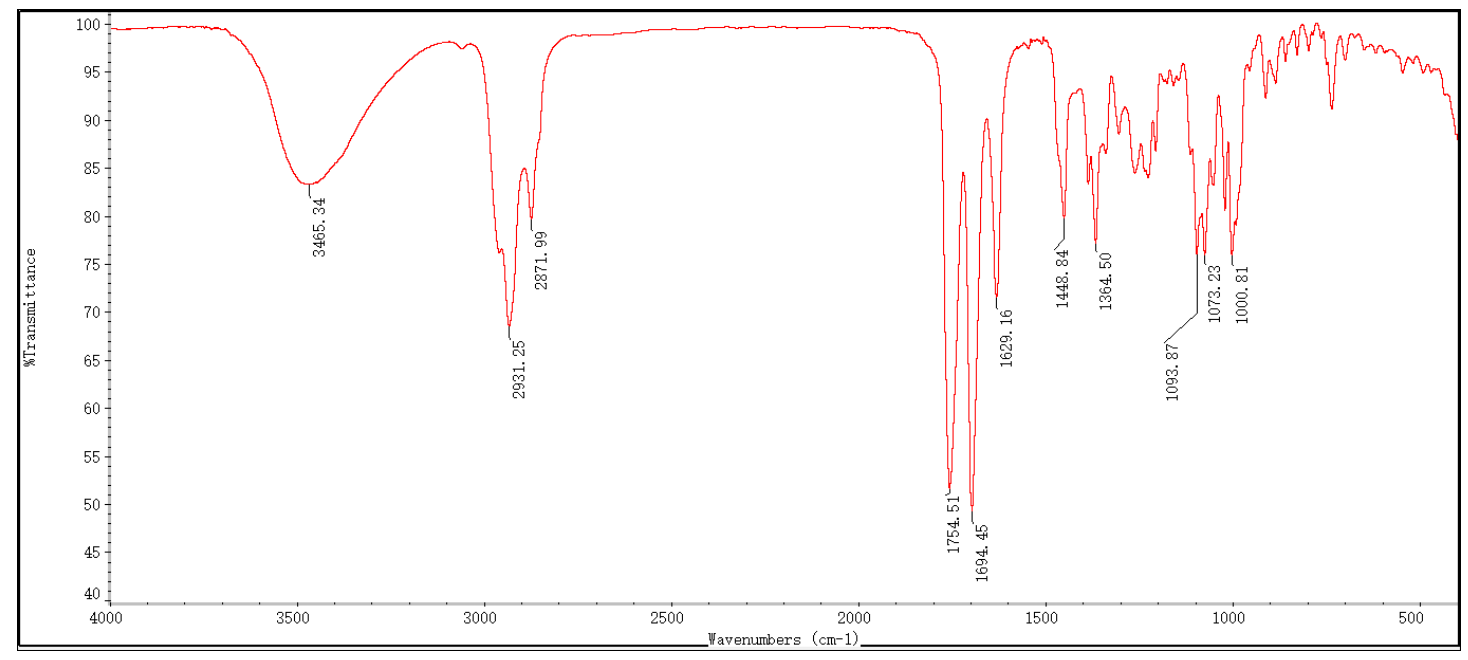

Figure S157. ${ }^{1} \mathrm{H}$ NMR spectrum of fortalpinoid $\mathrm{N}(14)$ in $\mathrm{CDCl}_{3}$.

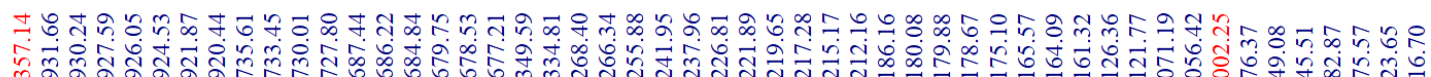

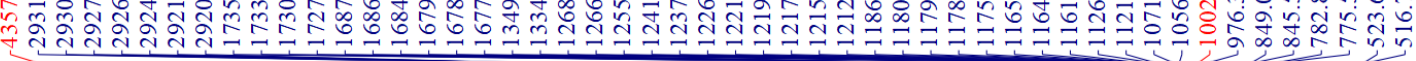

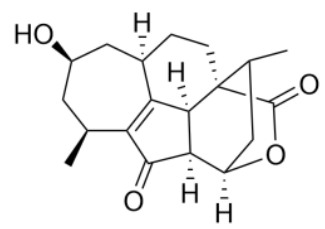

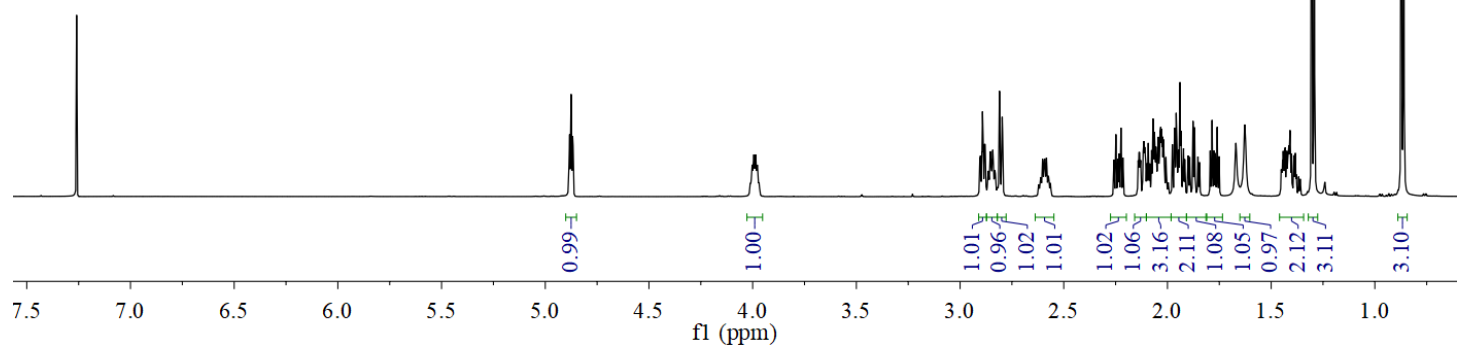


Figure S158. ${ }^{13} \mathrm{C}$ NMR spectrum of fortalpinoid $\mathrm{N}(\mathbf{1 4})$ in $\mathrm{CDCl}_{3}$.
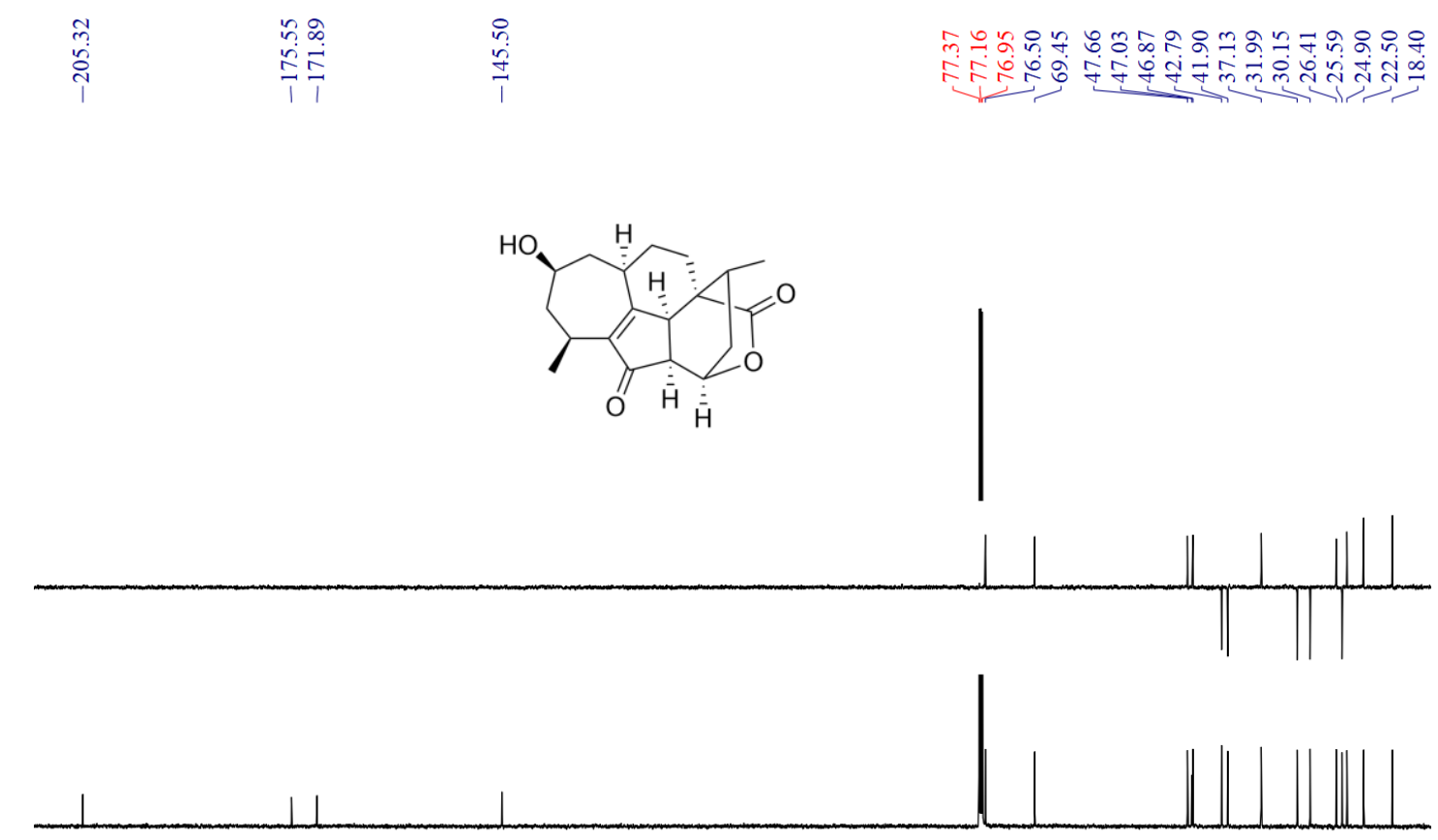

Figure S159. HSQC spectrum of fortalpinoid N (14) in $\mathrm{CDCl}_{3}$.

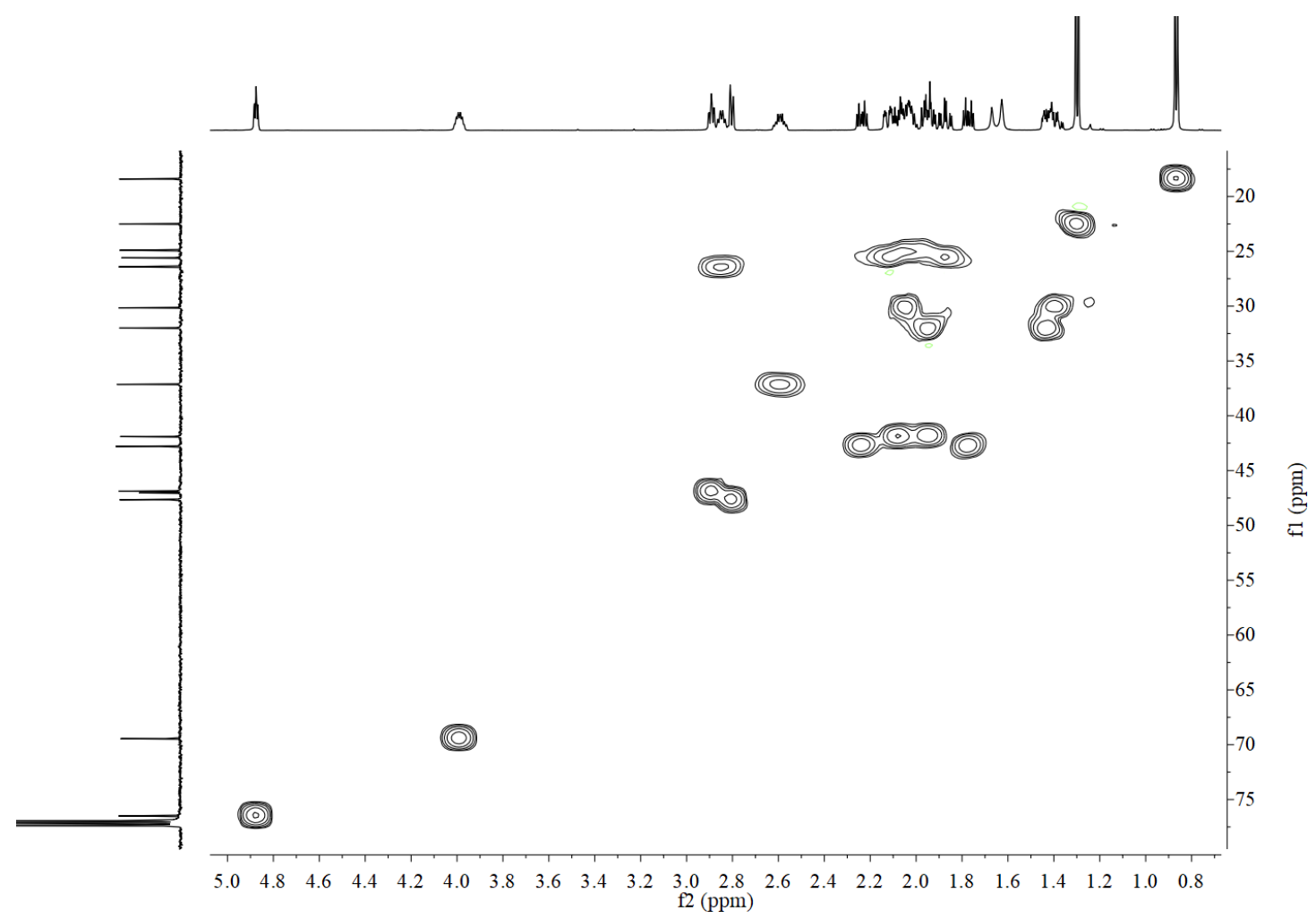


Figure S160. HMBC spectrum of fortalpinoid $\mathrm{N}(14)$ in $\mathrm{CDCl}_{3}$.

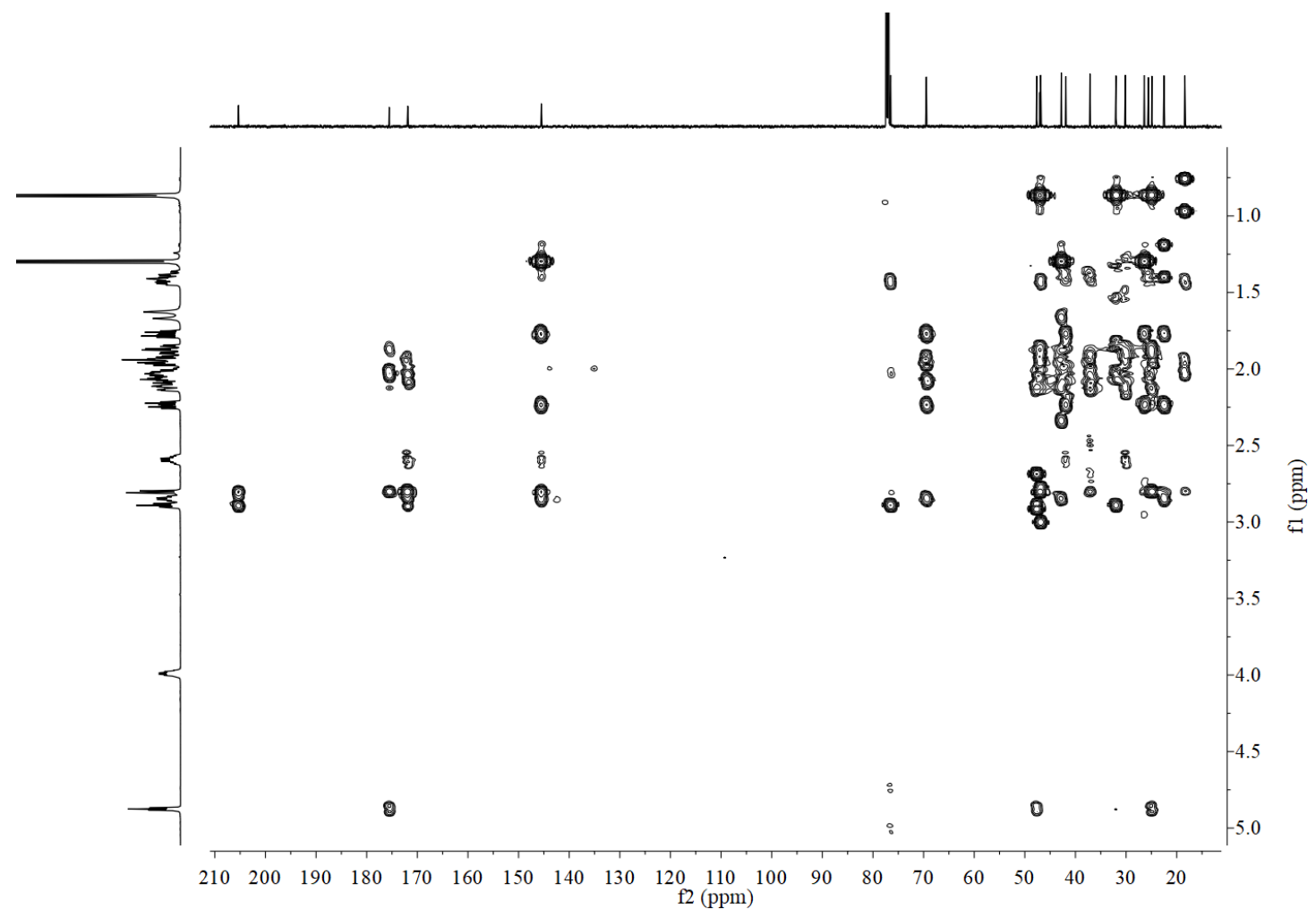

Figure S161. ${ }^{1} \mathrm{H}-{ }^{1} \mathrm{H}$ COSY spectrum of fortalpinoid $\mathrm{N}(\mathbf{1 4})$ in $\mathrm{CDCl}_{3}$.

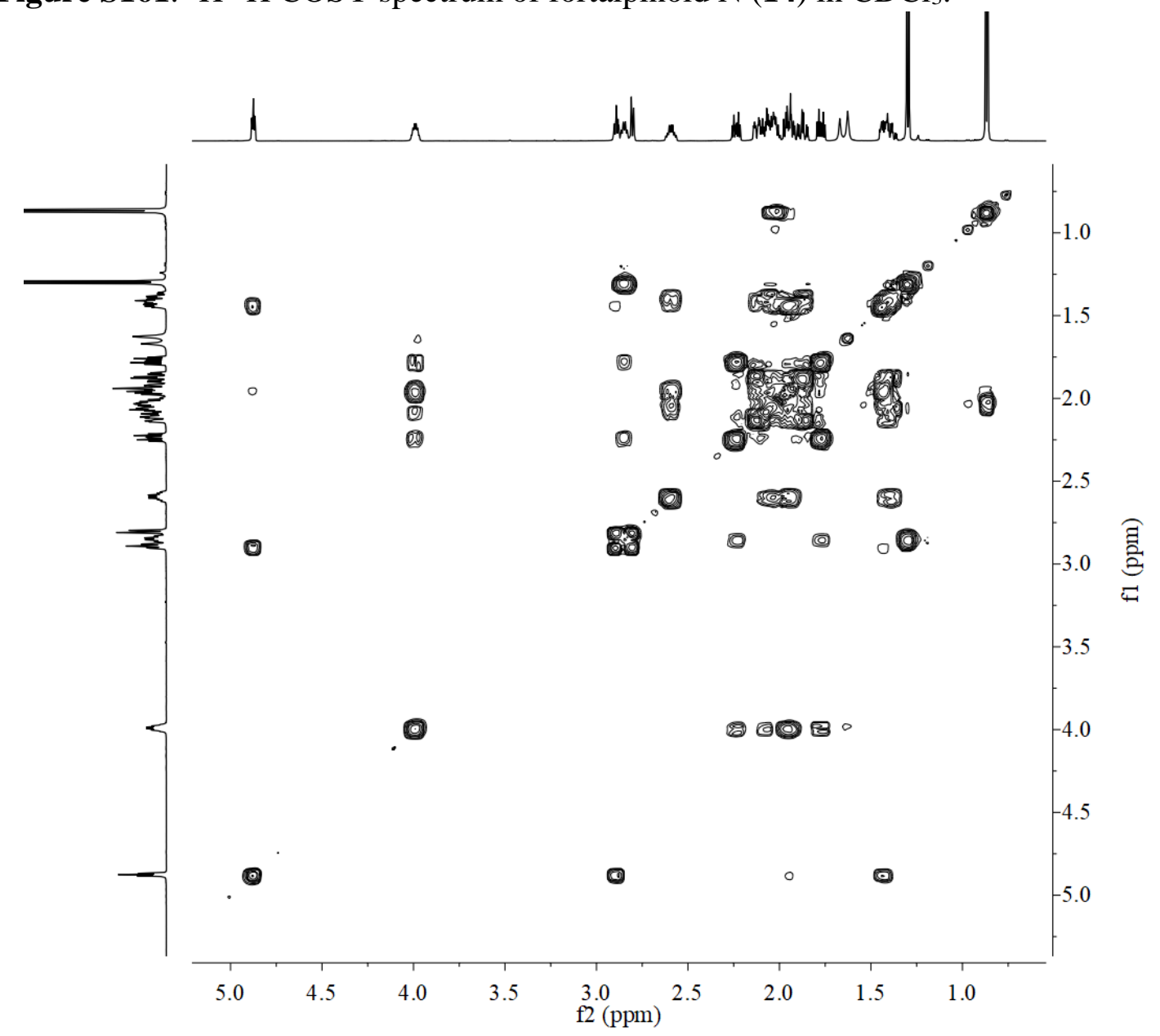


Figure S162. NOESY spectrum of fortalpinoid $\mathrm{N}(14)$ in $\mathrm{CDCl}_{3}$.

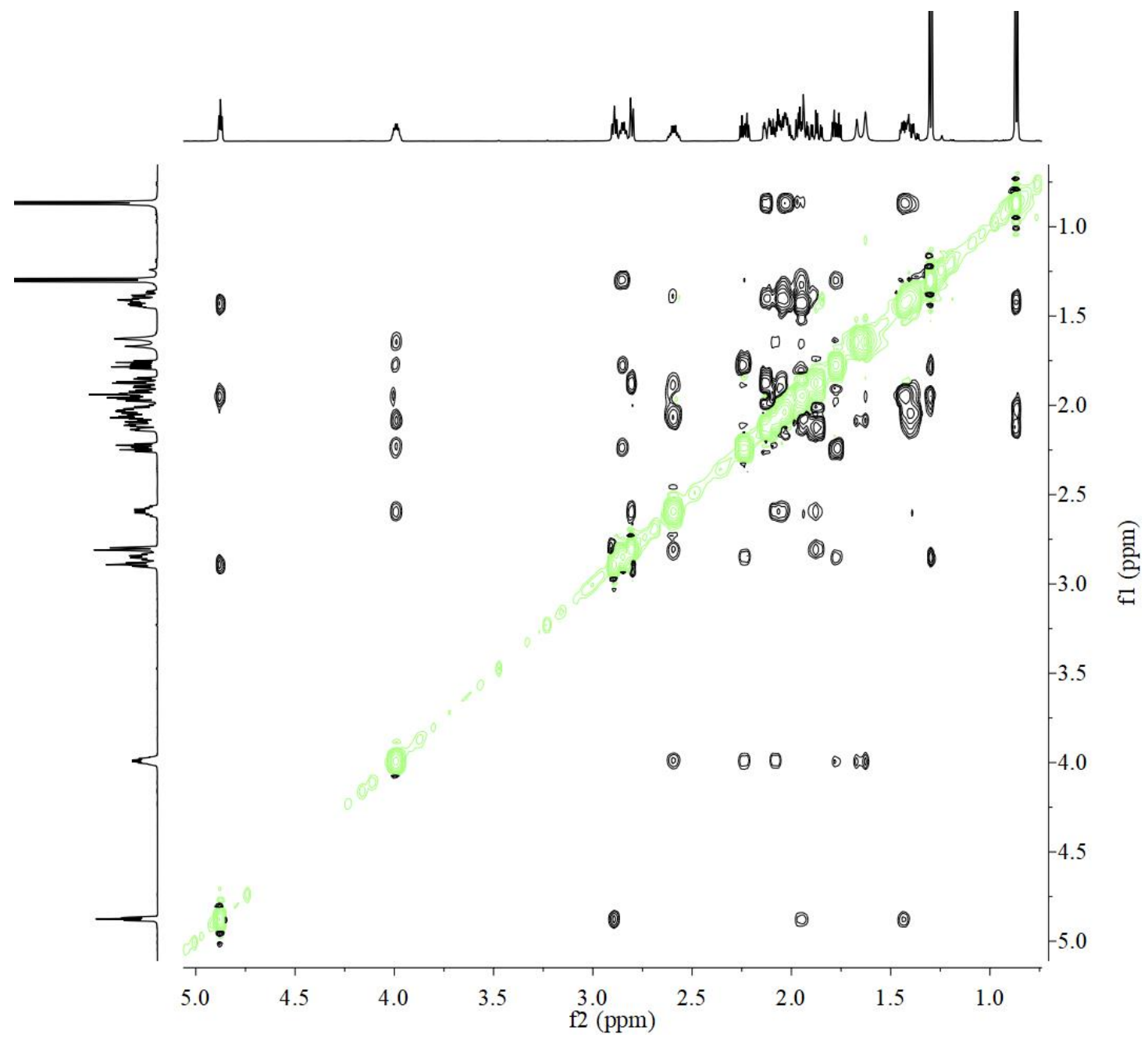

Figure S163. (+)-ESIMS spectrum of fortalpinoid N (14).

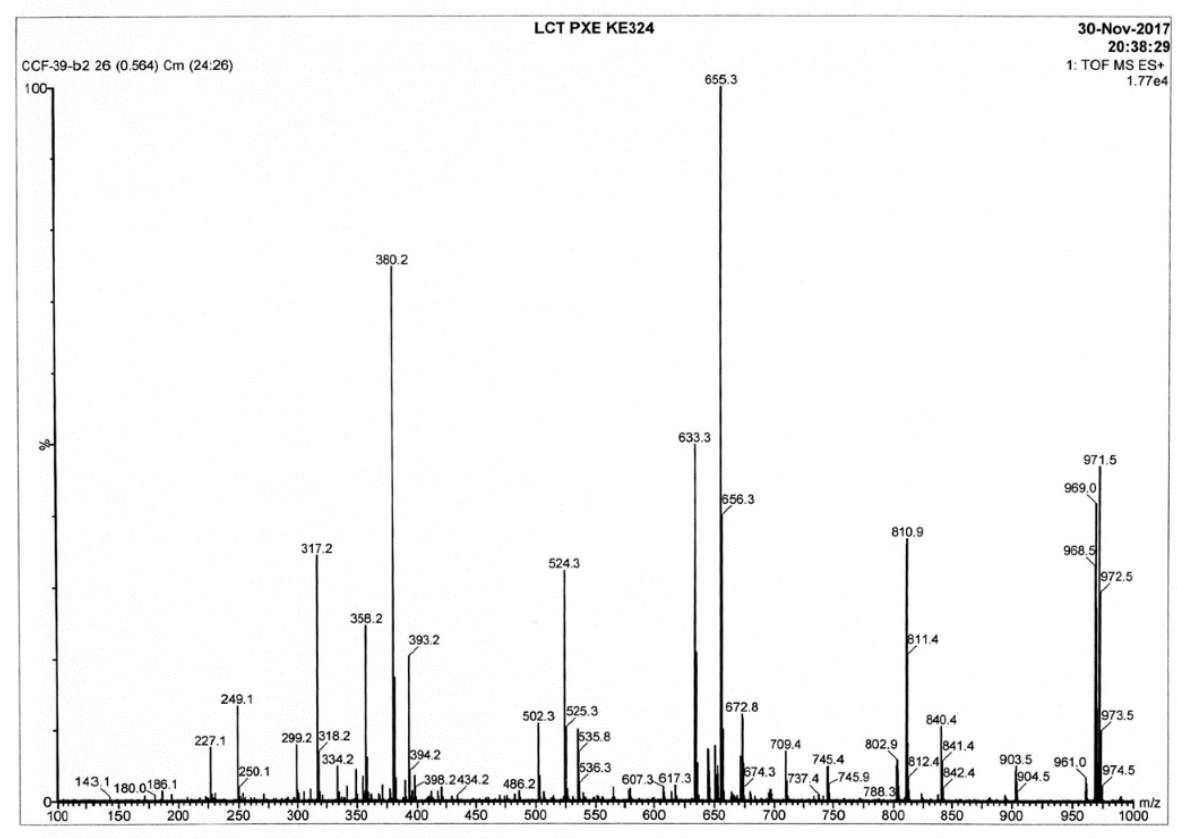


Figure S164. (-)-ESIMS spectrum of fortalpinoid N (14).

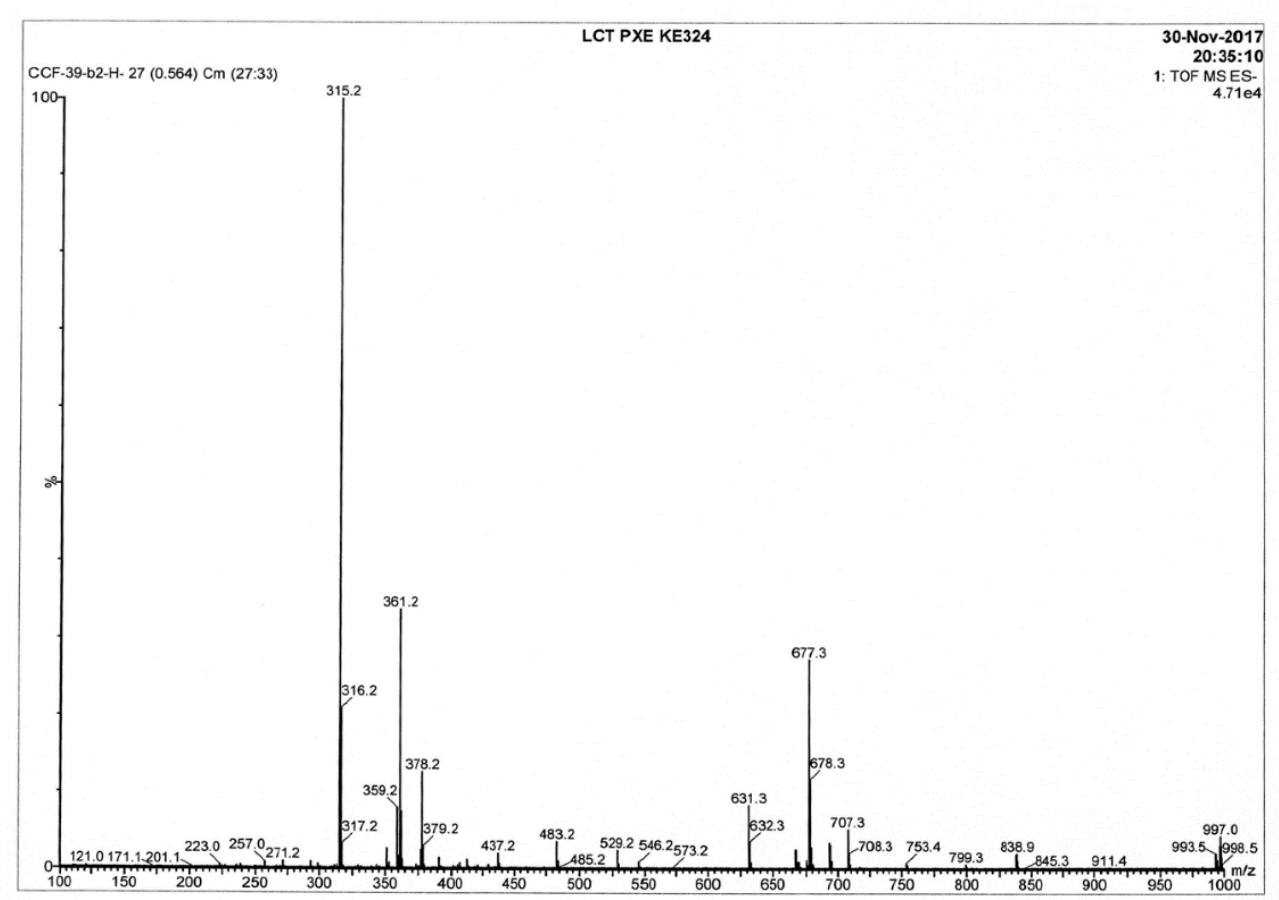

Figure S165. (-)-HRESIMS spectrum of fortalpinoid N (14).

Elemental Composition Report

Page 1

Single Mass Analysis

Tolerance $=3.0$ PPM I DBE: $\min =-1.5, \max =50.0$

Element prediction: Off

Monoisotopic Mass, Even Electron lons

113 formula(e) evaluated with 1 results within limits (up to 50 best isotopic matches for each mass)

$\begin{array}{llll}\text { Elements Used: } 0-100 \quad \text { H: } 0-1000 & 0: 0-500 \quad \mathrm{Na}: 0-1\end{array}$

LCT PXE KE324

30-Nov-2017

1: TOF MS ES.

CCF-39-b2-H-70 (1.516) ${ }^{20-35: 10}(70)$

${ }^{100}$

315.1596

6.19e+003
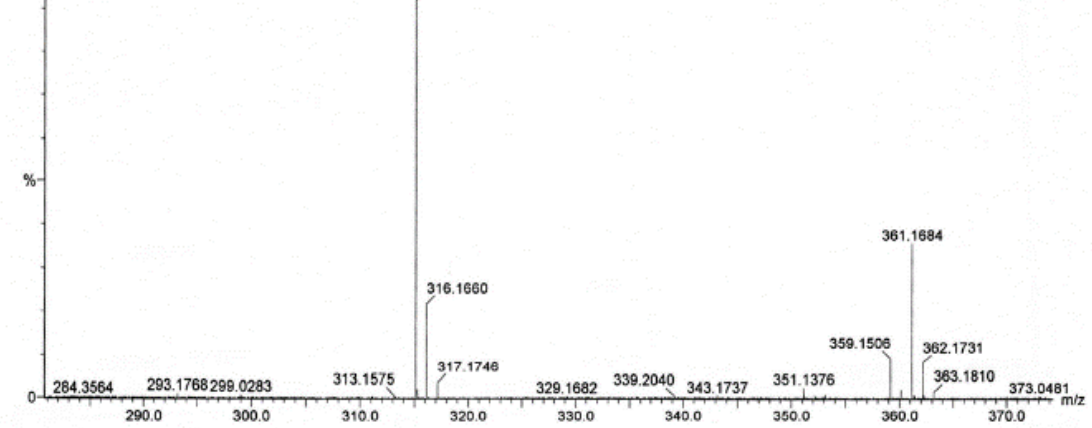

$\begin{array}{llll}\text { Minimum: } & & -1.5 \\ \text { Maximum: } & 5.0 & 3.0 & 50.0\end{array}$

Mass Calc, Mass mDa PPM

i-FIT i-FIT (Norm) Formula

$\begin{array}{llllllllll}315.1596 & 315.1596 & 0.0 & 0.0 & 8.5 & 109.2 & 0.0 & \text { C19 } & \text { H23 } & \text { O4 }\end{array}$ 
Figure S166. IR spectrum of fortalpinoid N (14).

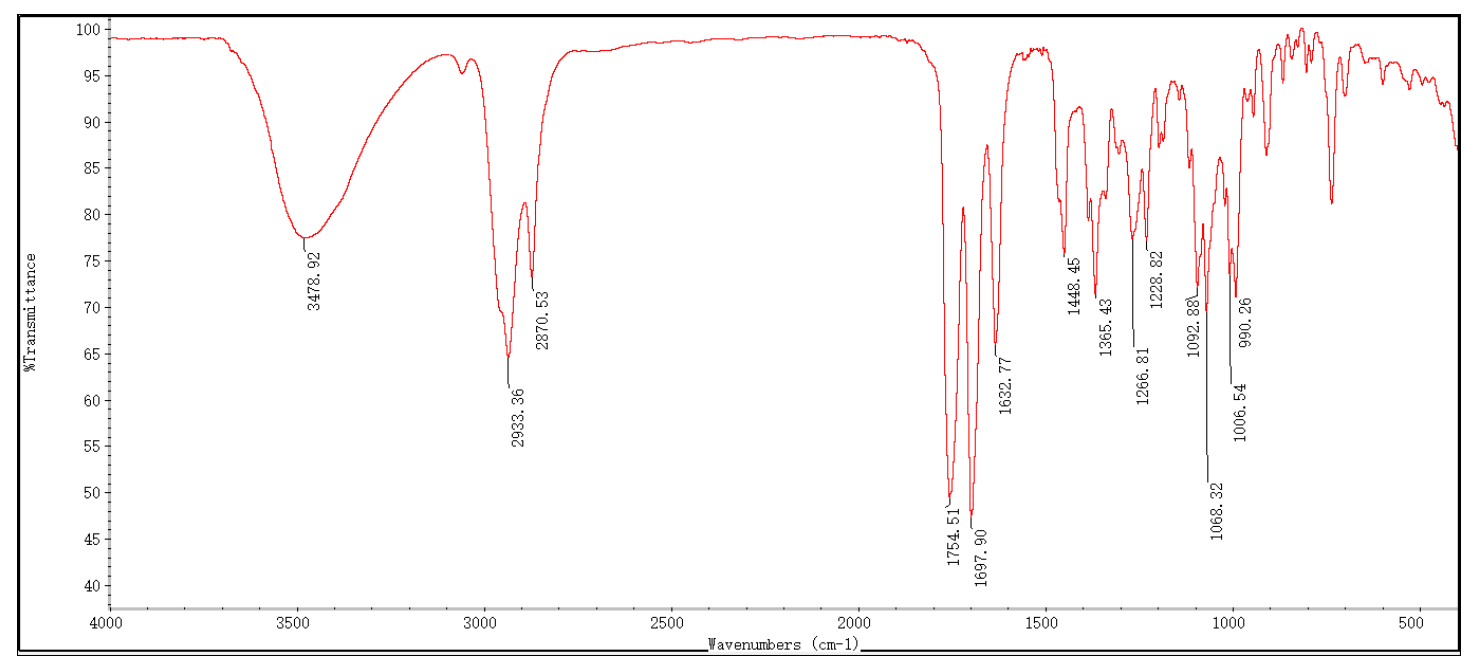

Figure S167. ${ }^{1} \mathrm{H}$ NMR spectrum of fortalpinoid O (15) in methanol- $d_{4}$.

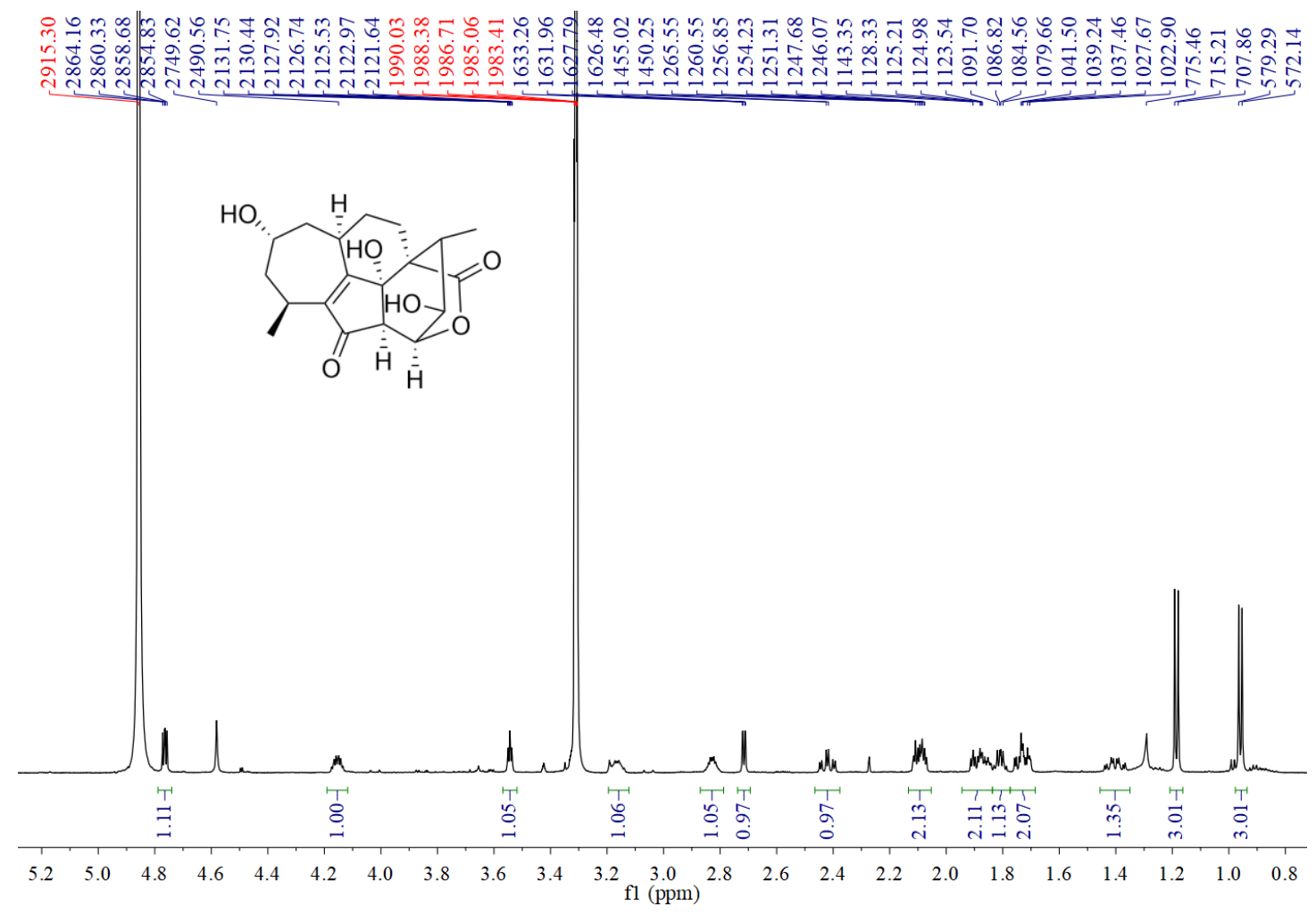


Figure S168. ${ }^{13} \mathrm{C}$ NMR spectrum of fortalpinoid O (15) in methanol- $d_{4}$.
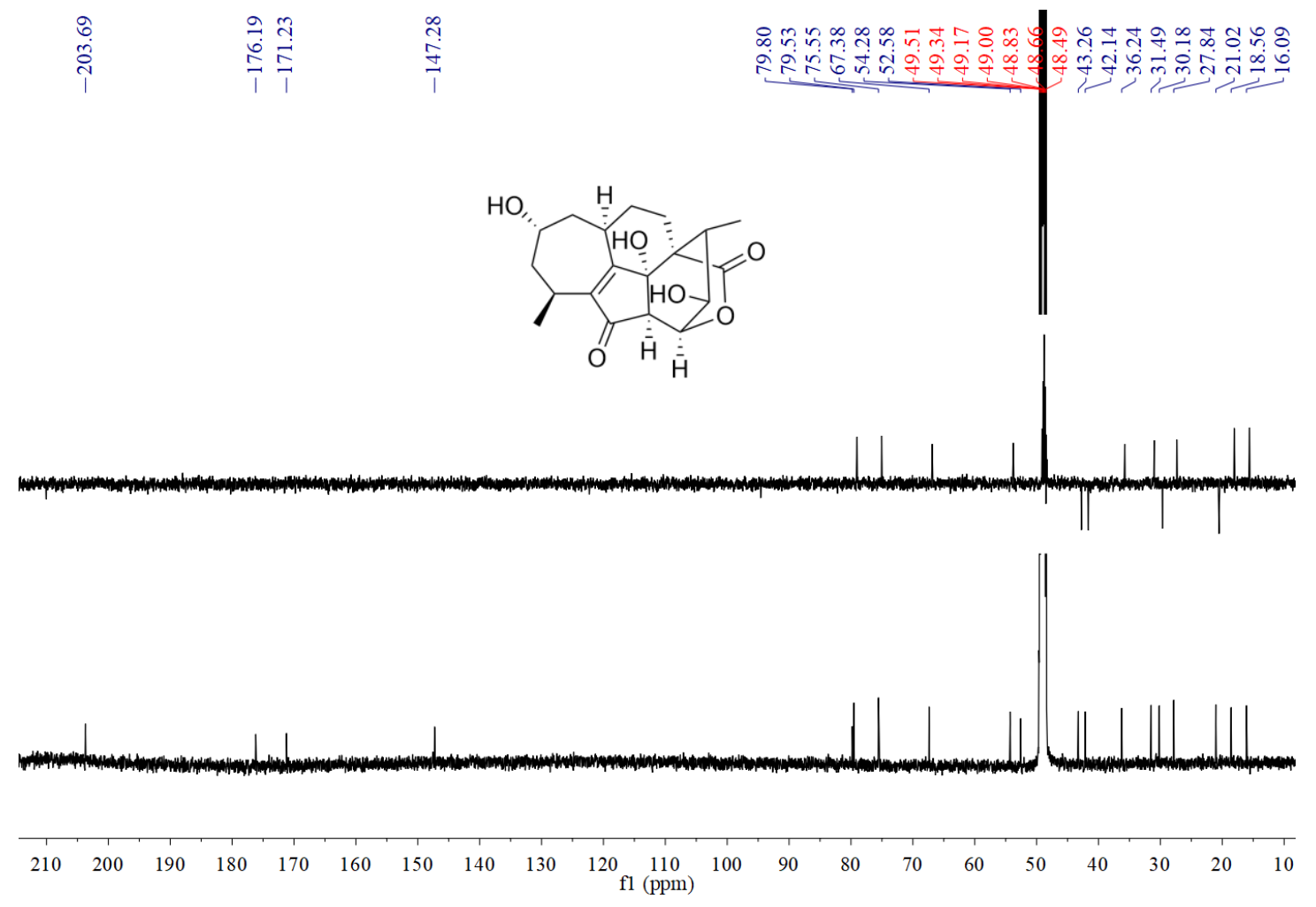

Figure S169. HSQC spectrum of fortalpinoid O (15) in methanol- $d_{4}$.

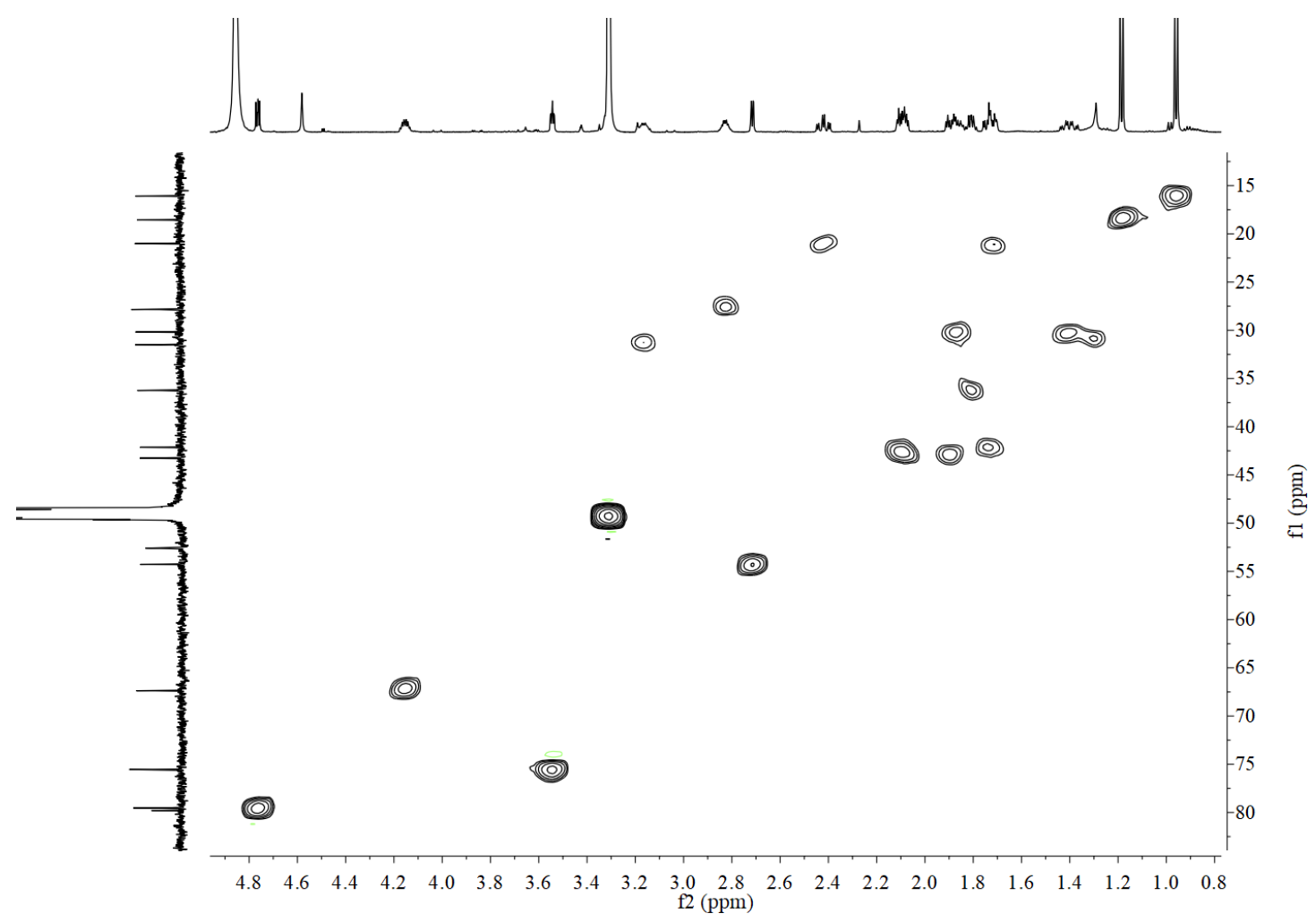


Figure S170. HMBC spectrum of fortalpinoid O (15) in methanol- $d_{4}$.

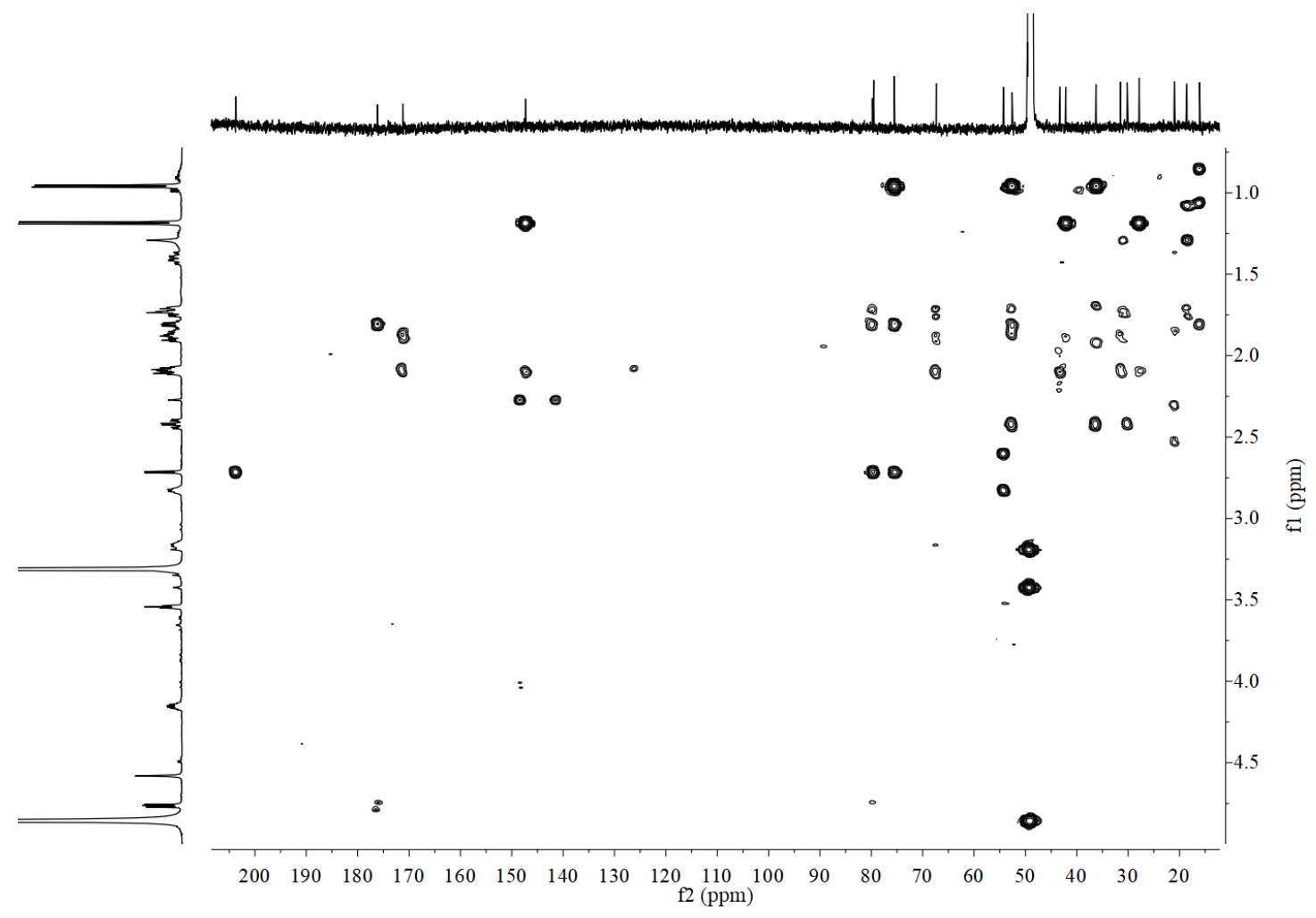

Figure S171. ${ }^{1} \mathrm{H}-{ }^{1} \mathrm{H}$ COSY spectrum of fortalpinoid O (15) in methanol- $d_{4}$.

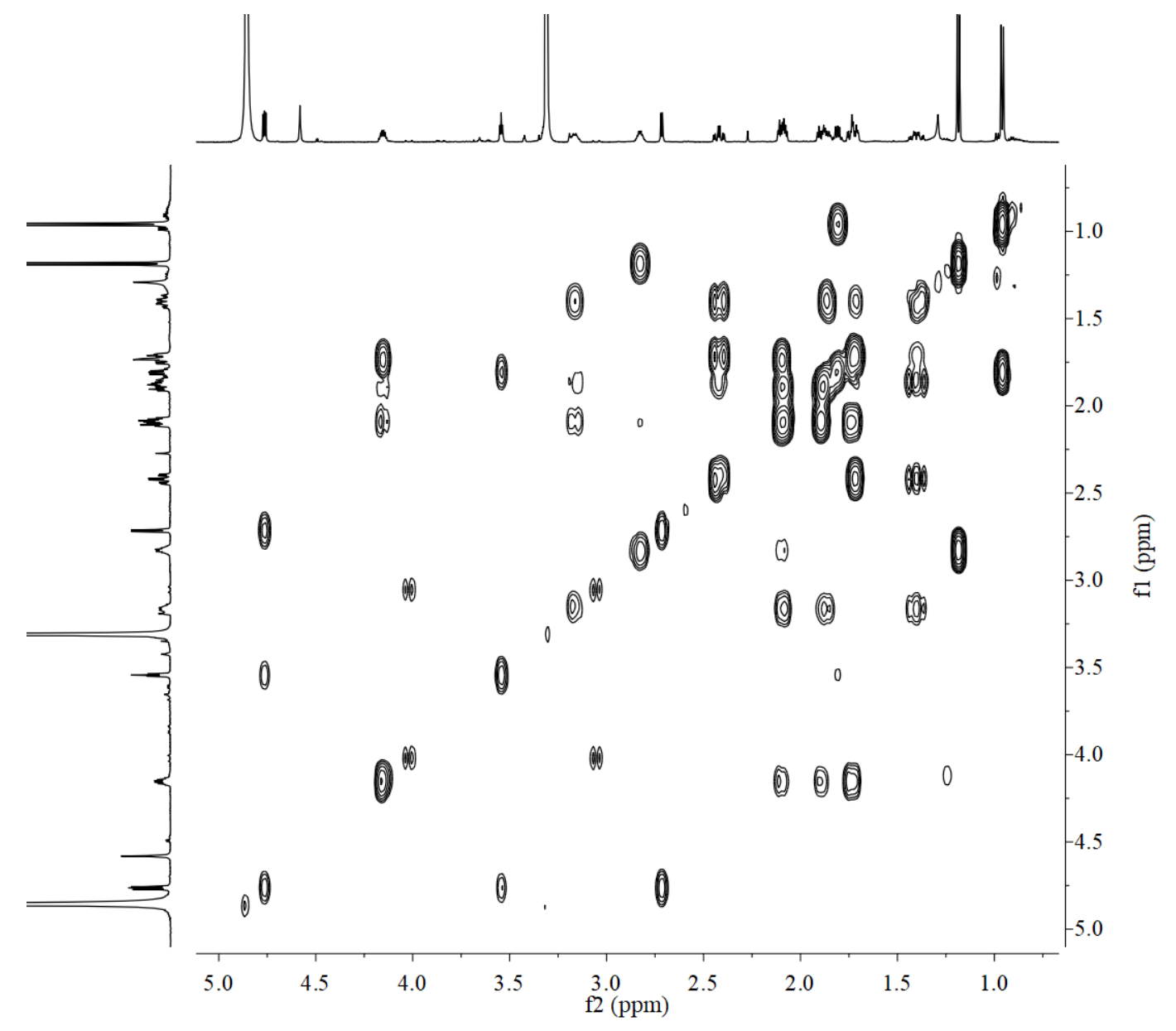


Figure S172. NOESY spectrum of fortalpinoid O (15) in methanol- $d_{4}$.

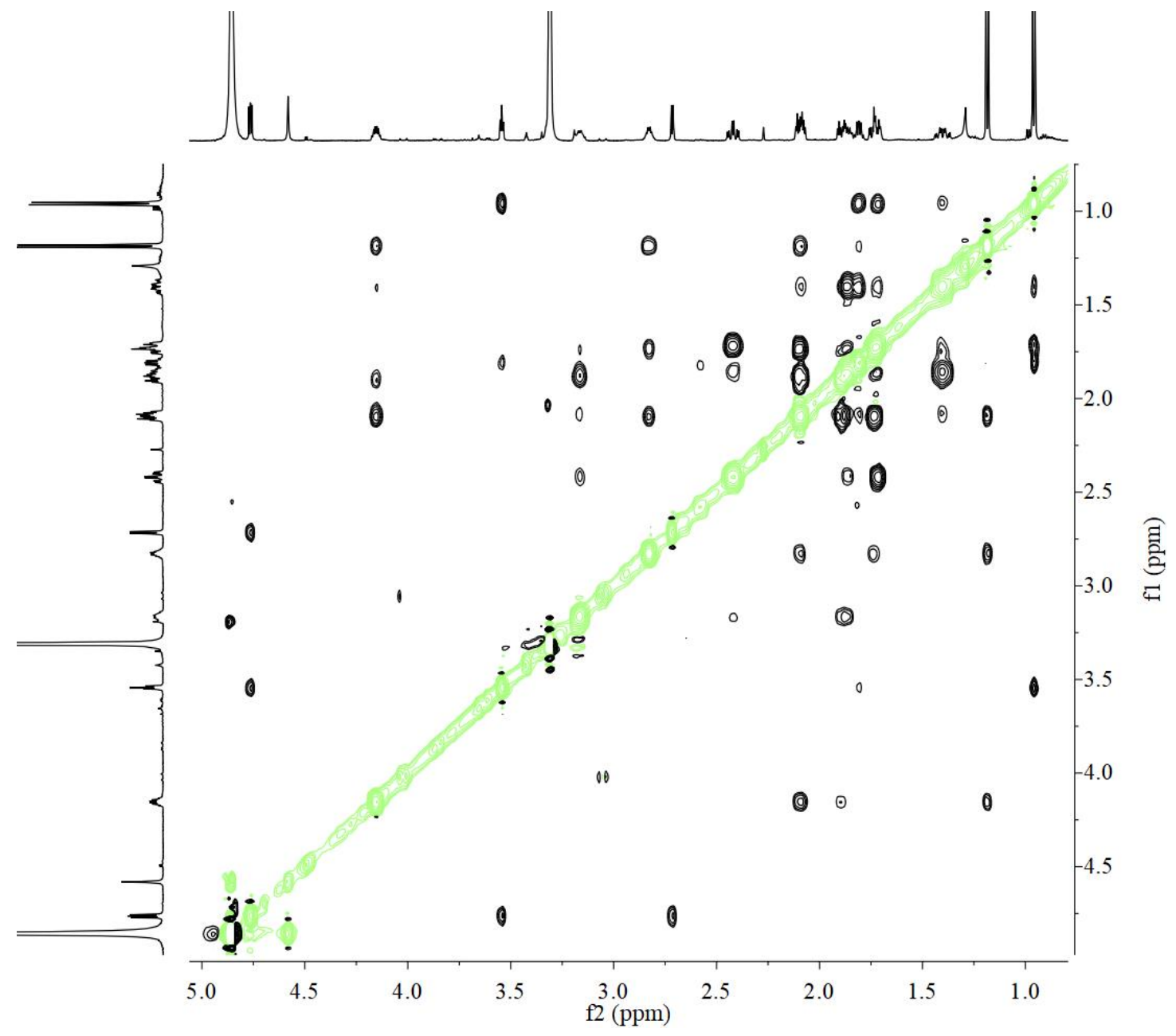

Figure S173. (-)-ESIMS spectrum of fortalpinoid O (15).

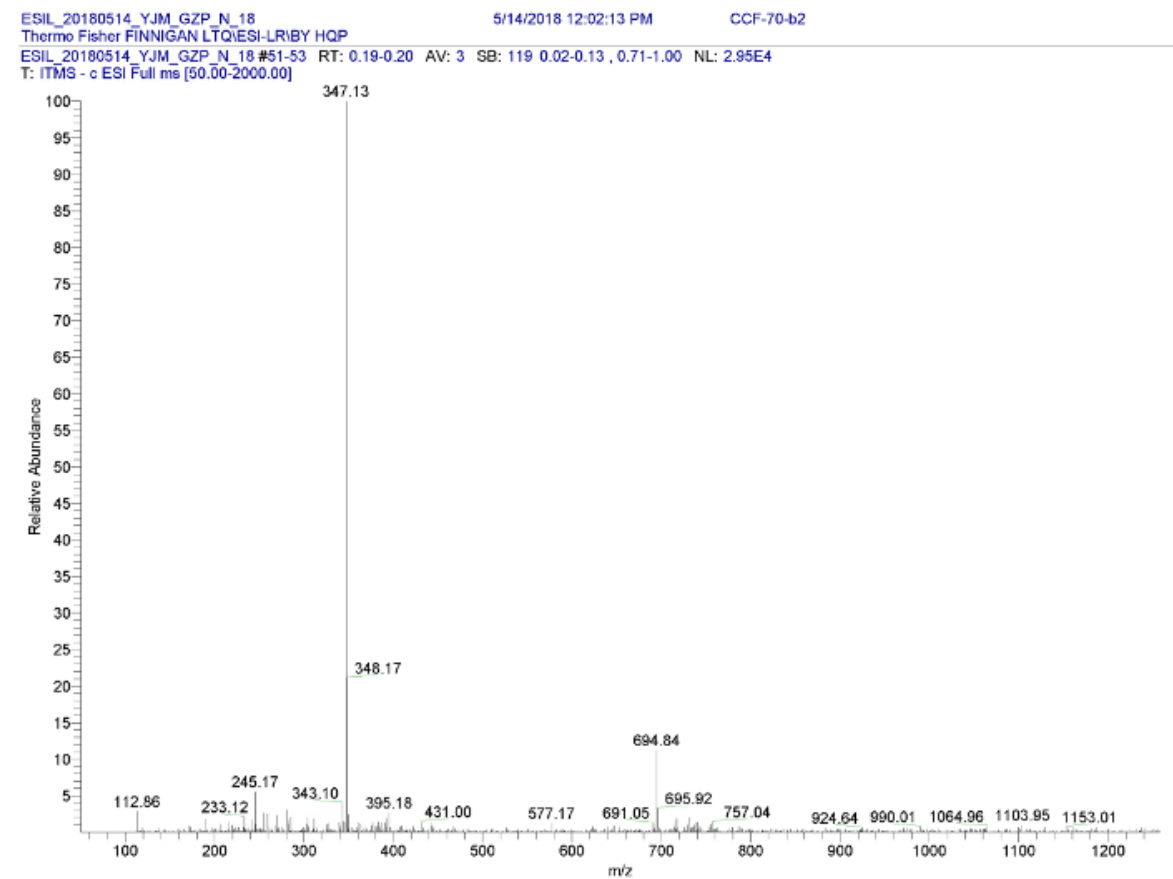


Figure S174. (-)-HRESIMS spectrum of fortalpinoid O (15).

Qualitative Analysis Report

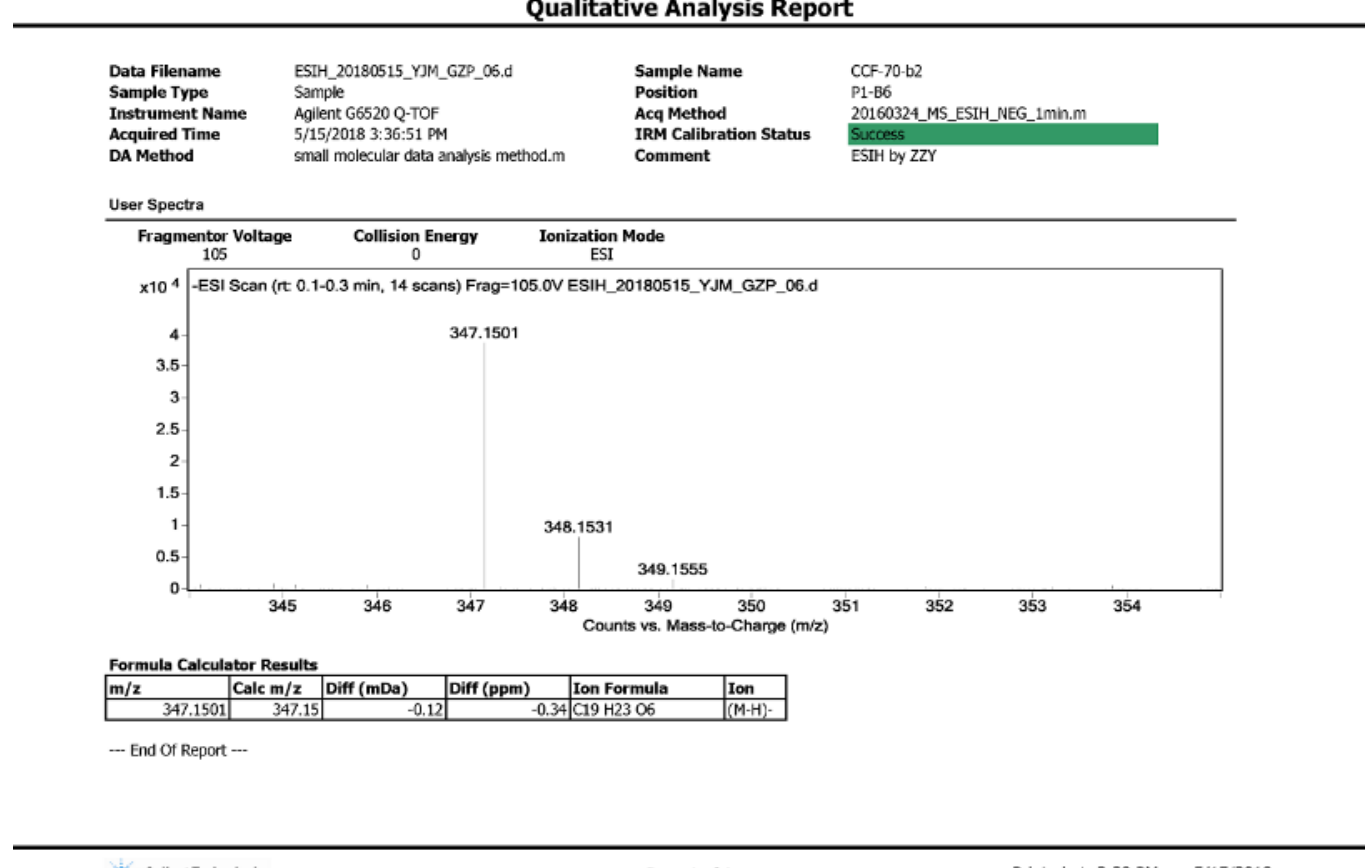

Aglitent Technologies $\quad$ Page 1 of 1

Printed at: 3:39 PM on: 5/15/2018

Figure S175. IR spectrum of fortalpinoid O (15).

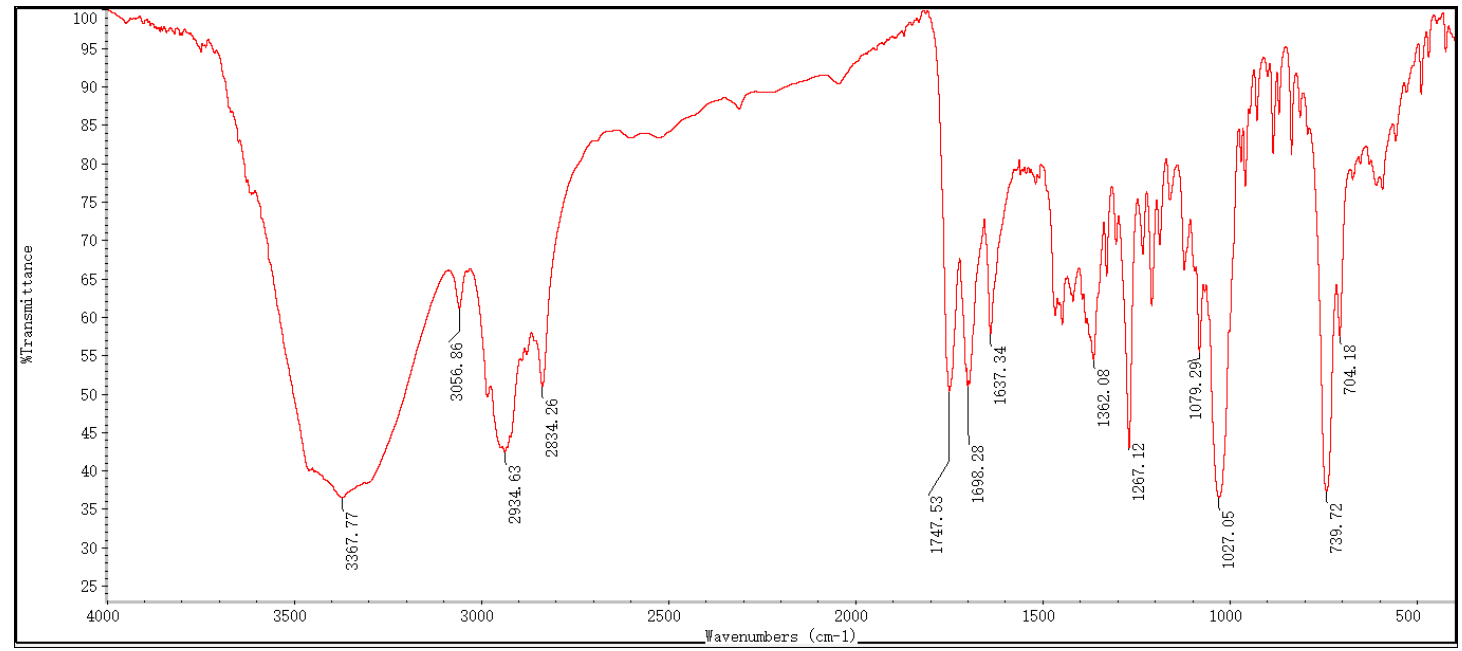


Figure S176. ${ }^{1} \mathrm{H}$ NMR spectrum of fortalpinoid $\mathrm{P}(\mathbf{1 6})$ in $\mathrm{CDCl}_{3}$.
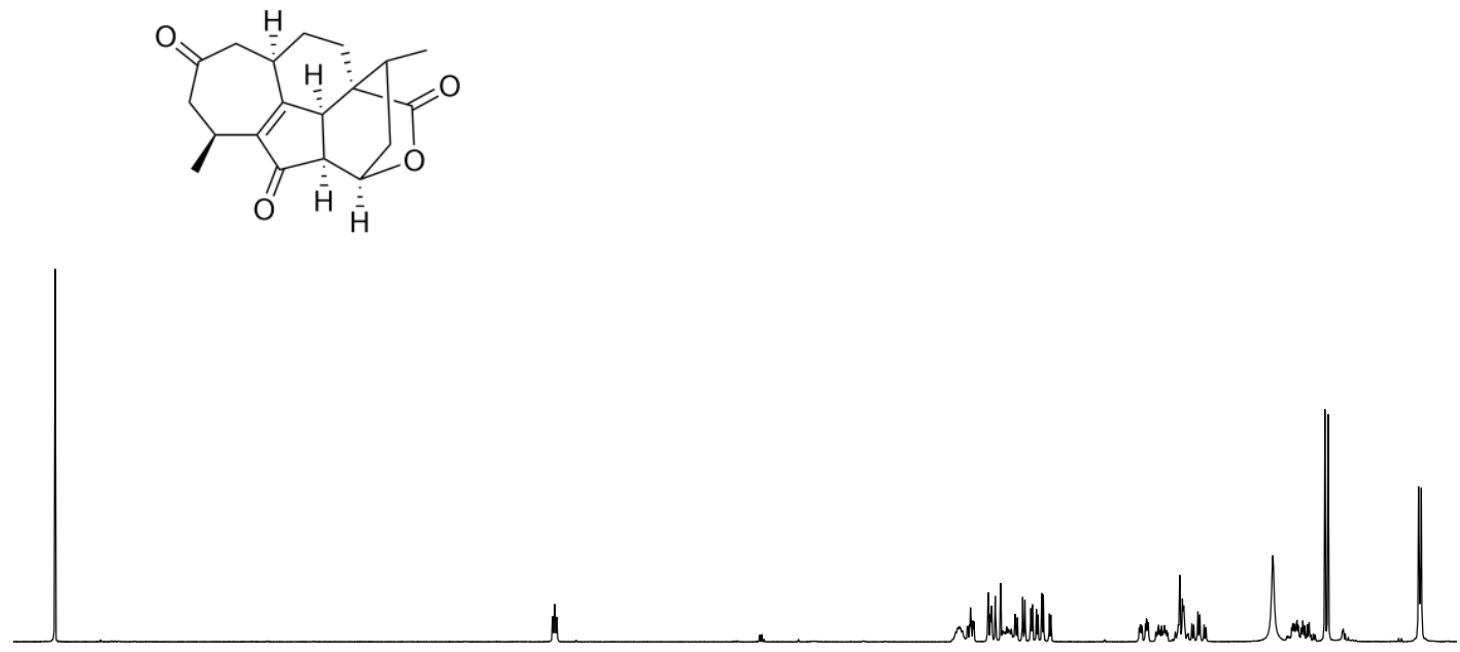

$\begin{array}{llllllllllllllllllllllllllllllllll}7.4 & 7.2 & 7.0 & 6.8 & 6.6 & 6.4 & 6.2 & 6.0 & 5.8 & 5.6 & 5.4 & 5.2 & 5.0 & 4.8 & 4.6 & 4.4 & 4.2 & 4.0 & 3.8 & 3.6 & 3.4 & 3.2 & 3.0 & 2.8 & 2.6 & 2.4 & 2.2 & 2.0 & 1.8 & 1.6 & 1.4 & 1.2 & 1.0 & 0.8\end{array}$ fl $(\mathrm{ppm})$

Figure S177. ${ }^{13} \mathrm{C}$ NMR spectrum of fortalpinoid $\mathrm{P}(\mathbf{1 6})$ in $\mathrm{CDCl}_{3}$.
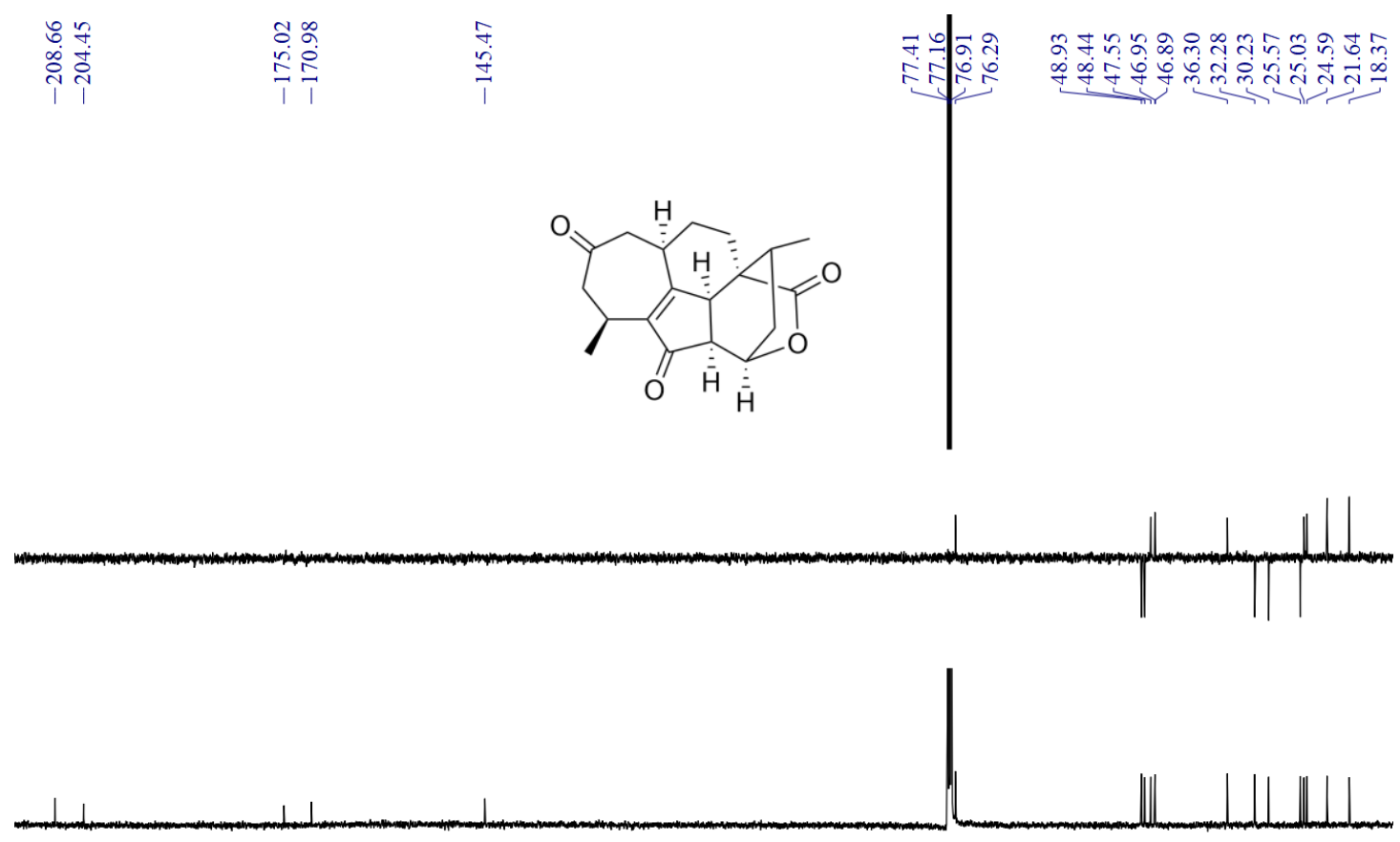

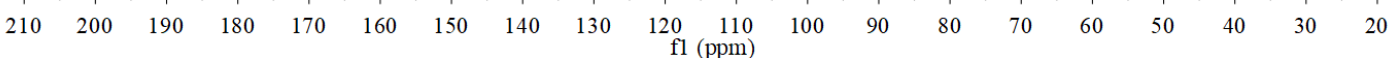


Figure S178. HSQC spectrum of fortalpinoid $\mathrm{P}(\mathbf{1 6})$ in $\mathrm{CDCl}_{3}$.

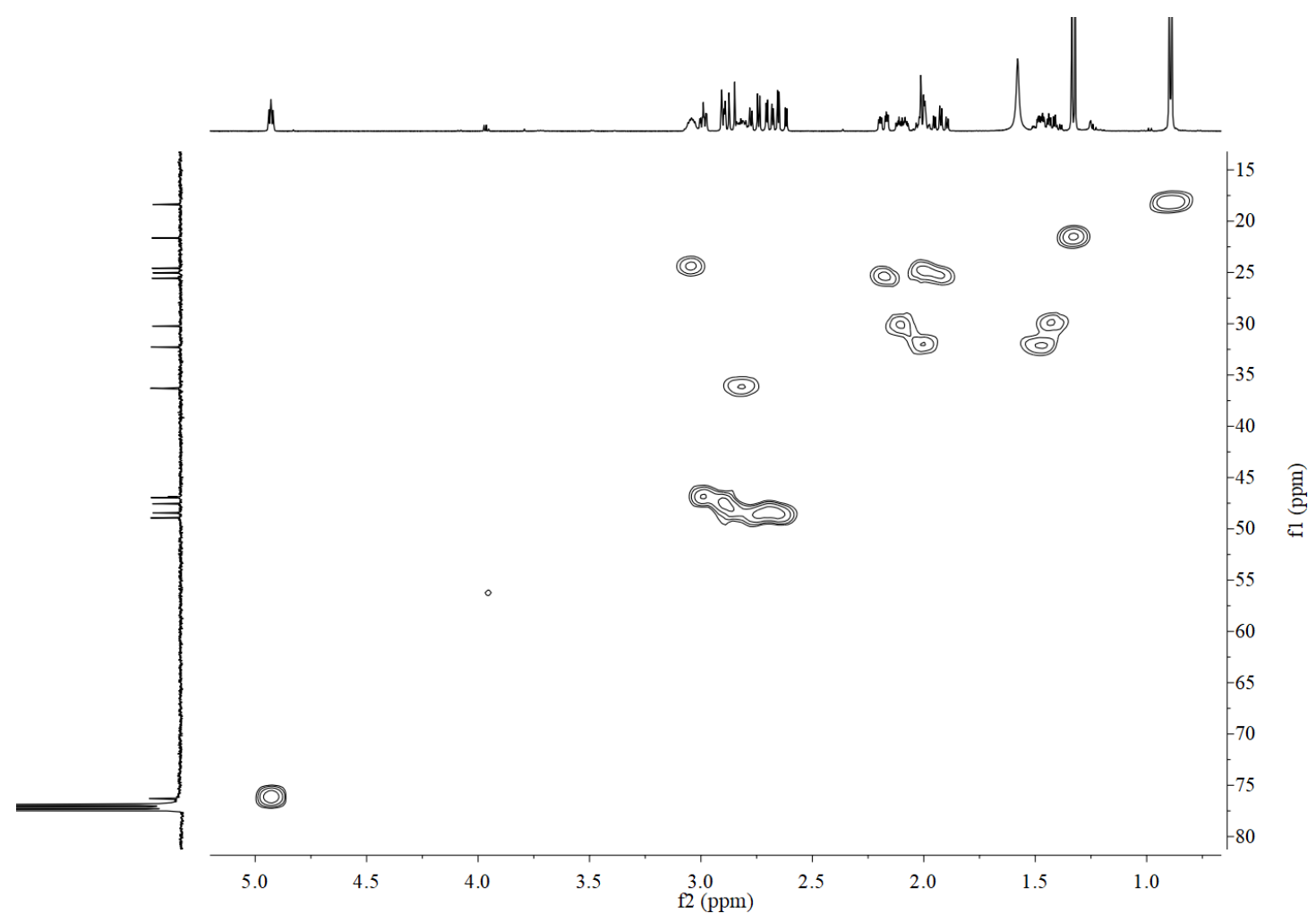

Figure S179. HMBC spectrum of fortalpinoid $\mathrm{P}(\mathbf{1 6})$ in $\mathrm{CDCl}_{3}$.

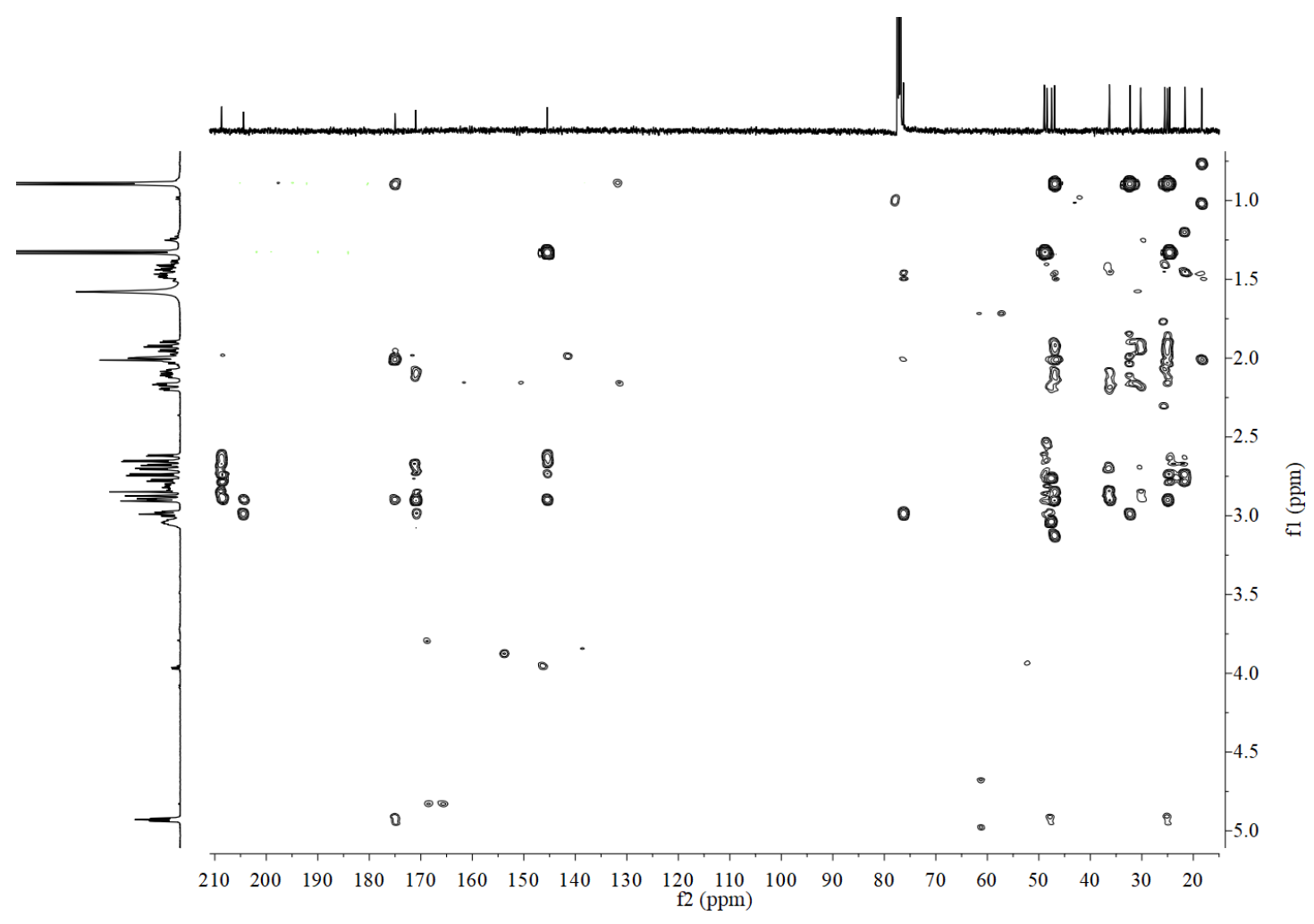


Figure S180. ${ }^{1} \mathrm{H}-{ }^{1} \mathrm{H}$ COSY spectrum of fortalpinoid $\mathrm{P}(\mathbf{1 6})$ in $\mathrm{CDCl}_{3}$.

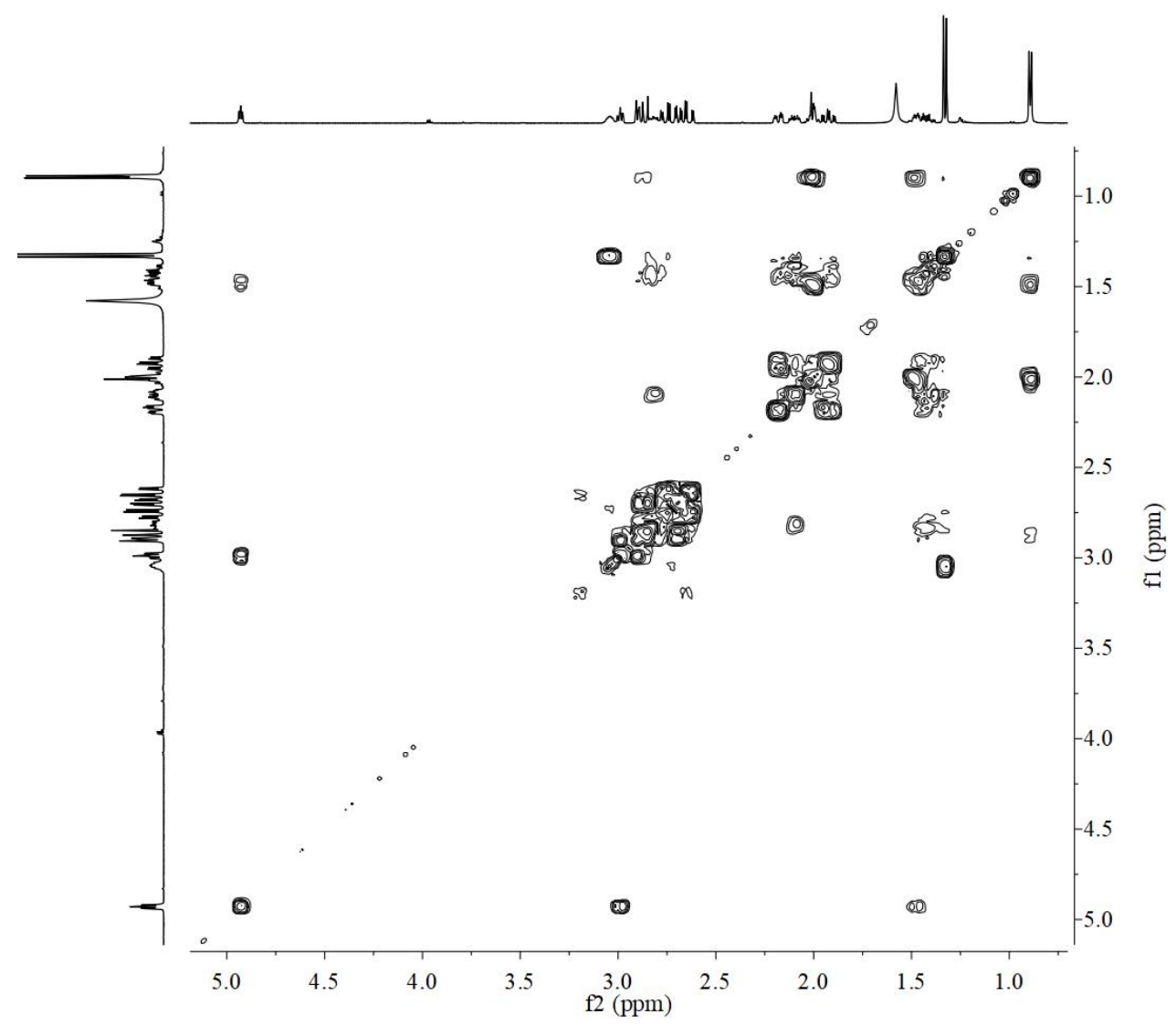

Figure S181. NOESY spectrum of fortalpinoid $\mathrm{P}(\mathbf{1 6})$ in $\mathrm{CDCl}_{3}$.

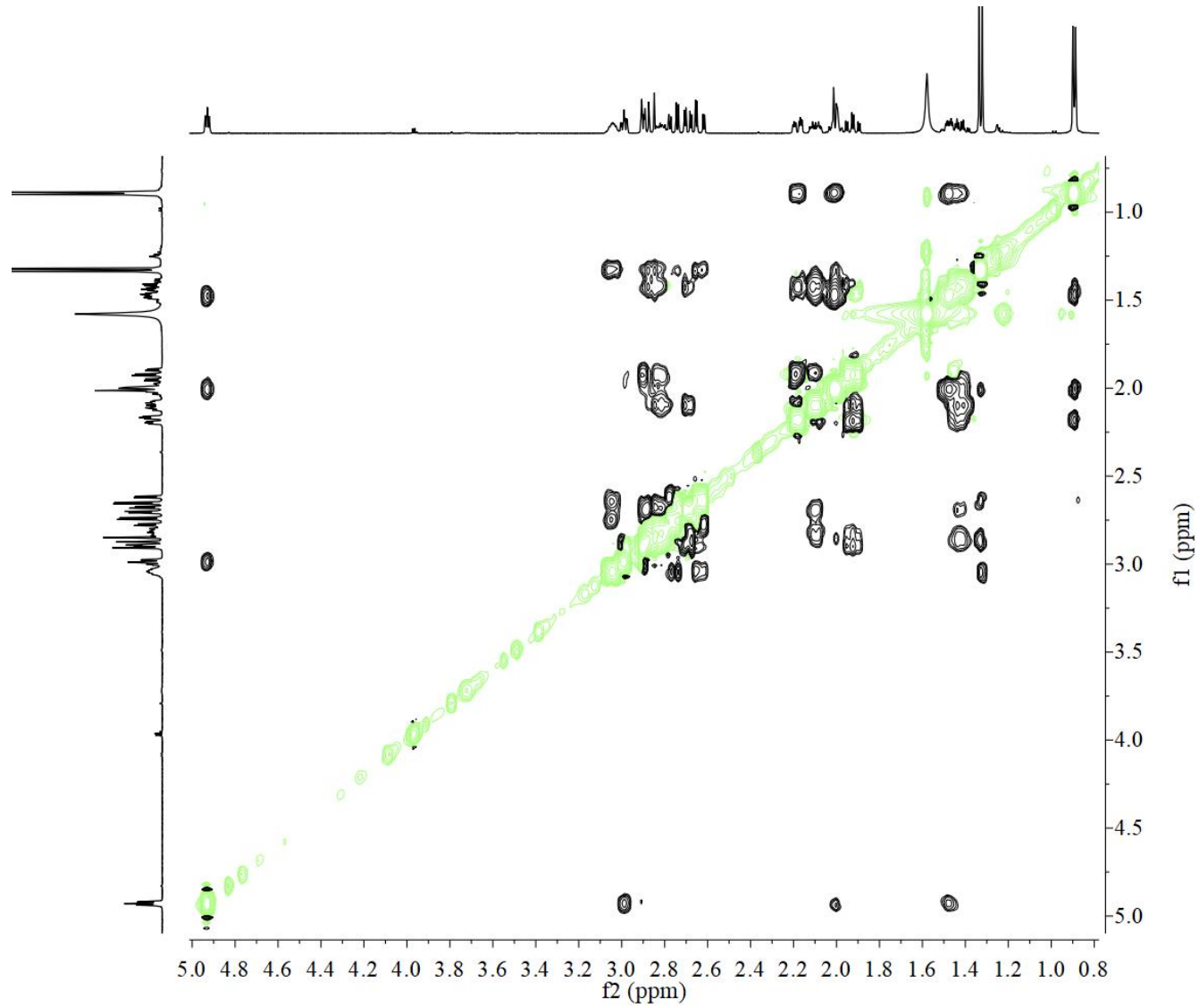


Figure S182. (-)-ESIMS spectrum of fortalpinoid P (16).

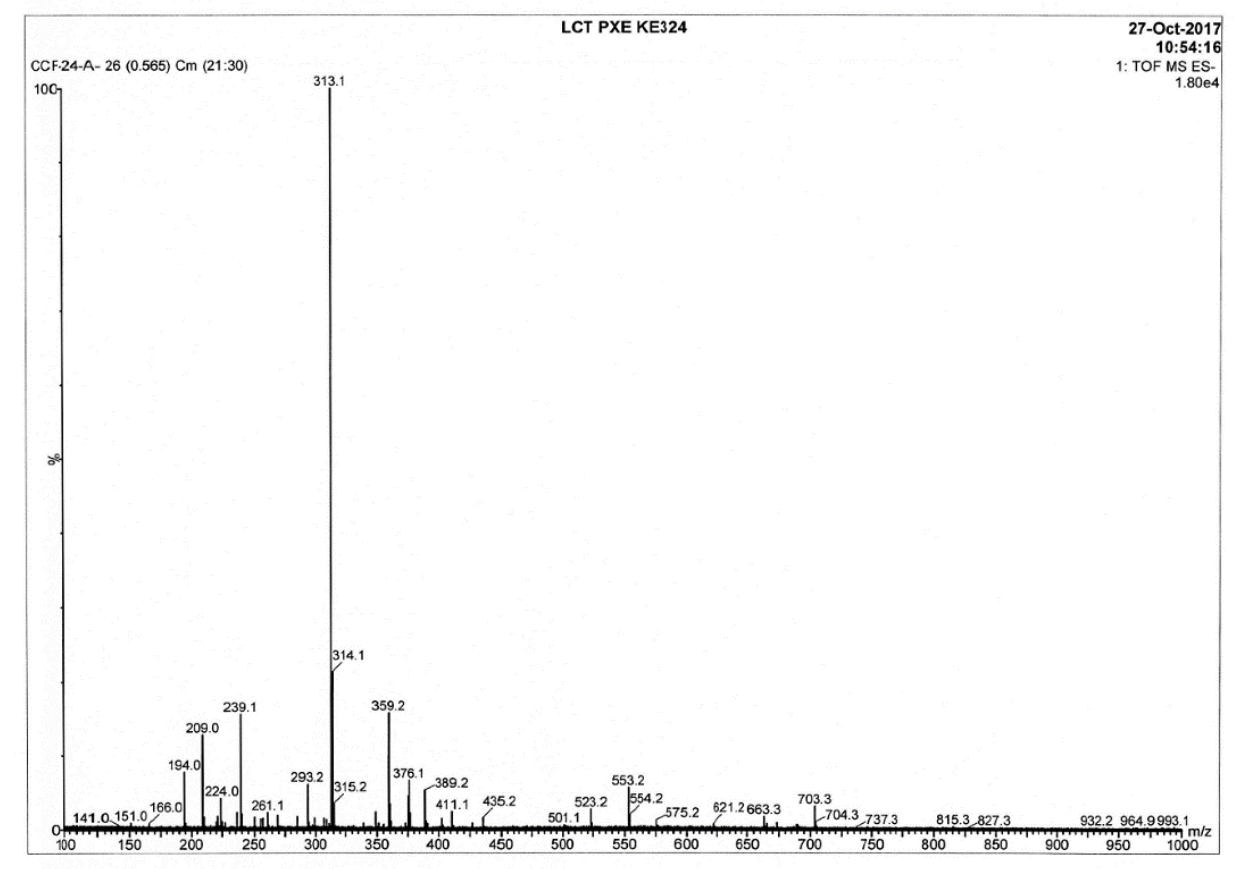

Figure S183. (-)-HRESIMS spectrum of fortalpinoid P (16).

Elemental Composition Report

Page 1

Single Mass Analysis

Tolerance $=2.0$ PPM I DBE: $\min =-1.5, \max =50.0$

Element prediction: Of

Number of isotope peaks used for $\mathrm{i}-\mathrm{FIT}=3$

Monoisotopic Mass, Even Electron lons

150 formula(e) evaluated with 1 results within limits (up to 50 best isotopic matches for each mass)

$\begin{array}{llll}\text { C: } 0-500 & \text { H: } 0-1000 & \text { O: } 0-500 \quad \mathrm{Na}: 0-2\end{array}$

LCT PXE KE 324

27-0ct-2017

$10: 54: 16$
CCF-24-A- $10(0.212) \mathrm{Cm}(8: 11)$

1: TOF MS ES-

CCF-24-A- $10(0.212) \mathrm{Cm}(8.11)$
$7.20 \mathrm{e}+003$

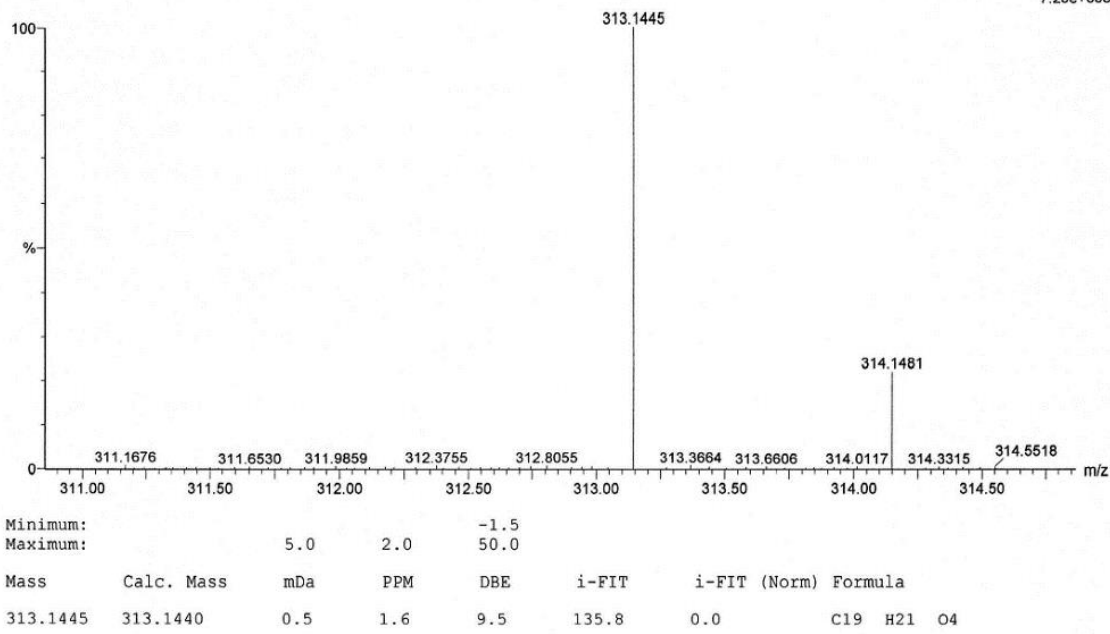


Figure S184. IR spectrum of fortalpinoid P (16).

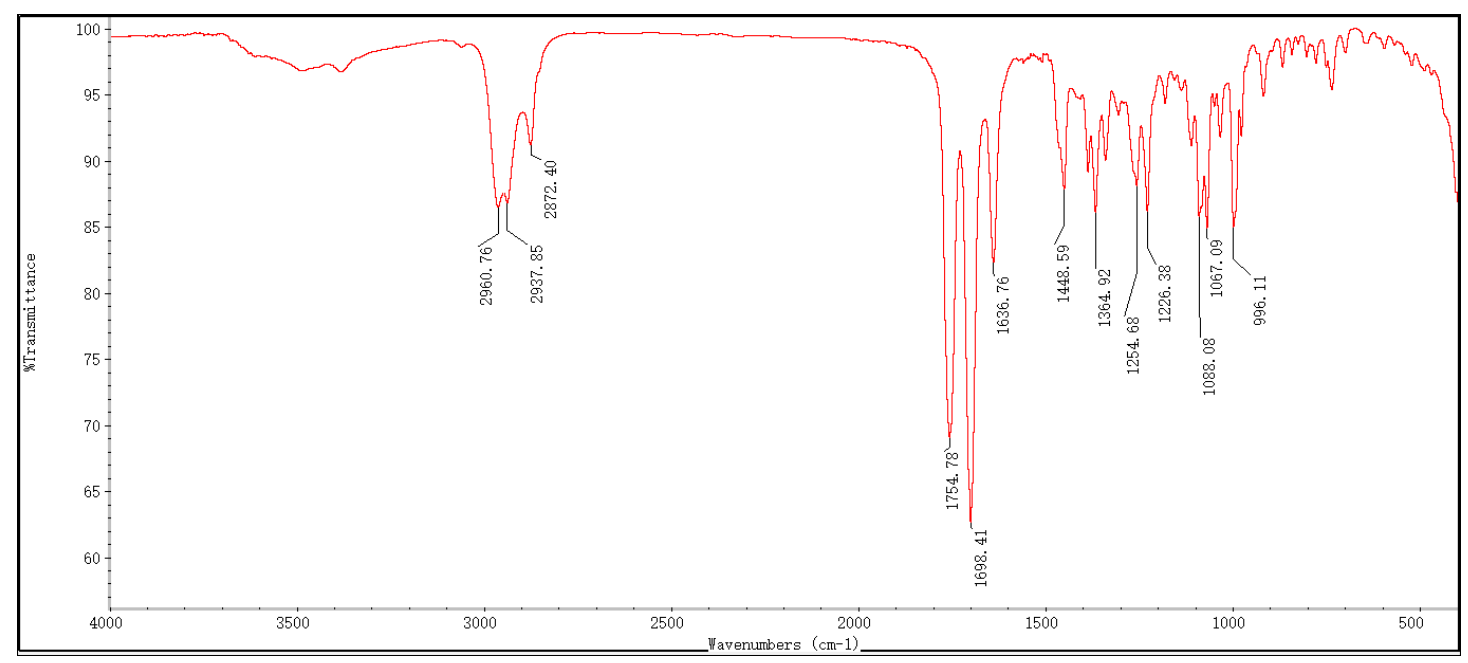

Figure S185. ${ }^{1} \mathrm{H}$ NMR spectrum of fortalpinoid Q (17) in $\mathrm{CDCl}_{3}$.

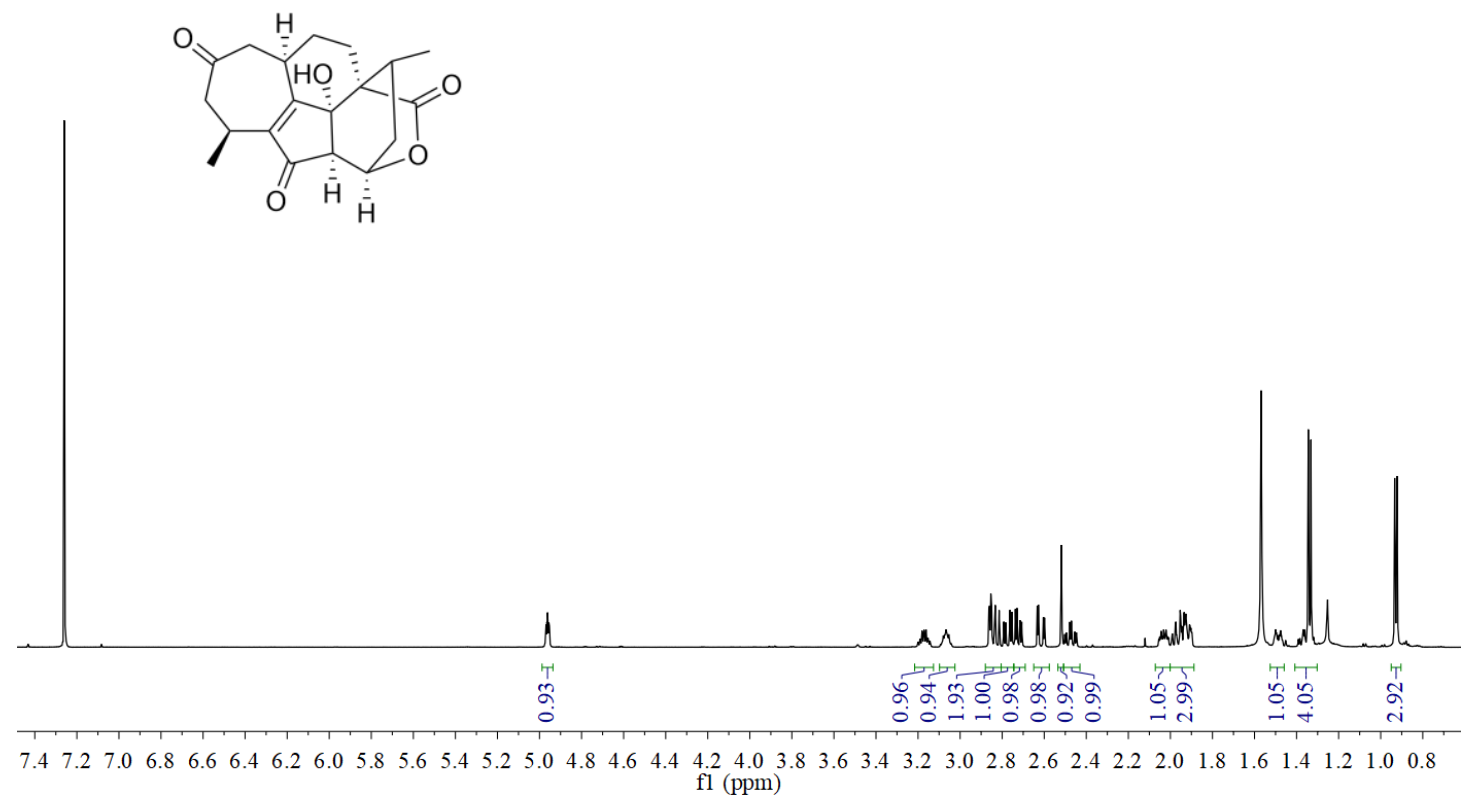


Figure S186. ${ }^{13} \mathrm{C}$ NMR spectrum of fortalpinoid Q (17) in $\mathrm{CDCl}_{3}$.
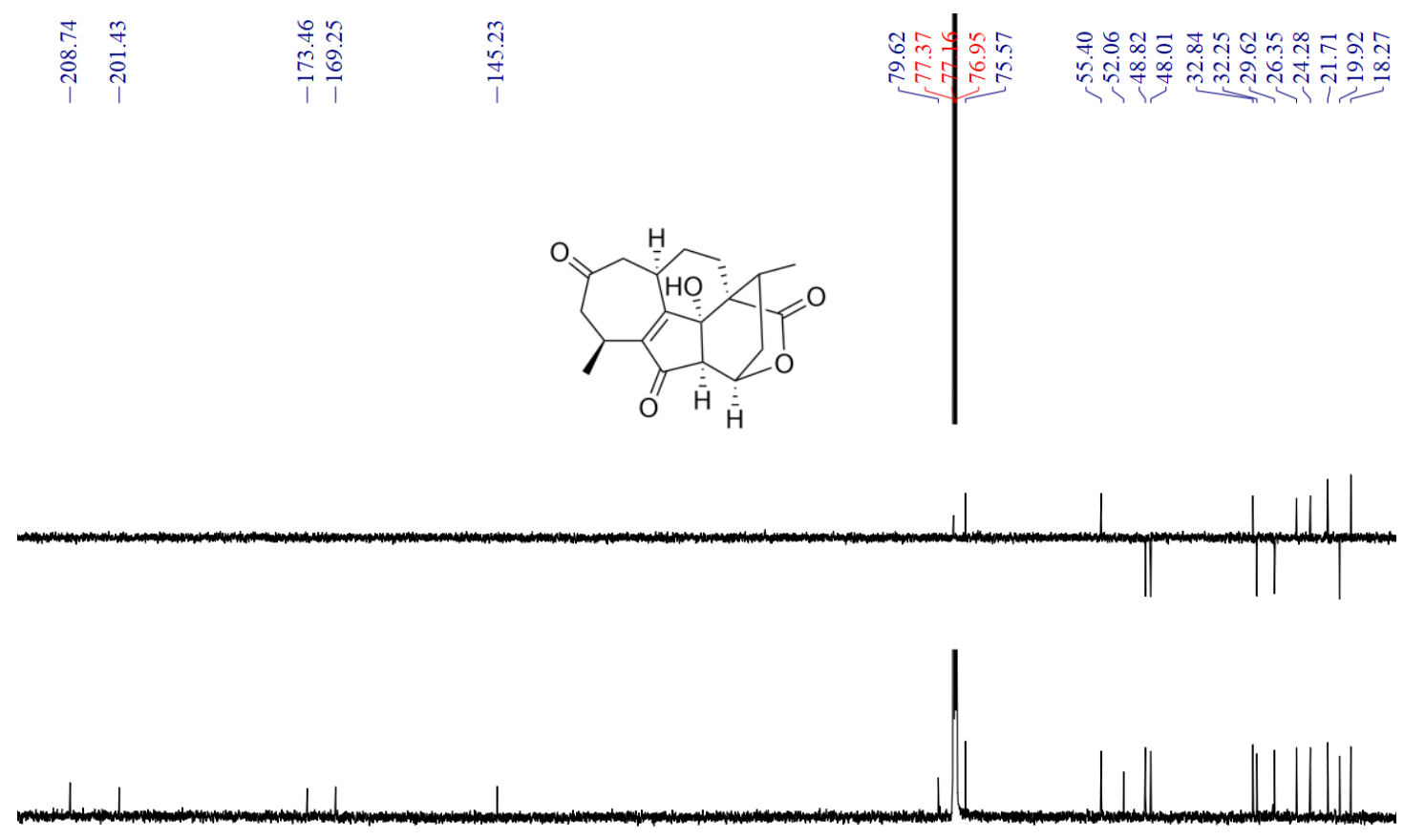

$\begin{array}{rrrrrrrrrrrrrrrrrrrr}210 & 200 & 190 & 180 & 170 & 160 & 150 & 140 & 130 & \begin{array}{r}120 \\ \text { fl (ppm) }\end{array} & 100 & 90 & 80 & 70 & 60 & 50 & 40 & 30 & 20\end{array}$

Figure S187. HSQC spectrum of fortalpinoid Q (17) in $\mathrm{CDCl}_{3}$.

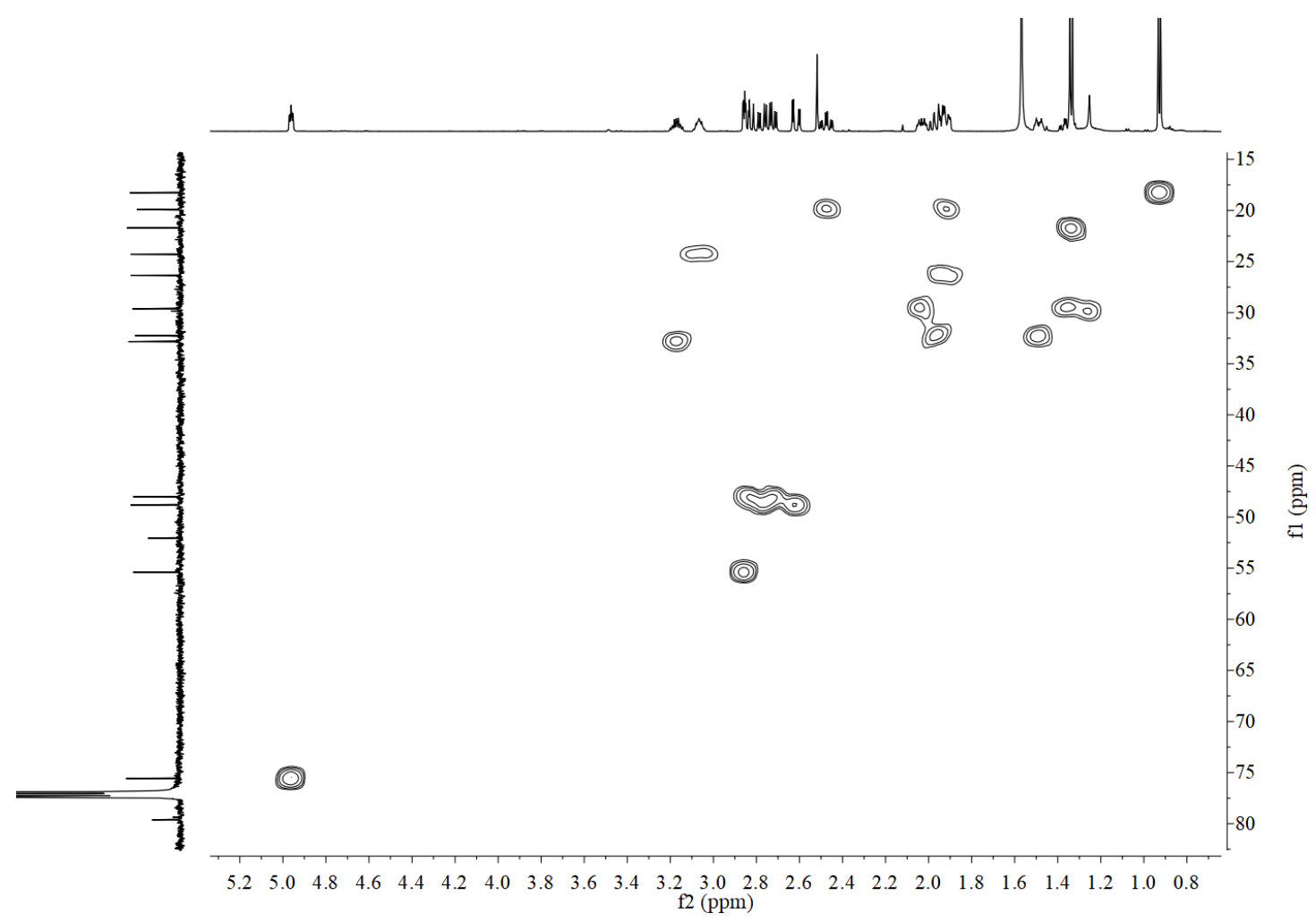


Figure S188. HMBC spectrum of fortalpinoid Q (17) in $\mathrm{CDCl}_{3}$.

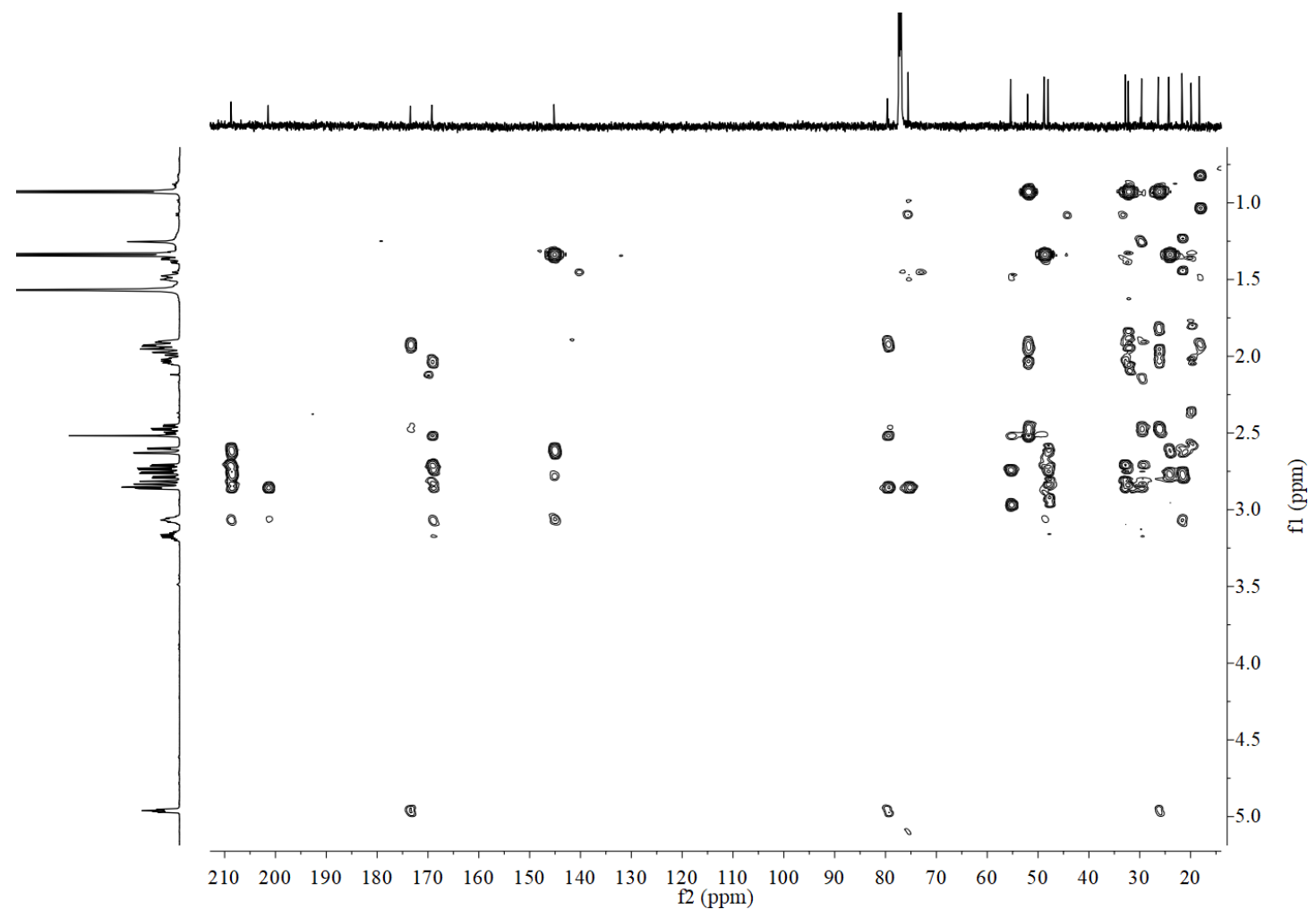

Figure S189. ${ }^{1} \mathrm{H}-{ }^{1} \mathrm{H}$ COSY spectrum of fortalpinoid Q (17) in $\mathrm{CDCl}_{3}$.

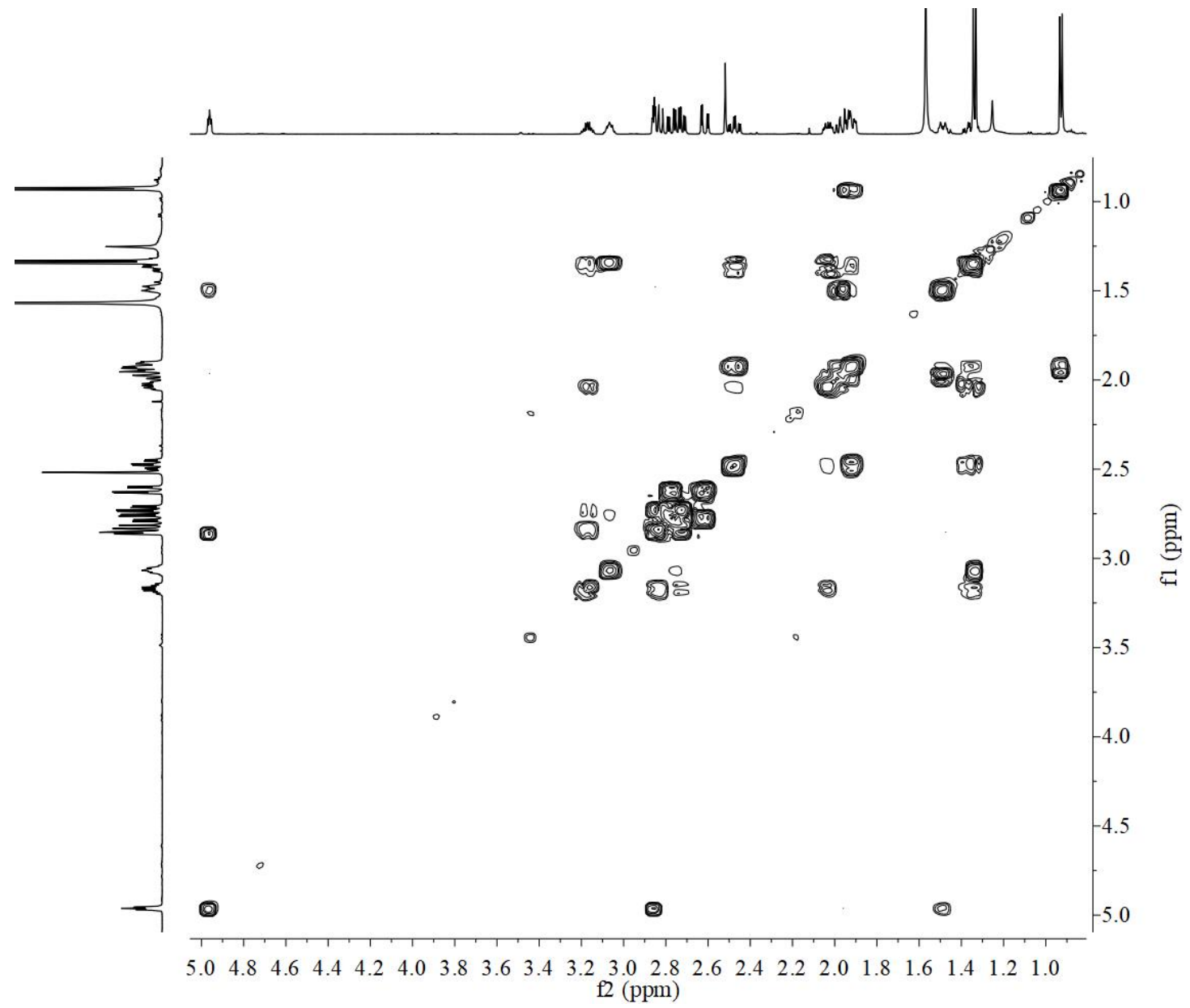


Figure S190. NOESY spectrum of fortalpinoid Q (17) in $\mathrm{CDCl}_{3}$.

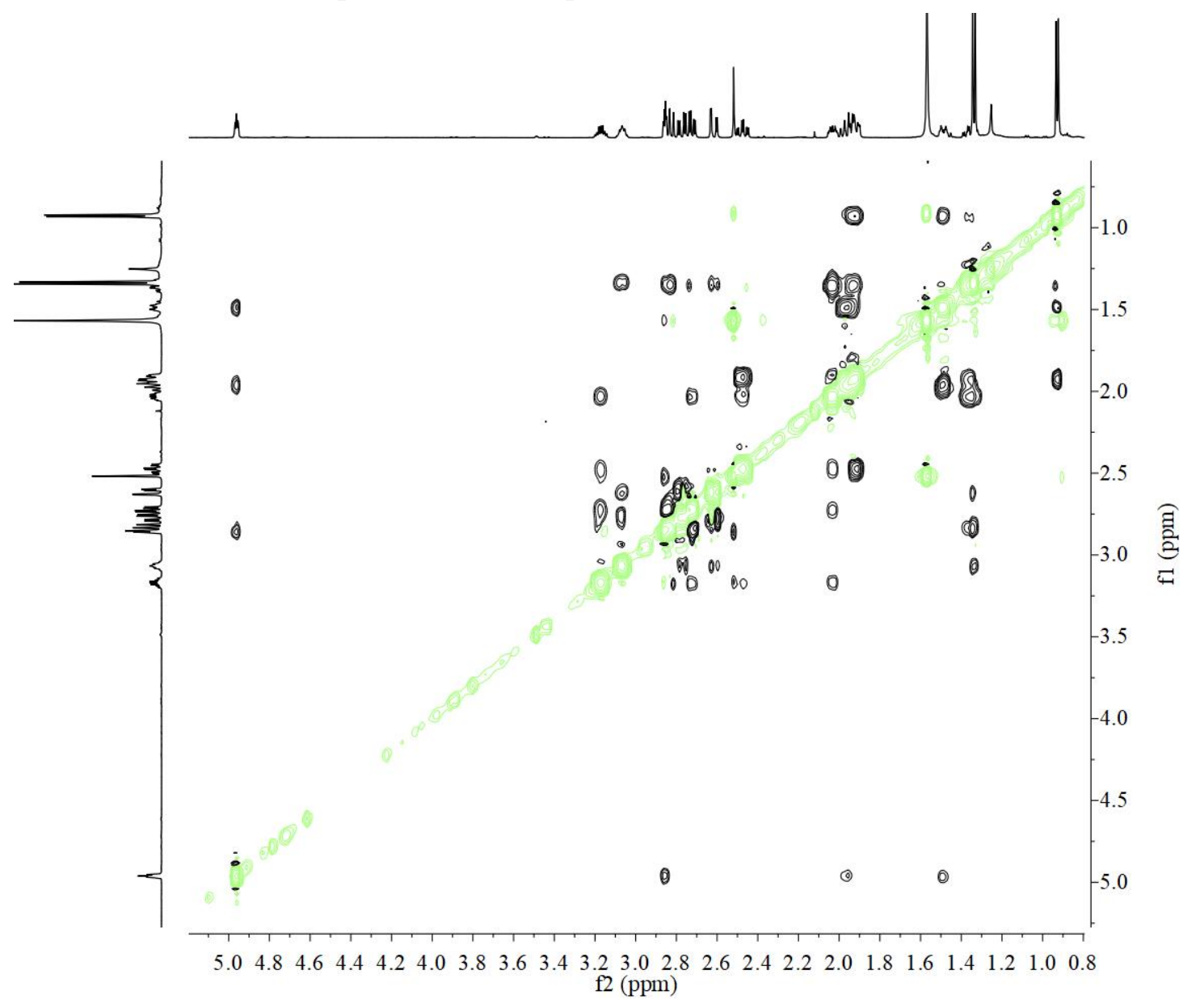

Figure S191. (+)-ESIMS spectrum of fortalpinoid Q (17).

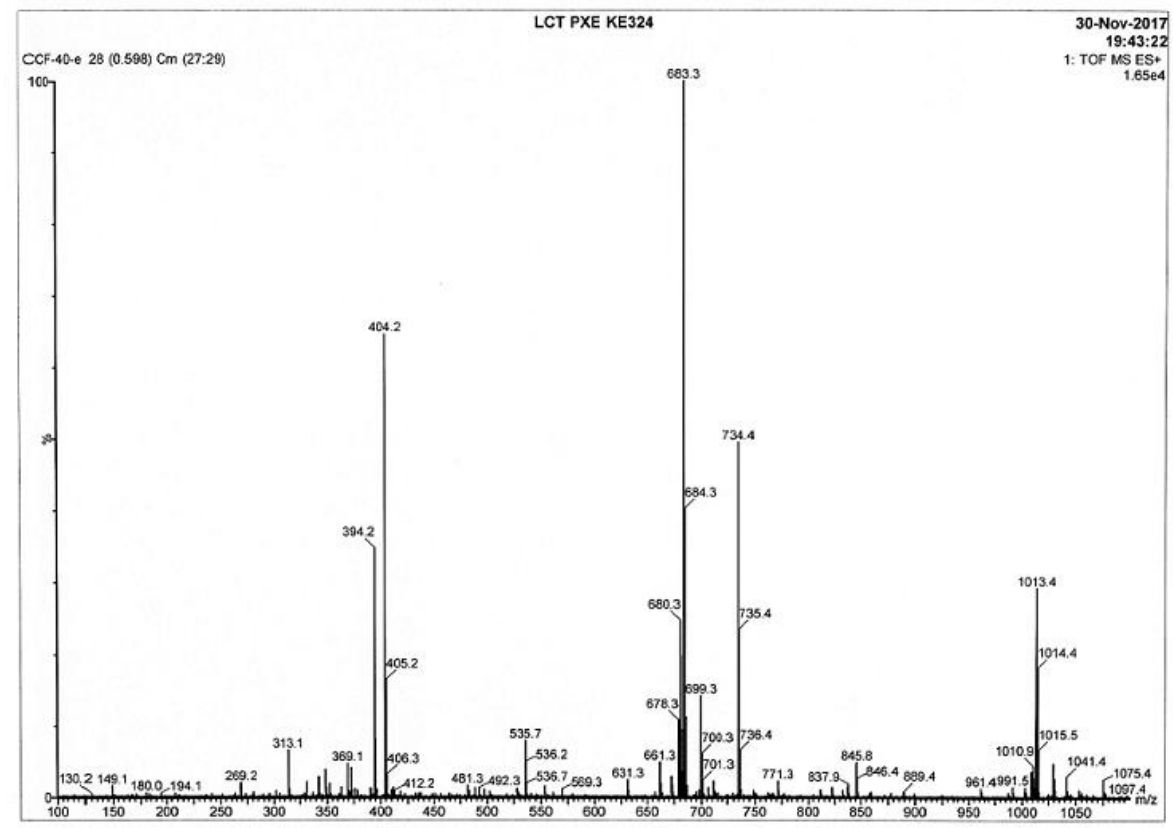


Figure S192. (-)-ESIMS spectrum of fortalpinoid Q (17).

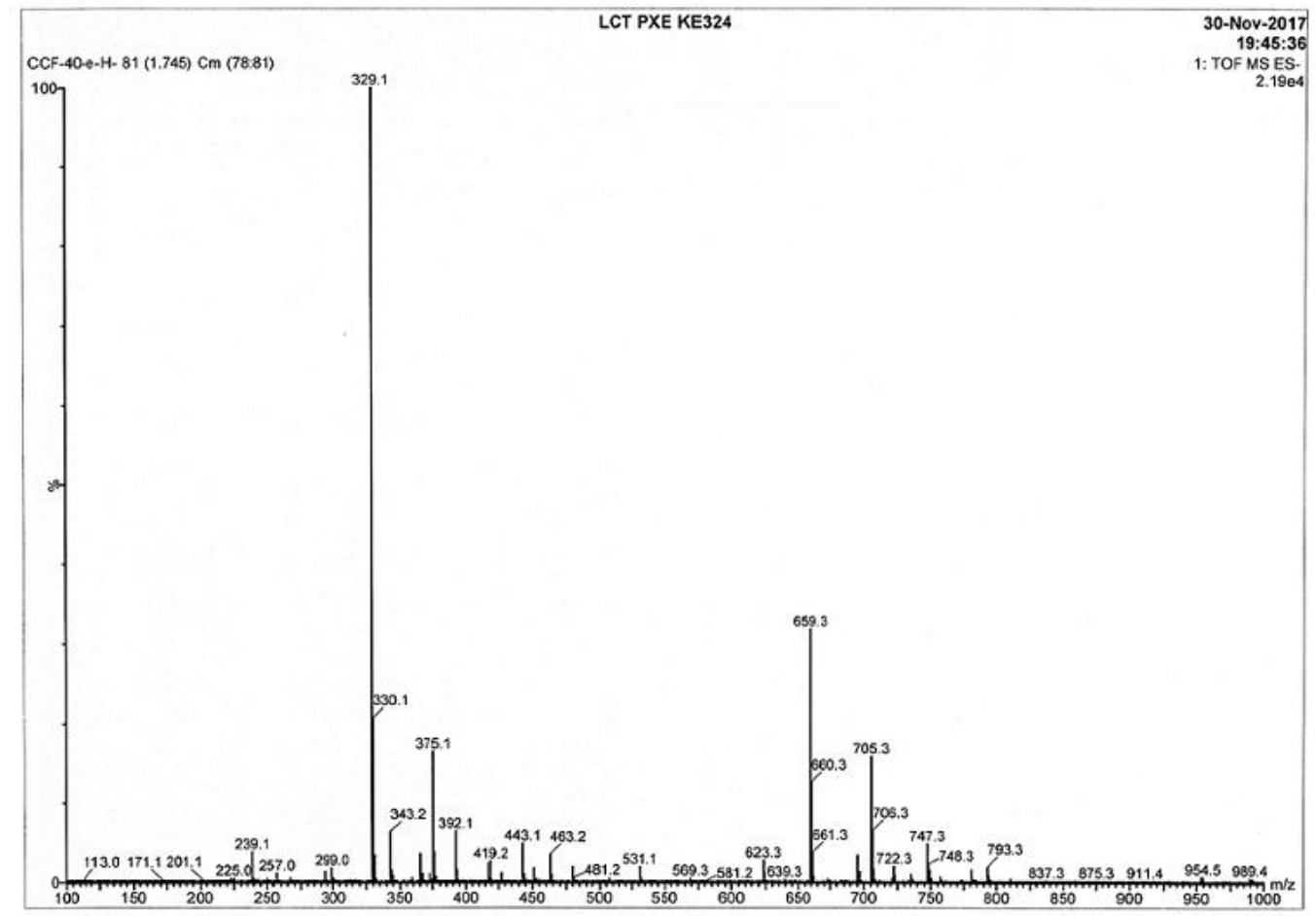

Figure S193. (-)-HRESIMS spectrum of fortalpinoid Q (17).

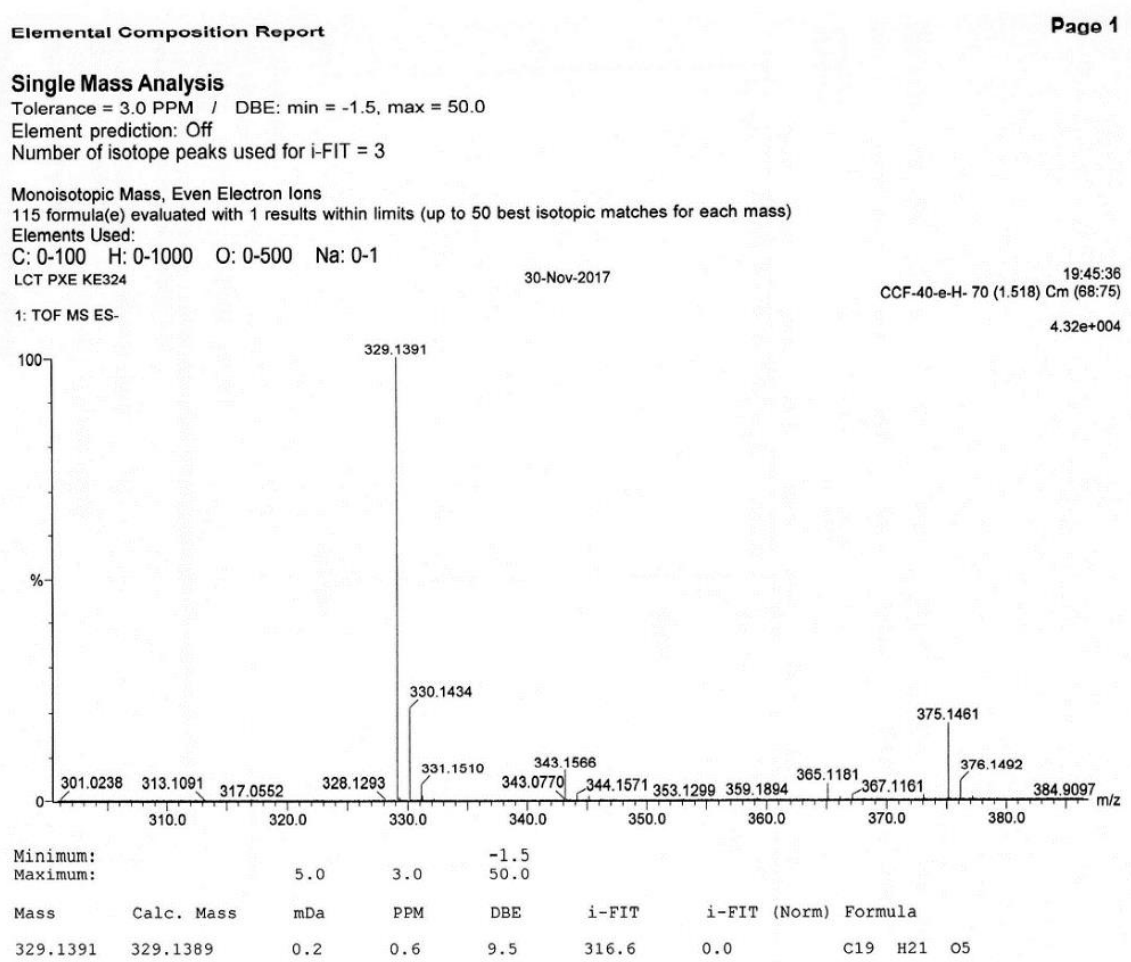


Figure S194. IR spectrum of fortalpinoid Q (17).

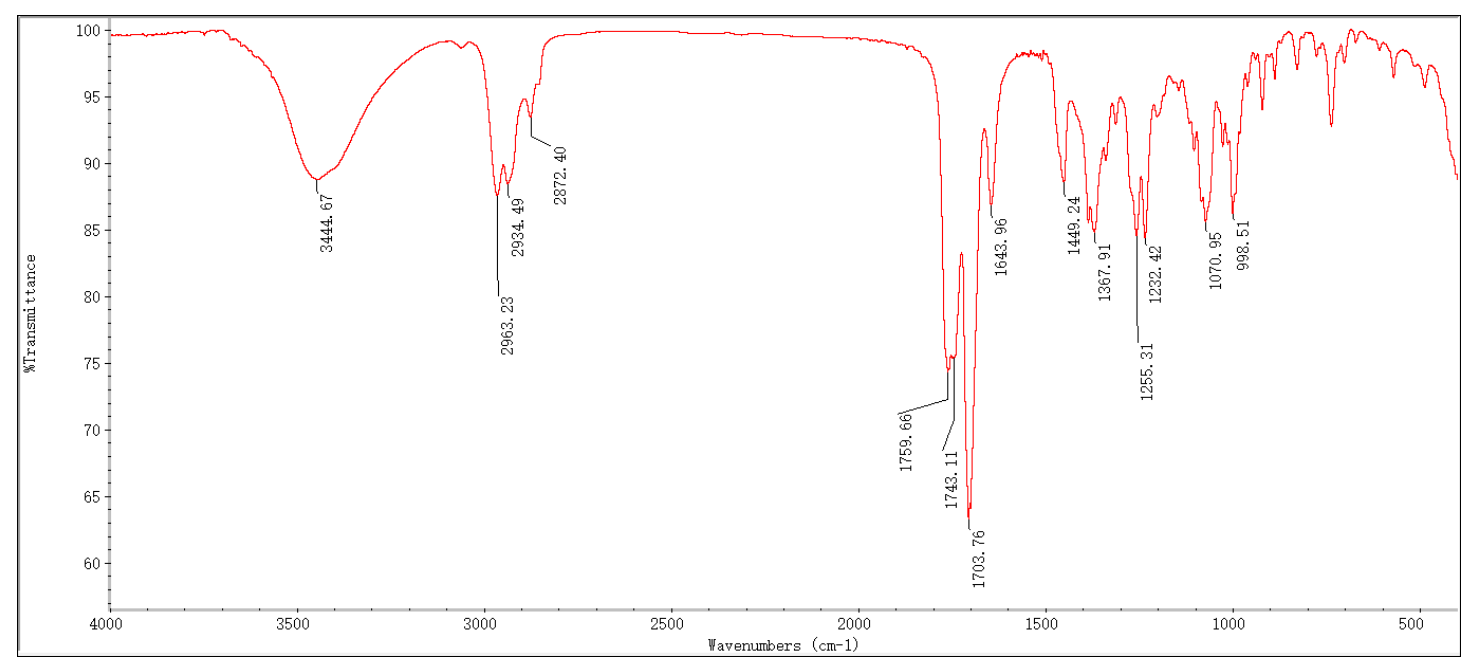

